\title{
Control of primate prehension movements in time and space
}

\author{
DisSERTATION \\ FOR THE AWARD OF THE DEGREE \\ "Doctor rerum naturalium" \\ OF the Georg-August-Universität Göttingen \\ Within the doctoral Program "Sensory And Motor Processing" \\ of the Georg-August University School of Science (GAUSS)
}

\author{
submitted by \\ Reinhard Stefan Greulich \\ from Halle (Saale), Germany
}

Göttingen 2021 
Thesis Committee

Supervisor/1st Referee

Prof. Dr. Hansjörg Scherberger

Neurobiology

Deutsches Primatenzentrum

2nd Referee

Dr. Igor Kagan

Cognitive Neurosciences Laboratory, DAG

Deutsches Primatenzentrum

Prof. Dr. Tim Gollisch

AG Sensory Processing in the Retina

University Medical Center Göttingen

\section{Members of the Examination Board}

Dr. Caspar M. Schwiedrzik

Neural Circuits and Cognition

European Neuroscience Institute Göttingen

Prof. Dr. Susann Boretius

Functional Imaging

Deutsches Primatenzentrum

Prof. Dr. Melanie Wilke

Cognitive Neurology

University Medical Center Göttingen

Date of oral examination: $19^{\text {th }}$ of March 2021 
Clarity of thought is a shining point in a vast expanse of unrelieved darkness. Genius is not so much a light as it is a constant awareness of the surrounding gloom, and its typical cowardice is to bathe in its own glow and avoid, as much as possible, looking out beyond its boundary. from "His Master's Voice" by Stanisław Lem 


\section{Acknowledgments}

Sir Isaac Newton clarified his position in science by coining the famous quote "If I have seen further it is by standing on the shoulders of Giants." This page is dedicated to the humans and non-humans who helped me climb those shoulders. I wish to extend my sincere thanks to:

My former supervisors Dr. Franziska Schneider, Prof. Dr. Peter Hegemann, Prof. Dr. John-Dylan Haynes, and Prof. Dr. Graham Lieschke for their teaching and shaping of my scientific career.

My current supervisor Prof. Dr. Hansjörg Scherberger for inviting me into his lab, his ongoing support and making this thesis possible.

Dr. Igor Kagan for the insights into macaque fMRI and agreeing to be on my thesis committee. Prof. Dr. Tim Gollisch for also joining my thesis committee.

My parents and family for their ongoing support.

My grandfather for lighting my interest in science and mathematics. I wish you could have celebrated this day with us.

Ben, Johnathan, and Swathi for the fruitful discussions and advice.

Natalie and Sabine for their invaluable help with animal training.

Mathias and Timo for their technical support.

And everyone else from the lab.

Dr. Thomas Christophel und Dr. Carsten Allefeld for answering all my questions regarding the cvMANOVA.

The Kagan and Boretious group for their help with the MRI scanning.

Prof. Dr. Alfred Benedikt Brendel for being a good friend, collaborator and sensei.

All my human subjects.

Moe, Carl, and Millhouse for their past and ongoing sacrifices in the name of science.

My girlfriend Caro for her love and support.

All my friends who can't be mentioned individually, because it would turn this page into an extra chapter.

Hel and Fenrir for all the purrs and cuddles. 


\section{Table of Contents}

Acknowledgments V V

Zusammenfassung VIII

Abstract IX

Abbreviations $\quad$ X

1. General Introduction 1

1.1. Reach out to grasp fate . . . . . . . . . . . . . 1

1.2. Control of prehension actions in space. . . . . . . . . . . 2

1.2.1. Reaching in the dorso-medial stream . . . . . . . . 2

1.2.2. Grasping in the dorso-ventral stream . . . . . . . . . . 6

1.2.3. The controversy . . . . . . . . . . . . . . 7

1.2.4. Prehension in humans . . . . . . . . . . . . 7

1.3. Control of prehension actions in time . . . . . . . . . 10

1.3.1. Why the watched kettle never boils . . . . . . . . . 10

1.3.2. Getting a grasp on time . . . . . . . . . . . . . . 12

1.4. A short history of functional magnetic resonance imaging . . . . . 13

1.4.1. The blood oxygen level dependent|signal . . . . . . . . . . 14

1.4.2. History and current developments regarding /multi voxel pattern analysis $\ldots \ldots \ldots \ldots \ldots \ldots$

1.5. Aim of this thesis $\ldots \ldots \ldots \ldots$

2. Original Projects 20

2.1. Project I: Shared functional connectivity between the dorso-medial and dorso-ventral streams in macaques. . . . . . . . . . . . . 20

2.1.1. General Discussion . . . . . . . . . . . . . . . . . . 20

2.2. Project II: PriMa: A low-cost, modular, open hardware, and 3Dprinted $|\mathrm{fMR}|$ manipulandum . . . . . . . . . . . . . . . . 40

2.2.1. General Discussion . . . . . . . . . . . . . . . . . . 40 
2.3. Project III: Difference in motor initiation for a predictable or unpredictable go-cue in humans and $\mid \mathrm{NHP} s$ utilizing a $|\mathrm{fMRI}|$ grasp paradigm and lcvMANOVA . . . . . . . . . . . . . . . . . . . 63

2.3.1. General Discussion . . . . . . . . . . . . . . . . . . . . 63

2.3.2. Introduction . . . . . . . . . . . . . . . . . . . . 65

2.3.3. Materials \& Methods . . . . . . . . . . . . . . . . . . 66

2.3.4. Results . . . . . . . . . . . . . . . . . . 75

2.3.5. Discussion . . . . . . . . . . . . . . . . . . . . . . . . . . 90

3. General Discussion 103

3.1. The reach and grasp networks revisited . . . . . . . . . . . . . . 103

3.2. Initiation of hand actions . . . . . . . . . . . . . . . . . 105

3.3. Conclusion . . . . . . . . . . . . . . . . . . . . . 107

3.4. Outlook . . . . . . . . . . . . . . . . . . . . . . 107

\begin{tabular}{lr}
\hline 4. Bibliography & 110
\end{tabular}

\begin{tabular}{ll}
\hline A. Additional Publications & 161
\end{tabular}

A.1. Brendel, A., Greulich, R.S., Niederman, F., Trang, S., 2020. Towards a Greater Diversity of Replication Studies. AIS Transactions on Replication Research 6. . . . . . . . . . . . . . . . . . . . . . . . 161

\begin{tabular}{ll}
\hline B. Mass univariate analysis of project III & 177
\end{tabular}

B.1. Materials \& Methods . . . . . . . . . . . . . . . . . . . . . . . . . 177

B.2. Results . . . . . . . . . . . . . . . . . . . . . . . . . . . 178

B.2.1. Mean effect . . . . . . . . . . . . . . . . . . . . . 178

$\begin{array}{ll}\text { C. Supplemental Material for project II } & 185\end{array}$

\begin{tabular}{lr}
\hline D. Additional Resources & 189
\end{tabular}

\begin{tabular}{ll}
\hline E. Selbstständigkeitserklärung & 190
\end{tabular} 


\section{Zusammenfassung}

Greifbewegungen in Primaten erlauben eine diverse Interaktion mit der Umwelt. Sie können in zwei separate Vorgänge getrennt werden: den Transport des Armes zum Objekt, genannt Ausstrecken (reach) und dem Zufassen und Manipulieren des Objektes, genannt Zugreifen (grasp). Es ist ungeklärt ob beide Vorgänge zusammen oder getrennt von zwei Netzwerken im Gehirn gesteuert werden, dem dorso-medialen und dorso-ventralen Netzwerk.

Diese Doktorarbeit präsentiert zwei Experimente welche diese Fragestellung erforscht. Im ersten Experiment wird die funktionale Verbindung zwischen beiden Netzwerken in Makaken unter Narkose mit funktionaler Magnetresonanztomographie untersucht. Das zweite Experiment ergründet die Encodierung von beiden Prozessen in Menschen mit funktionaler Magnetresonanztomographie und Multi Voxel Musteranalyse mit cvMANOVA.

Um das zweite Experiment zu ermöglichen, wurde ein 3D gedrucktes MRI-kompatibles modulares Manipulandum konstruiert. Die Flexibilität dieses Manipulandum ermöglicht weitere Erforschung von Arm und Hand Handlungen in Menschen und Makaken.

Greifbewegungen müssen nicht nur räumlich, sondern auch zeitlich präzise sein. Um das Ziel einer Handlung zu erreichen, muss sie zum passenden Zeitpunkt eingeleitet werden. Das zweite Experiment untersucht diesen Prozess in Menschen, indem vorhersagbare mit überraschenden Go-Cues verglichen werden.

Die Ergebnisse unterstützen die Hypothese, dass reach und grasp von zwei getrennten Netzwerken gesteuert werden. Die funktionale Verbindung in Makaken zeigt eine klare Trennung zwischen beiden Netzwerken. In Menschen werden beide Prozesse in getrennten Gerhirnbereichen enkodiert und zeigen keine Überschneidung. Der Gyrus postcentralis enkodiert das Zugreifen und der Lobulus parietalis superior das Ausstrecken. Nur wenige Voxel im Sulcus postcentralis zeigen Interaktionseffekte.

Die Initiierung wird in der supplementär-motorischen Rinden enkodiert, in Übereinstimmung mit der existierenden Literatur über Zeitintervall Repräsentation. Weitere Cluster sind im prämotorischen und ipsilateralen primären motorischen Cortex zu finden. Eine Hypothese bezüglich der Funktion dieser Areale in der Initiation wird präsentiert. 


\section{Abstract}

Prehension in primates allows diverse interactions with the environment. It can be separated into two processes: the transport of the arm to the object, termed reach, and the getting hold and manipulation of the object, termed grasp. There is ongoing debate if and how both processes are controlled separately or together by two brain networks, called the dorso-medial and dorso-ventral streams.

In this thesis, two experiments examine this question in detail. The first utilizes macaque resting state functional magnetic resonance imaging and examines the functional connectivity of areas of both streams. The second examines the encoding of reach and grasp in humans with functional magnetic resonance imaging and multi voxel pattern analysis utilising cvMANOVA

To enable the second experiment, a 3D printed, MRI-compatible, modular manipulandum was designed. The flexibility of this manipulandum allows for further experimental examination of hand and arm actions in both humans and macaques.

Additionally, prehension requires not only control in space but also in time. For a motor action to be meaningful, it has to be initiated at the right time. The second experiment examines the question of how the brain manages this timely initiation in humans by comparing a predictable with an unpredictable go-cue.

The results provide further evidence for the two-stream hypothesis. Functional connectivity in lightly anesthetized macaques demonstrates a clear separation between both networks. In humans, we observe individual areas encoding either reach or grasp without overlap. The postcentral gyrus shows grasp encoding and the supraparietal lobule shows reach encoding. Only a few voxels in the postcentral sulcus show an interaction effect.

The initiation shows significant encoding in the supplementary motor area, confirming previous research into time interval representation in the brain. Additionally, we see widespread clusters in the premotor and ipsilateral primary motor cortex. A new hypothesis regarding motor initiation is formulated.

In conclusion, this thesis provides further support for the two-stream hypothesis, insights into the initiation of prehension movements in humans, and presents a novel manipulandum to investigate prehension actions. 


\section{Abbreviations}

AAL automated anatomical labelling atlas third version.

ACC anterior cingulate cortex.

ADHD attention deficit hyperactivity disorder.

AIP anterior intraparietal area.

aIPS human anterior intraparietal sulcus.

ANOVA analysis of variance.

BA brodmann area.

BG basal ganglia.

BOLD blood oxygen level dependent.

BP Bereitschaftspotenzial.

CBF cerebral blood flow.

CBV cerebral blood volume.

CNV contingent negative variation.

$\mathrm{CRMO}_{2} \quad$ cerebral metabolic rate of oxygen.

cVMANOVA cross-validated multivariate analysis of variance.

d.f. degrees of freedom.

EEG electroencephalography.

EPI echo planar imaging.

FEF frontal eye field.

FIR finite impulse response.

fMRI functional magnetic resonance imaging.

FoV field of view.

FWE family wise error.

FWHM full width at half maximum. 
GLM general linear model.

HRF hemodynamic response function.

ICBM International Consortium of Brain Mapping.

IPS intraparietal sulcus.

ITI inter trial interval.

LED light emitting diode.

M1 primary motor cortex.

$M 1_{\text {hand }} \quad$ hand area of the primary motor cortex.

MDP medial dorsal parietal cortex.

Mean Sq. Mean of squares.

MI motor imagery.

MIP medial intraparietal area.

MNI Montreal Neurological Institute.

MP-RAGE magnetisation-prepared rapid gradient-echo.

MRI magnetic resonance imaging.

MT movement time.

MVPA multi voxel pattern analysis.

NHP non human primates.

PD Parkinson's disease.

PMd dorsal premotor cortex.

PMv ventral premotor cortex.

POS parieto-occipital sulcus.

PrCO precentral opercular area.

PRR parietal reach region.

RT reaction time.

SI primary somatosensory area.

SII secondary somatosensory area. 
SMA supplementary motor area.

SNR signal-to-noise ratio.

SPL superior parietal lobule.

SPOC superior parieto-occipital cortex.

std standard deviation.

Sum Sq. sum of squares.

TE echo time.

TMS transcranial magnetic stimulation.

TR repetition time.

V6 visual area 6.

V6A visuomotor subdivision of $V 6$ 


\section{General Introduction}

One of the defining features of primates is the opposable thumb (for review see Diogo et al., 2012). Allowing fine manipulation using individual fingers and the thumb, while other species like rodents are only able to grasp objects by wrapping the whole forelimb around it in a sort of palm grasp (for a comparison of reach to grasp behavior over a range of species see Whishaw and Karl, 2014). Along with the ability to oppose their thumbs, primates needed to develop the cognitive ability to control those actions of the hand and arm. This thesis explores the spatial and temporal control of those reach and grasp actions in humans (homo sapiens) and the non human primates (NHP) species macaca mulatta.

\subsection{Reach out to grasp fate}

The use of the forelimbs for more than locomotion is at least as old as the superclass of tetrapods itself. The ability for skilled forelimb movements (defined as reach and grasp for objects and the manipulation thereof with the fingers) is present in all mammalian species, except for monotremes (Iwaniuk and Whishaw, 2000). In primates, the arboreal life and need to obtain food from terminal tree branches gave evolutionary pressure to further develop the manipulation abilities of the limbs (for review see Sustaita et al., 2013), leading to the development of the ability to grasp objects between forefinger and thumb (precision grasp; Napier, 1956) in or before the infraorder of anthropoids (MacNeilage, 1990, Pouydebat et al., 2008). From all members of this infraorder, humans have developed the highest manipulative potential of the hand (Liu et al., 2016).

Prehension movements in primates and humans can be separated into two distinct processes, the transport of the hand towards the object, termed the reach, and the closing of the fingers around the object, termed the grasp (Jeannerod, 1999). Karl and Whishaw (2013) argue, that both have separate evolutionary origins with the reach descending from stepping behaviour and the grasp from feeding (also see Whishaw and Karl, 2014, for further elaboration of their argument). This separation 
of evolutionary origin is a possible explanation for the separation on a neurological level between both processes, which has been termed the two stream hypothesis of visiomotor processing (Jeannerod, 1999).

\subsection{Control of prehension actions in space}

The two stream hypothesis separates the control of prehension into a dorso-ventral stream, controlling grasp and a dorso-medial controlling reach (Jeannerod, 1981. 1999; see figure 1.1 on the facing page). The main support for thesis came from anatomical tracer studies and physiological recordings in macaques. Both streams lead from the visual cortex to the primary motor cortex, transforming visual information into motor actions (also called the perception-action model Goodale, 2011).

The next two sections give a short introduction into the two separated streams and the evidence supporting them in NHP. The following section gives an overview of the recent evidence against the two-stream hypothesis, while the final section shows the current state of research regarding prehension movements in humans.

A note on terminology: This thesis uses the terms dorso-medial and dorso-ventral for both branches of the dorsal stream. This terminology reflects the flow of information in the brain and emphasizes the inclusion of areas in the medial wall in the dorso-medial stream. Both streams were initially termed dorso-dorsal and ventraldorsal by Rizzolatti and Matelli (2003), following a posterior-anterior anatomical nomenclature. However, the terms are interchangeable.

\subsubsection{Reaching in the dorso-medial stream}

Jeannerod (1999) proposed that the dorso-medial stream goes from early visual cortex over area V6/V6A either directly or via two branches to dorsal premotor cortex (PMd). Galletti et al. (1993) showed neurons encoding the spatial location of objects independent from the animal's gaze in area V6, an area that is both directly connected to PMd and via medial intraparietal area (MIP) in the superior bank of the intraparietal sulcus (IPS) (Johnson et al., 1993, Lewis and Van Essen, 1994; Tanne et al., 1995; Tanné et al., 1995; Marconi et al., 2001; Bakola et al., 2017). Further electrophysiological recordings in $\mathrm{V6 \textrm {A }}$ reported reach encoding in multiple reference frames (i.e. relative to eye, hand or body) and stages of planning and execution (Galletti et al., 1997. Snyder et al., 1997, 1998, Galletti et al., 1999, Battaglia-Mayer et al., 2000: Fattori et al., 2001, 2005, Marzocchi et al., 2008, Bosco et al., 2010. Chang and Snyder, 2012; Breveglieri et al., 2013; Hadjidimitrakis et al., 2014; Bosco 

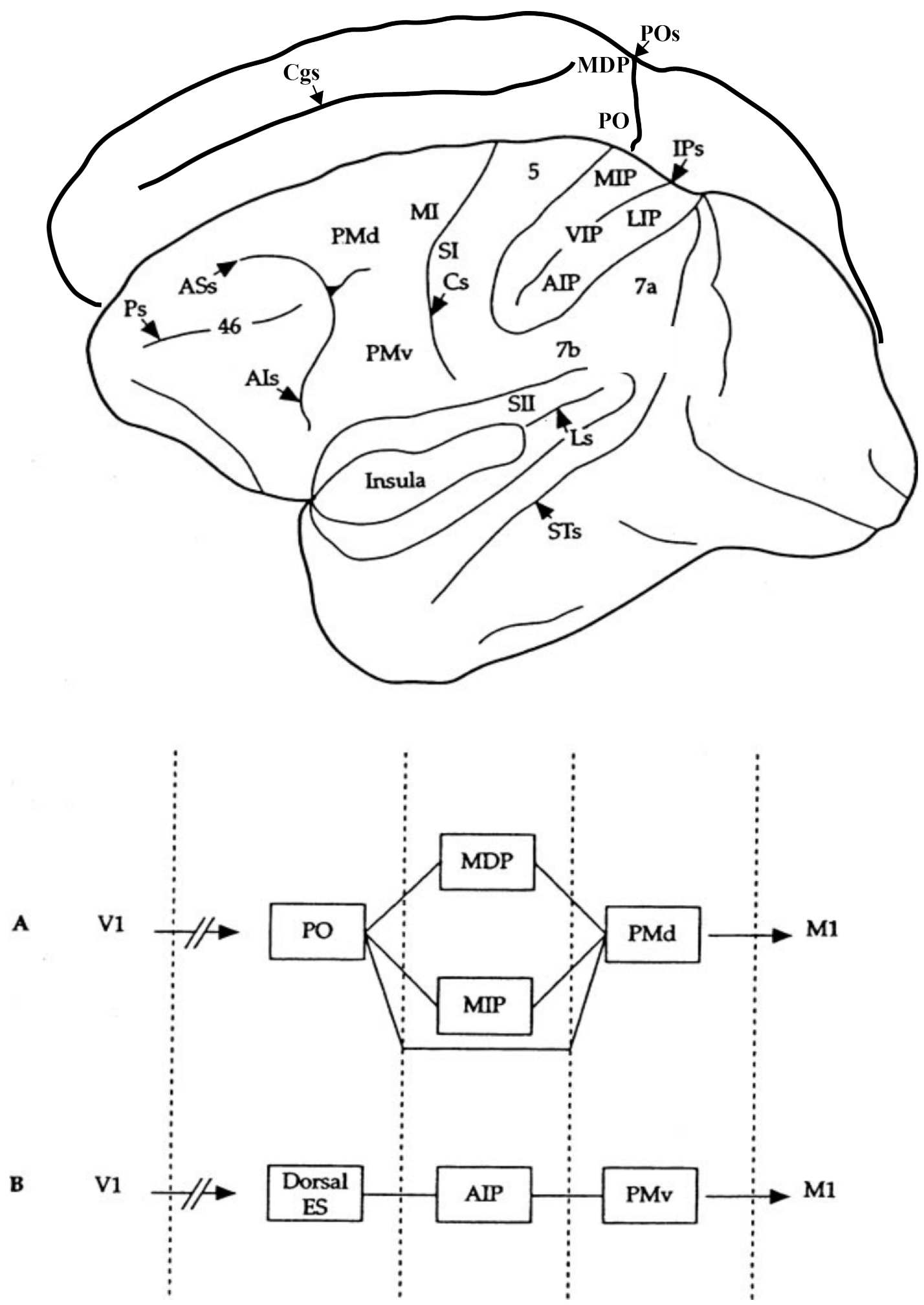

Figure 1.1.: The two streams in the macaque cortex as originally published in Jeannerod (1999) Top panel: Anatomical structures and areas of the macaque cortex as used by and modified from Jeannerod (1999). Bottom panel: The dorso-medial stream (A) and dorso-ventral stream (B), as proposed by and taken from Jeannerod (1999). Continued on next page. 
Figure 1.1.: Continued from previous page. The two streams in the macaque cortex as originally published in Jeannerod (1999). Abbreviations: AIP anterior intraparietal area Als, inferior ramus of the arcuate sulcus; ASs, superior ramus of the arcuate sulcus; Cgs, Cingulate sulcus; Cs, central sulcus; ES, extocalcarine sulcus; IPs, intraparietal sulcus; LIP, lateral intraparietal area; Ls, lateral sulcus; MI, primary motor cortex. MDP medial dorsal parietal cortex; MIP medial intraparietal area PO, parietal occipital area; POs, parieto-occipital sulcus; Ps, principal sulcus; SI, primary sensory cortex; SII, secondary somatosensory area; STs, superior temporal sulcus; V1, primary visual cortex; VIP, ventral intraparietal area. This figure is provided to present the historical origins of the two stream hypothesis and this thesis uses partially differing abbreviations and nomenclature. For example, Jeannerod (1999) uses the term PO in this illustration. It was later noted that this area is not consistently defined across the literature and the more precise terms of visual area 6 (V6) and visuomotor subdivision of V6 (V6A) should be used (Galletti et al., 2005), a recommendation this thesis follows.

et al., 2015, Santandrea et al., 2018: Hadjidimitrakis et al., 2020). This earned area $\mathrm{V6A}$ in conjunction with the bordering MIP the term parietal reach region (PRR) (Snyder et al., 1997, 1998).

Mountcastle et al. (1975) were the first to record reach encoding cells in MIP Further research showed that MIP encodes the target of a reach both in eye and body entered coordinates (Kalaska et al., 1983 Lacquaniti et al., 1995; Buneo et al., 2002) and shows cells encoding motor plans for both reach and saccades (Snyder et al., 1997). While inactivation of MIP produces misreaches in the visual periphery (Hwang et al., 2012), the coordinating role of MIP between eye and hand in reaches is further strengthened by the observation that reach neurons in MIP react stronger to reaches in light than in darkness (Colby and Duhamel, 1991). MIP is both directly connected to V6A and the arm area of PMd (Johnson et al., 1993 Johnson et al., 1996; Battaglia-Mayer et al., 2000). It is therefore postulated that MIP is involved in the transfer from coordinate systems from eye enteric input to body enteric output (Cohen and Andersen, 2002). Another proposed function of MIP is the online control of the executed reach (Desmurget and Grafton, 2000, Kalaska et al., 2003 Grefkes and Fink, 2005), for which it receives input from subcortical areas and the cerebellum, transporting efference copies of motor commands, and vestibular and proprioceptive input (Prevosto et al., 2010, Ugolini et al., 2019). Further support for the online control hypothesis is the encoding of reach targets in both visual and proprioceptive relative encoding (McGuire and Sabes, 2011). 
The area termed medial dorsal parietal cortex (MDP) as used by Jeannerod (1999) is identical to areas PGm (also called $7 \mathrm{~m}$ in some publications) and PEc (compare M. F. S. Rushworth et al. 1997 and Lewis and Essen 2000 with Bakola et al. 2010 and Passarelli et al. 2018). Since both areas are directly adjacent, a clear identification of the recording area is often questionable and the identification of $P G m \backslash 7 m$ is still disputed (Passarelli et al., 2018). The connectivity of PGm suggests a more visuospatial role, since it is connected to frontal eye field (FEF) and area 46 (Passarelli et al., 2018), while PEc is strongly connected to PMd (Marconi et al., 2001) and PRR (Bakola et al., 2010), implying a limb motor function. Therefore we will assume Jeannerod (1999) was considering PEc in their hypothesis (for an extensive review of the connectivity of both areas see Gamberini et al., 2020). PEc has been extensively studied for reach actions. The earliest observation in PEc was that this area combines manual and occulumotor activity (Ferraina et al., 1997). The main function is the encoding of the reach target in a hand reference frame with modulations of the eye gaze direction frames (Battaglia-Mayer et al., 2000, 2001; Ferraina et al., 2009. Bremner and Andersen, 2012. Sayegh et al., 2014; Hadjidimitrakis et al., 2015 Piserchia et al., 2017). In addition an encoding of reach targets and motor plans in mixed visual and proprioceptive encoding has been observed (Breveglieri et al., 2008 McGuire and Sabes, 2011).

PMd is the final area in the dorso-medial stream before both streams meet again in the primary motor cortex (M1). The separation of the premotor cortex into PMd and ventral premotor cortex (PMv) is based on the different connections both areas exhibit when considering the spinal (He et al., 1993), thalamic (Rouiller et al., 1999), cross hemispheric (Boussaoud et al., 2005) and intrahemispheric projections (Kurata, 1991; Johnson et al., 1993; Ghosh and Gattera, 1995: Tanné-Gariépy et al., 2002b). However, both areas are also separable by function. Kurata and Tanji (1986) observed neurons responding to passive manipulation of the distal forelimb in PMv and of passive manipulation of the proximal forelimb parts in PMd.

The first electrophysiolcal recordings in PMd were done by Kubota and Hamada (1978) showing neural activity during a simple visual tracking task, where the macaque indicated the position of a stimulus by rotating a cylinder. Weinrich and Wise (1982) reported neural activity during a reach task, leading to a plethora of other studies investigating neural activity during reach in PMd (for example Caminiti et al., 1991; Johnson et al., 1993; Crammond and Kalaska, 1996, Johnson et al., 1996. Crammond and Kalaska, 2000, Messier and Kalaska, 2000, Cisek and Kalaska, 2002 Hoshi and Tanji, 2002, Churchland et al., 2006a b; Batista et al., 2007, Gail et al., 2009. Boudrias et al., 2010, Song and McPeek, 2010). The PMd encodes the speed 
(Churchland et al., 2006a), direction (Messier and Kalaska, 2000. Cisek and Kalaska, 2002), distance (Messier and Kalaska, 2000) and arm orientation (Scott et al., 1997) in multiple reference frames (Batista et al., 2007). The hypothesized function of the PMd in reach processing is to provide a rule-based action representation (Gail et al., 2009) and cognitive control (Mirabella et al., 2011) of upper limb movements.

\subsubsection{Grasping in the dorso-ventral stream}

Jeannerod et al. (1995) proposes the grasp network consists of only two areas, anterior intraparietal area (AIP) and the subsection of $\mathrm{PMv}$ termed F5. The first observation of neural activity during general object manipulation in AIP was by Hyvärinen and Poranen (1974). More evidence for the function of AIP was gained by Haaxma and Kuypers (1975) who observed errors in grasping and fine finger control in macaques with ablations of the anterior bank of the intraparietal sulcus. Faugier-Grimaud et al. (1978) further refined those findings and located AIP as the source of the loss of grasp functions during ablations. Similarly, inactivation by muscimol injection leads to defects in preshaping during reach-to-grasp behaviour (Gallese et al., 1994). Neural recordings in AIP confirmed object shape, grasp (Taira et al., 1990 Sakata et al., 1995: Murata et al., 1996 Murata et al., 2000; Baumann et al., 2009) and grasp force selective neurons (Intveld et al., 2018). AIP is a primary target for the decoding of grasping actions for neural prosthetics (Schaffelhofer et al., 2015) and is strongly connected to secondary somatosensory area (SIII) and F5 (Luppino et al., 1999; Borra et al., 2008).

F5 is strongly connected to the hand and arm area of M1 (Matsumura and Kubota, 1979). Rizzolatti et al. (1981) recorded neurons responding to stimulation of the hand and mouth area as well as neurons responding to passive joint manipulation of the forearm and hand. Further electrophysiological studies showed strong grasp encoding neurons in F5 (Rizzolatti et al., 1988; Rizzolatti and Gentilucci, 1988; Di Pellegrino et al., 1992; Raos et al., 2006). The current understanding of the function of F5 is to encode the grasp posture (Raos et al., 2006; Fluet et al., 2010) and force of the upcoming grasp (Intveld et al., 2018). To achieve this, F5 transforms the object feature input from AIP into a posture encoding (Schaffelhofer and Scherberger, 2016). F5 neurons also show response to observation of hand actions, opening up the research into so-termed mirror neurons (Di Pellegrino et al., 1992; Gallese et al., 1996). Those neurons, and therefore the area F5 itself, play a role in social cognition and learning regarding grasp actions (for review see Heyes, 2010; Cook et al., 2014). 


\subsubsection{The controversy}

The hypothesis of Jeannerod (1999) has recently come under criticism. Neural activity has been observed correlating with reach processing in the dorso-ventral stream and grasp processing in the dorso-medial stream. This calls the separation between both streams in to question.

The first evidence against a separation of both streams was found in the premotorcortex. Kakei et al. (2001) showed neurons encoding the direction of a movement in PMv a condition which is considered to be a part of the reach process (Caminiti et al., 1991). This was followed by the findings of Stark et al. (2007), showing that reach and grasp in the premotor cortex are encoded independent of the location of the recording. Finally, Lehmann and Scherberger (2013) showed reach encoding neurons in F5 and AIP, while Nelissen et al. (2018) could decode the used grasp from $\mathrm{F} 2$, a subsection of $\mathrm{PMd}$, and $\mathrm{V} 6 \mathrm{~A}$ in a functional magnetic resonance imaging (fMRI) experiment employing multi voxel pattern analysis (MVPA).

The strongest evidence against a separation exists for area V6A. Galletti et al. (2003) were the first to report grasping neurons in this area of the dorso-medial stream. Those findings were successfully replicated and further refined in subsequent studies (Fattori et al., 2004, 2010, Gamberini et al., 2011, Breveglieri et al., 2016. Filippini et al., 2017).

All those studies only show neural activity, but not a causal relationship for the respective movement. The lesion study of Battaglini et al. (2002) in V6A has been interpreted as producing not only a missreach, but also missgrasp (Galletti et al., 2003), providing so far the strongest evidence for the causal role of V6A for grasp processing.

\subsubsection{Prehension in humans}

The origins of research into human prehension control are in observations of lesions inducing a condition termed optic ataxia (Bálint, 1909). Patients suffering from optic ataxia exhibit a disability to reach for and grasp objects that is not caused by motor or visual impairments (Perenin and Vighetto, 1988). Early observation located the affected brain area for optic ataxia to the superior parietal lobule (SPL) (Ratcliff and Davies-Jones, 1972, Auerbach and Alexander, 1981; Ferro, 1984 Pierrot-Deseilligny et al., 1986, Wolpert et al., 1998, Astafiev et al., 2003, Glover, 2003). The location of the lesion focus causing optic ataxie were originally located to the human anterior intraparietal sulcus (aIPS) and superior occipital cortex (Karnath and Perenin, 2005). 
Blangero et al. (2009) combined fMRI and meta-analysis of previously published lesion studies and located the foci for optic ataxia to the parieto-occipital junction and medial IPS. Although most patients suffer from both a disturbance of the reach as well as the grasp process, if the lesion spares the alPS, the grasp process remains intact (Binkofski et al., 1998, Cavina-Pratesi et al., 2010b). This was further supported by the experimental disruption of alPS with transcranial magnetic stimulation (TMS) leading to grasp impairments (Tunik et al., 2005). The observation that optic ataxia in humans mirrors the behaviour of macaques with lesions to the PEc and V6A (Battaglia-Mayer and Caminiti, 2002), lead to the conclusion that the human aIPS is analogue to the macaque AIP (Binkofski et al., 1998, Grefkes et al., 2002), and the parieto-occipital sulcus (POS) to the macaque PRR (Battaglia-Mayer and Caminiti, 2002; Connolly et al., 2003). Pitzalis et al. (2013) located the human analogue of the macaque $\mathrm{V6A}$ to an area posterior to POS. The area around the POS and IPS in humans therefore closely mirrors the macaque cortex at the same location in its layout and function (for reviews see Culham and Valyear, 2006, Vesia and Crawford, 2012; Andersen et al., 2014; see figure 1.2 on the next page).

Prehension studies in humans centered around aIPS give a very similar picture to macaque AIP. With activity in the alPS correlating with grasp type, grasp planning, object orientation and graspability (Grefkes et al., 2002; Culham et al., 2003; Shikata et al., 2003: Frey et al., 2005; Shmuelof and Zohary, 2006; Begliomini et al., 2007b: Króliczak et al., 2007, Cavina-Pratesi et al., 2010a) and deactivation utilising TMS producing disturbances of the grasp or grasp planning (Glover, 2003. Tunik et al., 2005; Rice et al., 2006; Davare et al., 2010; Verhagen et al., 2012). Since tracer studies in humans are not possible on ethical grounds, research on the connectivity of the areas is limited to functional connectivity. In a very well designed study, Davare et al. (2010) observed the influence of TMS induced inactivation of alPS on human PMv and primary motor cortex (M1), which was also probed by TMS pulses, showing a facilitation of PMv and M1 by alPS during grasping.

Research of human reaching behaviour has mapped the human PRR, showing that the medial IPS and around the POS (sometimes called the superior parieto-occipital cortex (SPOC) ) exhibit reach related activity, general upper limb control, and whether objects are within reach and target-arm integration (Pellijeff et al., 2006: Levy et al., 2007; Tosoni et al., 2008; Beurze et al., 2009; Filimon et al., 2009; Gallivan et al., 2009; Hinkley et al., 2009; Vesia et al., 2010; Y. Chen et al., 2014; Cappadocia et al., 2017). With TMS applied over this locations showing various influences on reach (Vesia et al., 2006, G. Koch et al., 2008; Vesia et al., 2008, 2010; Striemer et al., 2011, Vesia et al., 2013; Davare et al., 2015). G. Koch et al. (2008) showing 

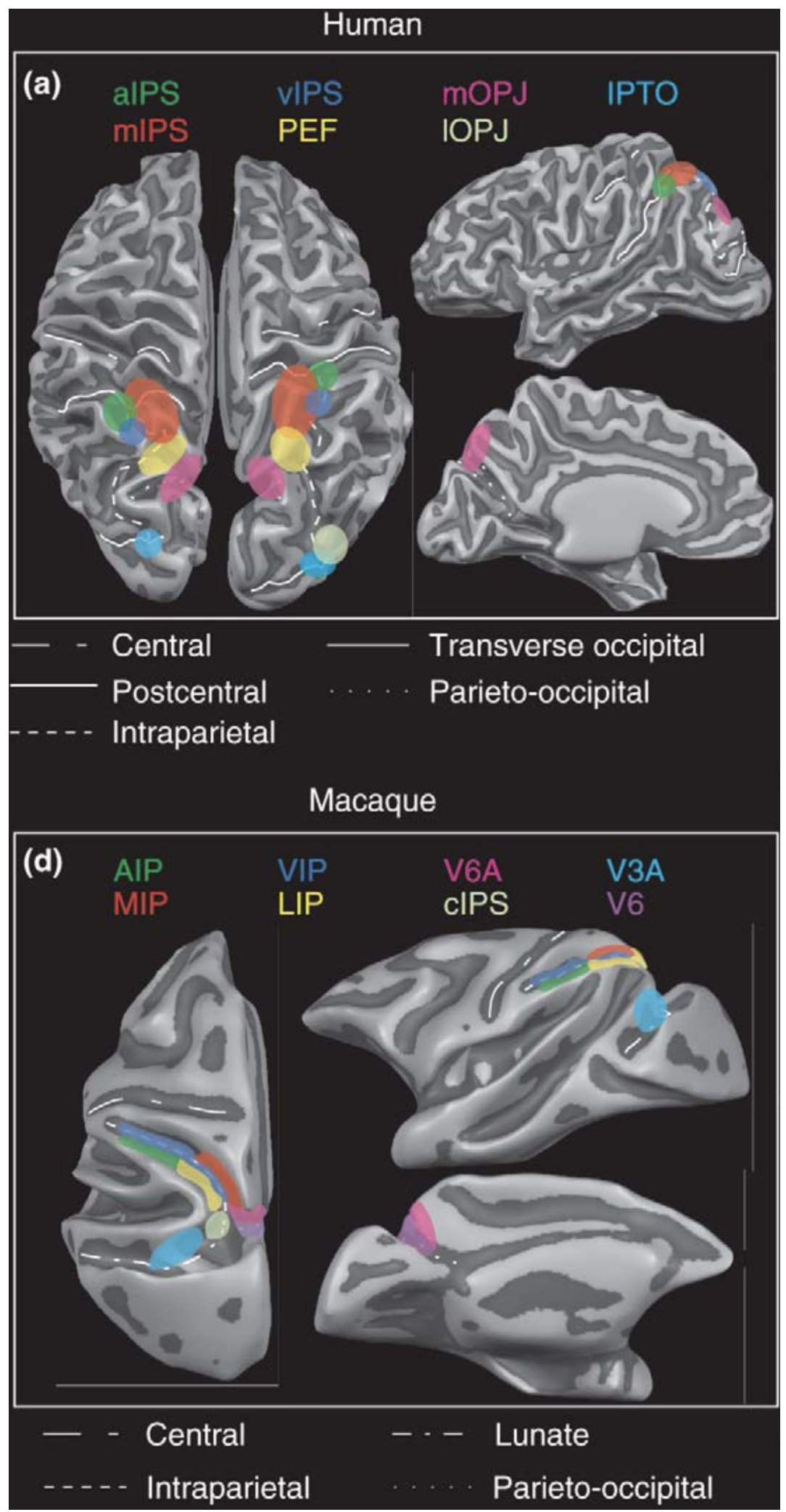

Figure 1.2.: Homolog areas in the parietal cortex between humans (top) and macaques (bottom). Abbreviations used: parietal eye field (PEF), occiptial parietal junction (OPJ), intraparietal and transverse occipital junction (IPTO), lateral intraparietal area (LIP), ventral intraparietal area (VIP). Illustration from Culham and Valyear (2006). 
facilitation of M1 by the human PRR during reach.

In an extensive fMRI study comparing reach-to-grasp and reach-to-lift with two different grasps, reach-to-touch and pointing, Cavina-Pratesi et al. (2018) separated reach and grasp into multiple subcomponents, precision of grasp, number of digits employed, arm transport, and required precision of locating the object. The authors then proceeded to locate those subcomponents to different brain areas: activation differences in the alPS correlated with grasp precision, alPS, PMd, and supplementary motor area (SMA) with object lift, PMv, M1 and primary somatosensory area (SI) with number of digits employed, and $\mathrm{PRR}$ with arm transport and lateral occipital cortex with location precision.

However, some studies in humans have also found results questioning the strict separation between the reach and grasp streams. In one of the earliest imaging studies into reach and grasping, Grafton et al. (1996) used positron emission tomography to investigate both processes. The authors observed only a difference in SII and subsequent $\mathrm{fMR}$ imaging studies found either no difference between both processes (Bono et al., 2015) or only grasping related activity in PMd (Grèzes et al., 2003).

\subsection{Control of prehension actions in time}

For a prehension action to achieve its goal it has not only to be precise in space, but also in time. The control of actions in time requires looking at two distinct processes. First, the way the brain tracks and calculates the passage of time. Second, the way the brain initiates motor actions at the point in time calculated using the first process. The following sections give a short introduction to both processes.

\subsubsection{Why the watched kettle never boils}

On the circuit level, Gibbon et al. (1984) presented a model for the computation of time in the brain (see figure 1.3 on the facing page). The first step in this circuit consists of a pacemaker producing a steady stream of pulses. Those pulses are gated by a switch presenting the attention to the passage of time, since a lack of attention is attributed with an subjective stretching of time (for reviews of the extensive research into this phenomena see Brown, 1997; Buhusi and Meck, 2009. Grondin, 2010). Burle and Casini (2001) showed that this phenomenon is caused by the lack of attention and not a different mechanism. Those gated pulses are then summed up in an integrator and compared to a reference value by a comparator. Neurons presenting ramping behavior (either up or down) consistent with integration 
of elapsed time are found over a great range of prefrontal areas during time estimation tasks (for review see Narayanan, 2016).

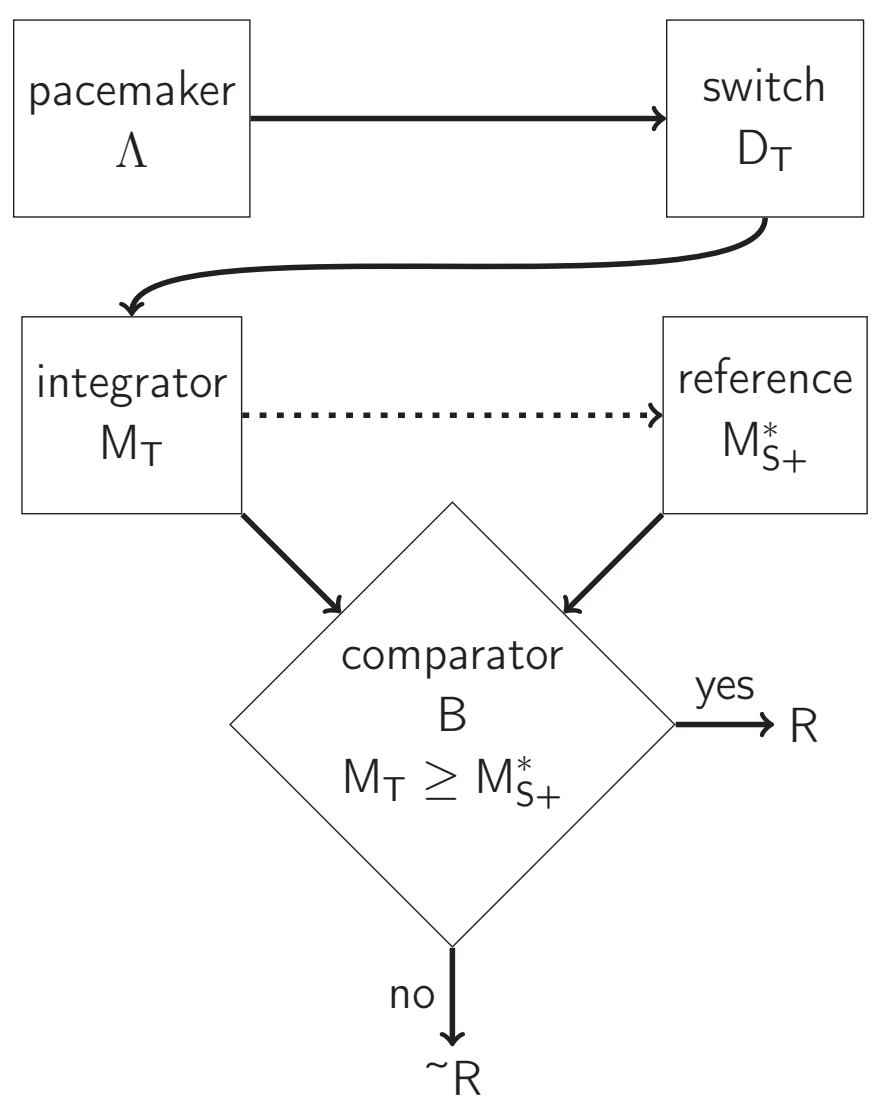

Figure 1.3.: Timing circuit as proposed by Gibbon et al. (1984). The pacemaker $(\Lambda)$ produces a pulse to be integrated in the working memory $\left(M_{T}\right)$ with attention acting as a switch $\left(D_{T}\right)$. A comparator $(B)$ checks the elapsed time $\left(M_{T}\right)$ against the reference value from memory $\left(M_{S_{+}}^{*}\right)$ and sets the behavioural outcome accordingly. After a time interval is successfully measured by the circuit, $\mathrm{M}_{\mathrm{T}}$ gets integrated in $\mathrm{M}_{\mathrm{S}+}^{*}$ to refine the time estimation process. Adapted from Gibbon et al. (1984)

While those ramping neurons are observed over a wide range of prefrontal locations, a meta-analysis of imaging studies in humans reveals two areas in the brain that show time dependent activity independent from the task conditions. Those two areas are the SMA and the right inferior frontal gyrus (Wiener et al., 2010), with the SMA showing distinct subdivisions regarding short or long estimated time intervals, sensory vs. sensorimotor, sequential vs. nonsequential and explicit vs. non explicit time calculations (Schwartze et al., 2012) . 


\subsubsection{Getting a grasp on time}

The presented timing circuit is used to find the point in time for an onset sensitive action. However, this still leaves the question open of how this action is actually initiated. The term initiation in the context of motor action is used to describe two different processes. The first context concerns the starting of the motor programming, while the later describes the execution of the motor program.

The research into the starting of the motor program has revolved around two characteristic brain potentials detectable by electroencephalography (EEG). The Bereitschaftspotenzial (BP) preceding self-initiated movements (Kornhuber and Deecke, 1965: for review see Shibasaki and Hallett, 2006) and the contingent negative variation (CNV) preceding externally cued movements (Walter et al., 1964). Since both signals precede the motor execution by an order of hundreds of milliseconds up to seconds (Walter et al., 1964 Kornhuber and Deecke, 1965), in the case of the BP even the conscious awareness of the decision to move (Libet et al., 1983), both are probably linked to the onset of the motor planning. The BP was located to the SMA (Shibasaki and Hallett, 2006; Lu et al., 2012) and the CNV to the PMd (Hultin et al., 1996; Lu et al., 2012). This leads to the conclusion that, although both being part of the motor network, the SMA is more involved in the initiation of voluntary movements and the PMd in externally cued movements (Okano and Tanji, 1987; Mushiake et al., 1991; Jenkins et al., 2000 but see Kurata and Wise, 1988 for a dissenting experiment).

Patients suffering from Parkinson's disease (PD) exhibit a difficulty to initiate movements called akinesia (Marsden, 1989). The cause of this akinesia is a disruption of the function of the basal ganglia (for review see Hauber, 1998 Obeso et al., 2000). The role of the basal ganglia (BG) in motor initiation appears to mimic the separation present in the cortical control (for review see Harrington and Jahanshahi, 2016), with an increased synchronicity between BG and the central cortical areas during self-initiated movements, and $\mathrm{BG}$ and the frontal cortex during external cued movements (Bichsel et al., 2018).

However, the separation between externally cued and self-initiated seems rather arbitrary (even without going into the question of whether there is free will or if we are just reacting to our environment in a quasi-chaotic manner, but see Haggard, 2008; for an examination of free will in neuroscience). Deecke (1996) uses the terminology of action for self-initiated and reaction for externally cued actions to separate the motor initiation from the question of how much self is in self-initiated. We will follow this terminology in the remainder of this thesis. Nature is not clearly 
separated in action and reaction. For example, in the martial art of Aikido, the aim is to be open to the actions of your opponent (reactive toward his actions), but to exploit said actions in an active manner (action) at the same time, reflecting the zen ideal of mushi (literally "empty mind", often interpreted as "acting without thinking"; Suzuki, 1959).

Although not discussed in the manuscript itself, project III (see section 2.3 on page 63) of this thesis is exploring the question whether there is an continuum regarding action and reaction. By employing different cues, one predictable and one unpredictable, it is tested if we can separate between pure reaction and reaction set onto a predictable moment in time. The latter could be interpreted as "acting at a predetermined point in time". If both conditions are purely reactive, we expect to see no difference in the $\mathrm{PMd}$ or SMA since both would employ the same neural network.

The second context for the term of motor initiation is the process to determine the time point for the execution of the prepared motor plan. Until 2016, the prevalent school of thought was that movements are executed as soon as the planning was complete (Donders, 1969, Rosenbaum, 1980, Erlhagen and Schöner, 2002, Cisek, 2006. Haith et al., 2016; Smeets et al., 2016). However, Haith et al. (2016) disproved that notion by showing, that the reaction time (RT) of an action includes additional processes, which can be omitted if the subject is forced to move at shorter then normal RT. This opened up the question if motor actions can be withheld, and if yes, how is the time point of the release of this withholding controlled. The first question, if motor actions can be withheld, was answered by Michaels et al. (2018), showing that the population dynamic in the motor and premotor areas diverges for movements for immediate execution and withheld movements for later execution. In this thesis, project III (see section 2.3 on page 63) provides an answer to the second question, how the release of the prepared movement is controlled in time.

\subsection{A short history of functional magnetic} resonance imaging

The basis of magnetic resonance imaging is found in magnetic resonance spectroscopy of protons in biological tissues. Utilising a strong magnetic field and radio pulses, those protons are brought into a synchronised spin state and emit specific radio waves when returning to the ground state. This effect can be separated into different processes, which can be measured by observing different relaxation times $\left(T_{1}, T_{2}\right.$, and $\left.\mathrm{T}_{2}^{*}\right)$. Those processes are influenced by the environment the proton experiences and 
strongly differ in different biological tissues (Odeblad and Lindstrom, 1955; Odeblad et al., 1956). The following section gives a short introduction to the basics of the blood oxygen level dependent (BOLD) signal and its relation to neuronal activity, as well as on the development of recent analysis methods. For a more extensive introduction into the physical and mathematical principles as well as the history of fMRI, the valued reader is directed to the excellent book of Huettel et al. (2014) "Functional Magnetic Resonance Imaging".

\subsubsection{The blood oxygen level dependent signal}

Early research into the magnetic properties of blood showed that deoxygenated haemoglobin shows paramagnetic properties (Pauling and Coryell, 1936), with following nuclear magnetic resonance spectroscopic examination showing an effect on the $\mathrm{T}_{2}^{*}$ relaxation time of protons (Brooks et al., 1975: Thulborn et al., 1982). The paramagnetic effect of deoxygenated haemoglobin leads to an disruption of the magnetic field and therefore a loss of signal in the $T_{2}^{*}$ contrast. Ogawa et al. (1990) were the first to utilise the magnetic differences between oxygenated and deoxygenated blood to image the brain.

Neural processing requires oxygen for various cell functions (e.g. reestablishing membrane potential, neurotransmitter release and uptake, and more). Therefore, an increase in neural load increases the local cerebral metabolic rate of oxygen (CRMO 2 . In theory, the difference between oxygenated and deoxygenated blood would allow the direct localisation of this increase in the brain. However, the brain shows a complex response cascade to local increase of $\mathrm{CRMO}_{2}$ rate that is triggered by the release of neurotransmitters (Faraci and Breese, 1993 Li and ladecola, 1994; Yang and ladecola, 1996) which leads to an increasing blood flow rate (CBF). The increased blood flow and flexibility of the vascular system leads to an increase of the total blood volume (CBV) and an oversupply of oxygenated blood (see figure 1.4 on the next page; Buxton et al., 1998, termed this effect the "balloon model"). The time delay between those processes leads to the characteristic form of the hemodynamic response function (HRF) with an dip from the initial depletion of oxygenated haemoglobin followed by a peak caused by the oversupply of oxygenated blood to the area (for reviews see Logothetis and Wandell, 2004; Buxton, 2012; Chen and Glover, 2015). 


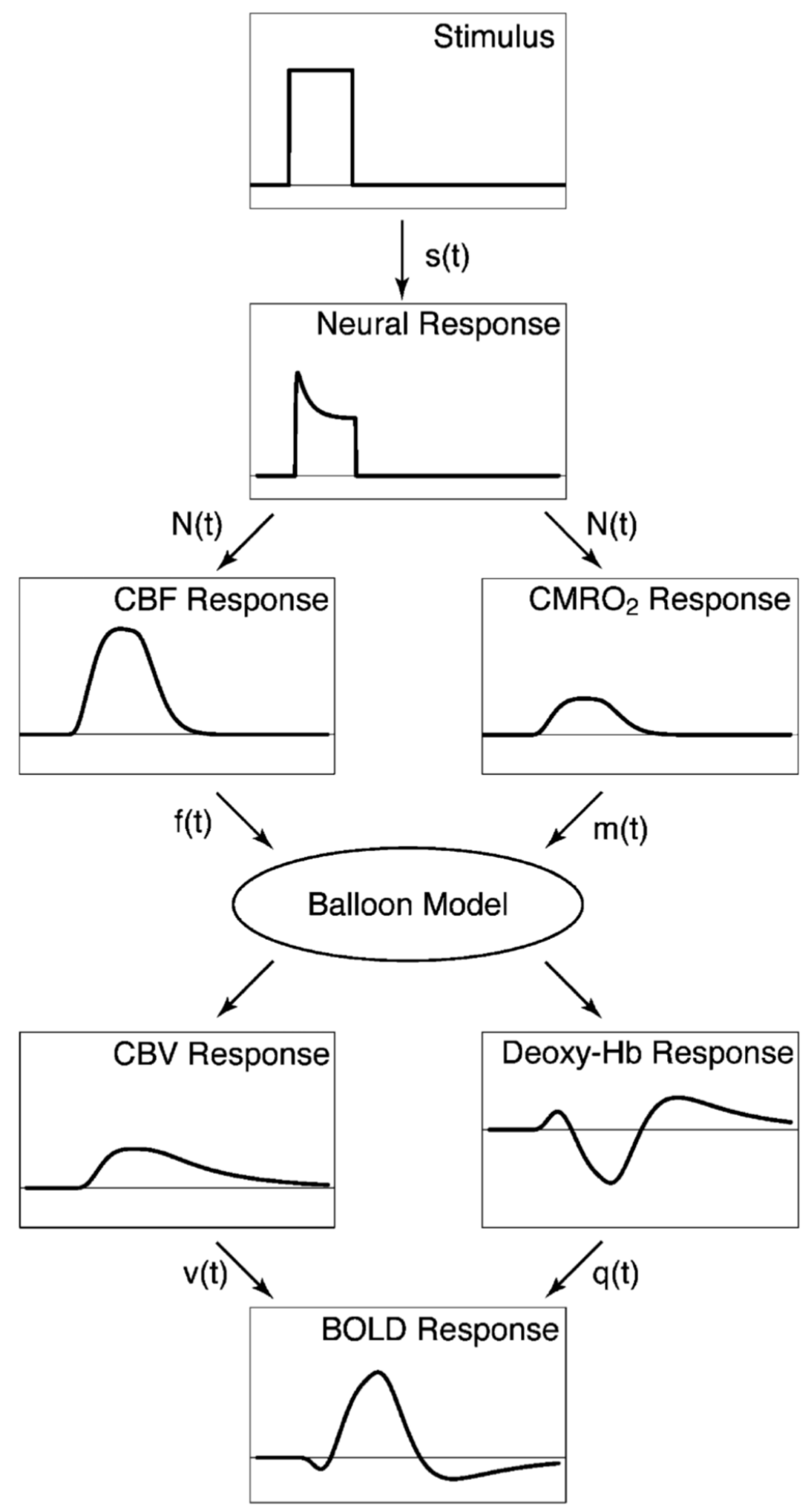

Figure 1.4.: The functions involved in the transformation from neural activity to the HRF. Neural activity is caused by the stimulus, which in turn increases the local $\mathrm{CBF}$ and $\mathrm{CRMO}_{2}$. With the flexibility of the vascular system increasing the CBV, while the $\mathrm{T}_{2}^{*}$ response of the blood is determined by the local conzentration of deoxygenated haemoglobin. All those factors lead to the characteristic HRF. Illustration from Buxton et al. (2004). 


\subsubsection{History and current developments regarding multi voxel pattern analysis}

Early $\mathrm{fMRI}$ experiments were analyzed by simply convolving the timeline of the experimental stimulation with the $\mathrm{HRF}$ and calculating the correlation of the signal time course from each voxel with said function (Bandettini et al., 1993). This correlation coefficient was then tested for significance. Friston et al. (1994b) extended this approach to allow the modelling of multiple experimental conditions in one analysis by utilising the general linear model (GLM). This analytical approach has been termed univariate analysis as each voxel is treated as a single variable and is independently analyzed (Friston et al., 1994a).

The univariate approach however suffers from a number of disadvantages. Since it considers each voxel independently, the interplay between areas of the brain is discarded (Kriegeskorte et al., 2006, Haxby, 2012; Chen and Glover, 2015). This is even exaggerated by the standard practice of including a smoothing step in the preprocessing, which increases statistical power for the univariate analysis, however also removes fine-grained information from the BOLD signal (Friston et al., 1994c: Kriegeskorte et al., 2006).

In the first study implementing MVPA, Haxby et al. (2001) disproved the previously held notion that the ventral occipital cortex areas only encode one set of objects individually. The experiment examined the patterns of BOLD activity in the fusiform face area and the parahipocampal place area during presentation of different stimuli sets (faces, houses, cats, and everyday objects), instead of asking which set of stimuli evoked the maximal bold response. The authors showed distinct patterns for each stimuli set in both areas, proving that both areas contain information about sets not evoking the maximal BOLD response. Since its inception, MVPA has been implemented in virtually every area of cognitive fMRI research (for reviews see Haynes and Rees 2006, K. A. Norman et al. 2006; Tong and Pratte 2011; Haxby 2012; Chen and Glover 2015, but also see Ritchie et al. 2019 for an analysis of the limitations of MVPA).

MVPA requires a definition of which voxels are included in the analysis in the experimental design. Early studies used predefined anatomical regions, functional predefined regions, or the whole brain (Haxby et al., 2001; Carlson et al., 2003. Cox and Savoy, 2003). However, those selection methods require a spatial hypothesis and do not allow for an unbiased estimation of the location of information in the brain. Kriegeskorte et al. (2006) solved this problem by introducing the searchlight approach. For each voxel in the brain, all voxels within a set radius are extracted, 
analyzed, and the results mapped back to the centre. When executed sequentially, this method resembles a searchlight, searching through the brain for areas encoding information. This leaves just the searchlight radius as the only a priori variable that has to be set for the analysis. It should be noted that searchlight analysis carries its own pitfalls regarding the interpretation of the results (Etzel et al., 2013). For example, a search light radius that is set to high produces large areas of significant results, since the information of a few individually informative voxels is mapped to the center of all searchlights containing said voxels. If the radius is set to low, the analysis risks missing results that are encoded in multiple low informative voxels (Etzel et al., 2013).

Many mathematical principles and algorithms have been used for pattern analysis in MVPA. Commonly used are so-called classifier algorithms such as linear discriminate analysis, support vector machines, and neural networks (Cox and Savoy, 2003 Hanson et al., 2004; O'Toole et al., 2005). Those classifiers all operate according to a similar principle. The data is separated into two sets, with the classifier being trained on one part and tested on the other. The resulting classification accuracy on the testing data set is then used as an indicator of the strength of pattern differences between the conditions (for an in depth tutorial see Pereira et al., 2009). This approach has been termed decoding of the brain and the used classifier as the decoder (Haynes and Rees, 2006). The advantage of decoding lies mainly in its simple implementation, toolboxes for support vector machines or neural networks are widespread, computationally optimized, and easy to use. However, the features of the classifier have to be carefully selected to avoid overfitting, which would lead to invalid results (Kamitani and Tong, 2005 K. A. Norman et al., 2006).

Another approach is to skip the classifier and describe the differences in patterns utilizing mathematical parameters such as Mahalanobis distance and pattern distinctness (see figure 1.5 on the following page; Kriegeskorte et al., 2006; Allefeld and Haynes, 2014). This method directly describes the BOLD signal differences of the analyzed set of voxels between the conditions, in other words the encoding of the conditions in the brain (for review see Sandberg et al., 2014). It circumvents the risk of selecting unsuitable classifier features and allows the modelling of interactions in multifactorial designs. Since classifiers can only separate the data into a fixed number of classes, interaction effects require multiple separate analyses and are difficult to interpret (for examples of multiclass decoding studies see Hausfeld et al., 2014, Lowe et al., 2016). The cross-validated multivariate analysis of variance (cvMANOVA) of Allefeld and Haynes (2014) circumvents those limitations entirely and allows for easily interpretable multifactorial MVPA 


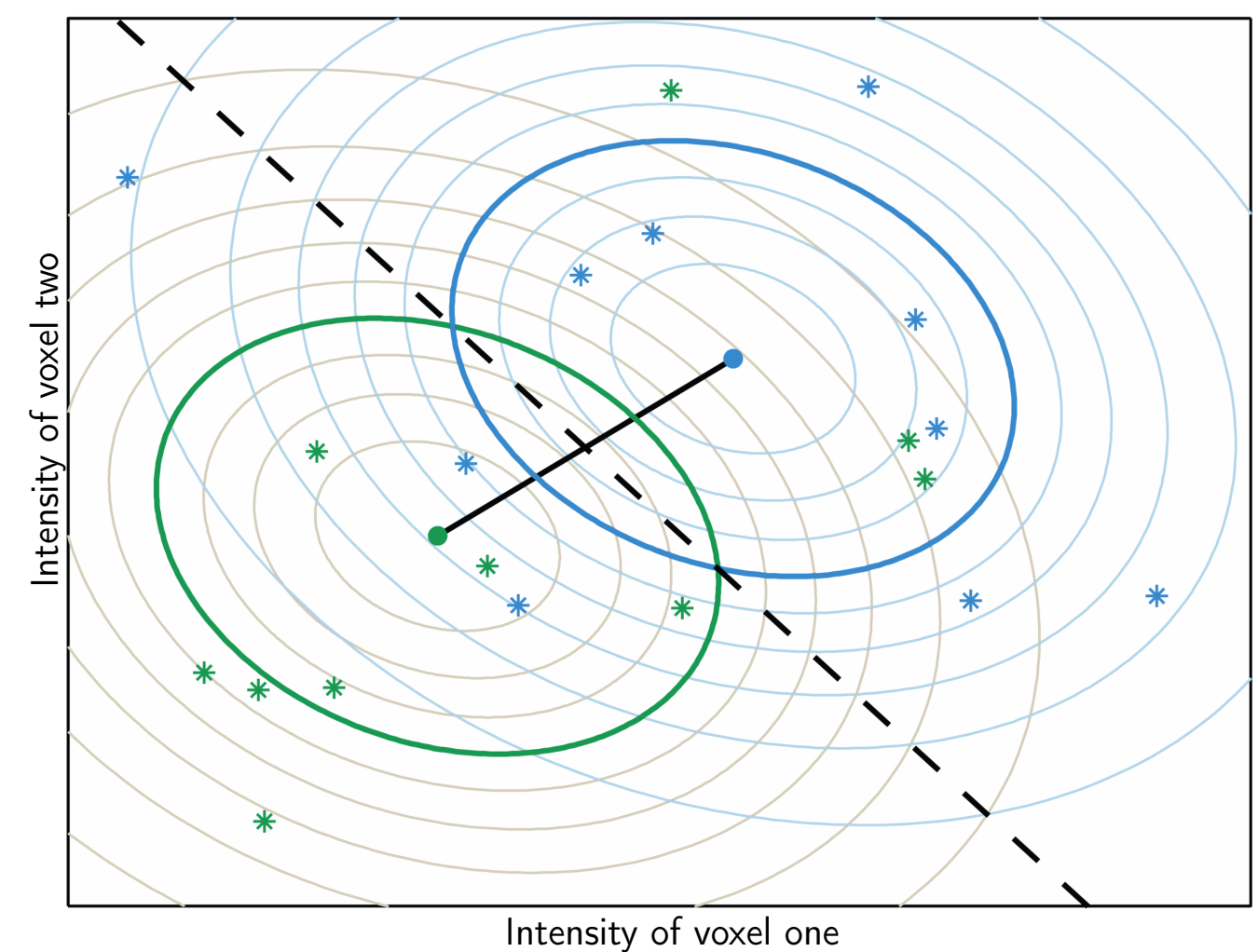

Figure 1.5.: Prinicples of MVPA demonstrated on a two voxel example. Each * demonstrates the intensity of the two voxels per recorded condition, with the conditions coded by colour. The solid dot marks the mean and the coloured ellipse the standard deviation of each distribution of the two conditions. In decoding, the classifier tries to find the best separation boundary between both conditions (dashed line) while an encoding approach aims to measure the distance between both distributions (solid line). Modified from Allefeld and Haynes (2014)

\subsection{Aim of this thesis}

To perform a successful prehension action, it has to be precise in space as well as in time. Precision in space separates into two demands. The first is to precisely reach the point in space where the object to be grasped is located. Secondly, the hand has to adapt to the shape of the object and precisely close around it to complete the grasp. The debate regarding how those parts of prehension movements are controlled is still ongoing as demonstrated in section 1.2 on page 2. Even the question if both are controlled by one or two different processing streams in the primate brain remains open. This thesis aims to further investigate this control.

The separation between the reach and grasp process is mainly informed by anatomical connections in the macaque brain. Electrophysiological recordings have provided a mixed picture. However, the presence of neural activity does not have to be causal 
to the condition it represents (for review see Vaidya et al., 2019). Likewise, anatomical connectivity only shows direct monosynaptical links, while functional connectivity also detects connections that are facilitated by indirect connections (M. A. Koch et al., 2002).

In project I (see section 2.1 on the following page), this thesis investigates the functional connectivity of areas of both streams in macaques. The separation is tested by clustering algorithms. If the involved areas do not separate on a functional level into two networks, then the two stream hypothesis proposed by Jeannerod (1981) is false and the separation between both purely anatomical.

Further insight into the control of prehension actions in humans and NHP can be gained from experiments with behaving subjects. However, the investigation of reach and grasp faces a range of difficulties in $f M R I$ research. The MRI environment puts a number of demands on the experimental setup regarding safety and signal disruption (Dempsey and Condon, 2001). In project II (see section 2.2 on page 40), a MRI compatible experimental set up is designed. This setup improves upon previously employed setups (such as implemented by Culham et al., 2003; Cavina-Pratesi et al., 2007, 2010b, Nelissen and Vanduffel, 2011, Nowik et al., 2019).

The final project III (see section 2.3 on page 63) investigates prehension in humans and macaques utilising the set-up designed in project II. Unlike the work of CavinaPratesi et al. (2018), it separates reach and grasp not by comparing reach and pointing actions, but by having a grasp only and a reach-to-grasp condition. The often employed pointing action as a placeholder for reach actions is highly questionable (Culham et al., 2006). Additionally, the advantages of the improved experimental design are utilized to investigate the control of prehension in time. By manipulating the predictability of the go-cue, the moment of initiation of the prehension action is manipulated, while the onset of the movement execution is kept constant in relation to the trial and small differences in the timing of the movement execution by the subject are removed in the analysis, allowing the separation of both processes. 


\section{Original Projects}

\subsection{Project I: Shared functional connectivity between the dorso-medial and dorso-ventral streams in macaques.}

\section{Author contributions}

R.S. Greulich and H. Scherberger conceived the research; S. Everling and R. Adam contributed data; R.S. Greulich and R. Adam designed the data analysis. R.S. Greulich performed the data analysis. R.S. Greulich wrote the manuscript. R.S. Greulich, R. Adam, S. Everling, and H. Scherberger revised and approved the final version of the manuscript.

\subsubsection{General Discussion}

The first project aimed to bring further insight into the separation between networks utilised to control grasp and reach actions, since the science is still open on whether reach and grasp are controlled in two separate streams or in one unified network (see Jeannerod, 1981; Galletti et al., 2003; Fattori et al., 2004, and others cited in the publication).

We used resting state $\mathrm{fMRI}$ and optimal modularity as unbiased clustering algorithm to investigate this question (Newman, 2006). Resting state fMRI allows the examination of the network properties of the whole brain. Optimal modularity, as the used clustering algorithm, permits to statistically significant separate the correlation matrix between examined areas into one or multiple networks, or even, if the modularity of the optimal network is not statistically significant from chance, to give no definite answer to the question. This methodological approach has so far not been implemented to investigate the given research question and, as such, allows new insights free of possible confounds that the previously used methods might suffer from. Since this study is investigating a previously considered research question with new 
methodology, it is a replication study of the concept replication type with the context subtype (Dennis and Valacich, 2014, Brendel et al., 2020). Proper replication studies are vital to the field of neuroscience, which is suffering from the replication crisis as much as other fields (Barch and Yarkoni, 2013).

The study utilizes a high-quality data set consisting of 10 macaques examined with a $7 \mathrm{~T}$ scanner, a custom head coil and an $2 \mathrm{x}$ multiband sequence. We picked motor and premotor areas which have previously been examined by various other studies into motor control of arm and hand. For the reach or dorso-medial stream, we picked the areas V6A, MIP and F2 (Tanné-Gariépy et al., 2002a Gamberini et al., 2011; Hwang et al., 2012). In the grasp or dorso-ventral stream, we selected AIP. F5 and hand area of the primary motor cortex ( $\left.\mathrm{M} 1_{\text {hand }}\right)$ (Schaffelhofer and Scherberger, 2016). We examined the inter-connectivity of those areas with a seed based approach.

The results are in favor of the two-stream hypothesis. The correlation matrix between the selected areas clearly and significantly separates into two networks. One network includes $\mathrm{AIP}, \mathrm{F} 5$, and $\mathrm{M} 1_{\text {hand }}$ and constitutes the dorso-ventral network. The other consists of V6A, MIP and F2 and represents the dorso-medial network. Significant connections linking both networks are between MIP,AIP, V6A,AIP, and M1 $1_{\text {hand }}$ F2.

Additionally, we performed a whole brain correlation analysis for each area and examined the overlaps and differences between both networks. The dorso-ventral network was exclusively connected with high order somatosensory and feeding associated areas such as gustatory cortex, precentral opercular area $(\overline{P r C O})$ and area PF. This association makes sense intuitively, as feeding is one of the most common uses for a grasp in a macaque's life (Napier et al., 1967). Additionally, a grasp needs somatosensory information about object parameters, such as weight, surface, and shape, to maintain the correct grasp (Leonard et al., 1992).

The dorso-medial network showed exclusive connections with visual and hind limb and trunk motor areas. The position of an object in space is much more important for the reach than for a grasp. Since a reach for an object cannot rely on somatosensory information, as said information is not present before an object is touched, it relies on visual information (Winges et al., 2003; Rand et al., 2007). To reach an object outside of the immediate personal space of the animal, a movement of the trunk or the hind limbs are necessary, explaining the connectivity to those areas.

However, both networks also show interconnectivity, with areas along the intraparietal sulcus, part of area PFG, area PGop, and FEF being connected to both networks. Those areas are mainly associated with the coordination of visualmotor actions as well as high order integration of multimodal sensory input and motor actions. 
This work was published in Scientific Reports (Greulich et al., 2020) on October $29^{\text {th }}, 2020$ and the publication is included as published.

However, this study could not answer if and how both networks are engaged during an actual reach and grasp action. Additionally, the question remains, if this network separation is true for humans as well as for macaques. Also, the interplay between reach and grasp are still unclear. To answer this question for both species, an experiment needs the technical requirements for a grasp and reach-to-grasp paradigm. The following project solves the technical requirements for such an experiment utilizing fMRI, while the third project implements it. 


\title{
scientific reports
}

Check for updates

\section{OPEN Shared functional connectivity between the dorso-medial and dorso-ventral streams in macaques}

\author{
R. Stefan Greulich ${ }^{1,5 \bowtie}$, Ramina Adam ${ }^{2,3}$, Stefan Everling ${ }^{2,4}$ \& Hansjörg Scherberger ${ }^{1,5 \bowtie}$
}

Manipulation of an object requires us to transport our hand towards the object (reach) and close our digits around that object (grasp). In current models, reach-related information is propagated in the dorso-medial stream from posterior parietal area V6A to medial intraparietal area, dorsal premotor cortex, and primary motor cortex. Grasp-related information is processed in the dorsoventral stream from the anterior intraparietal area to ventral premotor cortex and the hand area of primary motor cortex. However, recent studies have cast doubt on the validity of this separation in separate processing streams. We investigated in 10 male rhesus macaques the whole-brain functional connectivity of these areas using resting state fMRI at 7-T. Although we found a clear separation between dorso-medial and dorso-ventral network connectivity in support of the two-stream hypothesis, we also found evidence of shared connectivity between these networks. The dorsoventral network was distinctly correlated with high-order somatosensory areas and feeding related areas, whereas the dorso-medial network with visual areas and trunk/hindlimb motor areas. Shared connectivity was found in the superior frontal and precentral gyrus, central sulcus, intraparietal sulcus, precuneus, and insular cortex. These results suggest that while sensorimotor processing streams are functionally separated, they can access information through shared areas.

The two-stream hypothesis regarding hand and arm motor actions has been discussed since the early $1980 \mathrm{~s}^{1}$ (for review $\mathrm{see}^{2}$ ). It postulates that information processing for reach and grasp actions are implemented in two distinct cortical streams, in the dorso-ventral stream for grasp processing and in the dorso-medial stream for reaching (for review $\mathrm{see}^{3}$ ). The dorso-medial pathway extends from the primary visual cortex over V6A, to the medial intraparietal area (MIP) and to the dorsal premotor cortex (also known as area F2), while the dorso-ventral pathway goes over the anterior intraparietal area (AIP) towards the ventral premotor cortex (in particular: area $\mathrm{F} 5)^{4}$ and the primary motor cortex (M1), which is well supported by tracer studies ${ }^{5-14}$.

There is direct causal evidence for the separation of both circuits in the form of chemically or magnetically induced lesions in both humans and non-human primates. For example, inactivation of area F5 and AIP is associated with strong deficits on grasping, but no effect on reaching movements, both in macaques ${ }^{15,16}$ and human $\mathbf{s}^{17,18}$. In contrast, damage to MIP and V6A, which are often summarized as the parietal reach region $\mathrm{PRR}^{19}$, leads to a condition known as optic ataxia that comprises reach deficits as well as minor grasp impairments that are likely a consequence of the patient's reach uncertainty ${ }^{20}$. Finally, electrophysiological recording experiments in nonhuman primates confirmed specialized functions of these areas consistent with the two-stream hypothesis ${ }^{6,21-26}$.

However, some studies have found grasp-related activity in traditional reach-related areas and reachrelated activity in traditional grasp-related areas, contradicting the notions of a strict separation between both streams $s^{27,28}$. Activity related to hand orientation was found in $\mathrm{F}^{29}$ and in $\mathrm{V}^{2} \mathrm{~A}^{30}$, both areas part of the reachrelated dorso-medial stream, while reach activity was found in $\mathrm{F}^{31}$, an area part of the grasp-related dorsoventral stream. Other studies added support for these findings ${ }^{32-36}$, warranting further investigation into the separation between reaching and grasping processes in the brain.

\footnotetext{
${ }^{1}$ Deutsches Primatenzentrum GmbH, Kellnerweg 4, 37077 Göttingen, Germany. ${ }^{2}$ Robarts Research Institute, University of Western Ontario, London, Canada. ${ }^{3}$ Graduate Program in Neuroscience, University of Western Ontario, London, Canada. ${ }^{4}$ Department of Physiology and Pharmacology, University of Western Ontario, London, Canada. ${ }^{5}$ Faculty of Biology and Psychology, University of Goettingen, Göttingen, Germany. ${ }^{\circledR}$ email: sgreulich@ dpz.eu; hscherberger@dpz.eu
} 
We aimed to resolve whether grasping and reaching functions recruited two distinct functional cortical networks and employed a functional connectivity analysis of resting state functional magnetic resonance imaging (rs-fMRI) data. Unlike tracer studies, rs-fMRI is not limited to monosynaptic connections, but rather allows for the added identification of functionally connected areas through polysynaptic connections. Correlation between areas gives a measurement of how strong they are functionally interlinked ${ }^{37}$. Paired with modern cluster detection $^{38}$, this approach allows us to discern whether the cortical areas F2, F5, M1, AIP, V6A and MIP belong to one or multiple networks. Also, unlike electrophysiological experiments, it allows us to study the whole brain and explore the extension of the resulting networks. We analyzed rs-fMRI data in a population of 10 lightly anesthetized macaque monkeys with seeds placed in six cortical areas in the dorso-medial and the dorso-ventral stream. We show that both processing streams form clearly separated functional networks, however, there are specific areas to which both networks are connected, suggesting a possible communication link between the dorso-medial and dorso-ventral network.

\section{Materials and methods}

Resting state fMRI data was collected from 12 male adult rhesus macaque monkeys (Macaca mulatta). Of these, two animals were excluded, one because of a susceptibility artifact over the right parietal lobe and one because of an abnormally shaped central sulcus. The remaining 10 animals had a body weight of $6.1-11.8 \mathrm{~kg}$ (mean: 8.1, std: 1.6) and were 5-10 years of age (mean; 6.2, std: 1.7). Animal care and experimental procedures followed the guidelines of the Canadian Council on Animal Care policy on experimental animals. All procedures were approved by the Institutional Animal Care Committee of the University of Western Ontario (Animal Use Protocol Number 2008-125), and all animal experiments were conducted there.

Anesthesia. Animals were first sedated with $0.1-0.2 \mathrm{mg} / \mathrm{kg}$ acepromazine, followed by $7.5 \mathrm{mg} / \mathrm{kg}$ ketamine hydrochloride by intramuscular injection, before anesthesia was induced with $2.5 \mathrm{mg} / \mathrm{kg}$ propofol via an intravenous catheter in the saphenous vein. Anesthesia was maintained with 1-2\% isoflurane with oxygen (1.5-2 l/ $\mathrm{min}$ ) through endotracheal intubation, which was reduced to $1 \%$ during resting-state functional imaging. Heart rate, $\mathrm{O}_{2}$-saturation, respiration rate and respiratory $\mathrm{CO}_{2}$ levels were continuously monitored.

Data acquisition and preprocessing. Macaque monkeys were scanned in a $7 \mathrm{~T}$ Scanner (Siemens, Erlangen, Germany) with a $40 \mathrm{~cm}$ gradient coil with field strength of $80 \mathrm{mT} / \mathrm{m}$. A custom build 24-channel phased array head-coil ${ }^{39}$ was used to collect 2 -dimensional multi-band $\mathrm{T} 2{ }^{*}$ weighted EPI images $(\mathrm{TR}=1000 \mathrm{~ms}$, $\mathrm{TE}=18 \mathrm{~ms}$, flip angle $=40^{\circ}, 42$ slices, resolution $1 \times 1 \times 1.1 \mathrm{~mm}$, FOV $96 \times 96 \mathrm{~mm}$, and matrix size $96 \times 96$ ). In total 4 runs of 600 functional volumes were recorded sequentially in one session. Furthermore, standard T1-weighted anatomical images were acquired in the same orientation with $0.5-\mathrm{mm}$ isotropic resolution.

Data was preprocessed with the software package FSL (fMRI Software Library: https://www.fmrib.ox.ac.uk). Functional images were corrected for motion and image acquisition timing. High pass filtering was implemented by subtracting a Gaussian least-square straight-line fit (sd: $100 \mathrm{~s}$ ) and subsequent low-pass filtering with Gaussian smoothing (sd: $2.8 \mathrm{~s}$ ). Brain extraction was done with the Brain Extraction Tool and the BrainSuite toolbox ${ }^{40}$. Average EPI images were realigned to the anatomical scans and both co-registered with the standard F99 atlas ${ }^{41}$. Finally, functional images were spatially smoothed (Gaussian filter, FWHM: $3 \mathrm{~mm}$ ). Further details of the scanning protocol and data preprocessing are included $\mathrm{in}^{42}$. Unless stated otherwise, this publication follows the parcellation of the macaque cortex as introduced by ${ }^{43}$.

Experimental design and statistical analysis. All analyses were based on data from 10 macaque monkeys, as detailed above. Statistical procedures were performed on individual subjects as well as across the subject population, as described in the following sections.

Seed-based correlation analysis. A seed-based correlation analysis was performed with extracted time series from six individual seeds in both hemispheres. All seeds were placed using the Saleem and Logothetis atlas as a reference ${ }^{44}$ in the F99 brain template ${ }^{45}$ (Fig. 1). Seeds in AIP, F5 and $\mathrm{M1}_{\text {hand }}$ were positioned according to the location of implants in ${ }^{46}$ as guidance to locations with known involvement in grasp planning and execution. Further seeds were placed in V6A and MIP according to locations provided by ${ }^{34}$ and $^{19}$, respectively. F2 seed was placed according to injection site reported in $^{8}$. See supplemental methods for a more extensive description of the seed locations and supplemental Table S1 for the precise coordinates. Seeds had a radius of $1 \mathrm{~mm}$ and encompassed 7 voxels. The minimal distance between seeds was between V6A and MIP with $10 \mathrm{~mm}$. Each brain was normalized to the F99 template with FSL, eliminating the need of individual seed placement.

Time series for each seed, cerebrospinal fluid (CSF) and white matter (WM) were extracted for each monkey and run. We calculated whole-brain correlations for each monkey with a general linear model with CSF and WM included as regressor of noninterest. The resulting correlation maps for each animal were then included in a population analysis for each seed individually calculated and modeled independently with a mixed effects model (threshold: $\mathrm{p}=0.05$, voxel-wise corrected for multiple comparison with GRF-theory-based maximum height thresholding as implemented in FSL).

Modularity analysis between seeds. For the between seed comparison, correlation coefficients between the extracted time series were calculated and averaged over runs in each individual subject. Mean and variance across individuals was reported in the correlation matrix. These values were tested for significant difference from zero with a two-sided $t$ test with Bonferroni correction for multiple testing. To test whether connections separate 
AIP
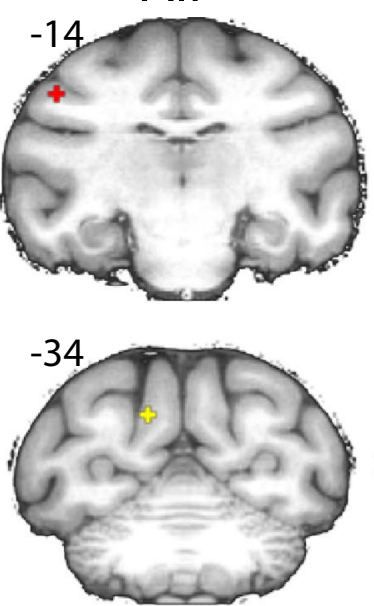

V6A
F5
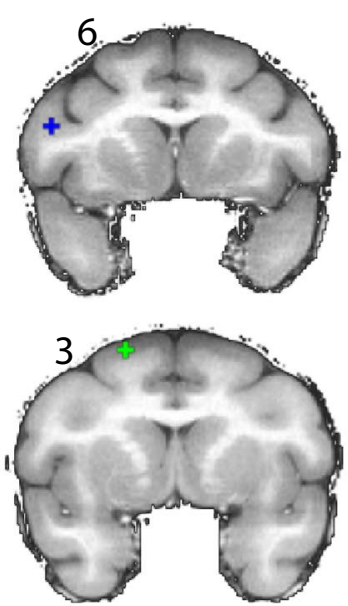

F2
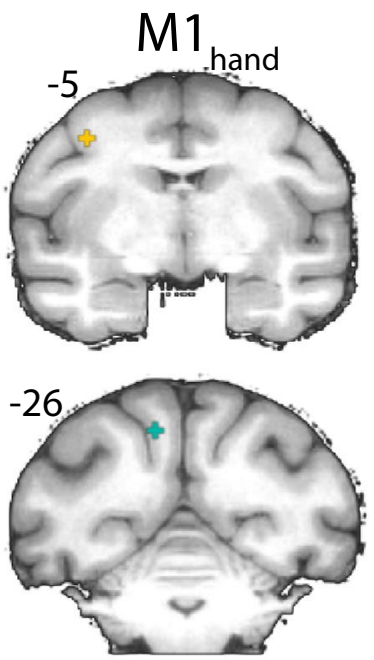

MIP

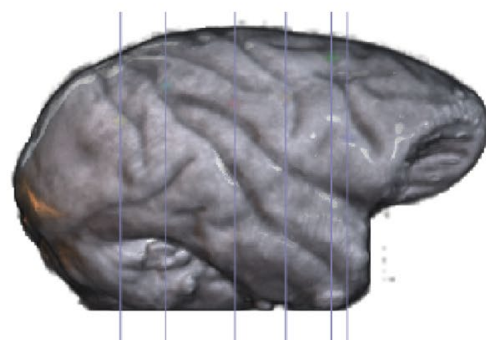

$-34-26-14-536$

Figure 1. Placement of the seeds in the F99 brain template ${ }^{45}$. Top row, left to right: AIP, F5 and $\mathrm{M} 1_{\text {hand. }}$ Bottom row, left to right: V6A, F2 and MIP. Y coordinates of the sections are given relative to the F99 reference frame ${ }^{45}$. Sections rendered with MRIcoGL ver. 1.2.20181114 + (https://www.mccauslandcenter.sc.edu/mricrogl/).

into distinct networks, we assessed the optimal modularity ${ }^{38}$, as implemented in the brain connectivity toolbox ${ }^{47}$, and tested for significance $(\mathrm{p}<0.05)$ against the modularity of $1,000,000$ randomly shuffled, symmetrical connectivity matrices. Optimal modularity is especially well suited for this research question as the algorithm stops if a network is considered indivisible. It therefore does return an unbiased division into one or more networks ${ }^{38}$.

Conjunction analysis between seeds. Since all cortical areas show neural correlation with a grasp process ${ }^{48}$, a simple correlation analysis over all seeds together is not applicable since the resulting contrast vectors would not be orthogonal and a simple addition would lead to exaggerated results. Therefore, whenever correlation maps of a network, detected by the modularity analysis, were combined, we used a much stricter approach: only voxels that were significant in each individual seed map (FDR corrected $\mathrm{z}$-score $>2.3$ ) were included in the conjunction map. The results from the individual seed map were converted to a binary mask with a voxel mapped to 1 if it was significantly correlated with the seed or 0 otherwise. Those binary maps were then summed according to the detected networks in the modularity analysis. A similar method, reporting voxels with 2 out of 3 significant tests, was used in $^{49}$. The result is then mapped onto the brain with each voxel value representing in how many seed maps the voxel is significant. We considered only voxels, which were significantly correlated towards all the seeds of a network, to be part of said network.

\section{Results}

Using rs-fMRI of 10 lightly anesthetized macaque monkeys, we obtained connectivity maps by placing seeds in each of the cortical areas AIP, M1 $1_{\text {hand }}$ and F5 of the dorso-ventral stream as well as in F2, V6A and MIP of the dorso-medial stream. For each animal, four rs-fMRI runs were averaged and results from all animals were combined in a multi-effect analysis (see Materials and Methods). In the following section, we first report connectivity maps for individual seed areas and then take a more global approach by generating maps from multiple seeds.

Results from seeds placed in the left or right hemisphere were largely symmetrical. We therefore report only functional connectivity results of the left seeds in both hemispheres. For the results from the seeds in the right hemisphere see Figure S1 and S2 in the supplementary material. Furthermore, since the RF coil used here is known to have a low signal-to-noise ratio in the cerebellum and brainstem, we only report results from cortical and subcortical areas, even though some significant correlations were also found in the cerebellum. Significant results in the midbrain are reported, but due to the low signal, they should be interpreted with caution.

Functional connectivity map of AIP. The connectivity map of left AIP covers large portions of the parietal lobe, including posterior regions up to the superior temporal sulcus, intraparietal sulcus and the parietooccipital sulcus (Fig. 2, top panels). In anterior regions, the main correlation cluster reaches up to the arcuate sulcus; however, the cingulate cortex also shows correlation. Additionally, there is correlation in area 13 and lateral area 12. Correlations peak in the cingulate sulcus, the anterior part of the intraparietal sulcus and the middle part of the central sulcus. All these correlations are symmetrical across the left and right hemisphere. Asymmetries appeared in the activation of the superior arcuate sulcus, with a stronger correlation and with a more anterior peak in the right hemisphere. Furthermore, there was stronger correlation on the left side, as compared to the right, in the internal part of the lateral fissure around the internal secondary somatosensory cortex. Additionally, 

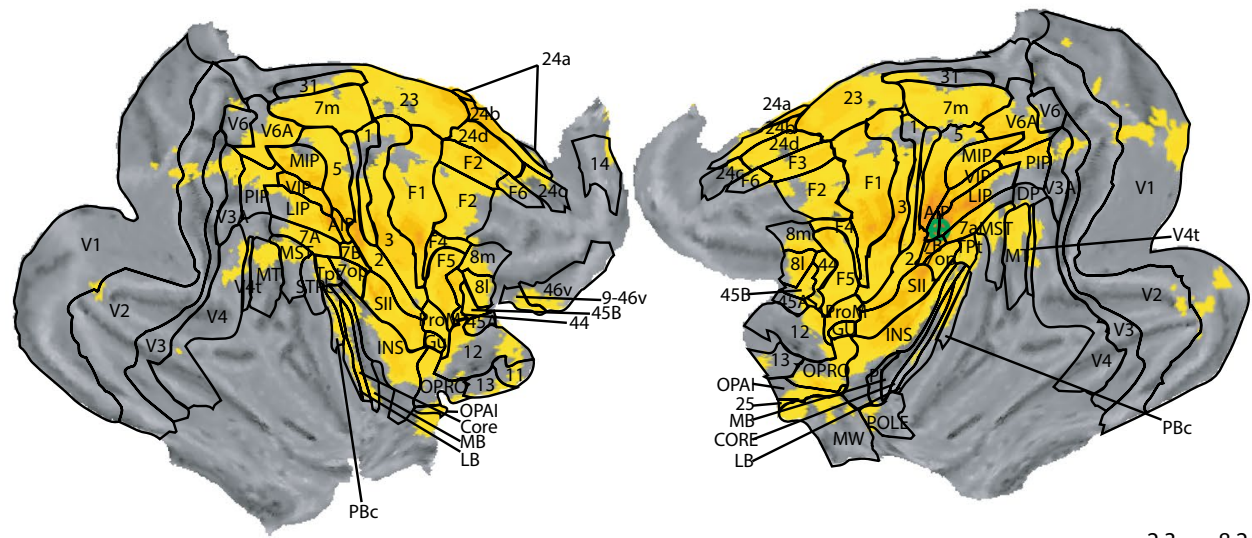

F5
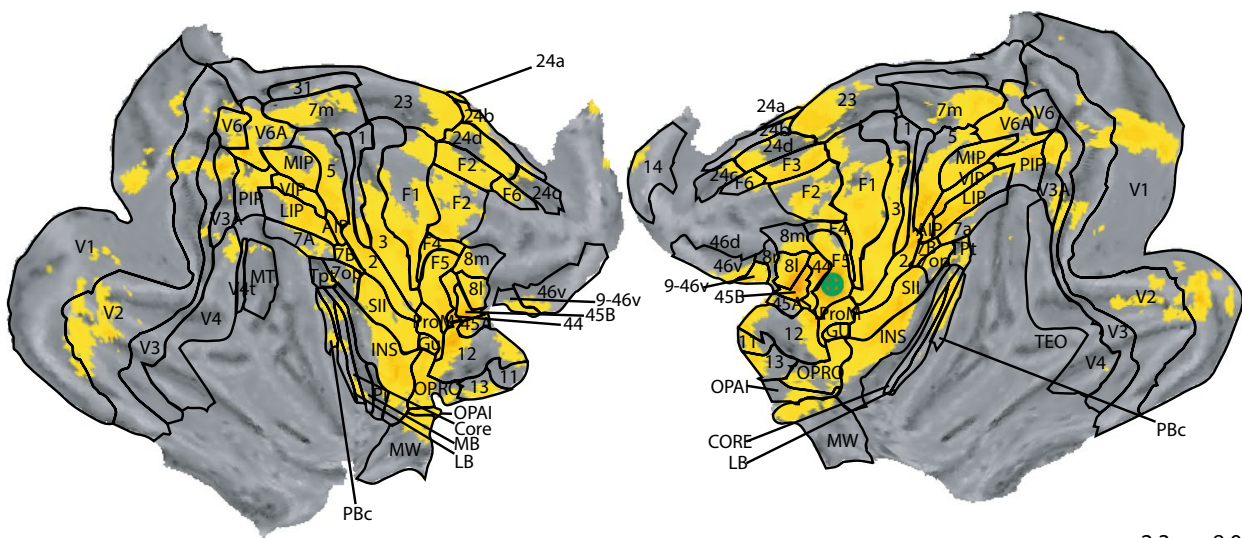

$M 1_{\text {hand }}$
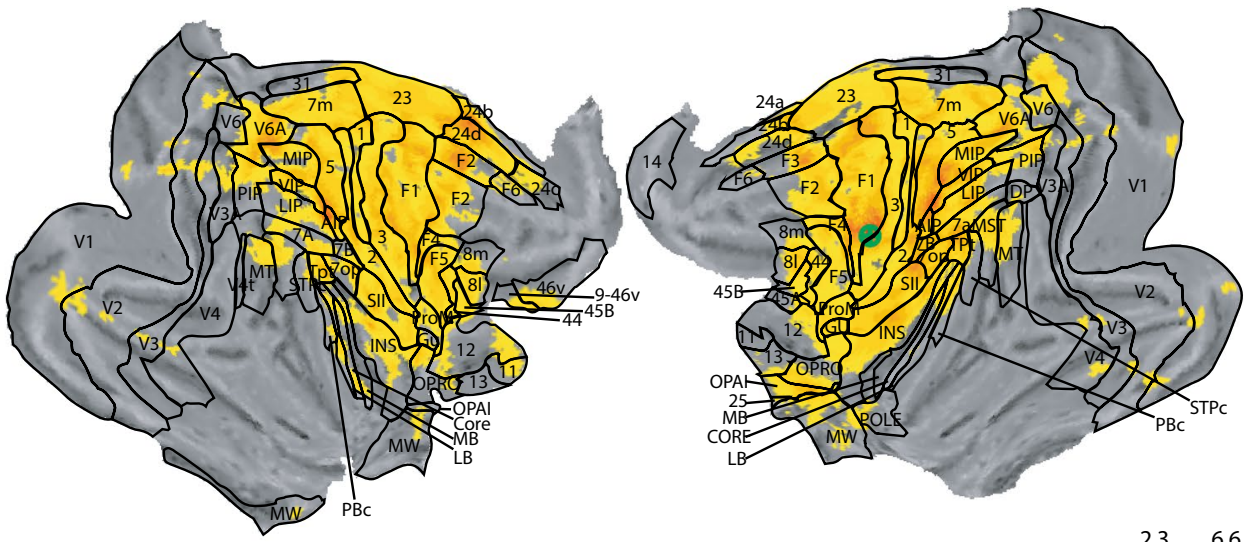

Figure 2. Whole brain correlation of the AIP, $\mathrm{F} 5$ and $\mathrm{M}_{1}$ hand seeds, projected onto the cortical surface. $\mathrm{z}$-statistic maps are FDR corrected and thresholded according to the z-score color bar. Please note that each seed has a different $z$-score scaling for the projection. Seed placement is marked by the green marker on the left hemisphere. Since the analysis was done on the 3D dataset, the seed placement marking is approximated. All surface renderings (flat maps) were done with CARET v5.65 (https://brainvis.wustl.edu/wiki/index.php/Caret :About $)^{88}$. Cortical area labeling and borders according to ${ }^{43}$. 
there was significant correlation extending from area 46 on the right side. On the left side, we found correlation in middle temporal area.

Concerning subcortical areas, we found significant correlation in the putamen, external globus pallidus, and the stria medullaris (Fig. 3, top panels). The thalamic nuclei also showed significant correlations, especially the deep mesencephalic nuclei, the body as well as posterior parts of the head of the caudate nucleus, medial geniculate nucleus, and the posterior part of the caudate nucleus.

Functional connectivity map of ventral premotor cortex (area F5). When placing seeds in area F5, as part of the ventral premotor cortex, we found strong functional connectivity with the parietal lobe (Fig. 2, middle panels). The posterior border of the correlation map followed the intraparietal sulcus and the lateral fissure, together with some significant correlation at the cuneate gyrus and the superior parietal lobe. Anteriorly, the main cluster extended to the superior arcuate sulcus and at the height of the AC-PC line all the way to the front of the brain. Furthermore, there was a bilateral significant correlation following the principal sulcus, the intraprincipal dimple and the medial orbital sulcus. Cortically, the strongest correlations were located at the right superior temporal sulcus, area 2 , the right agranular insular cortex, bilateral in the dysgranular insular cortex, area $45 \mathrm{~B}$, opercular parietal area PF (as defined by ${ }^{50}$ roughly equivalent to area $7 \mathrm{~b}$ in the parcellation of ${ }^{43}$ ), visual area V3A, and at the anterior part of the intraparietal sulcus and the insular cortex. Significant unilateral correlation was found in the right middle temporal area (V5), whereas in the cingulate cortex correlation was stronger on the left vs. the right hemisphere.

In subcortical structures, significant correlations appeared mostly bilaterally in the putamen, the anterior and posterior limb of the internal capsule, the external capsule and the claustrum, except for the mostly left-lateralized caudate nucleus (Fig. 3, middle panels).

Functional connectivity map of primary motor cortex. The correlation map of the hand area of M1 also covered the parietal lobe (Fig. 2, bottom panels), with the posterior border of significant correlation marked by the parieto-occipital sulcus, the superior temporal sulcus, and the lateral fissure ventral to the anterior commissure (AC)-posterior commissure (PC) line. Anteriorly, the main cluster of significant correlation reached up to the spur of the arcuate sulcus. In the cingulate sulcus, the cluster extended up to a height of the anterior end of the arcuate sulcus. Here as well, the strongest correlations manifested in the cingulate sulcus, the intraparietal sulcus and the central sulcus. Most of the correlation was bilateral, with the left hemisphere demonstrating higher values than the right one. However, significant correlation was unilateral at the left side of the agranular insular cortex, extending down to the piriform cortex, and on the left side in the lateral area 12, visual area 2, and the medial superior temporal area (MST).

Subcortically, the dorsal part of the thalamic nuclei and part of the fornix, showed significant correlation, as well as the lenticular fasciculus, anterior pulvinar, internal capsule, claustrum, putamen, and the caudate nucleus (Fig. 3, bottom panels). In terms of intensity, the left globus pallidus and the left nucleus accumbens demonstrated stronger correlations than the corresponding right-hemispheric structures.

Functional connectivity map of area V6A. The correlation map for area V6A covered large areas around the secondary visual cortex and the dorsal half of the parietal and frontal lobe (Fig. 4, top panels). Correlations focused mainly around the cuneus and annectant gyrus and up to the dorsal part of the occipital gyrus, while the lunate sulcus and the calcarine sulcus formed the anterior border of significant correlation. The lingual and the fusiform gyrus also showed prominently correlations. From the posterior end of the lateral sulcus to the anterior end of the intraparietal sulcus, virtually all grey matter was significant, with the fusiform gyrus being the only exception. Furthermore, the cingulate gyrus was significantly correlated along its entire length, the superior temporal gyrus showed significance in its posterior half, whereas at the anterior end of the intraparietal sulcus, only the insula, middle temporal gyrus, and the precentral gyrus showed significant correlation. In the frontal lobe, the superior and middle frontal gyrus showed significant correlations. In general, there was not much deviation from symmetry.

Subcortical correlations were found mainly in the caudate nucleus, internal capsule, thalamic nuclei, medial pulvinar, and the putamen (Fig. 5, top panels). Results were mainly symmetrical, with exceptions in the frontal part of the claustrum, which had no correlations on the right side, and in the right thalamic nuclei that had stronger and more widespread correlations than in the left.

Functional connectivity map of MIP. Functional correlation of MIP focused on the visual cortex and the dorsal part of the parietal and frontal lobe, and also prominently featured the cuneus (Fig. 4, middle panels). Significant correlations were also found in the supramarginal and lingual gyrus as well as in the insula. The superior frontal and cingulate gyrus were significant up to their frontal margin. In the frontal lobe, there was strong correlation in area 46 and F2, however, more strongly on the right side than the left. We also saw significant correlations in area 12. All of these correlations were bilateral. Furthermore, there were significant unilateral correlations in the right area 13 and the right somatosensory areas $3 \mathrm{~b}$ and 2. Posterior to AC, correlations were generally stronger on the left side, while anterior to AC they were stronger on the right side, except for the right fasciolar gyrus. Furthermore, we found significant correlations with the left primary visual cortex.

MIP was also significantly correlated with a number of subcortical structures, most prominently the superior colliculus and the medial and the left oral pulvinar, as well as the putamen and claustrum (Fig. 5, middle panels). Significant correlations were also found in the caudate nucleus anterior to the AC and in the ventral part of the internal capsule and globus pallidus. 
AIP
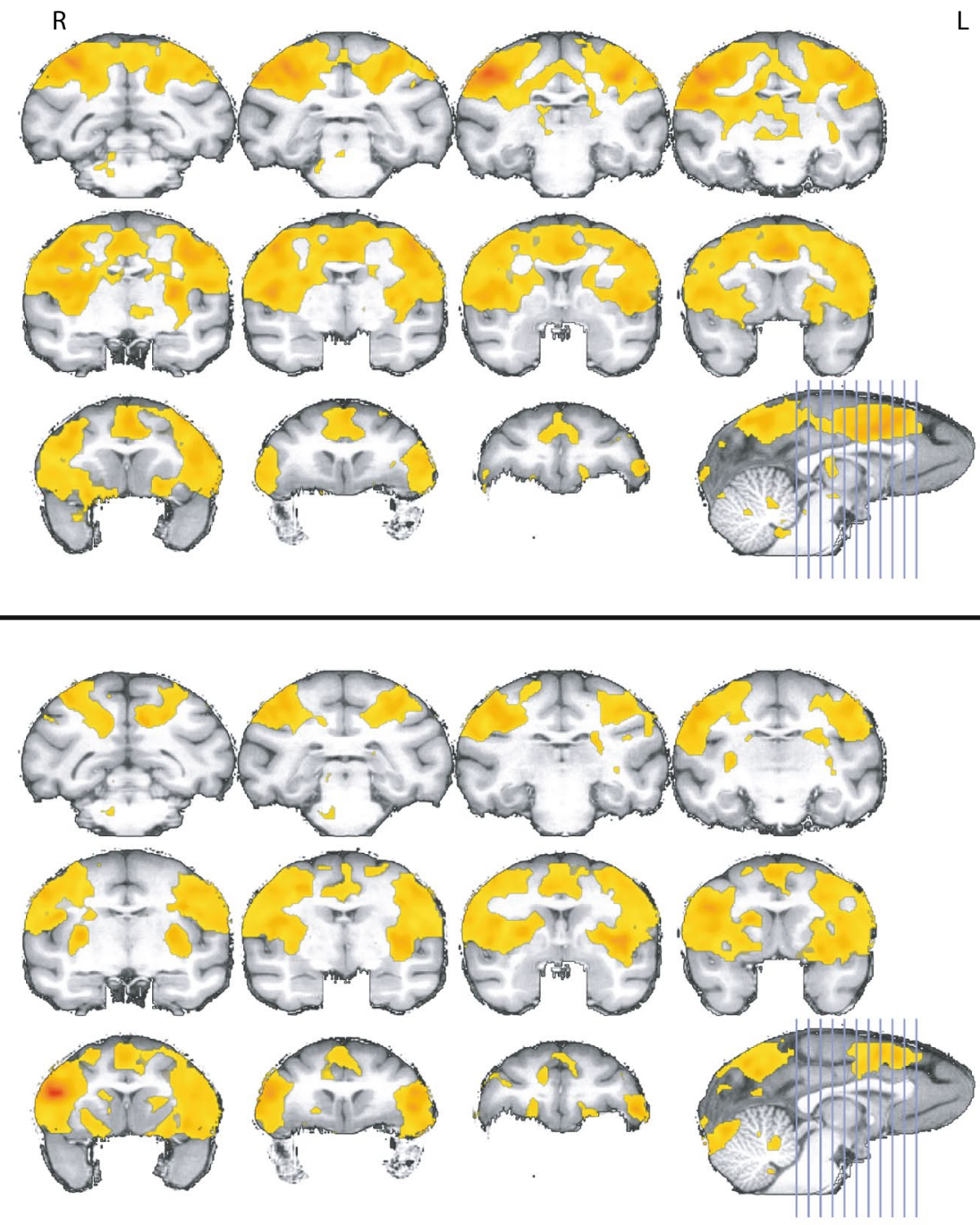

$M 1_{\text {hand }}$

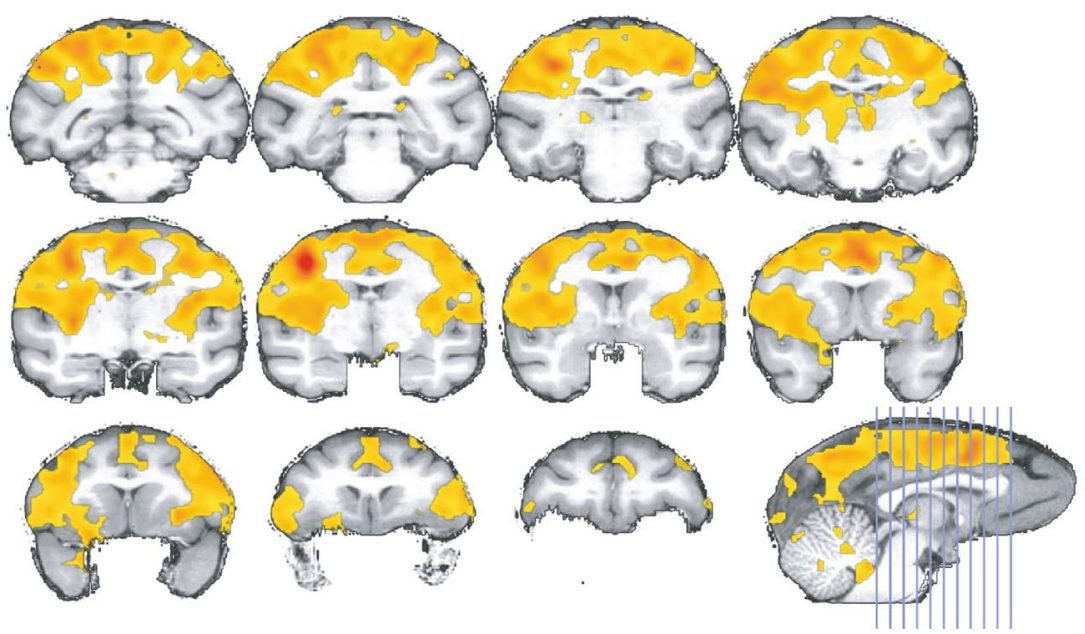

2.3 6.6

Figure 3. Correlation for AIP, $\mathrm{F} 5$ and $\mathrm{M} 1_{\text {hand }}$ in the deep brain structures. Z-statistics thresholded according to the individual z-score color bar. All conventions as in Fig. 2. Y coordinates of the sections are given relative to the F99 reference frame ${ }^{45}$. Sections rendered with MRIcoGL ver. 1.2.20181114+ (https://www.mccauslandcente r.sc.edu/mricrogl/). 
V6A
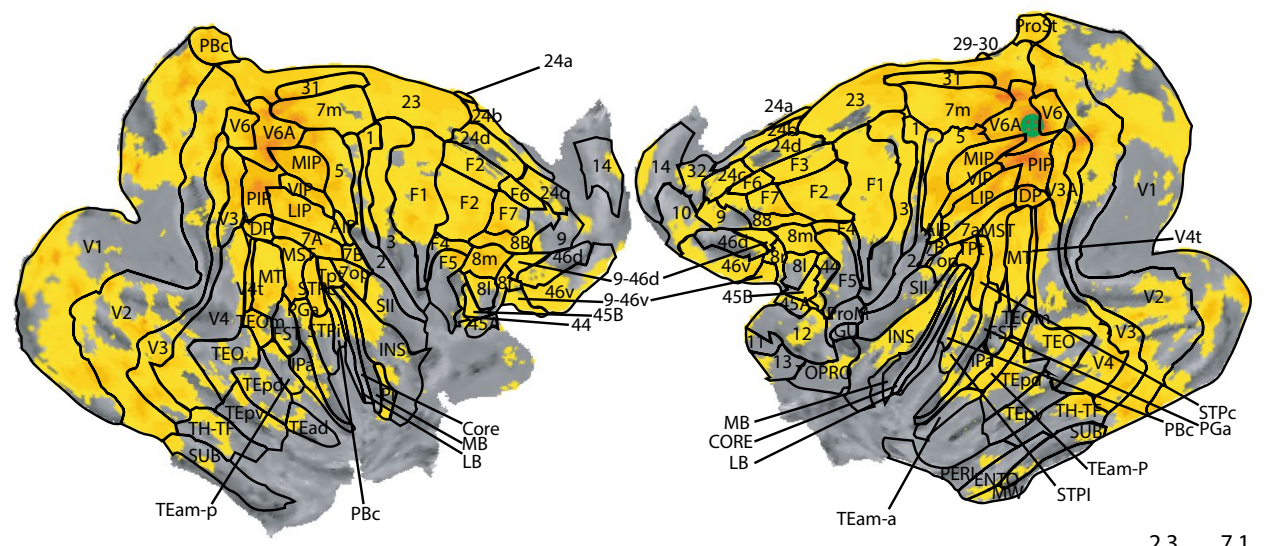

MIP

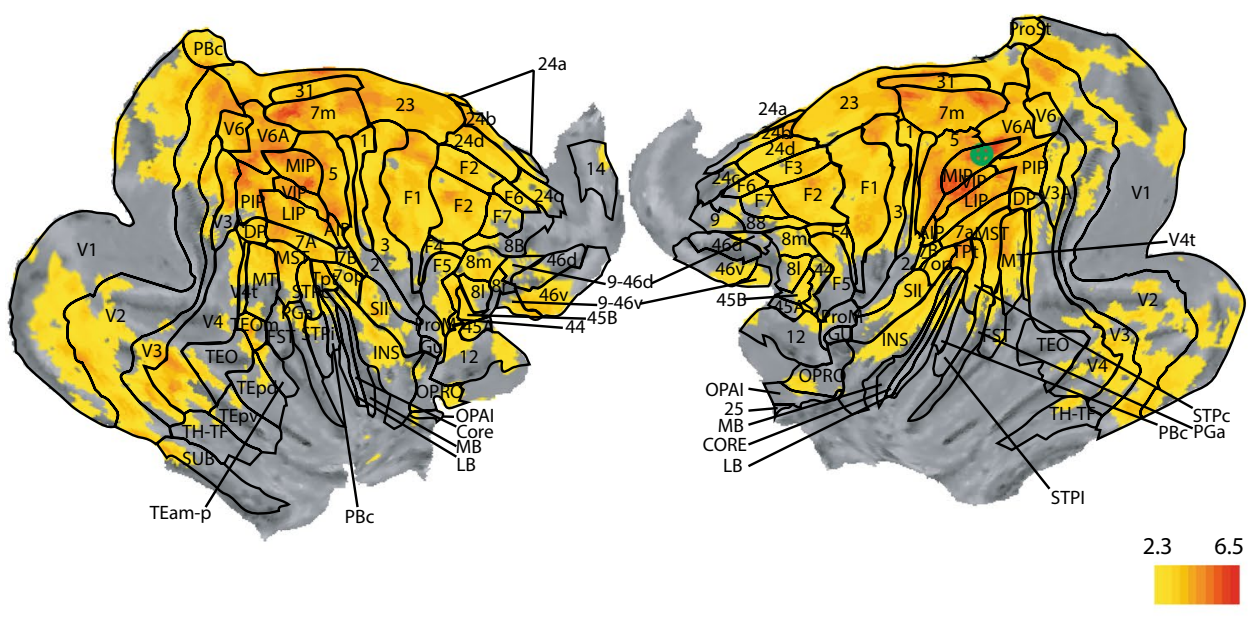

F2
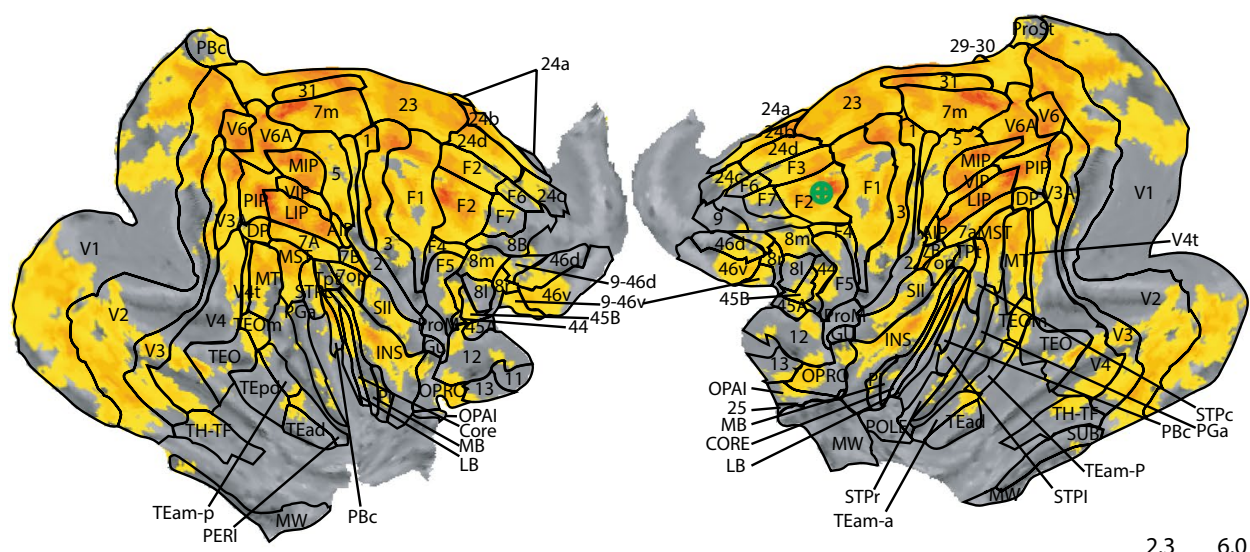

$2.3 \quad 6.0$

Figure 4. Whole brain correlation of the V6A, MIP and F2 seeds, projected onto the cortical surface. z-statistic maps are FDR corrected and thresholded according to the z-score color bar. Please note that each seed has a different $\mathrm{z}$-score scaling for the projection. Seed placement is marked by the green marker on the left hemisphere. Since the analysis was done on the 3D dataset, the seed placement marking is approximated. All surface renderings (flat maps) were done with CARET v5.65 (https://brainvis.wustl.edu/wiki/index.php/Caret :About ${ }^{88}$. Cortical area labeling and borders according to ${ }^{43}$. 
V6A
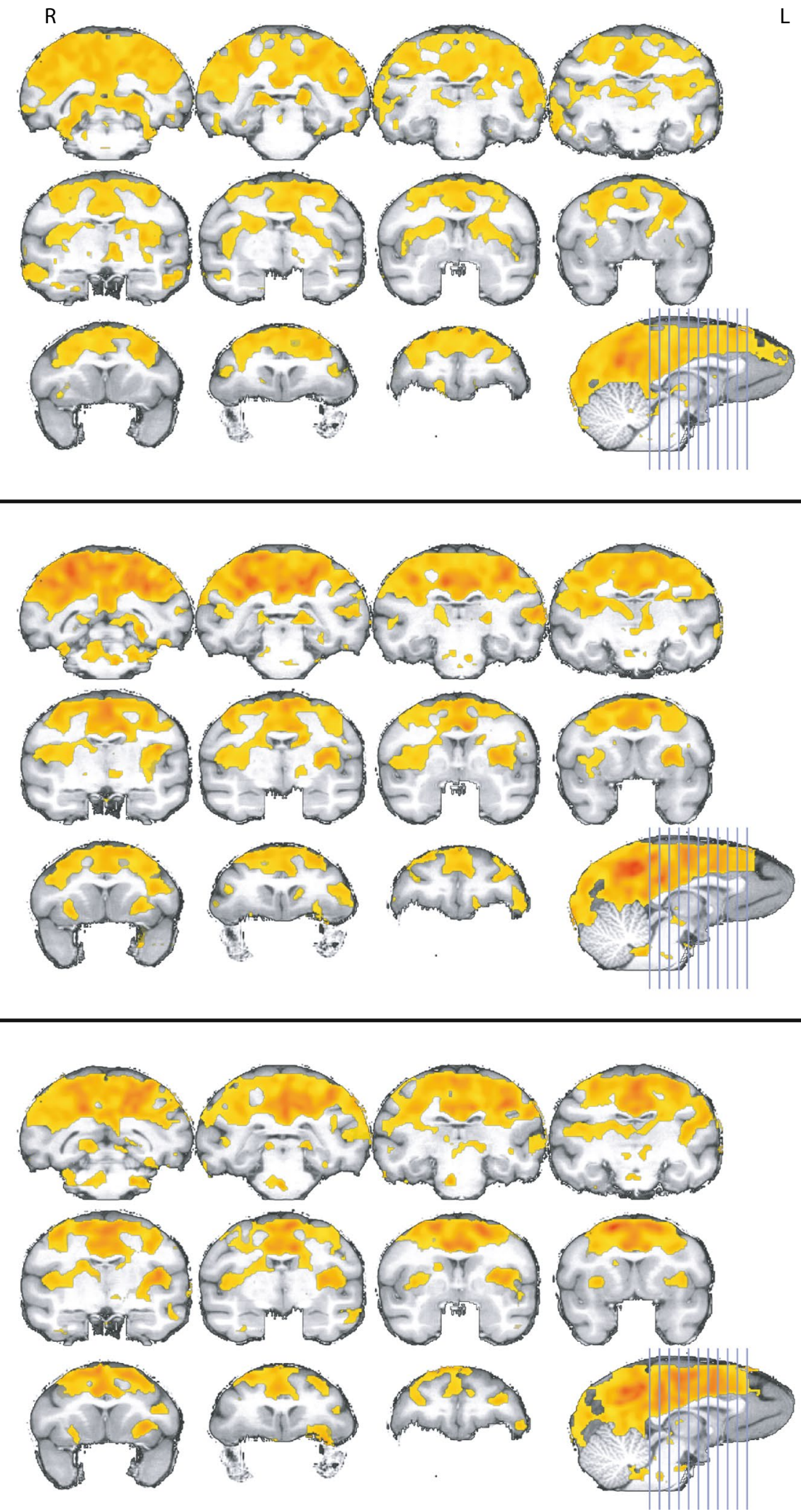

Figure 5. Correlation for V6A, MIP and F2 in the deep brain structures. Z-statistics thresholded according to the individual z-score color bar. All conventions as in Fig. 4. Y coordinates of the sections are given relative to the F99 reference frame ${ }^{45}$. Sections rendered with MRIcoGL ver. 1.2.20181114+ (https://www.mccauslandcente r.sc.edu/mricrogl/). 


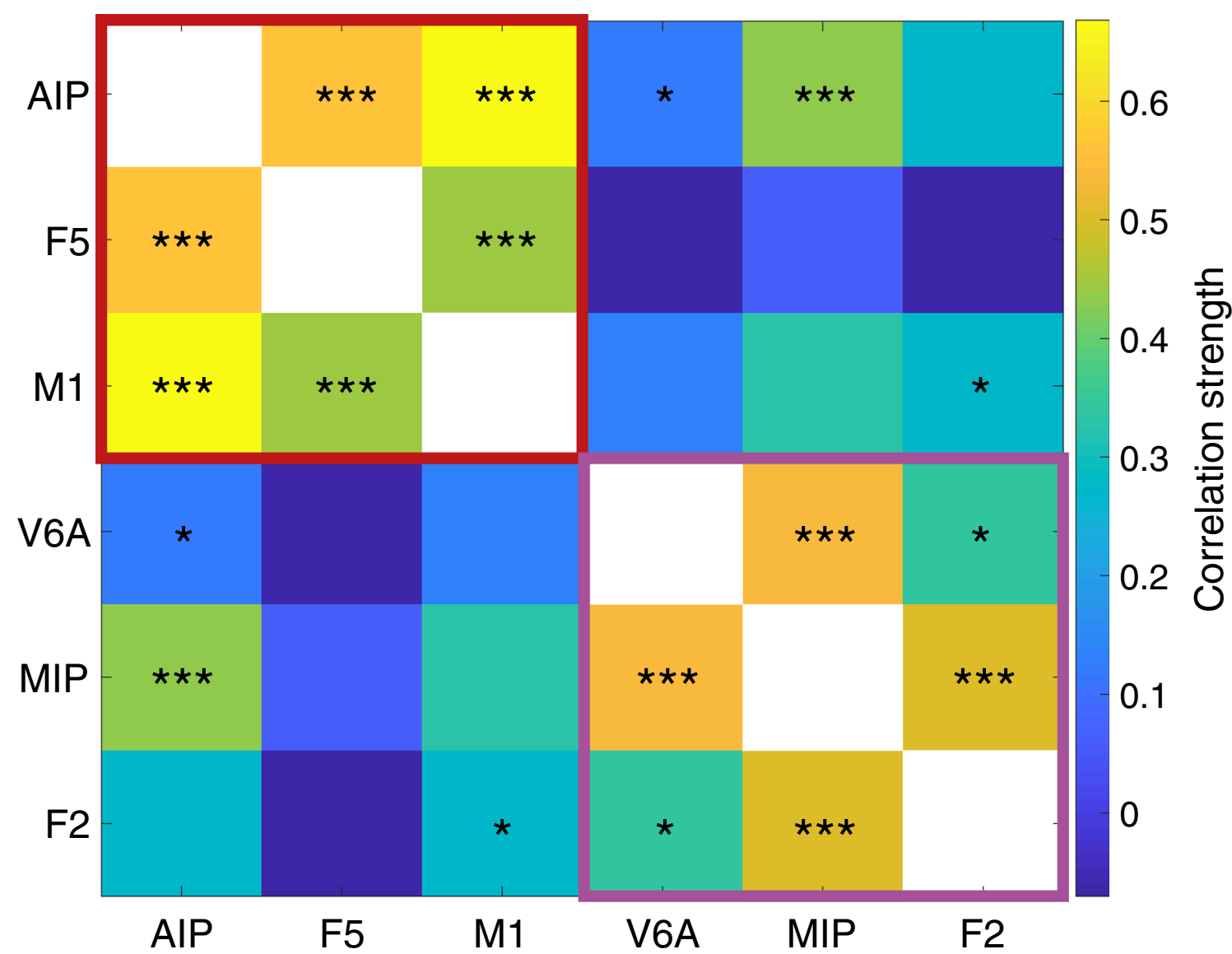

Figure 6. Between seed correlation matrix. Color bar showing the mean correlation between areas over all analyzed animals. Colored frames show the two significant $(\mathrm{p}=0.0049)$ clusters as assessed by optimal modularity ${ }^{38}$. Significance of the correlation between each pair of seed region was tested with a one-sided $t$ test; ${ }^{*} \mathrm{p}<0.05$ Bonferroni corrected, ${ }^{* *} \mathrm{p}<0.01$ Bonferroni corrected. Plotted with Matlab Ver. 9.5.0.1067069 (R2018b) Update 4 (https://de.mathworks.com/products/matlab.html).

Functional connectivity map of dorsal premotor cortex (area F2). By placing seeds in dorsal premotor cortex (area F2), we found widespread correlation on the dorsal part of the brain (Fig. 4, bottom panels), covering the superior parietal lobule, the supramarginal gyrus, and dorsal parts of the post- and precentral gyrus. Along the midline and dorsal parts of the cortex we found correlations in the precuneus, cingulate, and lingual gyrus. In the posterior hemisphere, significant correlations appeared along the annectant gyrus and the angular gyrus dorsal to the beginning of the inferior occipital sulcus. In the temporal lobe, correlations were present in the upper third of the middle and superior temporal gyrus, whereas in the frontal lobe strong correlations were present in the superior frontal gyrus and around the principal sulcus. Furthermore, we found strong correlations along the insular cortex.

Subcortically, we saw significant correlations in the superior colliculus, putamen, claustrum, around the pedunculopontine tegmental and cuneiform nuclei, and in the body and parts of the head of the caudate nucleus (Fig. 5, bottom panels). Surprisingly, we found correlations in the right medial globus pallidus and much stronger correlations in the left compared to the right oral pulvinar.

Although all correlation maps had a high degree of symmetry across both hemispheres, most correlations were more widespread on the left, ipsilateral side. In contrast, the correlation map of F2 was more widespread on the contralateral hemisphere. In particular, we found stronger correlations in the right (contralateral) cuneus, the right intraparietal sulcus, in the posterior part of the superior temporal sulcus, and the insular cortex. Correlations in the middle frontal and inferior frontal gyri, however, showed stronger correlation in the left hemisphere.

Functional connectivity between seed maps. In addition to individual seed maps, we also calculated the average correlation between the time series of each pair of seed regions across runs (Fig. 6). Strongest significant correlations were found between pairs of seed regions of the same network: AIP/M1 $1_{\text {hand }}$ and AIP/F5 of the grasp-related dorso-ventral network, followed by V6A/MIP and MIP/F2 of the reach-related dorso-medial network. Most correlations between seed region pairs from different networks (AIP/F2, F5/F2, F5/V6A and $\mathrm{M}_{\text {hand }} / \mathrm{V} 6 \mathrm{~A}$ ) were not significant when tested using a one-sample t-test with Bonferroni correction ( $\mathrm{p}$-value: 0.05), with the exception of MIP/AIP, AIP/V6A, and $\mathrm{F} 2 / \mathrm{M}_{\text {hand }}$. In particular, the correlation between AIP and 


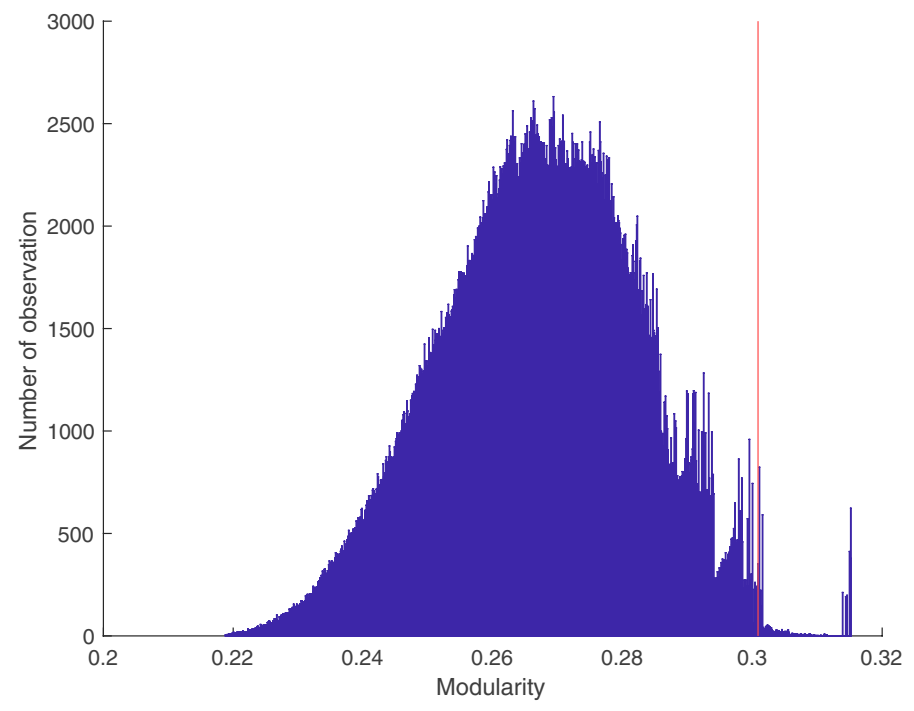

Figure 7. Null distribution of the modularity index of the connectivity matrix. Distribution of the modularity index of 1,000,000 randomly shuffled connectivity matrixes. The red line indicates the modularity index of the original connectivity matrix (0.301). Simulation and plot produced with Matlab Ver. 9.5.0.1067069 (R2018b) Update 4 (https://de.mathworks.com/products/matlab.html).

MIP was noteworthy, since it was also significant at a higher threshold of 0.01 , in contrast to all other correlations across the two designated networks.

These findings strongly suggest that the six seed regions belong to two different cortical networks, one containing AIP, $\mathrm{M}_{\text {hand }}$ and F5 and a second one including MIP, F2 and V6A. To further test this hypothesis, we computed the optimal modularity of the correlation matrix ${ }^{38}$ using the Brain Connectivity Toolbox ${ }^{47}$. As hypothesized, we found a separation into two networks with a modularity index of 0.301 , which was significantly larger than indices produced from surrogate data presuming only a single network $(\mathrm{p}=0.0056$; matrix shuffling over 1,000,000 random iterations; average modularity index: 0.267, see Fig. 7).

Evidence for two separate functional networks. The similarity between the correlation maps of AIP, $\mathrm{M}_{\text {hand }}$ and F5 became apparent at first glance (Fig. 2). Figure 8 (middle panels) displays the shared functional correlations between the three dorso-ventral seed areas AIP, $\mathrm{M1}_{\text {hand }}$, and F5. Areas that appear in the shared dorso-ventral correlation map include the precuneus, the anterior part of the annectant gyrus, the supramarginal, post- and precentral gyrus, and the bilateral intraparietal sulcus (Fig. 8, middle panels). The posterior cingulate gyrus, medial part of the superior frontal gyrus, and the right area 9/46 were also visible in all three maps. In terms of subcortical structures, the putamen and the claustrum showed up in all grasp-related correlation maps (Fig. 9, middle panels).

In the reach-related regions, areas F2, V6A and MIP shared similar patterns in their correlation maps (Fig. 4). The combined correlation map of areas F2, V6A and MIP included large parts of the occipital cortex, starting with the lingual gyrus, predominantly but not exclusively on the right side, the cuneus and precuneus, and the posterior cingulate gyrus (Fig. 8, top panels). We also found large overlaps in the angular gyrus, supramarginal gyrus, superior frontal gyrus and the superior frontal lobule. Along the pre- and postcentral gyrus, the overlap extended laterally from the midline to the end of the intraparietal sulcus. Smaller overlaps were also present along the dorsal aspects of the middle temporal gyrus, the insula, and the principal sulcus. Noteworthy is also area 46, which showed up strongly in both maps. Subcortically, all three correlation maps included the caudate nucleus, putamen, and the claustrum (Fig. 9, top panels).

Distinct and overlapping areas between the dorso-medial and dorso-ventral functional connectivity maps. Finally, we performed a conjunction analysis across both networks by combining the shared correlation map of AIP, M1 $1_{\text {hand }}$ and F5 for the grasp-related dorso-ventral network and the shared correlation map of V6A, F2 and MIP for the reach-related dorso-medial network. The correlation maps for AIP, M1hand, and F5 (dorso-ventral seeds) were clearly distinct from the correlation maps for V6A, F2, and MIP (dorso-medial seeds), yet showed similar correlation patterns with each other, and vice versa for the dorsomedial seeds. Thus, we refer to the combined correlation maps of AIP, $\mathrm{M} 1_{\text {hand }}$, and $\mathrm{F} 5$ as the dorso-ventral network and the combined map of V6A, F2 and MIP as the dorso-medial network (Fig. 8).

When comparing the dorso-medial and dorso-ventral networks, some anatomical structures showed correlations only in one of the two networks while others showed correlations in both (Fig. 8). Although the dorsoventral network was smaller overall than the dorso-medial one, the lateral part of the precentral and postcentral gyrus was significantly connected only to the grasp-related dorso-ventral network. Similarly, also the inferior ramus of the arcuate sulcus, large parts of the insula, and the anterior part of the claustrum were exclusively correlated with the dorso-ventral network. 

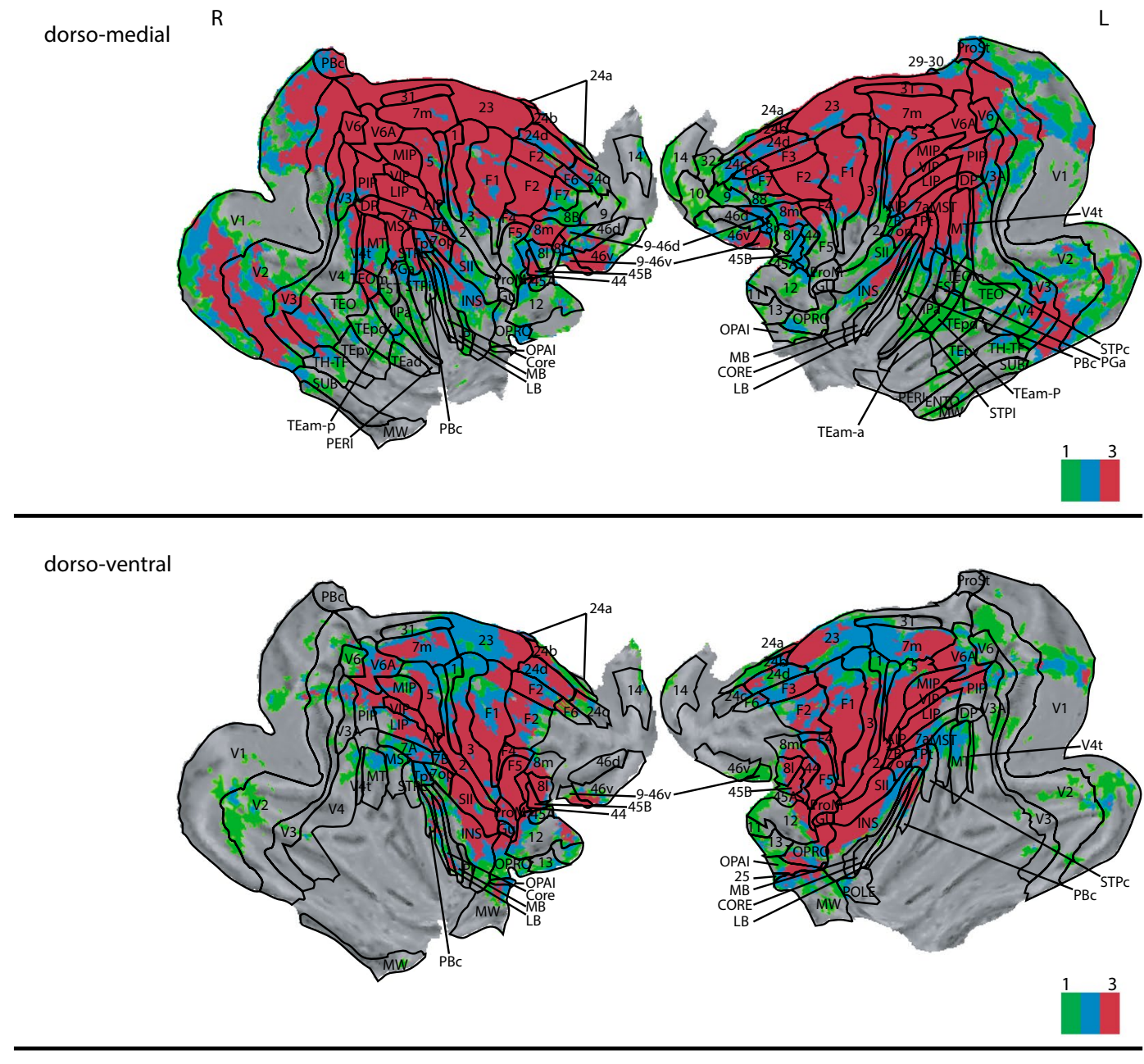

comparison

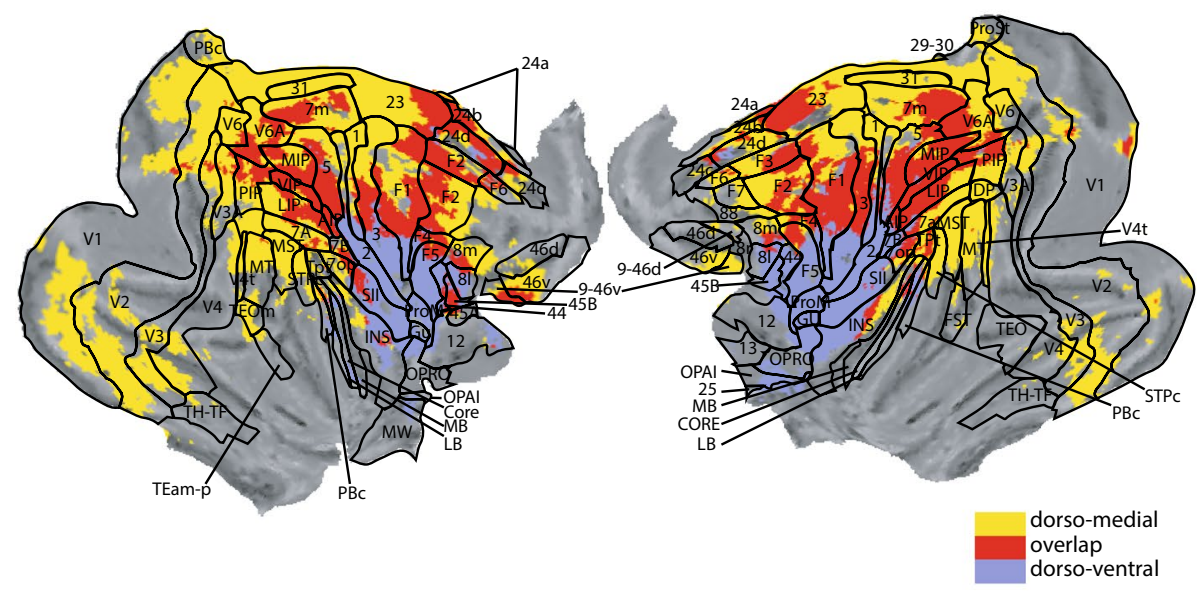

Figure 8. Overlap of the correlation maps of the dorso-medial (top), dorso-ventral (middle), and both networks together (bottom). Overlap was created by projecting the significant voxels of each seed of the respective networks (V6A, MIP and F2 for the dorso-medial and AIP, F5 and M1 $1_{\text {hand }}$ for the dorso-ventral) onto the brain. In the top and middle panel color indicates how often a voxel is significant in the three maps (0-3 times). Voxels that are significant in all three maps of their respective network are considered to show the spatial extend of the network. These voxels are compared in the bottom figure. Here color indicates whether a voxel belongs to the dorso-medial (yellow), dorso-ventral (blue) or both networks (red). All surface renderings (flat maps) were done with CARET v5.65 (https://brainvis.wustl.edu/wiki/index.php/Caret:About) ${ }^{88}$. Cortical area labeling and borders according to ${ }^{43}$. 

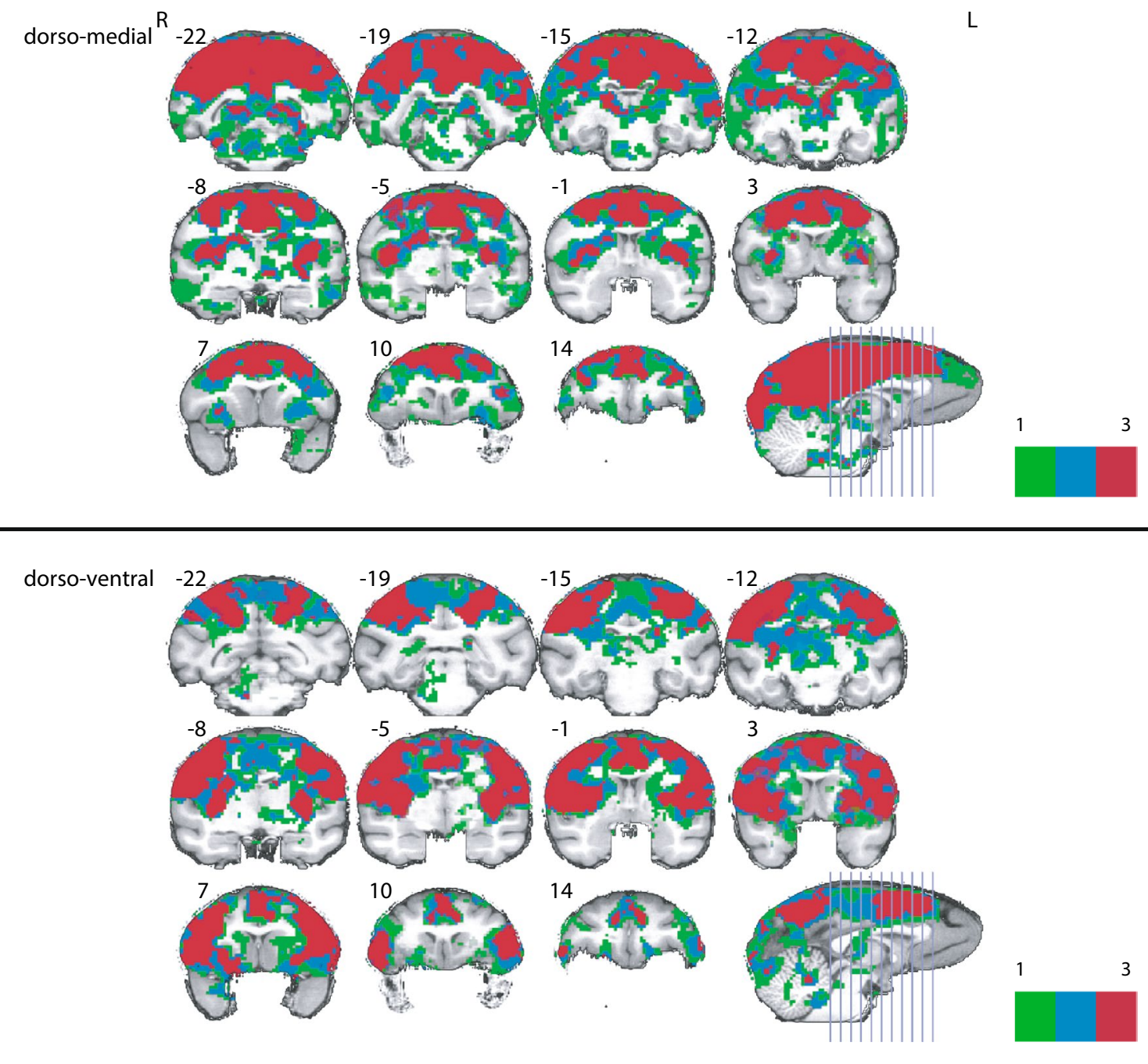

comparison

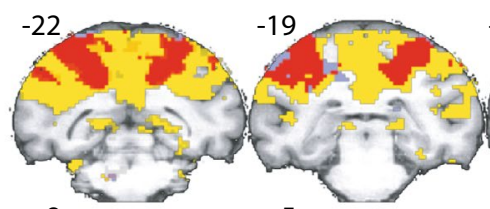

$-15$
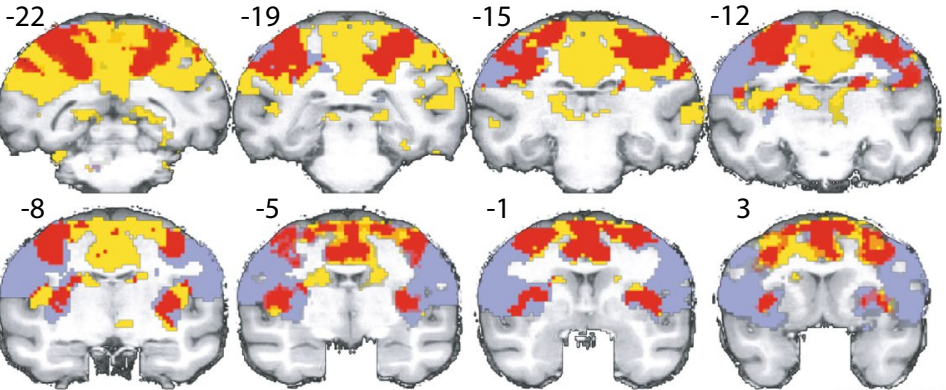

3
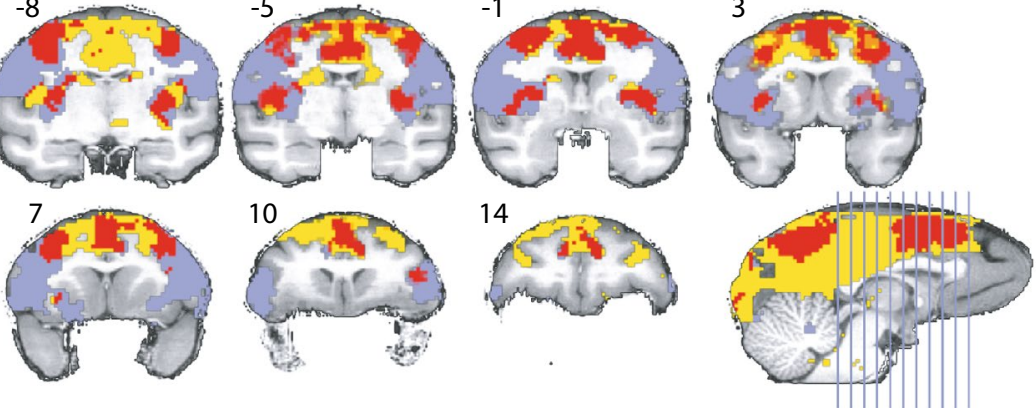

dorso-medial
overlap dorso-ventral

Figure 9. Overlap of the correlation maps of the dorso-medial network seeds (top), the dorso-ventral network seeds (middle) and between both networks (bottom) in the subcortical brain structures. Please note, for ease of comparison, the same color coding is used as in Fig. 8. In the top and middle panel color indicates how often a voxel is significant in the three maps (0-3 times). Bottom panel whether a voxel belongs to the dorso-medial (yellow), dorso-ventral (blue) or both networks (red). Y coordinates of the sections are given relative to the F99 reference frame ${ }^{45}$. Sections rendered with MRIcoGL ver. 1.2.20181114+(https://www.mccauslandcenter.sc.edu/ mricrogl/). 
In contrast, the cuneus, posterior cingulate gyrus, dorsal part of superior temporal sulcus, left inferior frontal gyrus, and the medial pre- and postcentral gyrus were prominently correlated only with the dorso-medial network. In subcortical regions, the head of the caudate nucleus, the right substantia nigra, and the area around the pulvinar nuclei were exclusively correlated to the dorso-medial network (Fig. 9).

However, a number of areas shared functional connectivity across both networks. These included the precuneus, the superior frontal gyrus, the intraparietal sulcus, and the precentral gyrus medial to the spur of the arcuate sulcus. We also observed large overlap along the intraparietal sulcus, central sulcus, insula and cingulate gyrus. In subcortical regions, the putamen, parts of the internal capsule, and the claustrum shared functional connectivity with both networks.

Together, the conjunction analysis revealed that the dorso-medial and dorso-ventral streams are separated into two distinct functional networks, albeit with some shared functional connectivity across areas in both networks.

\section{Discussion}

Using resting-state fMRI of 10 lightly anesthetized macaque monkeys, we obtained connectivity maps from six key cortical areas in the dorso-ventral and dorso-medial network. Individual correlation maps for the seeds AIP, F5, and the hand area of M1 were combined to form the whole-brain correlation map for the dorso-ventral network, while the correlation maps of the seeds V6A, MIP, and F2 were combined to define the dorso-medial network.

Some brain areas were functionally connected to only one of those networks. They represented sensory inputs and cortical functions related to a specific action (i.e., either reaching or grasping). For example, the dorso-ventral network included somatosensory areas in and around the insular cortex, the precentral opercula $\left(\mathrm{PrCO}^{51}\right.$, which includes parts of areas ProM, SII, GU, 2 and 3 in the parcellation of ${ }^{43}$ ), gustatory cortex, and the ventral premotor cortex related to hand grasping. In contrast, the dorso-medial network included higher visual areas and areas relevant for arm reaching. Some brain areas, like MIP and AIP, were part of both networks, indicating shared functionality.

In the between-seed correlation, the strong correlation between AIP and MIP is surprising. Although an anatomical connectivity between MIP and AIP has been reported ${ }^{12}$, it was very weak. However, we observed the strongest connections between both networks with high significance between AIP and MIP.

For the dorso-ventral network, we identified the ventral premotor cortex, including areas F4 and F5, and $\mathrm{PrCO}$ as exclusively correlated with the grasp-related dorso-ventral network. Areas F4 and F5 represent sensory information and complex motor control signals relevant for planning and execution of hand grasping movements. For example, Hepp-Reymond et al. found in both areas neurons that encode grip force ${ }^{52}$, which is highly relevant for fine object manipulation. Furthermore, pharmacological inactivation of area F5 led to specific grasp deficits, but no reach deficits ${ }^{16}$, hence demonstrating an essential role for hand grasping.

The dorso-ventral network is functionally connected with areas involved in feeding behavior, like the PrCO and the primary gustatory cortex ${ }^{53}$. Especially the correlation with gustatory cortex is interesting. It has been shown by tracer studies that the gustatory cortex is connected to primary motor and sensory areas ${ }^{54}$, but a connection with F5 or AIP, to our knowledge, has not been reported. PrCO is monosynaptically connected to F4 and $\mathrm{F} 5^{5}$ and has been associated with mechanical and gustatory stimulation of the tongue ${ }^{55}$. Similarly, we found area PF in the inferior parietal lobule to be exclusively correlated with the dorso-ventral network; PF represents mostly orofacial somatosensory as well as biting and feeding responses ${ }^{56}$. These functional connections are well in line with the behavioral relevance of hand grasping for feeding.

Other areas that showed exclusive correlation with the dorso-ventral network were areas 45 and 7 , the ventral part of areas 2 and F1, and the granular and dysgranular insula. Most of these areas are involved in somatosensory processing, e.g. as seen in area $2^{57}$, in line with a notion that a successful grasp requires somatosensory input about the grasp object to tailor hand shape and grip force ${ }^{58}$.

Regions in premotor and sensorimotor cortex that were exclusively correlated with the reach-related dorsomedial network have been previously shown to encode mainly hindlimb movements $s^{59-61}$. This could be explained by the fact that arm reaching often also involves the extension of the trunk and hindlimbs to reach a far-away target, which requires the coordination of arms and hindlimbs.

In parietal cortex, most of area V6A was exclusively connected to the dorso-medial network and only small parts were functionally connected with both networks. Area V6A has been extensively investigated with respect to its anatomical connectivity ${ }^{7,13,62}$ and electrophysiological selectivity for reaching as well as grasping behavior $23,33,34,36,63$. However, the presence of selective neural activity does not imply a causal influence, as outlined in the introduction. A selective lesion of V6A produced a reach-to-grasp deficit in macaque monkeys with a strong component of incorrect wrist rotation ${ }^{64}$. However, the animals where still able to close their hand in a functional grasp when the target matched the abnormal wrist orientation. This leaves an open question as to whether the observed reach-to-grasp deficits can be attributed to an uncertainty of the reach process and the wrist orientation, or whether they reflect a true grasp deficit (i.e., a deficit in shaping the hand).

Finally, visual areas V1-V4 also show exclusive functional connectivity with the dorso-medial network. In V1 and V2, functional connectivity is focused mainly on the area that represents peripheral vision ${ }^{65}$, a property that was also true for V6A, whose connectivity to peripheral visual areas has been demonstrated with both $\operatorname{tracer}^{7}$ and neurophysiological studies ${ }^{66}$, as well as its encoding of stimulus position in craniotopic coordinates ${ }^{66}$. Reaching requires the position of an object in space, which is predominantly acquired by vision.

Reaching and grasping are closely related motor actions that are often executed together. Tight connectivity between both networks that control these actions is therefore necessary to facilitate a precise and meaningful interaction with the outside world. 
We found shared functional connectivity in areas along the intraparietal sulcus (LIP, VIP, V6A, MIP and PIP) that have been shown to provide visual or somatosensory input to premotor areas, e.g., for eye coordination ${ }^{67-70}$ In the supramarginal gyrus, part of the areas PFG and PGop ${ }^{71}$ were significantly correlated with both networks. PFG showed connections to F4 and $\mathrm{F}^{72}$ and has been observed to encode complex somatotopic input not restricted to the hand and arm area $^{73}$. In an extensive mapping of the parietal lobule, Rozzi et al. found sensory and motor neurons similarly distributed to our findings ${ }^{56}$. For example, area PFG contained somatosensory neurons with response fields mainly from the hand and arm, visually responsive neurons to presented objects, and grasp-related motor, peri-personal and mirror neurons. Cortical planning of reaching and grasping actions might require rather similar sensory input, given that both actions need careful sensory coordination.

We also found substantial overlap in the precuneus, whose involvement in reaching and grasping is currently unclear. Although the precuneus has been described as a hub of the default mode network both in humans ${ }^{74}$ and monkeys ${ }^{75}$, the overlap between networks did not appear to correspond with the default mode network. However, tracer studies have shown that the precuneus projects both to the dorsal premotor area F5 and V6 $\mathrm{A}^{13,76,77}$. Therefore, it is likely that the precuneus correlation is functional rather than caused by the default mode network. Since the precuneus is activated when both hands are coordinated to perform a complex task ${ }^{78}$, we hypothesize that the precuneus plays a role for the coordination of reaching and grasping networks.

Finally, we found shared functional connectivity between networks with the frontal eye field (FEF, area $8 \mathrm{~m}$ ), which is central for the control of gaze directions ${ }^{79}$ and the integration of sensory information from the dorsal and ventral visual stream ${ }^{80}$. The FEF likely plays an important role for the sensory guidance of arm and hand movements and furthermore for hand-eye coordination.

In conclusion, this study showed that both the dorso-medial and dorso-ventral stream clearly separation at the functional level. The grasp-related dorso-ventral stream is connected more strongly with somatosensory areas, while the reach-related dorso-medial stream has strong connections to visual areas. This separation may be due to arm reaching relying on visual input, while grasping relies more strongly on somatosensory input. This has also been observed in patients who lost their sense of touch; such patients were able to point towards a specific position in space, but had unrecoverable deficits in grasping and manipulating visible objects ${ }^{81}$.

Importantly, we found significant correlations between major areas of both networks, demonstrating that the dorso-medial and dorso-ventral networks are strongly interconnected. Reach and grasp movements are often performed in a coordinated fashion towards a common action goal (e.g., reaching for a branch and climbing the branch or grasping and eating a food item). Connections between these reaching and grasping networks are therefore essential for the spatio-temporal integration and coordination of these actions.

One possible explanation for this interconnection may be adopted from the predictive coding model proposed for motor actions ${ }^{82-85}$. In this model, an efference copy of the actions from an upstream brain area is used by downstream areas to efficiently interpret its input, but this efference copy does not causally influence the actions of the downstream area. This may explain why neural activity in the reach-related dorso-medial stream is correlated with grasping and vice versa.

Finally, our study highlights additional areas that might be involved in the neuronal processing of reaching and grasping actions. For example, the role of the dorsolateral prefrontal cortex (area 9/46) for reaching and grasping has not been well studied but shows extensive functional connectivity with the dorso-medial network. Furthermore, the precentral opercular area and the insular cortex were strongly connected to the dorso-ventral network, as previously demonstrated in tracer studies ${ }^{86,87}$, but without a clear role for the coordination of reaching and grasping. Further electrophysiological studies of these areas may provide new insights into the sensorimotor integration necessary for the successful coordination of reaching and grasping actions.

Received: 7 May 2020; Accepted: 7 October 2020

Published online: 29 October 2020

\section{References}

1. Jeannerod, M. Intersegmental coordination during reaching at natural visual objects. Atten. Perform. IX, 153-168 (1981)

2. Binkofski, F. \& Buxbaum, L. J. Two action systems in the human brain. Brain Lang. 127, 222-229 (2013).

3. Jeannerod, M. Visuomotor channels: Their integration in goal-directed prehension. Hum. Mov. Sci. 18, 201-218 (1999).

4. Jeannerod, M., Arbib, M. A., Rizzolatti, G. \& Sakata, H. Grasping objects: The cortical mechanisms of visuomotor transformation. Trends Neurosci. 18, 314-320 (1995).

5. Matelli, M., Camarda, R., Glickstein, M. \& Rizzolatti, G. Afferent and efferent projections of the inferior area 6 in the macaque monkey. J. Comp. Neurol. 251, 281-298 (1986).

6. Tanné, J., Boussaoud, D., Boyer-Zeller, N. \& Rouiller, E. M. Direct visual pathways for reaching movements in the macaque monkey. NeuroReport 7, 267 (1995).

7. Shipp, S., Blanton, M. \& Zeki, S. A visuo-somatomotor pathway through superior parietal cortex in the macaque monkey: Cortical connections of areas V6 and V6A. Eur. J. Neurosci. 10, 3171-3193 (1998)

8. Tanné-Gariépy, J., Boussaoud, D. \& Rouiller, E. M. Projections of the claustrum to the primary motor, premotor, and prefrontal cortices in the macaque monkey. J. Comp. Neurol. 454, 140-157 (2002).

9. Borra, E. et al. Cortical connections of the macaque anterior intraparietal (AIP) area. Cereb. Cortex 18, 1094-1111 (2008).

10. Gamberini, M. et al. Cortical connections of the visuomotor parietooccipital area V6Ad of the macaque monkey. J. Comp. Neurol. 513, 622-642 (2009).

11. Bakola, S., Gamberini, M., Passarelli, L., Fattori, P. \& Galletti, C. Cortical connections of parietal field PEc in the macaque: Linking vision and somatic sensation for the control of limb action. Cereb. Cortex 20, 2592-2604 (2010).

12. Bakola, S. et al. Cortical afferents and myeloarchitecture distinguish the medial intraparietal area (MIP) from neighboring subdivisions of the macaque cortex. eNeuro 4, 20 (2017).

13. Passarelli, L. et al. Cortical connections of area V6Av in the macaque: A visual-input node to the eye/hand coordination system. J. Neurosci. 31, 1790-1801 (2011). 
14. Gamberini, M., Passarelli, L., Fattori, P. \& Galletti, C. Structural connectivity and functional properties of the macaque superior parietal lobule. Brain Struct. Funct. https://doi.org/10.1007/s00429-019-01976-9 (2019).

15. Gallese, V., Murata, A., Kaseda, M., Niki, N. \& Sakata, H. Deficit of hand preshaping after muscimol injection in monkey parietal cortex. NeuroReport 5, 1525 (1994).

16. Fogassi, L. et al. Cortical mechanism for the visual guidance of hand grasping movements in the monkeyA reversible inactivation study. Brain 124, 571-586 (2001).

17. Tunik, E., Frey, S. H. \& Grafton, S. T. Virtual lesions of the anterior intraparietal area disrupt goal-dependent on-line adjustments of grasp. Nat. Neurosci. 8, 505 (2005).

18. Rice, N. J., Tunik, E. \& Grafton, S. T. The anterior intraparietal sulcus mediates grasp execution, independent of requirement to update: New insights from transcranial magnetic stimulation. J. Neurosci. 26, 8176-8182 (2006).

19. Hwang, E. J., Hauschild, M., Wilke, M. \& Andersen, R. A. Inactivation of the parietal reach region causes optic ataxia, impairing reaches but not saccades. Neuron 76, 1021-1029 (2012).

20. Cavina-Pratesi, C., Connolly, J. D. \& Milner, A. D. Optic ataxia as a model to investigate the role of the posterior parietal cortex in visually guided action: Evidence from studies of patient MH. Front. Hum. Neurosci. 7, 20 (2013).

21. Rizzolatti, G. et al. Functional organization of inferior area 6 in the macaque monkey. Exp. Brain Res. 71, 491-507 (1988)

22. Batista, A. P., Buneo, C. A., Snyder, L. H. \& Andersen, R. A. Reach plans in eye-centered coordinates. Science 285, 257-260 (1999).

23. Fattori, P., Gamberini, M., Kutz, D. F. \& Galletti, C. 'Arm-reaching' neurons in the parietal area V6A of the macaque monkey. Eur. J. Neurosci. 13, 2309-2313 (2001).

24. Sakata, H., Taira, M., Murata, A. \& Mine, S. Neural mechanisms of visual guidance of hand action in the parietal cortex of the monkey. Cereb. Cortex 5, 429-438 (1995).

25. Fogassi, L. et al. Parietal lobe: From action organization to intention understanding. Science 308, 662-667 (2005).

26. Baumann, M. A., Fluet, M.-C. \& Scherberger, H. Context-specific grasp movement representation in the macaque anterior intraparietal area. J. Neurosci. 29, 6436-6448 (2009).

27. Galletti, C., Kutz, D. F., Gamberini, M., Breveglieri, R. \& Fattori, P. Role of the medial parieto-occipital cortex in the control of reaching and grasping movements. Exp. Brain Res. 153, 158-170 (2003).

28. Fattori, P., Breveglieri, R., Amoroso, K. \& Galletti, C. Evidence for both reaching and grasping activity in the medial parieto-occipital cortex of the macaque. Eur. J. Neurosci. 20, 2457-2466 (2004).

29. Raos, V., Umiltá, M.-A., Gallese, V. \& Fogassi, L. Functional properties of grasping-related neurons in the dorsal premotor area F2 of the macaque monkey. J. Neurophysiol. 92, 1990-2002 (2004).

30. Fattori, P. et al. Hand orientation during reach-to-grasp movements modulates neuronal activity in the medial posterior parietal area V6A. J. Neurosci. 29, 1928-1936 (2009).

31. Kakei, S., Hoffman, D. S. \& Strick, P. L. Direction of action is represented in the ventral premotor cortex. Nat. Neurosci. 4, 1020 (2001).

32. Stark, E., Asher, I. \& Abeles, M. Encoding of reach and grasp by single neurons in premotor cortex is independent of recording site. J. Neurophysiol. 97, 3351-3364 (2007).

33. Fattori, P. et al. The dorsomedial pathway is not just for reaching: Grasping neurons in the medial parieto-occipital cortex of the macaque monkey. J. Neurosci. 30, 342-349 (2010).

34. Gamberini, M., Galletti, C., Bosco, A., Breveglieri, R. \& Fattori, P. Is the medial posterior parietal area V6A a single functional area?. J. Neurosci. 31, 5145-5157 (2011).

35. Lehmann, S. J. \& Scherberger, H. Reach and gaze representations in macaque parietal and premotor grasp areas. J. Neurosci. 33, 7038-7049 (2013)

36. Breveglieri, R., Bosco, A., Galletti, C., Passarelli, L. \& Fattori, P. Neural activity in the medial parietal area V6A while grasping with or without visual feedback. Sci. Rep. 6, 20 (2016).

37. Birn, R. M. The behavioral significance of spontaneous fluctuations in brain activity. Neuron 56, 8-9 (2007).

38. Newman, M. E. J. Modularity and community structure in networks. Proc. Natl. Acad. Sci. USA 103, 8577-8582 (2006).

39. Gilbert, K. M., Gati, J. S., Barker, K., Everling, S. \& Menon, R. S. Optimized parallel transmit and receive radiofrequency coil for ultrahigh-field MRI of monkeys. NeuroImage 125, 153-161 (2016).

40. Shattuck, D. W. \& Leahy, R. M. BrainSuite: An automated cortical surface identification tool. Med. Image Anal. 6, 129-142 (2002).

41. Van Essen, D. C. Surface-based approaches to spatial localization and registration in primate cerebral cortex. NeuroImage 23(Supplement 1), S97-S107 (2004).

42. Ghahremani, M., Hutchison, R. M., Menon, R. S. \& Everling, S. Frontoparietal functional connectivity in the common marmoset. Cereb. Cortex https://doi.org/10.1093/cercor/bhw198 (2016).

43. Markov, N. T. et al. A weighted and directed interareal connectivity matrix for macaque cerebral cortex. Cereb. Cortex 24, 17-36 (2014).

44. Saleem, K. S. \& Logothetis, N. K. A Combined MRI and Histology Atlas of the Rhesus Monkey Brain in Stereotaxic Coordinates (Academic Press, New York, 2012).

45. Van Essen, D. C. Surface-based atlases of cerebellar cortex in the human, macaque, and mouse. Ann. N. Y. Acad. Sci. 978, 468-479 (2002).

46. Schaffelhofer, S., Agudelo-Toro, A. \& Scherberger, H. Decoding a wide range of hand configurations from macaque motor, premotor, and parietal cortices. J. Neurosci. 35, 1068-1081 (2015).

47. Rubinov, M. \& Sporns, O. Complex network measures of brain connectivity: Uses and interpretations. NeuroImage 52, $1059-1069$ (2010).

48. Davare, M., Kraskov, A., Rothwell, J. C. \& Lemon, R. N. Interactions between areas of the cortical grasping network. Curr. Opin. Neurobiol. 21, 565-570 (2011).

49. Simonyan, K. \& Fuertinger, S. Speech networks at rest and in action: Interactions between functional brain networks controlling speech production. J. Neurophysiol. 113, 2967-2978 (2015).

50. Gregoriou, G. G., Borra, E., Matelli, M. \& Luppino, G. Architectonic organization of the inferior parietal convexity of the macaque monkey. J. Comp. Neurol. 496, 422-451 (2006)

51. Roberts, T. S. \& Akert, K. Insular and opercular cortex and its thalamic projection in Macaca mulatta. Schweiz. Arch. Neurol. Neurochir. Psychiatr. Arch. Suisses Neurol. Neurochir. Psychiatr. 92, 1-43 (1963).

52. Hepp-Reymond, M.-C., Hüsler, E. J., Maier, M. A. \& Qi, H.-X. Force-related neuronal activity in two regions of the primate ventral premotor cortex. Can. J. Physiol. Pharmacol. 72, 571-579 (1994).

53. Ogawa, H. Gustatory cortex of primates: anatomy and physiology. Neurosci. Res. 20, 1-13 (1994)

54. Cipolloni, P. B. \& Pandya, D. N. Cortical connections of the frontoparietal opercular areas in the Rhesus monkey. J. Comp. Neurol. 403, 431-458 (1999).

55. Ito, S. \& Ogawa, H. Neural activities in the fronto-opercular cortex of macaque monkeys during tasting and mastication. Jpn. J. Physiol. 44, 141-156 (1994).

56. Rozzi, S., Ferrari, P. F., Bonini, L., Rizzolatti, G. \& Fogassi, L. Functional organization of inferior parietal lobule convexity in the macaque monkey: Electrophysiological characterization of motor, sensory and mirror responses and their correlation with cytoarchitectonic areas. Eur. J. Neurosci. 28, 1569-1588 (2008). 
57. Pons, T. P., Garraghty, P. E., Cusick, C. G. \& Kaas, J. H. The somatotopic organization of area 2 in macaque monkeys. J. Comp. Neurol. 241, 445-466 (1985).

58. Intveld, R. W., Dann, B., Michaels, J. A. \& Scherberger, H. Neural coding of intended and executed grasp force in macaque areas AIP, F5, and M1. Sci. Rep. 8, 1-16 (2018).

59. Marshall, W. H., Woolsey, C. N. \& Bard, P. Cortical representation of tactile sensibility as indicated by cortical potentials. Science 85, 388-390 (1937).

60. Kaas, J. H., Nelson, R. J., Sur, M., Lin, C. S. \& Merzenich, M. M. Multiple representations of the body within the primary somatosensory cortex of primates. Science 204, 521-523 (1979).

61. Godschalk, M., Mitz, A. R., van Duin, B. \& vander Burga H. ,. Somatotopy of monkey premotor cortex examined with microstimulation. Neurosci. Res. 23, 269-279 (1995).

62. Galletti, C. et al. The cortical connections of area V6: An occipito-parietal network processing visual information. Eur. J. Neurosci. 13, 1572-1588 (2001).

63. Fattori, P., Kutz, D. F., Breveglieri, R., Marzocchi, N. \& Galletti, C. Spatial tuning of reaching activity in the medial parieto-occipital cortex (area V6A) of macaque monkey. Eur. J. Neurosci. 22, 956-972 (2005).

64. Battaglini, P. P. et al. Effects of lesions to area V6A in monkeys. Exp. Brain Res. 144, 419-422 (2002)

65. Daniel, P. M. \& Whitteridge, D. The representation of the visual field on the cerebral cortex in monkeys. J. Physiol. 159, 203-221 (1961)

66. Galletti, C., Fattori, P., Kutz, D. F. \& Gamberini, M. Brain location and visual topography of cortical area V6A in the macaque monkey. Eur. J. Neurosci. 11, 575-582 (1999).

67. Galletti, C., Battaglini, P. P. \& Fattori, P. Parietal neurons encoding spatial locations in craniotopic coordinates. Exp. Brain Res. 96 221-229 (1993).

68. Robinson, C. J. \& Burton, H. Organization of somatosensory receptive fields in cortical areas $7 \mathrm{~b}$, retroinsula, postauditory and granular insula of M. fascicularis. J. Comp. Neurol. 192, 69-92 (1980).

69. Blatt, G. J., Andersen, R. A. \& Stoner, G. R. Visual receptive field organization and cortico-cortical connections of the lateral intraparietal area (area LIP) in the macaque. J. Comp. Neurol. 299, 421-445 (1990).

70. Krubitzer, L., Clarey, J., Tweedale, R., Elston, G. \& Calford, M. A redefinition of somatosensory areas in the lateral sulcus of macaque monkeys. J. Neurosci. 15, 3821-3839 (1995).

71. Luppino, G. \& Rizzolatti, G. The organization of the frontal motor cortex. Physiology 15, 219-224 (2000).

72. Pandya, D. N. \& Seltzer, B. Intrinsic connections and architectonics of posterior parietal cortex in the rhesus monkey. J. Comp. Neurol. 204, 196-210 (1982).

73. Rozzi, S. et al. Cortical Connections Of The Inferior Parietal Cortical Convexity Of The Macaque Monkey. Cereb. Cortex 16 1389-1417 (2006)

74. Robinson, C. J. \& Burton, H. Somatic submodality distribution within the second somatosensory (SII), 7b, retroinsular, postauditory, and granular insular cortical areas of M. fascicularis. J. Comp. Neurol. 192, 93-108 (1980).

75. Fransson, P. \& Marrelec, G. The precuneus/posterior cingulate cortex plays a pivotal role in the default mode network: Evidence from a partial correlation network analysis. NeuroImage 42, 1178-1184 (2008).

76. Mantini, D. et al. Default mode of brain function in monkeys. J. Neurosci. 31, 12954-12962 (2011).

77. Johnson, P. B., Ferraina, S., Bianchi, L. \& Caminiti, R. Cortical networks for visual reaching: Physiological and anatomical organization of frontal and parietal lobe arm regions. Cereb. Cortex 6, 102-119 (1996).

78. Passarelli, L. et al. Uniformity and diversity of cortical projections to precuneate areas in the macaque monkey: What defines area PGm?. Cereb. Cortex 28, 1700-1717 (2018).

79. Wenderoth, N., Debaere, F., Sunaert, S. \& Swinnen, S. P. The role of anterior cingulate cortex and precuneus in the coordination of motor behaviour. Eur. J. Neurosci. 22, 235-246 (2005).

80. Thompson, K. G., Hanes, D. P., Bichot, N. P. \& Schall, J. D. Perceptual and motor processing stages identified in the activity of macaque frontal eye field neurons during visual search. J. Neurophysiol. 76, 4040-4055 (1996).

81. Schall, J. D., Morel, A., King, D. J. \& Bullier, J. Topography of visual cortex connections with frontal eye field in macaque: Convergence and segregation of processing streams. J. Neurosci. 15, 4464-4487 (1995).

82. Cole, J. \& Paillard, J. Living without touch and peripheral information about body position and movement: Studies with deafferented subjects. Body Self 20, 245-266 (1995).

83. Rao, R. P. N. \& Ballard, D. H. Predictive coding in the visual cortex: A functional interpretation of some extra-classical receptive field effects. Nat. Neurosci. 2, 79-87 (1999).

84. Wolpert, D., Ghahramani, Z. \& Jordan, M. An internal model for sensorimotor integration. Science 269, 1880-1882 (1995).

85. Kilner, J. M., Friston, K. J. \& Frith, C. D. Predictive coding: An account of the mirror neuron system. Cogn. Process. 8, 159-166 (2007).

86. Shipp, S., Adams, R. A. \& Friston, K. J. Reflections on agranular architecture: Predictive coding in the motor cortex. Trends Neurosci. 36, 706-716 (2013)

87. Pribram, K. H. \& MacLean, P. D. Neuronographic analysis of medial and basal cerebral cortex: II. Monkey. J. Neurophysiol. 16 324-340 (1953)

88. Showers, M. J. C. \& Lauer, E. W. Somatovisceral motor patterns in the insula. J. Comp. Neurol. 117, 107-115 (1961).

89. Van Essen, D. C. et al. An integrated software suite for surface-based analyses of cerebral cortex. J. Am. Med. Inform. Assoc. 8, 443-459 (2001)

\section{Acknowledgements}

We thank Joseph Gati and Trevor Szekeres for technical support with original MRI data collection. This work was supported by Deutsche Forschungsgemeinschaft (German Research Foundation), SFB 889, project C9 to H.S. and the Canadian Institutes of Health Research Grant FRN148365 to S.E.

\section{Author contributions}

R.S.G. and H.S. conceived the research; S.E. and R.A. contributed data; R.S.G. and R.A. designed the data analysis. R.S.G. performed the data analysis. R.S.G. wrote the manuscript. R.S.G., R.A., S.E., and H.S. revised and approved the final version of the manuscript.

\section{Funding}

Open Access funding enabled and organized by Projekt DEAL.

\section{Competing interests}

The authors declare no competing interests. 


\section{Additional information}

Supplementary information is available for this paper at https://doi.org/10.1038/s41598-020-75219-x.

Correspondence and requests for materials should be addressed to R.S.G. or H.S.

Reprints and permissions information is available at www.nature.com/reprints.

Publisher's note Springer Nature remains neutral with regard to jurisdictional claims in published maps and institutional affiliations.

(c) Open Access This article is licensed under a Creative Commons Attribution 4.0 International License, which permits use, sharing, adaptation, distribution and reproduction in any medium or format, as long as you give appropriate credit to the original author(s) and the source, provide a link to the Creative Commons licence, and indicate if changes were made. The images or other third party material in this article are included in the article's Creative Commons licence, unless indicated otherwise in a credit line to the material. If material is not included in the article's Creative Commons licence and your intended use is not permitted by statutory regulation or exceeds the permitted use, you will need to obtain permission directly from the copyright holder. To view a copy of this licence, visit http://creativecommons.org/licenses/by/4.0/.

(C) The Author(s) 2020 


\subsection{Project II: PriMa: A low-cost, modular, open hardware, and 3D-printed fMRI] manipulandum}

\section{Author contributions}

R.S. Greulich and H. Scherberger conceived the research; R.S. Greulich, T. Hüser, and $M$. Dörge designed and build the hardware; M. Dörge implemented the software; R.S. Greulich and T. Hüser performed the experiments and wrote the manuscript; all authors revised the manuscript.

\subsubsection{General Discussion}

The second project designs a device that allows the examination of grasp and reach in experimental paradigms in the fMRI scanner. We termed this device a manipulandum, since it is meant to be manipulated by the subject. The fMRI environment poses two unique challenges to motor research that had to be considered during the design of said manipulandum.

The first challenge concerns the safety of the subject. All experimental devices introduced to the scanner room have to be fMRl safe. The device cannot contain large pieces of ferromagnetic metals. Smaller parts of non-ferromagnetic metals have to be designed carefully to avoid induction loops. We circumvented this problem by designing the manipulandum completely metal free. The non-metal rule can be relaxed for the control box, as it will be kept outside of the scanner room.

The second challenge was to report the behavior of the subject. The manipulandum needed a way to detect and report the actions of the subject. However, conventional switches are not compatible with the fMRI safety requirements. We therefore designed 3D printable switches that interrupt the light path between two light fibers. This allows us to detect the switch action via a control box.

The control box was housed outside of the scanner and feeds light from highpower LED into the light fibers. The response was detected via a photo transistor, amplified, and thresholded with an adjustable rate. The resulting binary signal was read in real time via a digitization USB-card into the controller software and recorded.

For the basic form of the manipulandum, we settled on two bars in an L-shape. This design would allow two different orientations of the grasp. One would be close to the rest position and the other only reachable with a short reach action. We placed buttons on the manipulandum to detect two different grasps, a two-finger 
precision grip and a whole hand power grip. While the power grip is considered the evolutionary older and more basic behavior, the precision grasp is unique to primates (see section 1.1 on page 1).

We constructed a human and macaque scale variant of the manipulandum. Crossspecies comparison is essential for motor research. Electrophysiological results are almost exclusively available from $\mathrm{NHP}$, fMRI research into motor actions predominately from humans. Direct comparisons between both species implementing the same experiment are rare. Such studies would fall under the transfer type of replication studies (Brendel et al., 2020) and would give strength or show the limitations of our knowledge transfer from NHP to humans.

We kept the design modular to ease maintenance and repair. All buttons were designed as a single 3D printable unit. The main chassis was also 3D printed and provides slots for the button assemblies. This modularity also allows the design to be adjusted for other experiments. Different designs for the main body are easily constructed. We demonstrated this with a 6-button box mock-up in the publication. To enable other researchers to reuse our design, we released all design files under an open hardware license.

The influence of the human manipulandum on the signal-to-noise ratio (SNR) of the MRI recordings were measured with a spherical phantom. The relative change in SNR was under $5 \%$, which we consider to be acceptable.

We tested the human design in a pilot study. One human subject was cued to execute all four possible grasps in a random order. We showed that the head movement parameters were within acceptable limits and that the manipulandum produces no visible artifacts.

The macaque manipulandum was mounted to the $\mathrm{fMRl}$-compatible macaque chair. The animals accepted this set-up very well and were able to execute the grasps during task training.

This work lays the technical foundation for the third project. We have demonstrated that we can record grasping behavior in the MRI scanner. This allows us to look into grasp and reach behavior in humans and awake macaques. The main open questions are if reach and grasp are utilizing separate networks during behavior and how both processes are coordinated in time. The third project implements those research questions utilizing a delayed response paradigm and multi voxel pattern analysis

This manuscript was submitted to Neurolmage on January $28^{\text {th }}, 2021$, is currently under review and included as submitted. 


\title{
PriMa: A low-cost, modular, open hardware, and 3D-printed fMRI manipulandum
}

\author{
R. Stefan Greulich ${ }^{1,2,}$, Timo Hüser ${ }^{1,3,}{ }^{*}$, Matthias Dörge $^{1}$, \\ Hansjörg Scherberger ${ }^{1,2}$ \\ $27^{\text {th }}$ January 2021
}

Corresponding Author: Hansjörg Scherberger

Affiliations:

${ }^{1}$ Deutsches Primatenzentrum GmbH, Kellnerweg 4, 37077 Göttingen, Germany

${ }^{2}$ Faculty of Biology and Psychology, University of Goettingen, Germany

${ }^{3}$ Faculty of Physics, University of Göttingen, Germany

* Authors contributed equally to this work

\section{Highlights}

- fMRI safe 3d-printable manipulanda designed for grasping in humans and NHPs

- Light fiber activated buttons and digitalization hardware is presented

- Safety and function are demonstrated on phantom and human MRI scans

- Possible alternative designs and improvements are discussed

- All design files are released under an open hardware license

Keywords: fMRI, 3D printing, open source, neuroscience, sensorimotor processing 


\section{Abstract}

Motor actions in fMRI settings require specialized hardware to monitor, record, and control the subject's behavior. Commercially available options for such behavior tracking or control are very restricted and costly. We present a novel grasp manipulandum in a modular design, consisting of MRIcompatible, 3D printable buttons and a chassis for mounting. Button presses are detected by the interruption of an optical fiber path, which is digitized by a photodiode and subsequent signal amplification and thresholding. Two feedback devices (manipulanda) are constructed, one for macaques (Macaca mulatta) and one for human use. Both devices have been tested in their specific experimental setting and possible improvements are reported. Design files are shared under an open hardware license.

\section{Graphical Abstract}

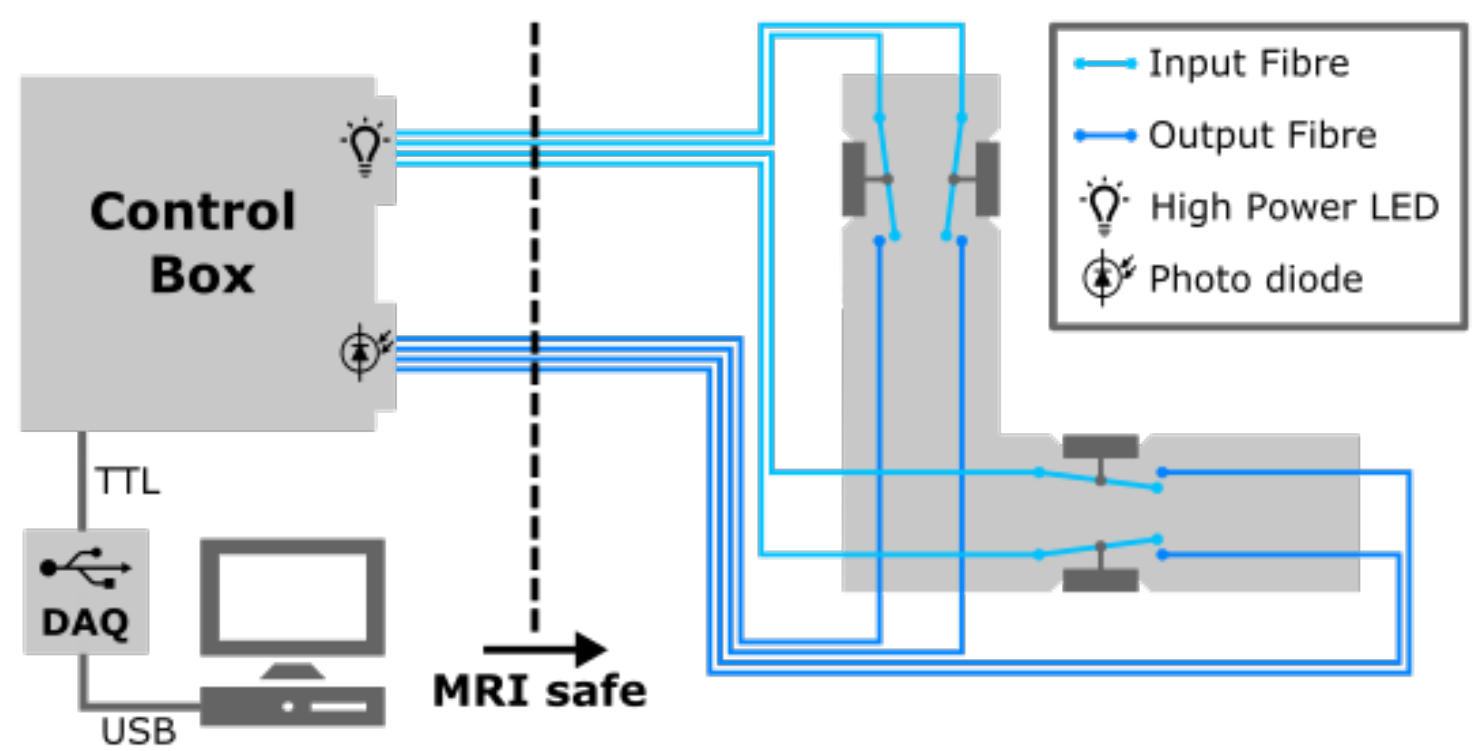

\section{Author contributions}

R. Stefan Greulich: Conceptualization, Methodology, Hardware, Investigation, Writing - Original Draft, Visualization, Project administration

Timo Hüser: Methodology, Hardware, Investigation, Writing - Original Draft, Visualiza- 
tion

Mathias Dörge: Methodology, Hardware, Software

Hansjörg Scherberger: Conceptualization, Writing - Review \& Editing, Supervision, Project administration, Funding acquisition

\section{Funding and conflict of interest statement}

This work was funded by Deutsche Forschungsgemeinschaft (German Research Foundation), SFB 889, project C9. The Funding body had no involvement in the study design, collection, analysis, interpretation of the data, writing nor submission of this work.

Declarations of interest: none 


\section{Introduction}

Motor actions are difficult to investigate in fMRI settings, since even small body movements in the scanner carry an inherent risk for motion artifacts. To circumvent this risk, an often utilized approach is to employ imagined instead of real movements in the task paradigm. However, imagined movement and actually executed movements show only partially overlapping activation patterns in the brain of humans (Lotze et al., 1999; Hanakawa et al., 2003; Nair et al., 2003; Zabicki et al., 2017).

In the past, various experimental setups have been constructed to allow meaningful motor actions during fMRI data acquisition. Examples include the Grasperatus by the Culham lab (Culham et al., 2003) and the pneumatic turntable by the Vanduffel lab (Nelissen and Vanduffel, 2011). However, these manipulanda have been constructed for one particular experimental setup.

Also, only a few commercially available feedback devices exist, ranging from the classic four button bar to gamepads. However, even these standard products cost upwards of $\$ 1000$ each and are not adjustable. There is therefore a strong need for MRI-safe devices that are adjustable to the experimental design while also cheap to implement and maintain.

In the last 40 years, 3D printing has risen from a niche application to a well-established production method (Savini and Savini, 2015). In comparison to traditional manufacturing techniques, 3D printing places fewer constraints on the geometry of the part to be manufactured and allows for low cost production even in very low volume, since the process is highly automated and there is no upfront cost for specialized tooling. In addition, the additive design process provides an unprecedented design freedom that is not available in subtractive manufacturing processes. An intricate internal structure such as bended light fiber guides can be easily encased in a completely closed chassis.

Here, we present a fMRI-compatible manipulandum for hand grasping that is adjustable for human and non-human primate (NHP) experiments. The design is modular and based on 3D printing for manufacturing. We document the design process and demonstrate two concept designs: a human operable version that was tested in fMRI experiments and a NHP version that has been tested during task training with a macaque monkey. Finally, we discuss possible design extensions for future applications. 


\section{Materials \& Methods}

Since the general scientific interest of our lab is about grasp processes in both human and macaques, we identified the following design requirements for the manipulandum:

- modular design to adjust size for human and NHP applications

- metal free to be MRI-compatible

- allow testing of 2 grasp types and 2 hand orientations

- enable measurement of reaction time

- easily maintainable to decrease downtime for repairs and maintenance

- robust against animal interference

To avoid interference with the MRI measurement, all necessary control electronics had to be placed outside of the scanner in the adjacent control room.

We based the design of our manipulandum on an L-shaped form comprising one horizontal and one vertical bar with rectangular cross section $(15 \times 20 \mathrm{~mm}$ for macaques, 30x35 for humans; see figure 3-4), to allow for two different grasps. Each bar can be grasped either with a whole hand power grip, by wrapping the hand around it, or with a precision grip, where two opposing faces are pinched with two fingers.

\subsection{Ethics statement}

All animal care and experiments with the animals were performed in accordance with German and European law and in agreement with the Guidelines for the Care and Use of Mammals in Neuroscience and Behavioral Research (Council, 2003) and the ARRIVE Guidelines (Kilkenny et al., 2010), and were approved by the Animal Welfare Division of the Office for Consumer Protection and Food Safety of the State of Lower Saxony, Germany (permission \#14/1442 and 19/3132).

Subjects for the human evaluation experiment provided written consent, and the experiment was approved by the local Ethics Committee of the Georg Elias Müller Institute of Psychology of the University Göttingen. 


\subsection{Manipulandum for NHPs}

Due to the requirement of measuring reaction times and success rate, a simple bar without any sensors would not suffice. We therefore designed buttons as touch/press sensors that can be 3D-printed as a single building block, in which the compliance of the 3D printed material (here: Nylon 12, printed with the multi jet fusion technique by Shapeways, http://www.shapeways.com/) enabled the elastic button movement (see figure 1 a). This also facilitated ease of repair, since each button can be replaced with minimal disassembly of the full device (see figure 3 a). We designed three different button types: a square-sized smaller button (size 8.5 x $9 \mathrm{~mm}$ ), a rectangular large button (size $7 \times 18 \mathrm{~mm}$ ), and a dual square-sized double button combo (each button 17.6x15x3 mm large). To keep in line with the metal free design criteria, we used optical fibers as signal transmission lines. The buttons were designed to interrupt the light transmission between the input cable and the output cable (see figure $1 \mathrm{a}$ ). This light path interruption was then detected by a photodiode (see 2.4 control box).

a)

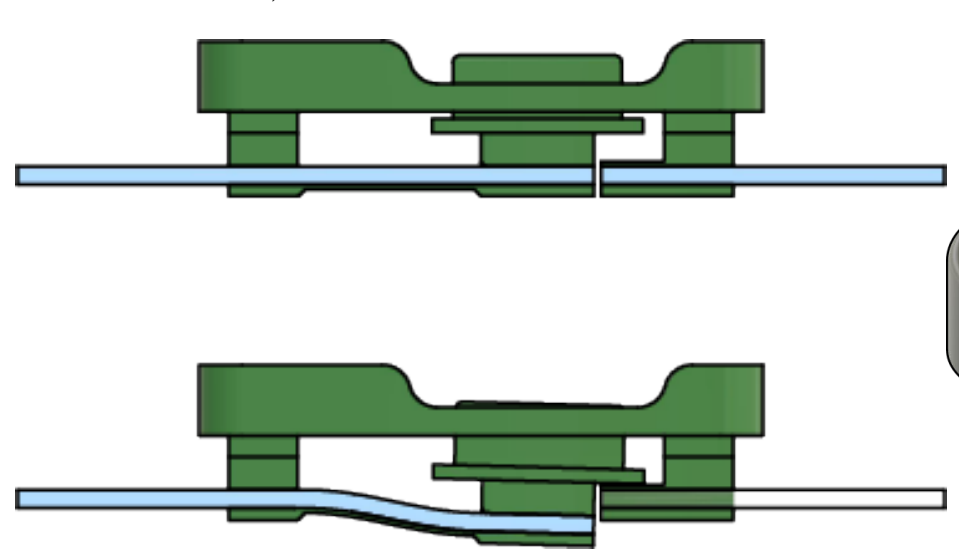

b)

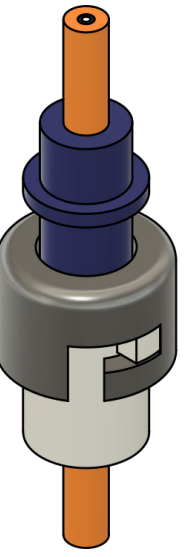

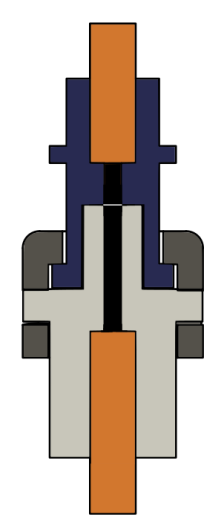

Figure 1: Light fiber button and cable connector. (a) Illustration of the button and its two states: released (top left) and pressed (bottom left). The light path (coming from the left) is blocked when the button is pressed. Right side: Rendering of the optical fiber connector as well as a cross section. (b) The cable connector consists of 3 main parts. The male connector (light gray) connects to the female connector (dark blue) and the whole assembly is locked by the sleeve (dark grey).

The experiment required that the animal is in a defined starting position at the beginning of each trial. Therefore, we added a two button combo as hand rest buttons on a support structure, on level with the horizontal bar and behind the manipulandum from the animal's perspective (see figure $3 \mathrm{~b}$ ). These buttons had to be pressed in order for 


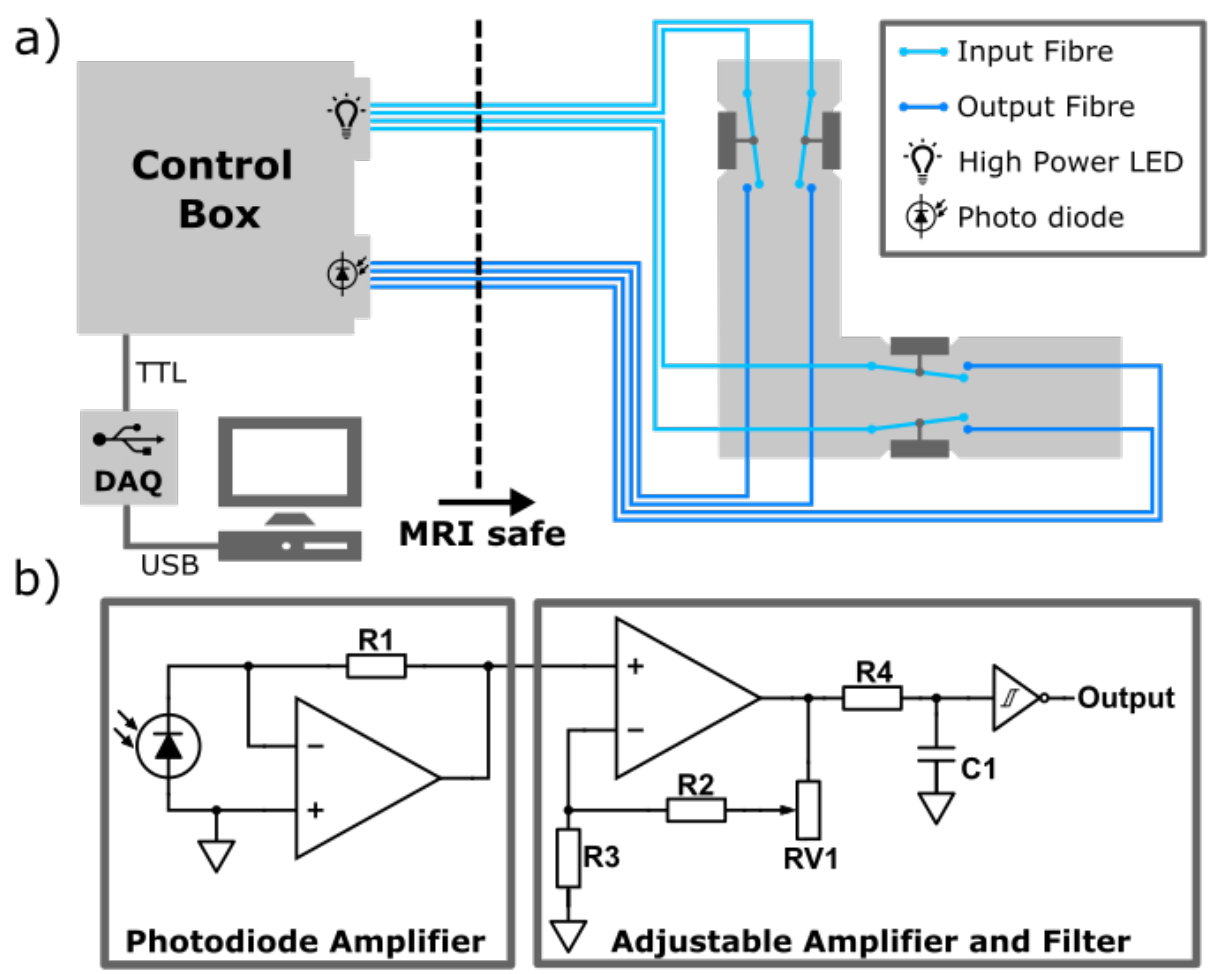

Figure 2: Schematic of the electro-optic circuitry. (a) Input fibers (light blue) feed light to the buttons (dark grey) situated within the manipulandum (light grey). Output fibers (dark blue) report the state of the button back to the control box, where they are converted to electrical (TTL) signals and conveyed to the PC (grey line). (b) Circuit diagram of the receiver end of the control box.. For configuration of resistors and capacitor, see table 1. LED lights are permanently powered.

the trial to start. Instructional cues are displayed on a screen in front of the animal.

To keep the animal from damaging the manipulandum, we ensured that no cables were in reach of the monkey's hand or mouth by routing the optical fibers inside the manipulandum. Due to the number and physical properties of the cables, they needed to be routed along paths with minimal curvature, which would be hard to create using traditional manufacturing techniques. Cables were held in place by clamps that were integrated into the ends of each bar.

We designed a mount to fix the manipulandum on the macaque fMRI compatible chair (Rouge Research Inc., Québec, Canada). This mount allowed free position adjustments of the manipulandum relative to the animal (see figure $3 \mathrm{~b}$ ). Full assembly of the NHP manipulandum and support structure took about 4 hours. 
a)

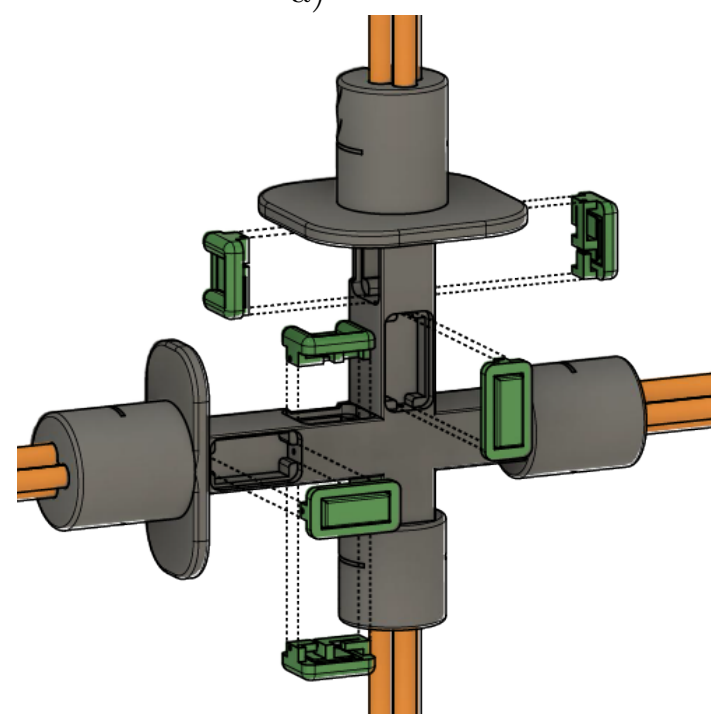

c) b)

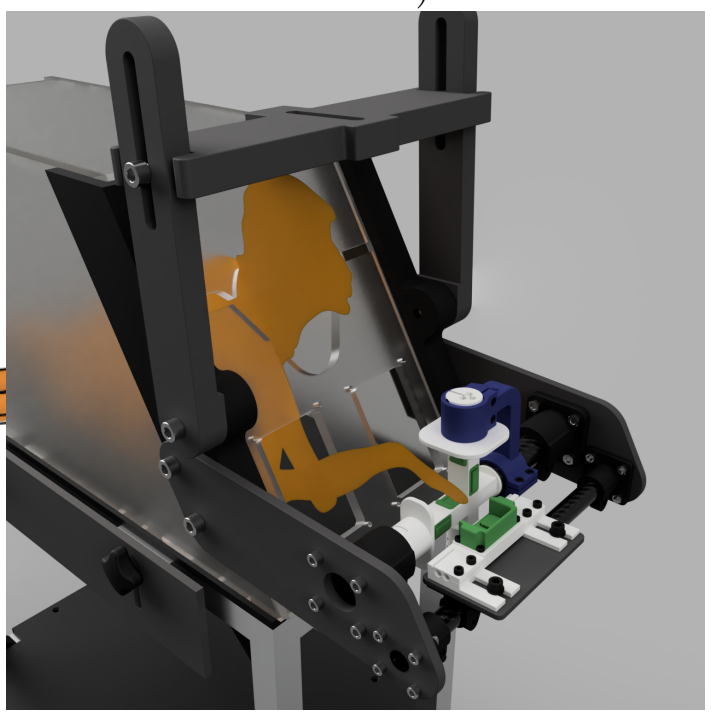

d)

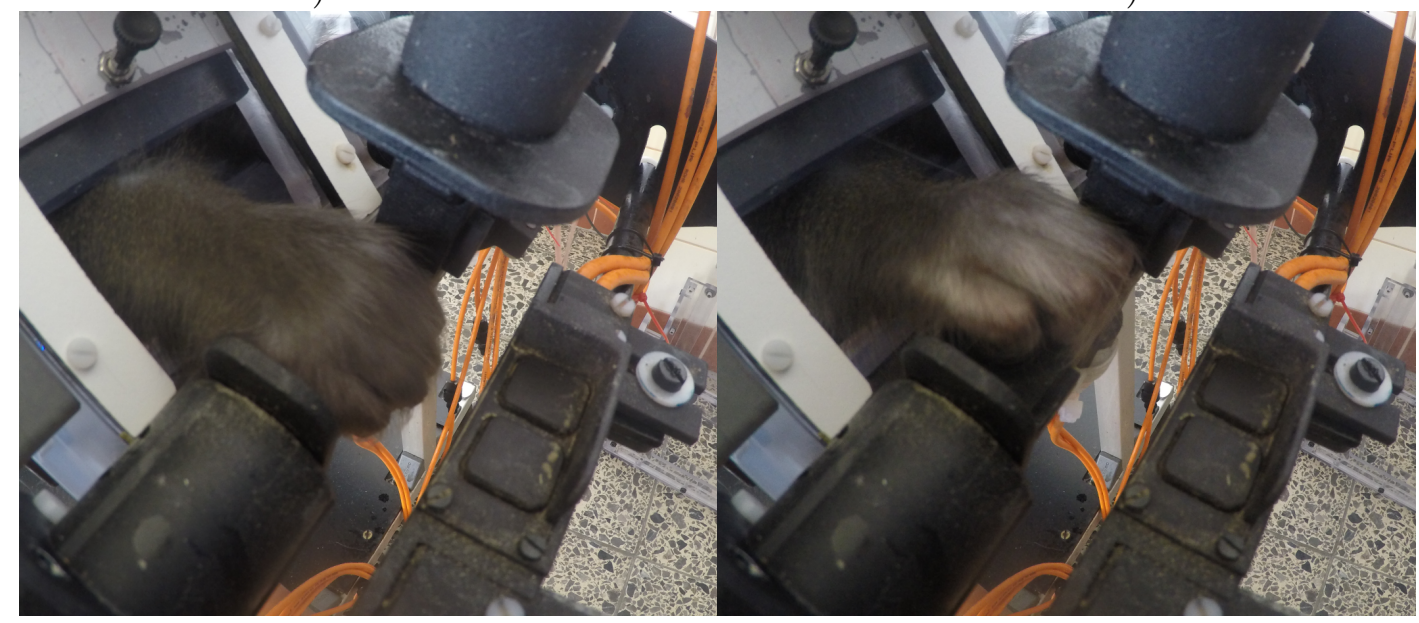

Figure 3: Macaque manipulandum. (a) Explosion diagram showcasing six buttons (green) within the manipulandum body (grey) and the attached optical cables (orange). (b) Manipulandum (white) attached to the primate chair including support structure (black), buttons (green) and a clamp (blue) to give the manipulandum more rigidity against twisting torque by the animal. The finger rest buttons (also green) are to the front right of the manipulandum on a seperate support structure (also white). The actual buttons are obscured by walls integrated into the button assembly to force the animal to use the fingertips instead of the palm. Optical cables are omitted for clarity. (c) Macaque hand performing the power grasp. (d) Macaque hand performing the precision grasp. 


\subsection{Human manipulandum}

The design of the human variant of the manipulandum closely followed the design considerations of the NHP version. Obviously, we could relax the robustness requirements, as human experimental subjects are less prone to destructive behaviour during experimental sessions. In the human manipulandum we also omitted the hand rest buttons, since space constraints were more prevalent and reliable hand rest positions could be achieved simply by auditory instruction and adequate training of the participants.

Furthermore, we adjusted the cross section of the bars $(30 \times 35 \mathrm{~mm})$ and the bar length (104 mm) to be comfortable grasped by humans. Similarly, the size of the buttons was adjusted for human fingers with a size of the square buttons for precision grip of $12 \times 12$ $\mathrm{mm}$ and of the rectangular button for the power grasp of $14 \mathrm{x} 35 \mathrm{~mm}$ (see figure $4 \mathrm{a}$ ). The human manipulandum was mounted on a frame of carbon fiber reinforced plastic (CFRP) using off-the-shelf plastic screws, nuts and plastic connectors. The frame rested on $3 \mathrm{~d}$ printed feet designed to slot into the rail system of the patient bed of our Siemens Prisma $3 \mathrm{~T}$ scanner (see figure $4 \mathrm{~b}$ ).

The full assembly of the human manipulandum and support structure took about 4 hours.

\subsection{D printable cable connectors}

We needed optical fiber cables with a length of $5 \mathrm{~m}$ between the control box and the manipulandum. Although an uninterrupted cable to and from the control box and the buttons can be used, a cable-to-cable connector was designed to ease installation and allow easier maintenance of the manipulandum (see figure $1 \mathrm{~b}$ ). We used a BNC connector inspired bayonet mount as the locking mechanism of the connector.

Cutting the cables to length, striping the insulation and mounting the connectors took about 2 hours in total.

\subsection{Control box}

The control box illuminates the input fiber using white high power LEDs and converts changes of light intensity measured at the output fiber to a TTL signal, indicating the state of the button (pressed: high, released: low: see figure 2 a).

This is done by first using a photodiode transimpedance amplifier to convert the light intensity to an analog voltage $V_{\text {init }}$. The initial amplification is set by the value of $R 1$ following $R 1=\frac{V_{\text {init }}}{-I_{\text {Photo }}}$, where $I_{\text {Photo }}$ is the light-intensity dependent current produced 
a)

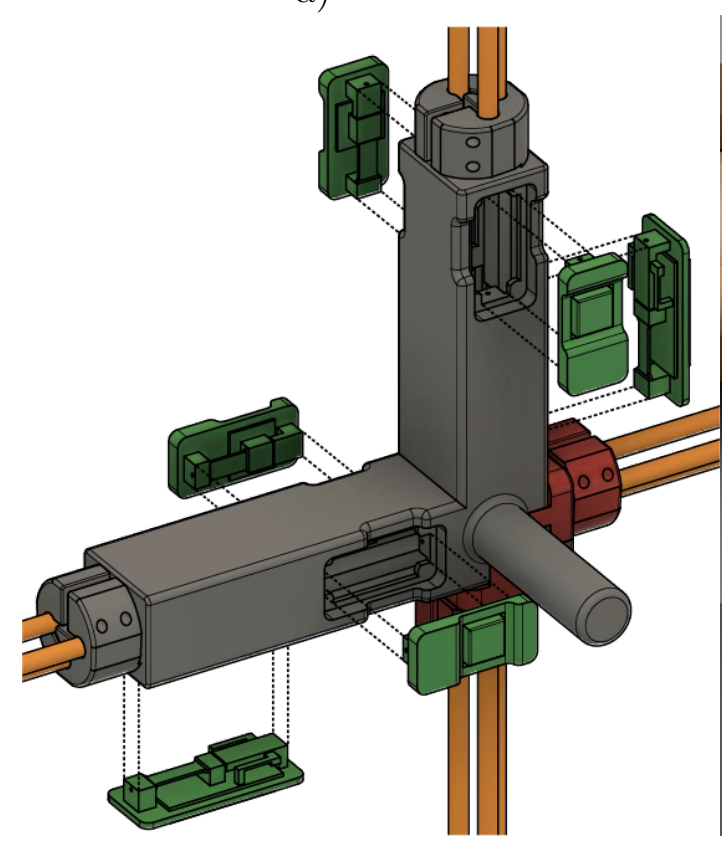

c) b)

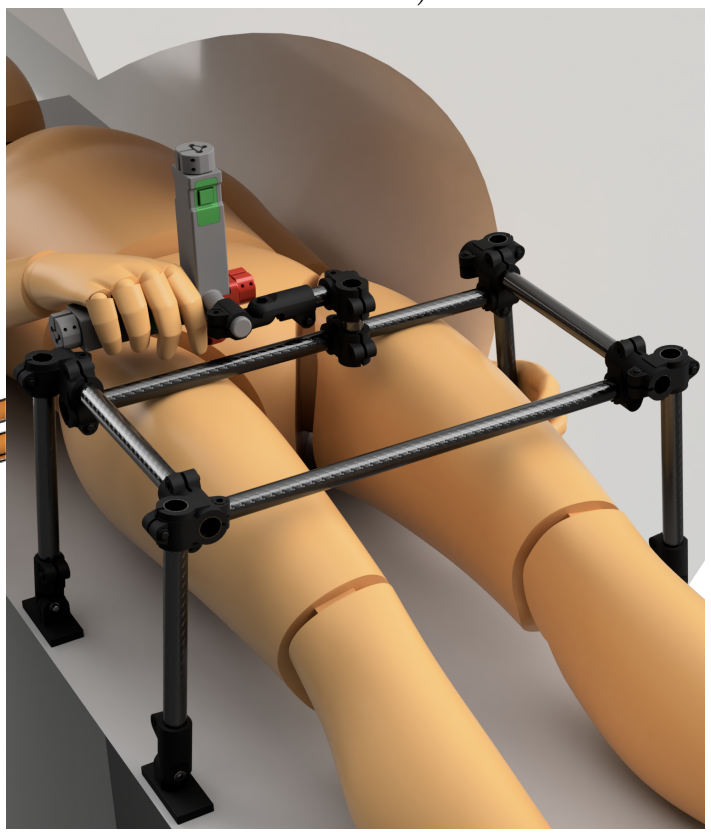

d)

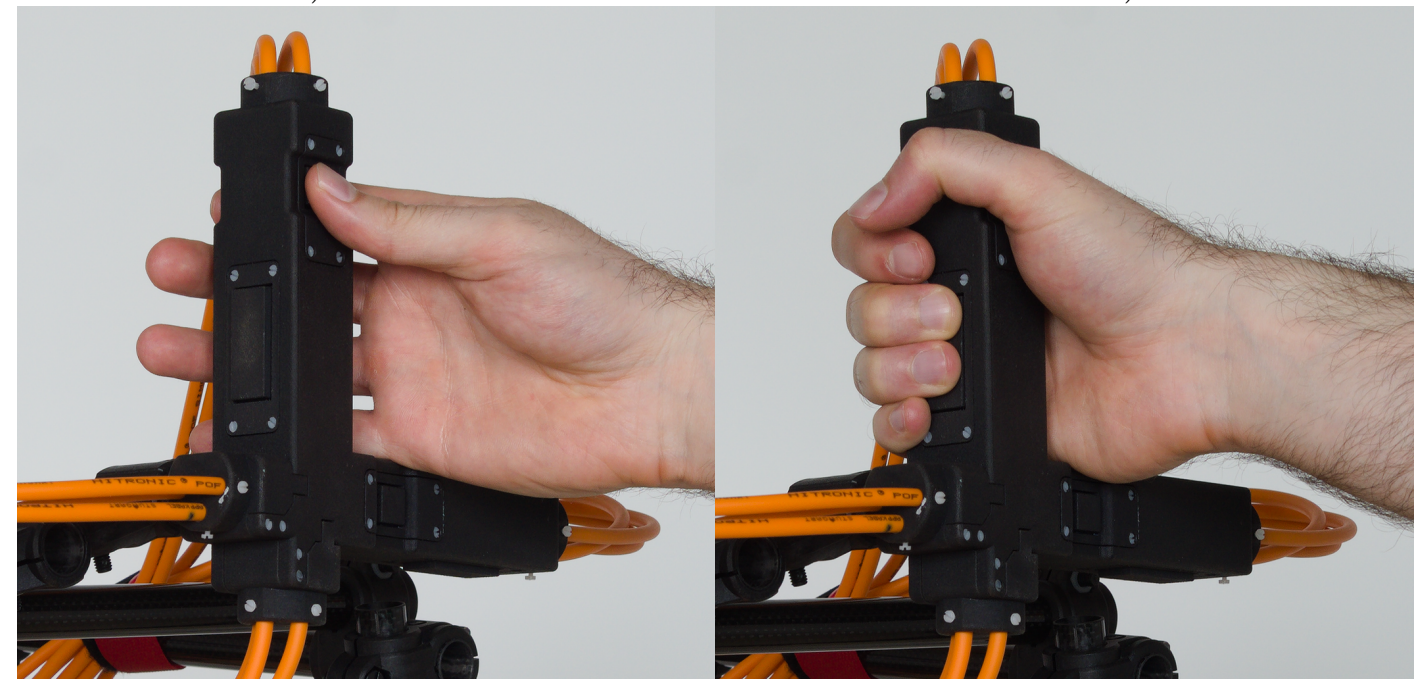

Figure 4: Human manipulandum. (a) Explosion diagram, showcasing six buttons (green) within the manipulandum body (grey) and the attached optical cables (orange). (b) Manipulandum (grey) and support structure (black frame) located on the scanner bed ontop of a subject. Optical cables are not shown for clarity. (c) Demonstration of the vertical precision grip. (d) Demonstration of the vertical power grip. See supplementary materials for images of the complete manipulandum on the mounting frame.

by the photodiode. To allow the circuit to work at different light intensities, the transimpedance amplifier is followed by a variable gain amplifier that can be adjusted via 
a potentiometer. The gain range $\left[G_{l o w}, G_{h i g h}\right]$ available is dependent on the choice of the resistors $R 2, R 3$ and the potentiometer $R V 1$, following $G_{l o w}=1+\frac{R 2}{R 3+R V 1}$ and $G_{\text {high }}=1+\frac{R 2}{R 3}$ (see figure $2 \mathrm{~b}$ for the circuit diagram). To reduce high frequency noise the signal is subsequently low pass filtered by a first order filter with a cutoff frequency of $f_{\text {cutoff }}=\frac{1}{2 \pi R 4 C 1}$. For the values of the resistors and capacitor we picked see table 1 , leading to a cut-off frequency for our filter of $159.15 \mathrm{~Hz}$ and a gain range from 1.044 to 11. Finally a Schmitt trigger is used to invert and threshold the signal, giving the desired digital TTL output. Additionally, the hysteresis of the Schmitt trigger prevents unexpected behavior when the analog voltage crosses the threshold during a button press.

The box can be powered using a single $5 \mathrm{~V}$ power adapter and the output is provided on a DB-25 connector with a parallel port interface compatible pinout. An additional $\mathrm{BNC}$ input connector is available to allow for easy synchronization with an MRI scanner or other recording systems that provide a synchronization signal.

Mounting the components on the PCB and assembly of the control box takes an estimated 10 hours of work.

\subsubsection{Software}

The control box returned a digital signal for each button with 'high' for button pressed and 'low' for button free (0-5 V; TTL logic). A parallel port connection can be used to read the state of the buttons, but support for parallel port cards in modern PCs is dwindling. We decided to use a DB-25 Pin breakout board to record with a simple USB data acquisition device (USB-5901, National Instruments) to read the button states into our LabView control software for experimental control and logging. The data acquisition device measured the states of the input with a sampling rate of $24 \mathrm{MHz}$.

We logged button presses with millisecond precision within LabView (National Instruments). The temporal precision of this recording was dictated by the time delay of the used low pass filter $(0.7 \mathrm{~ms})$ and the precision of the software and operating system, respectively. In our case, we used Windows 7 which is reported to be precise to $1 \mathrm{~ms}$, resulting in a maximal temporal error of less than $2 \mathrm{~ms}$.

\subsection{Evaluation of the SNR influence of the manipulandum}

We first performed a scan on a spherical Phantom (Siemens, Erlangen). Three runs of 2x multiband $\mathrm{T}_{2}^{*}$ weighted EPI (32 slices, 10\% distance factor, $3 \mathrm{~mm}$ slice thickness, 1s 
TR, 37ms TE,192 mm FoV, 3x3 mm voxel size) with each run consisting of 778 volumes were recorded. The first with only the phantom, the second with the human manipulandum completely connected to the control setup and the third with the manipulandum removed, but the control setup connected to the light fiber cables. The last test was done to see if the signal quality was influenced by the inclusion of the control box and fiber cables, which required an open access port between the scanner and control room, reducing the radio interference shielding of the scanner.

The signal to noise ratio (SNR) for a spherical ROI (radius 16 voxels) centered within the phantom recorded with the EPI sequence was calculated by dividing the mean over all voxels with the mean of the standard deviations over all images of a run (Dietrich et al., 2007) for each voxel. From this we calculated the relative difference in SNR between each pair of run as diff $=\frac{\left|S N R_{\text {test }}-S N R_{\text {ref }}\right|}{S N R_{\text {ref }}}$, with run 1 as the reference for runs 2 and 3 , and run 3 as the reference for run 2, to compare all three conditions.

\subsection{Manipulandum evaluation under experimental conditions}

To test the manipulandum in the scanner under experimental conditions, a human subject was placed in the scanner and instructed to perform all four grasps when cued by a visual stimulus (go-cue). Time intervals between go-cues ranged between 6 to 16 seconds. While running this task paradigm, MRI data was recorded on a 3T Siemens Prisma scanner. We recorded 4 runs of $2 \mathrm{x}$ multiband $\mathrm{T}_{2}^{*}$ weighted EPI with the same parameters as above and consisting of 768 volumes, as well as one T1 weighted MPRAGE image (176 slices, $1 \mathrm{~mm}$ slice thickness, 1.9s TR, 3.57ms TE, 256mm FoV, 1x1 mm voxel size). All data was analyzed in SPM 12 (https://www.fil.ion.ucl.ac.uk/spm/) and showed no signs of artifacts (see results).

The NHP version of the manipulandum is currently in use to train two macaque monkeys for an analogous fMRI experiment with a similar grasping paradigm.

\subsection{Data availability statement}

The design files are released under an open hardware licence (CERN-OHL-W) and can be downloaded from:https://github.com/SGatNBL/PriMa. The scans of the phantom are available in the same github repository. The scans of the subject are not released due to data protection concerns. 


\section{Results}

The NHP version of the manipulandum was designed and tested first, and is currently still in use for animal training outside of the scanner. We found that the system worked reliably with no failure during training. However, we discovered a few issues:

1. Using more than one fiber-to-fiber connector per cable led to a strong signal loss, probably due to an imperfect cable to cable alignment within the connector assembly.

2. The buttons turned out to be rather sensitive to residues from dried up reward fluids (juice) dripping into the manipulandum. Adequate placement of the reward system and a drip guard under the reward system are recommended.

Besides these limitations, the manipulandum worked reliably and required only small adjustments of the gain of the amplification circuit. However, if the amplification circuit reached its maximum gain, disassembly and cleaning of the button was required.

The results of the SNR comparison between all three tested conditions are given in table 2 with the measured values given in table 3 .

Overall the relative difference of SNR between all conditions utelizing the phantom was between 0.0040 (phantom \& only control box and light cables connected vs. phantom \& manipulandum fully connected) and 0.0089 (phantom \& only control box and light cables connected vs. phantom only). This demonstrates the low influence of the manipulandum onto the MRI scanner. No visible artifacts were introduced by the presence of the manipulandum.

For the scanning of human subjects, the most prominent source of artifacts is head movement. It is therefore important to properly adjust the position of the manipulandum for the subject, so that all grasps can be executed exclusively with the opening and closing of the fingers and wrist rotations. With those adjustments, the human subject showed no head movement exceeding $0.58 \mathrm{~mm}$ between scans (mean $0.0566 \mathrm{~mm}$, STD $0.0425 \mathrm{~mm}$ over all four runs). This is well below the recommended exclusion threshold of $1 \mathrm{~mm}$ for human fMRI experiments.

We conclude that the manipulandum is safe to use, does only minimally impact the SNR of the recordings and can be grasped by the subject with only minimal head movement artifacts. 


\section{Discussion}

Research of motor control in fMRI settings requires feedback devices that can be easily adjusted to the specific experimental design requirements. Currently available commercial options are restricted to classic button box designs, game controllers, and touch screens. Our design offers modularity and allows adjustment of the chassis for experimental needs. If buttons with different actuating forces are required, a simple design adjustment of the thickness of the compliant part of the mechanism and reprinting is sufficient. See supplemental material for the force response profile of our design. Buttons and casings can easily be implemented in different manipulandum designs. For example, in case a standard 4-button box does not fulfill the experimental needs, a 6-button box could easily be built with the existing button design (see figure 5).

The SNR analysis shows acceptable levels of SNR change (under 5\%) which are probably caused by the requirement to open the shielded access panel to root the light cables through the access ports between scanner and control room.

While working with the human and NHP version of the manipulandum, we noticed a few shortcomings of the design:

1. The buttons are rather sensitive towards dirt and other intrusions, which is a problem especially for the NHP version, where cleanliness cannot be as easily maintained as in human settings.

2. The gain in the control box in the monkey setup needs to be adjusted and the buttons cleaned regularly due to the a both mentioned build up of residues.

3. Our current design needs a bundle of 12 optical fibers (NHP version: 16) to connect the manipulandum with the control box outside of the scanner room. This is rather unwieldy. Using one or several multi-fiber cables instead would be quite advantageous.

4. Finally, it should be noted that the current button design does not provide tactile feedback when operating the buttons.

5. During the testing of the human manipulandum, we noticed that the required depression depth for activation of the large buttons is not uniform across the button, if pressed with just one finger.

The biggest problem we encountered was the movement of the optical fiber cable within its jacket. On first try, we glued the jacket to the cable connector, however 
a)

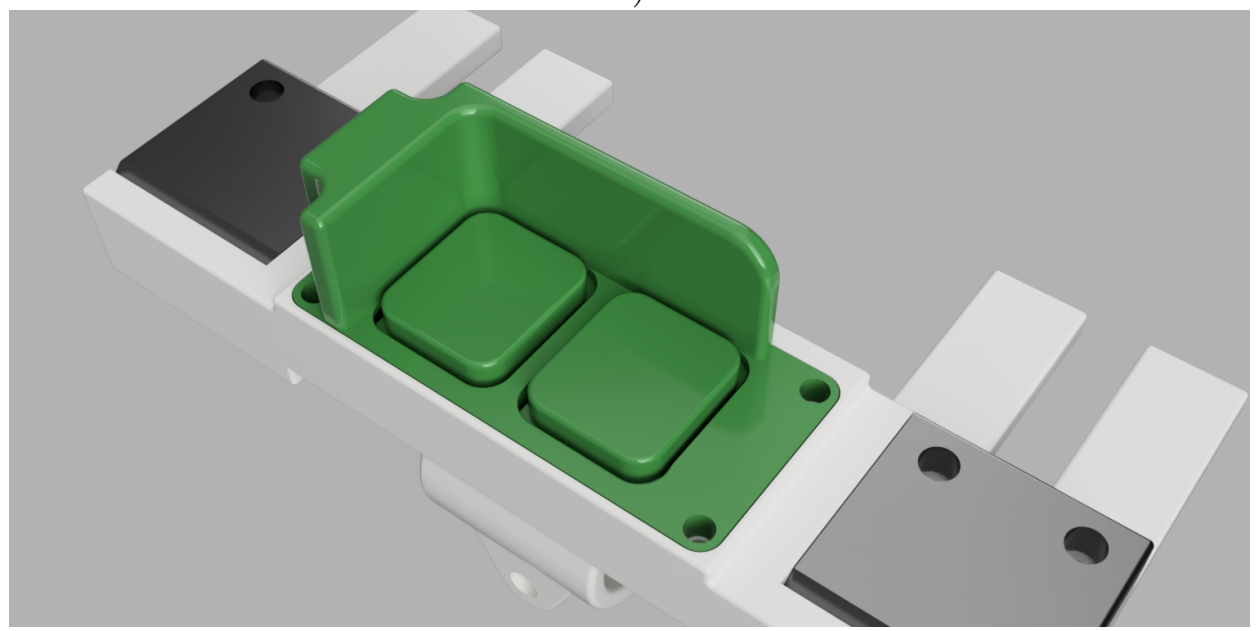

b)

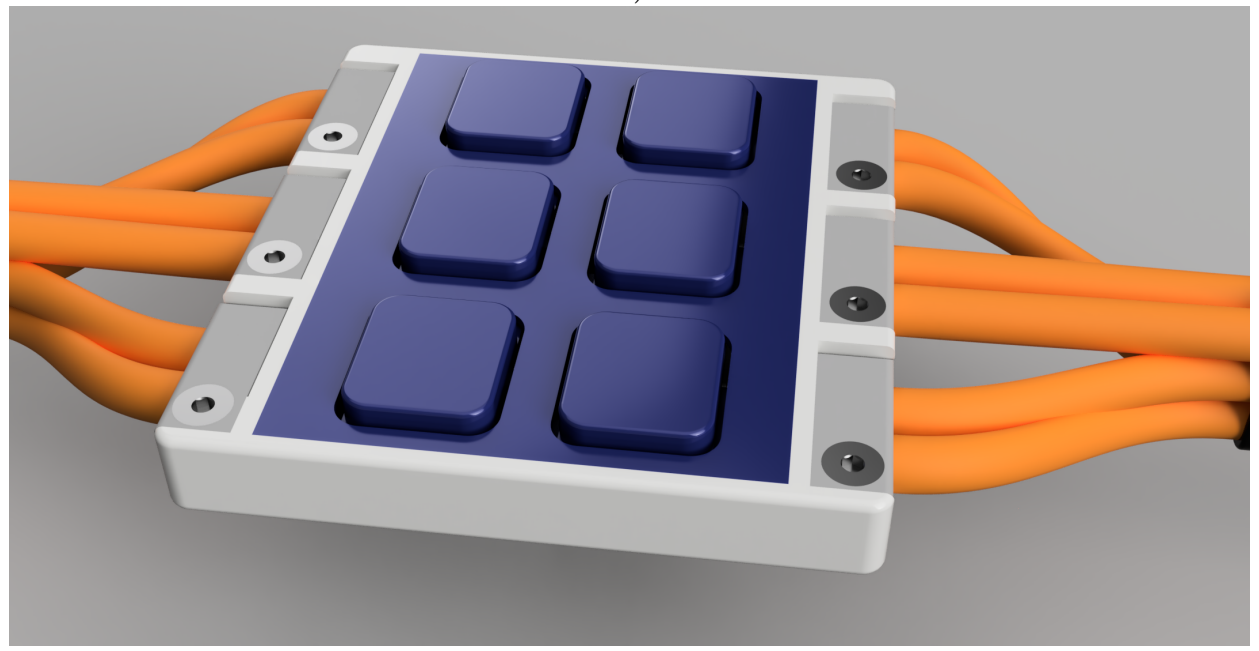

Figure 5: Demonstration of the design steps for a 6 button box. (a) Finger rest button assembly of the macaque setup. The walls of the dual buttons (green) are removed, the cable clamps (black) shortened and the whole assembly copied three times to result in the six button box (b), demonstrating the flexibility of our optical buttons to be adapted for different designs. The design files for this button box are included in the github repository, but it was not build and tested.

this provided an unreliable connection between both cables. We settled on checking this connection first when testing the manipulandum before scanning and did not experienced a failure during scanning yet. The current manipulandum body incorporates a clamp with a grip length of $1.5 \mathrm{~cm}$ to provide strain relief and prohibit movement of the light fibers within the buttons. Our early design of the NHP manipulandum did not provide sufficient fixation of the light fiber and we needed to adjust those 5 times in a time 
span of approx. 30 months. The current design is in use to train 2 animals without any problems occurring in the last 7 months.

The human manipulandum has been used to scan about 30 subjects (approx. 100 presses per button per subject), with the manipulandum failing only once. One button developed a fatigue fracture. The relevant part was reinforced and the manipulandum fixed the same day with replacement parts at hand.

Another possible improvement concerns the power of the light source used. We found that two fiber cable connectors in the whole light path (LED-button-detector) is the maximum possible. If more are included, too much signal is lost. This could be mitigated by utilizing stronger and more focused light sources, such as laser diodes, but this would also require further precautions with respect to laser safety.

\section{Conclusion}

In this work, we presented a fMRI safe design of a graspable manipulandum that is suitable for humans and NHPs. The 3D printable buttons allow for a safe and reliant feedback of the subject's actions. We also demonstrated an adjustment of the design for a six-button box comparable to commercially available feedback devices, which demonstrates the strength of our modular approach. Since design files are openly available for adjustments and $3 \mathrm{~d}$ printing, customization for other fMRI experiments requiring motor responses are straightforward and can be achieved for a very affordable price. 


\section{Tables}

\begin{tabular}{ll}
\hline Component Reference & Value \\
\hline R1 & $5 \mathrm{M} \Omega$ \\
R2 & $220 \mathrm{k} \Omega$ \\
R3 & $22 \mathrm{k} \Omega$ \\
R4 & $10 \mathrm{k} \Omega$ \\
RV1 & $5 \mathrm{M} \Omega$ \\
C1 & $100 \mathrm{nF}$ \\
\hline
\end{tabular}

Table 1: List of the values for all resistors and capacitor in the control box.

\begin{tabular}{lll}
\hline Reference Condition & Test condition & $\begin{array}{l}\text { Relative } \\
\text { difference }\end{array}$ \\
\hline Phantom only & Phantom \& manipulandum & 0.0048 \\
Phantom only & Phantom \& light cables only & 0.0089 \\
Phantom \& light cables only & Phantom \& manipulandum & 0.0040 \\
\hline
\end{tabular}

Table 2: Phantom values updated. Relative difference of the SNR within the phantom between the different conditions (without manipulandum present, with manipulandum present and connected, and manipulandum absent but light cables installed).

\begin{tabular}{llll}
\hline Condition & $\begin{array}{l}\text { mean } \\
\text { intensity }\end{array}$ & mean std. & SNR \\
\hline Phantom only & 973.8979 & 11.3311 & 85.9493 \\
Phantom \& manipulandum & 966.5851 & 11.1918 & 86.3654 \\
Phantum \& light cables only & 962.7614 & 11.1031 & 86.7110 \\
\hline
\end{tabular}

Table 3: Table with measured values added. Measured mean intensity and mean of the std. of the voxels within the ROI for each measured condition and the resulting SNR values. 


\begin{tabular}{|c|c|c|}
\hline Description & Quantity & Price \\
\hline \multicolumn{3}{|c|}{ Controller Box } \\
\hline Printed Circuit Board & 1 & $50,00 €$ \\
\hline Box Connector Mount* & 1 & $29,17 €$ \\
\hline Enclosure & 1 & $6,25 €$ \\
\hline DSUB 25 Pin & 1 & $5,33 €$ \\
\hline BNC Connector & 1 & $1,99 €$ \\
\hline 2.1mm DC Jack & 1 & $3,97 €$ \\
\hline 5V Power Adapter & 1 & $10,99 €$ \\
\hline Terminal Header Part 1 (5 pole) & 1 & $0,35 €$ \\
\hline Terminal Header Part 2 ( 5 pole $)$ & 1 & $0,60 €$ \\
\hline Schrauben M3x5mm & 20 & $0,90 €$ \\
\hline LED High Power White & 10 & $0,95 €$ \\
\hline LED Yellow & 10 & $0,15 €$ \\
\hline LED Green & 1 & $0,15 €$ \\
\hline Photodiode & 10 & $0,74 €$ \\
\hline 74HC14 Schmitt Inverter & 2 & $0,22 €$ \\
\hline TLC272 Operational Amplifier & 10 & $2,10 €$ \\
\hline pChannel Mosfet & 1 & $0,25 €$ \\
\hline Potentiometer $5 \mathrm{M}$ & 10 & $0,28 €$ \\
\hline Zener Diode & 1 & $0,06 €$ \\
\hline \multicolumn{3}{|l|}{ Resistors } \\
\hline $100 \mathrm{k}$ & 10 & $0,04 €$ \\
\hline $3 \mathrm{M}$ & 10 & $0,04 €$ \\
\hline $220 \mathrm{k}$ & 10 & $0,04 €$ \\
\hline $22 \mathrm{k}$ & 10 & $0,04 €$ \\
\hline $75 \mathrm{R}$ & 11 & $0,04 €$ \\
\hline $150 \mathrm{R}$ & 10 & $0,04 €$ \\
\hline $10 \mathrm{k}$ & 1 & $0,04 €$ \\
\hline $1 \mathrm{M}$ & 10 & $0,04 €$ \\
\hline $2 \mathrm{M}$ & 10 & $0,04 €$ \\
\hline $6,8 \mathrm{M}$ & 10 & $0,04 €$ \\
\hline
\end{tabular}

Table 4: Continues on next page 


\begin{tabular}{|c|c|c|}
\hline Description & Quantity & Price \\
\hline \multicolumn{3}{|l|}{ Capacitors } \\
\hline $100 \mathrm{n}$ & 12 & $0,05 €$ \\
\hline $470 \mathrm{n}$ & 2 & $0,24 €$ \\
\hline Total Price Controller Box & & $174,51 €$ \\
\hline \multicolumn{3}{|l|}{ Human SetUp } \\
\hline \multicolumn{3}{|c|}{ Manipulandum } \\
\hline Body* & 1 & $224,14 €$ \\
\hline Button Big* & 2 & $6,90 €$ \\
\hline Button Small* & 4 & $6,90 €$ \\
\hline Cableclamp* & 2 & $11,97 €$ \\
\hline Plastic Screw M4x10mm & 8 & $0,19 €$ \\
\hline \multicolumn{3}{|l|}{ MRI Mount } \\
\hline CFK Pipe $18 \mathrm{~mm}$ & 3 & $20,50 €$ \\
\hline Pipe Connector & 10 & $1,67 €$ \\
\hline Pipe Connector Adjustable Angle & 6 & $2,37 €$ \\
\hline Rail Mounting Feet* & 4 & $23,32 €$ \\
\hline Plastic Screw M6x20mm & 28 & $0,23 €$ \\
\hline Plastic Nut M6 & 28 & $0,17 €$ \\
\hline \multicolumn{3}{|c|}{ Cables and Connectors } \\
\hline Fibre Optic Cable (per meter) & 60 & $1,77 €$ \\
\hline Controller Box Plug Matrix* & 1 & $34,40 €$ \\
\hline Female Cable Connector Matrix* & 1 & $83,21 €$ \\
\hline Male Cable Connector Matrix* & 1 & $57,15 €$ \\
\hline Total Price Manipulandum Human & & $768,86 €$ \\
\hline \multicolumn{3}{|l|}{ Monkey Setup } \\
\hline \multicolumn{3}{|l|}{ Manipulandum } \\
\hline Body* & 1 & $156,23 €$ \\
\hline Button_Big* & 2 & $6,90 €$ \\
\hline Button Small* & 4 & $6,90 €$ \\
\hline Support Body* & 1 & $38,24 €$ \\
\hline
\end{tabular}

Table 4: Continues on next page 


\begin{tabular}{|c|c|c|}
\hline Description & Quantity & Price \\
\hline Support Clamp* & 1 & $8,95 €$ \\
\hline Plastic Screw M4x10mm & 8 & $0,19 €$ \\
\hline \multicolumn{3}{|c|}{ Handrest } \\
\hline Buttons* & 1 & $6,90 €$ \\
\hline Housing Body* & 1 & $21,82 €$ \\
\hline Housing Cable Clamp Left* & 1 & $6,90 €$ \\
\hline Housing Cable Clamp Right* & 1 & $6,90 €$ \\
\hline Plastic Screw M4x10mm & 4 & $0,19 €$ \\
\hline \multicolumn{3}{|c|}{ Cables and Connectors } \\
\hline Fibre Optic Cable (per meter) & 30 & $1,77 €$ \\
\hline Controller Box Plug Matrix* & 1 & $34,40 €$ \\
\hline Total Price Manipulandum Mor & & $377,12 €$ \\
\hline Total Price Human MRI Setup & & $943,37 €$ \\
\hline Total Price Monkey MRI Setup & & $551,63 €$ \\
\hline \multicolumn{3}{|c|}{ Assembly Times } \\
\hline & & Hours \\
\hline Controller Box & & 10 \\
\hline Manipulandum Monkey & & 4 \\
\hline Manipulandum Human & & 4 \\
\hline Cables & & 2 \\
\hline
\end{tabular}

Table 4: List of materials and costs for the human and macaque manipulandum. Part providers are detailed in our github repository (https://github.com/ SGatNBL/PriMa). *3D printed Parts

\section{References}

Council, N. R. (2003). Guidelines for the Care and Use of Mammals in Neuroscience and Behavioral Research. DOI: 10.17226/10732.

Culham, J. C., S. L. Danckert, J. F. X. D. Souza, J. S. Gati, R. S. Menon, and M. A. Goodale (2003). "Visually Guided Grasping Produces fMRI Activation in Dorsal but 
Not Ventral Stream Brain Areas". Exp. Brain Res. 153.2, pp. 180-189. DOI: 10.1007/ s00221-003-1591-5.

Dietrich, O., J. G. Raya, S. B. Reeder, M. F. Reiser, and S. O. Schoenberg (2007). "Measurement of Signal-to-Noise Ratios in MR Images: Influence of Multichannel Coils, Parallel Imaging, and Reconstruction Filters". J. Magn. Reson. Imaging 26.2, pp. 375-385. DOI: 10.1002/jmri.20969.

Hanakawa, T., I. Immisch, K. Toma, M. A. Dimyan, P. Van Gelderen, and M. Hallett (2003). "Functional Properties of Brain Areas Associated With Motor Execution and Imagery". J. Neurophysiol. 89.2, pp. 989-1002. DOI: 10.1152/jn.00132.2002.

Kilkenny, C., W. J. Browne, I. C. Cuthill, M. Emerson, and D. G. Altman (2010). "Improving Bioscience Research Reporting: The ARRIVE Guidelines for Reporting Animal Research". PLOS Biol. 8.6, e1000412. DOI: 10.1371/journal.pbio.1000412. Lotze, M., P. Montoya, M. Erb, E. Hülsmann, H. Flor, U. Klose, N. Birbaumer, and W. Grodd (1999). "Activation of Cortical and Cerebellar Motor Areas during Executed and Imagined Hand Movements: An fMRI Study". J. Cognitive Neurosci. 11.5, pp. 491-501. DOI: 10.1162/089892999563553.

Nair, D. G., K. L. Purcott, A. Fuchs, F. Steinberg, and J. A. S. Kelso (2003). "Cortical and Cerebellar Activity of the Human Brain during Imagined and Executed Unimanual and Bimanual Action Sequences: A Functional MRI Study". Cognitive Brain Res. 15.3, pp. 250-260. DOI: 10.1016/S0926-6410(02)00197-0.

Nelissen, K. and W. Vanduffel (2011). "Grasping-Related Functional Magnetic Resonance Imaging Brain Responses in the Macaque Monkey". J. Neurosci. 31.22, pp. 82208229. DOI: 10.1523/JNEUROSCI . 0623-11.2011.

Savini, A. and G. Savini (2015). "A Short History of 3D Printing, a Technological Revolution Just Started". 2015 ICOHTEC/IEEE International History of High-Technologies and Their Socio-Cultural Contexts Conference (HISTELCON), pp. 1-8. DOI: 10 . 1109/HIStelCON . 2015.7307314.

Zabicki, A., B. de Haas, K. Zentgraf, R. Stark, J. Munzert, and B. Krüger (2017). "Imagined and Executed Actions in the Human Motor System: Testing Neural Similarity Between Execution and Imagery of Actions with a Multivariate Approach". Cereb. Cortex 27.9, pp. 4523-4536. DOI: 10.1093/cercor/bhw257. 


\subsection{Project III: Difference in motor initiation for a predictable or unpredictable go-cue in humans and NHPs utilizing a fMRI grasp paradigm and CVMANOVA}

\section{Author contribution}

R.S. Greulich and H. Scherberger conceived the research; R.S. Greulich collected the data, designed the data analysis, performed the data analysis, and wrote the manuscript.

\subsubsection{General Discussion}

This project aims to bridge the gap between human and NHP motor research. By implementing the same experimental paradigm for both, we can directly compare results across species.

The utilized experimental paradigm allowed us to examine grasping completely without reaching. Additionally, we implemented a second condition with the subject requiring an additional wrist rotation. We postulated that this wrist rotation is a good model for a reach process. The resulting data supports our conclusion by showing significant encoding of the wrist rotation component in the human intrapariatal sulcus.

We also looked into the encoding differences between a predictable and an unpredictable go-cue, allowing us insights into the initiation process for grasp and reachto-grasp motor actions. We found significant encoding in areas related to temporal processing and motor control such as the $\mathrm{SMA}$ and premotor areas.

The human experiment is completed, analyzed, and presented below. However, the macaque experiment is ongoing and is reported as an experiment in progress. Since both are methodologically identical, they are presented together as a standalone chapter with emphasis on the human experiment. We are planning to publish the results of the human experiment soon. 


\title{
Difference in motor initiation for predictable or unpredictable go-cues in humans and NHPs utilizing a fMRI grasp paradigm and CVMANOVA
}

\author{
R. Stefan Greulich ${ }^{1,2}$ and Hansjörg Sherberger ${ }^{1,2}$ \\ 1-Deutsches Primatenzentrum GmbH, Kellnerweg 4, 37077 Göttingen, Germany \\ 2-Faculty of Biology and Psychology, Georg-August-University Göttingen, Germany
}

\begin{abstract}
Reach and grasp are two processes demanding precise control both in space and time. We examined the control in time by examining the neural differences between movement initiation after a predictable or an unpredictable go-cue in humans and NHP. We utilized fMRI, CvMANOVA (Allefeld and Haynes, 2014) and population inference statistics (Allefeld et al., 2016) to identify areas showing significant encoding of our conditions. As an additional research question, we looked into the control in space by comparing the encoding of different grasps and grasp orientation.

We scanned 14 human subjects and found significant encoding of the gocue condition in multiple areas. Most notably are the results in the premotor, primary motor areas, and SMA. The results are in line with previous work in both humans and $\mathrm{NHP}$.

The grasp orientation condition was primarily encoded in the intraparietal sulcus, a region associated with reach processing (Macaluso et al., 2007; Cavina-Pratesi et al., 2010b; Bernier et al., 2017), while grasp is encoded in primary motor cortex. We observe no overlap between reach and grasp encoding areas and the interaction effect shows only a small cluster in the somatosensory cortex, giving support to our hypothesis that wrist rotation to match object orientation is part of the reach process.

A second experiment with two male rhesus macaques and the identical paradigm is proposed. The animals are in the training stage and this experiment is ongoing.
\end{abstract}




\subsubsection{Introduction}

In a groundbreaking work, Haith et al. (2016) showed the independence of movement initiation and planning. The authors forced subjects to move at shorter than normal $\mathrm{RT}$ s and showed that subjects are able to move about $80 \mathrm{~ms}$ faster than their unconstrained RT would imply. The authors concluded that movement initiation is delayed in normal $\mathrm{RT}$ trials to allow the brain to check the upcoming motor plan for errors.

Further insight into this separation was provided by Michaels et al. (2018). The population activity in premotor areas moves into a separate trajectory if a movement is planned, but execution has to be withheld. Movements that have to be executed as fast as possible follow a different trajectory in population space. However, both conditions meet during the execution of the action. This leads us to conclude that the upcoming motor plan is withheld in a holding pattern if the upcoming action has to be executed at a later stage (for more evidence for the existence of a withholding space in the population encoding in M1, see Jerjian et al., 2020).

To further investigate this question, we designed an experiment with different gocue conditions. In one condition, the instructed grasp has to be executed at an unpredictable point in time and the other at a predictable point in time. By assuring that the preceding movement planning phase is identical in both conditions, we aim to observe differences of the initiation process between both conditions. We hypothesize the observation of significant encoding in brain areas associated with motor working memory, movement initiation and movement execution.

Our second research question was whether hand orientation can be considered as part of the reach or the grasp process. Hand orientation by wrist rotation has been shown to be an integral part in reaching (Koshland et al., 2000). However, research in the neurological control of wrist rotation in humans is lacking. Monaco et al. (2011) only considered wrist adduction and abduction, but not wrist rotation. In NHPs, orientation-encoding neurons have been observed in AIP. F5, M1, and V6A (Murata et al., 2000, Baumann et al., 2009; Fattori et al., 2009, Fluet et al., 2010 Menz et al., 2015), with the authors generally attributing the orientation component to the grasp process. However, limb tracking studies in humans show that the hand orientation during a reach-to-grasp is dictated by a number of factors such as object location, object type, and intended grasp type (Touvet et al., 2014). Also, the wrist rotation component is independent from the reach components (Roby-Brami et al., 2003) and simultaneously adjusts along the preshaping of the hand early in the reach process (Sangole and Levin, 2008). 
This leaves the question open if hand orientation is part of the reach process, the grasp process, or completely independent from both. We aim to answer this question by examining an orientation condition, which requires the subject to rotate his or her hand, with the executed grasps remaining identical. The hypothesis is that wrist rotation and grasp are encoded in different brain areas, and wrist rotation is encoded in areas previously associated with reach processing in humans. This result would give strong support to the wrist rotation being part of the reach process and both being encoded in different streams as proposed in the two stream hypothesis (Jeannerod, 1981, Binkofski and Buxbaum, 2013). However, if we only find areas encoding the interaction effect between grasp and orientation, we would conclude that both are not separate processes.

Grasping in the MRI scanner is not often investigated. Most studies compare different reach-to-x movements incorporating an arm transport, such as Cavina-Pratesi et al. (2010b) (but also compare Cavina-Pratesi et al., 2007; Grol et al., 2007; Monaco et al., 2011) who compare reach-to-grasp, reach-to-touch and visual orientation towards the object. Since reaching and grasping show overlapping areas of connectivity in macaques (Greulich et al., 2020) and a strict separation between both processes is disputed (Kakei et al., 2001; Galletti et al., 2003, Fattori et al., 2004, Raos et al., 2004; Stark et al., 2007; Baumann et al., 2009; Fattori et al., 2009, 2010; Gamberini et al., 2011; Lehmann and Scherberger, 2013. Breveglieri et al., 2016), we question the validity of this approach. We therefore included a condition that only differs in the executed grasp. This condition allows us to test the validity of the previous results regarding grasping in humans utilizing modern MVPA decoding analysis.

To summarise, our research questions are as follows:

1. Which brain areas show significant encoding of the predictable vs. unpredictable go-cue?

2. Is the hand orientation condition encoded different from the grasp condition?

3. Where is information about the executed grasp encoded in the brain?

\subsubsection{Materials \& Methods}

\section{Task Design}

We used two different grasps for our experiment. A two-finger precision grasp and a full hand power grasp (Napier, 1956). Both grasps were executed either in a horizontal or a vertical orientation. The subject was instructed to rest its hand on 
the horizontal bar of the manipulandum. This results in the vertical condition to include a wrist rotation to align the hand with the vertical grasp target. While in the horizontal condition, the grasp can be executed directly.

For grasp initiation, we designed three different types of go-cues, consisting of the actual go-cue and different preceding stimuli. In all cases, the human subject was instructed to start the grasp only at the onset of the actual go-cue. For macaques, this condition is enforced by a rest button, which the animal has to keep pressed during the trial until the onset of the go-cue. In the unpredictable condition, the go-cue appears suddenly without the subject having temporal information. In the predictable condition, the go-cue is preceded by a pacing signal. The pacing signal is spaced $500 \mathrm{~ms}$ apart and presented for $100 \mathrm{~ms}$ (see figure 2.1 on the following page). To ensure that we do not decode the pacing signal in our analysis, we presented it in two different modalities, visual and auditory. The visual modality consists of the fixation dot turning blue and the auditory modality of a pure sine wave tone with $880 \mathrm{~Hz}$, each occuring for $100 \mathrm{~ms}$ and at an interval of $500 \mathrm{~ms}$

In total, we have two grasp conditions, two orientation conditions and three different go-cues. All conditions are within subject, leaving us with a $2 \times 2 \times 3$ factorial design.

We utilized a delayed response design (see figure 2.3 on page 70 ). A fixation dot was displayed for the duration of the whole experiment. After $1000 \mathrm{~ms}$ fixation dot, a conditional cue was underlaid under the fixation dot. The conditional cue consisted of a square in either a horizontal or vertical orientation, instructing the subject either to grasp on the horizontal or vertical bar of the manipulandum (see section 2.2 on page 40). The grasp was encoded by the color of the bar, red for a power grasp and green for a precision grasp(see figure 2.2 on page 69).

After the conditional cue, a variable delay ranging from $2000 \mathrm{~ms}$ up to $8000 \mathrm{~ms}$ was employed (termed intention period) to keep the following go-cue unpredictable for the subject. The go-cue consisted of the fixation dot turning from white to red. In the predictable condition, the go-cue was preceded by the pacing signal during the intention period. The go-cue was displayed for $500 \mathrm{~ms}$ and timed to conclude with the onset of a new echo planar imaging (EPI) volume. The trial concluded with a variable inter trial interval (ITI) from $2000 \mathrm{~ms}$ up to $6000 \mathrm{~ms}$.

The whole experiment was structured into four runs with 72 trails for each run. We also included 8 catch trials per run. Catch trials were identical to experimental trials with an unpredictable go-cue, but did not display a go-cue. All catch trials were not included in the analysis. The remaining 64 experimental trials were balanced over all conditions and arranged in a pseudo random order. Prior to scanning, the trial 


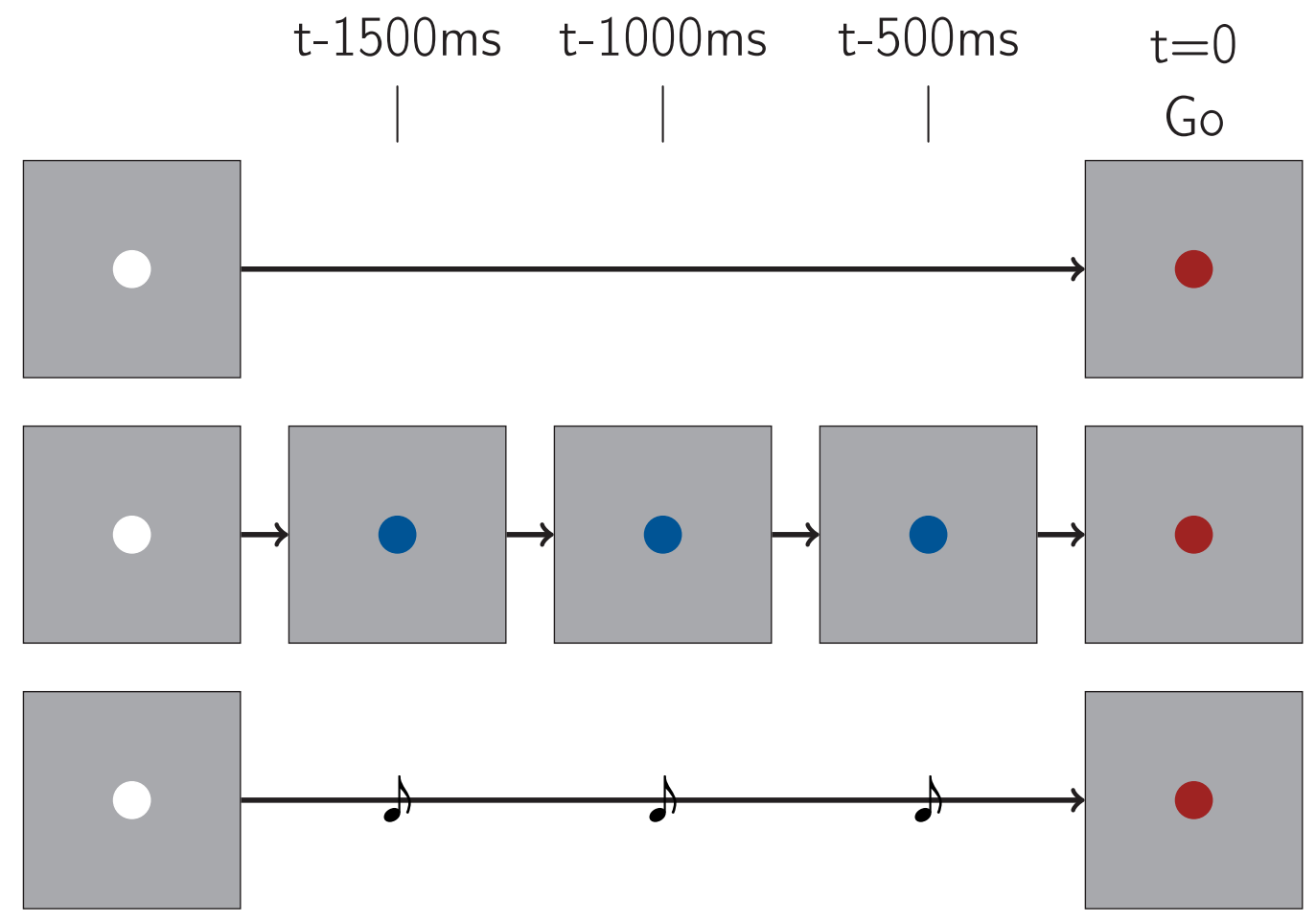

Figure 2.1.: Unpredictable (top), predictable visual (middle) and predictable auditory (bottom) go-cue design. In all three cases, the go-cue was the fixation dot turning from white to red. In the two predictable conditions, the go-cue was preceded by a pacing signal. In the predictable visual condition (middle) the pacing signal was the fixation dot turning blue three times for $100 \mathrm{~ms}$ with an interval of $500 \mathrm{~ms}$. The predictable auditory condition consisted of a pure sine wave tone of $880 \mathrm{~Hz}$ for $100 \mathrm{~ms}, 500 \mathrm{~ms}$ appart.

sequence was tested for sequence effects in line with the same analysis approach (Görgen et al., 2017). Each run took $12 \mathrm{~min}$ and $58 \mathrm{~s}$.

\section{Macaque Training}

Two male animals (both age 10 , weight $14.78 \& 11.25 \mathrm{~kg}$ ) are currently in training. Both have been habituated to the MRI chair and the experimental set-up. At the moment of writing this thesis, both are able to execute both grasps in the horizontal orientation when cued. We still need to train the grasps in the vertical orientation, fixation and habituate the animals to the scanner.

\section{Human participants}

We scanned 26 right handed participants (age 18-31, 20 female) for this study. Technical difficulties lead to an unusable session for three participants. One par- 


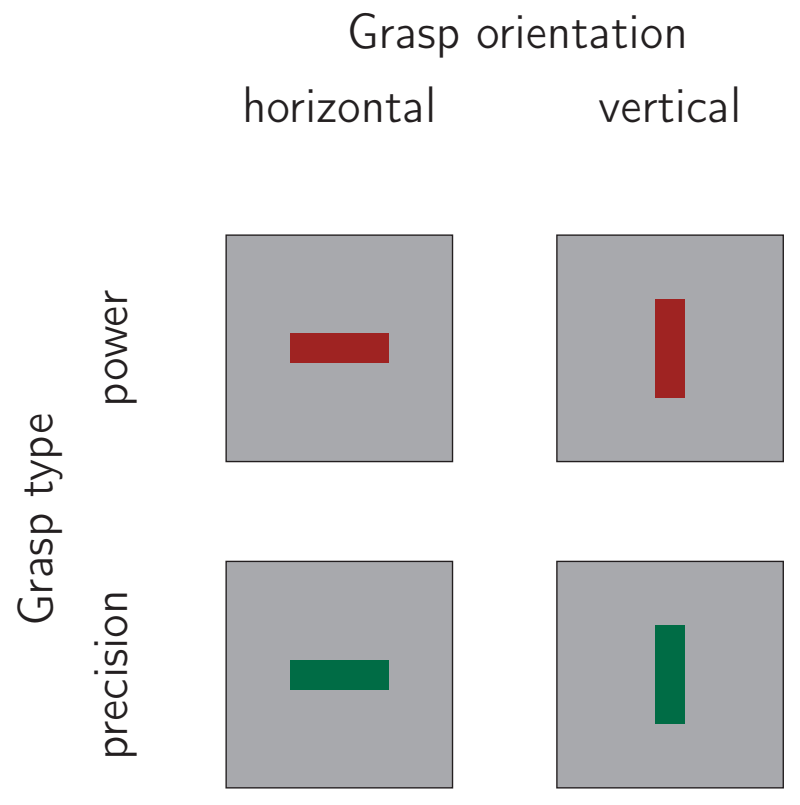

Figure 2.2.: Conditional cues for the grasp conditions. The orientation of the bar instructed the orientation of the grasp, horizontal bar-horizontal grasp and vertical bar-vertical grasp. The color instructed the grasp type, red for power grasp and green for precision grasp.

ticipant had excessive artifacts caused by a retainer. We excluded another three participants because of a high error rate (over $10 \%$ over all runs). Five participants showed excessive head movement (over $1 \mathrm{~mm}$ Euclidean distance between volumes). This leaves us with 14 subjects in total for the analysis (13 female). Handedness of the subjects was assessed with a German version of the Edinburgh Handedness inventory (Oldfield, 1971) and scored an average of 59 with a standard deviation (std) of 15 , indicating strong preference of the right hand.

\section{Ethical statement}

Animal care and training was conducted in accordance with German and European law, the Guidelines for the Care and Use of Mammals in Neuroscience and Behavioural Research (Council, 2003), and the NC3Rs Guidelines regarding Non-human primate accommodation, care and use (NC3Rs, 2017). The experiment was approved by the Animal Welfare Division of the Office for Consumer Protection and Food Safety of the State of Lower Saxony, Germany.

The human experiment was conducted in accordance with the Declaration of Helsinki and was approved by the ethics committee of the Georg Elias Müller Institute of Psychology of the University of Göttingen. All human participants provided written consent prior to experimentation and were financially compensated. 


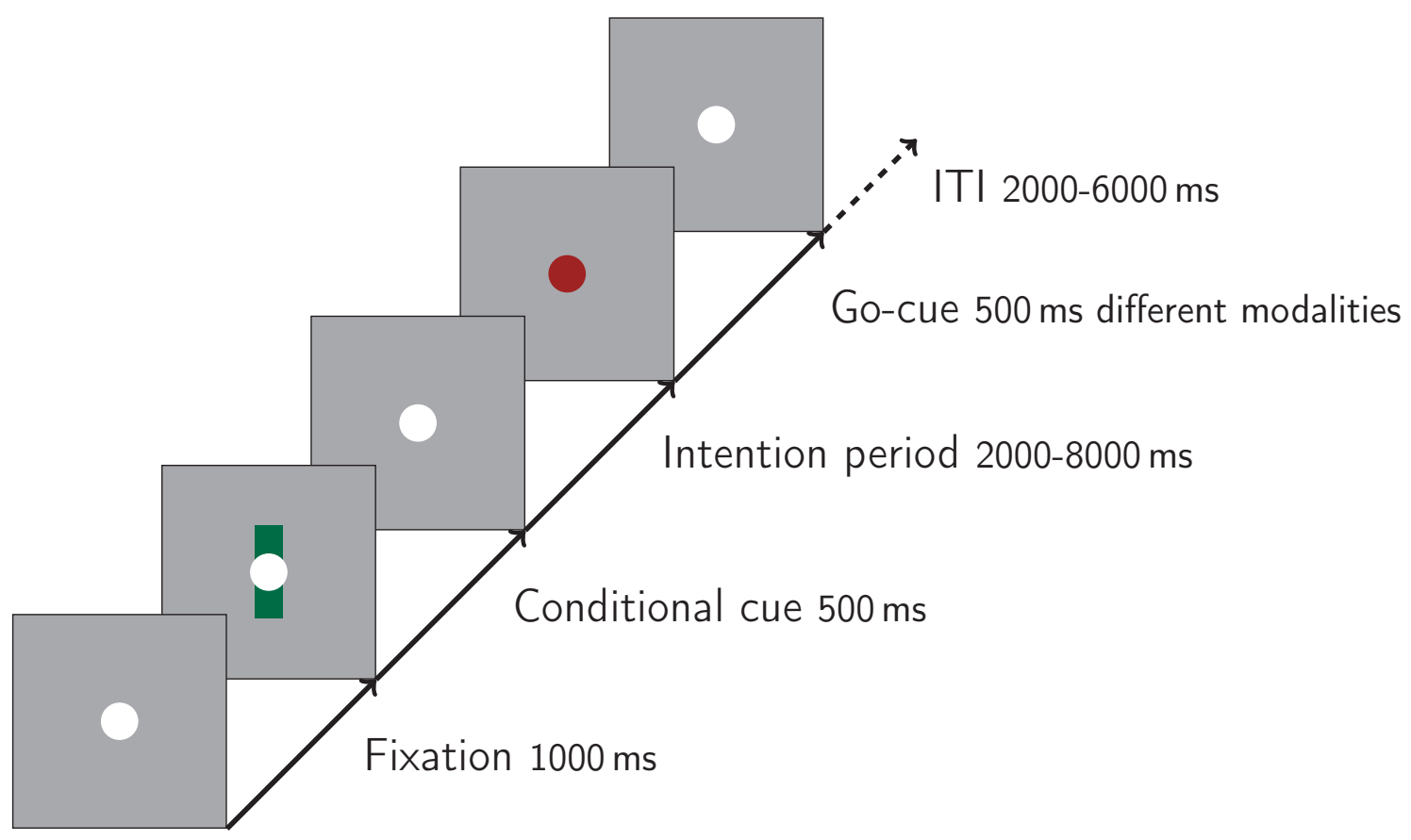

Figure 2.3.: Task paradigm. The trial started with a fixation dot for $1000 \mathrm{~ms}$, which is followed by a conditional cue underlayed for $500 \mathrm{~ms}$ instructing the grasp the subject had to execute in the ongoing trial. The following intention period had variable delays of $2000-4000-6000-8000 \mathrm{~ms}$ and included the pacing signal in the predictable go-cue conditions. The go-cue consisted of the fixation dot turning red for $500 \mathrm{~ms}$. The trial concluded with a inter trial interval (ITI) of 2000-4000-6000 ms.

\section{Color matching}

To further reduce visual confounds, all subjects underwent a color intensity matching procedure in the scanner. Two different colors were presented to to the subject in an alternating pattern with $10 \mathrm{~Hz}$ frequency. The intensity of one of those colors was adjusted continuously by the experimenter. The subject was instructed to indicate the point where only a color difference but no intensity difference was perceived. The colors were matched in the following pattern: green matched to red, white matched to red, and blue matched to white. This pattern was chosen to follow the presentation during the experiment as closely as possible. The stimulus of the color matching was the same size and shape as during the trial (bars for green to red, dots for the other two-color matches) to ensure that the perceived color intensity is the same as during the experiment. 


\section{Task training}

All subjects underwent task training in the scanner. We utilized a modified task design for this training. The intention period was shortened to $2 \mathrm{~s}$ and the ITI to $1 \mathrm{~s}$. Additionally, the subject was given feedback if the grasp was executed correctly in the ITI by displaying a green cross if the grasp was correct or a red cross if the grasp was incorrect. The training was closely monitored by the experimenter, to check for signs that the subject is moving before the go-cue (i.e. preparing the hand for the grasp) or not correctly returning to the rest position after the grip. All conditions were presented in random order and the whole training lasted for at least 72 trails.

\section{Subject questionnaire}

After scanning, subjects were given a questionnaire to rate the relative difficulty of the auditory vs. the visual modality and how unpredictable they perceived the gocue without the pacing signal. Both ratings were given a 5-point scoring. The first question ranging from 1-"auditory more difficult" to 5-"visual more difficult". The second question ranging from 1-"very suprising" ("sehr überraschend" in the original German) to 5-"not at all surprising" ("gar nicht überraschend").

\section{Data acquisition}

Human subjects were scanned with a 3 T Siemens Prisma scanner (Siemens, Erlangen) with a 20-channel coil.

We recorded a $T_{1}$ weighted magnetisation-prepared rapid gradient-echo (MPRAGE) for structural alignment of the subjects, with 176 slices, $1 \mathrm{~mm}$ slice distance, and a distance factor of 50\%. Each slice had a field of view (FoV) of $256 \mathrm{~mm}$ and a resolution of $1 \mathrm{~mm} \times 1 \mathrm{~mm}$.

For increased temporal resolution we utilised a $2 x$ multiband sequence for our $\mathrm{T}_{2}^{*}$ weighted $[\mathrm{EP} \mid$ volumes, resulting in a $\mathrm{TR}$ of $1 \mathrm{~s}$ with a $\mathrm{TE}=37 \mathrm{~ms}$. We used 32 slices with a slice thickness of $3 \mathrm{~mm}$ thickness and $10 \%$ distance factor to cover the whole brain. Phase encoding direction was right to left. The FoV was $192 \mathrm{~mm}$ and an in plane resolution of $3 \mathrm{~mm} \times 3 \mathrm{~mm}$. We recorded $778[\mathrm{EPI}$ volumes per run.

\section{Preprocessing}

For actual analysis, we used the EPI volumes that were motion corrected by the Siemens algorithm implemented in the MRI scanning software (Siemens, Erlangen). The uncorrected EPI volumes were spatially realigned with SPM 12 ( https: 
//www.fil.ion.ucl.ac.uk/spm/, Friston et al., 2007; runing on MATLAB Version: 9.5.0.1067069 (R2018b) Update 4 https://de.mathworks.com/products/ matlab.html) to extract the movement parameters. No smoothing or temporal realignment was done to preserve the spatial and temporal fine structure of the data.

Our setup was not designed to measure the onset of a movement (see previous project section 2.2 on page 40 ). Instead, we measured the movement time (MT), defined as the onset of the go-cue until the first button press. Subjects were specifically instructed to not start the movement before the go-cue and negative MTs and MTs over $4 \mathrm{~s}$ were counted as error trials. fMRl studies have to correct for differences in the trial-to-trial execution of the subject (Todd et al., 2013). We therefore calculated the variance of the MT $\mathrm{Mr}$ of each trial by subtracting the MT from the mean of the trials with the same condition regarding grasp and orientation. We did this only within condition combinations to not introduce a possible hidden confound between conditions.

$$
\mathrm{MT}_{\mathrm{var}}=\mathrm{MT}-\operatorname{mean}(\mathrm{MT})
$$

A stick function set at the start of the go-cue of each trial and with the height of the MT, value of the respective trial was convolved with the HRF and removed from the data as a regressor of non interest (implementation of the additive RT model from Woolgar et al., 2014).

In a decoding study, usually multiple instructional cues for the same conditions are used to reduce visual confounds (Reverberi et al., 2012). We decided against this, because training the monkey for multiple cues for the same conditions would multiply the training time. Since we intended to keep the human and macaque design as similar as possible, we also did not include this in the human experiment. We kept all visual stimuli small $\left(1-2^{\circ}\right.$ optical size) to minimize possible visual confounds. Additionally, the movement parameters (see above) were removed as regressors without convolving with the HRF.

\section{FIR model}

The trial was modelled with a general linear model with a finite impulse response (FIR) function as basis function in SPM 12. We included the movement parameters and other confounds as regressors of non interest (see previous section). We modelled each condition with 10 regressors of $1 \mathrm{~s}$ length each. The onset of the first regressor was set to the first volume recorded after the go-cue. For further analysis, we ignored the first two regressors. Those are within the movement onset of the subject and 
probably heavily contaminated by movement artifacts. We included all subsequent regressors in the following analysis.

\section{CVMANOVA}

Data was analyzed with searchlight based multi-voxel pattern analysis (Kriegeskorte et al., 2006) by cross-validated MANOVA (cvMANOVA, Allefeld and Haynes, 2014). cvMANOVA is especially suited for multi factorial experimental paradigms. Traditional decoding approaches are based on a classifier categorizing the signal as belonging to one of multiple conditions, which makes decoding interactions difficult. CVMANOVA avoids that pitfall entirely by using the multivariate variant of ANOVA (MANOVA, Timm, 2002)) to analyze the structure of the data directly. The measurement of this data structure difference is based on the Mahalanobis distance and has been termed pattern distinctness (Allefeld and Haynes, 2014).

For the main analysis, we pulled the predictable go-cue over both conditions (visual \& auditory) and termed this as a reduced effect predictability. We picked the term reduced effect, since it is reducing the conditions from 3 to 2 . To check for confounds between both conditions, the main effect visual predictor vs. auditory predictor was included as a separate contrast. We defined our contrast vectors for the following effects:

1. main effect grasp orientation

2. main effect grasp type

3. reduced main effect predictability

4. interaction grasp type $\times$ orientation

5. reduced interaction grasp orientation $\times$ predictability

6. reduced interaction grasp type $\times$ predictability

7. reduced interaction grasp type $\times$ orientation $\times$ predictability

8. main effect visual vs. auditory

The contrast vectors were expanded to cover multiple regressors of our FIR model (see previous section) in the same way as done by Christophel et al. (2018). Calculation of the pattern distinctness maps were performed in subject space with a searchlight radius of 3 voxels. We used the cvMANOVA MATLAB toolbox provided by Dr. Allefeld (https://github.com/allefeld/cvmanova) for all cvMANOVA calculations. 


\section{Crosssubject coregistration}

The $T_{1}$ volume of each subject was corregistrated with the $E P \mid$ volumes by normalized mutual information (as implemented in SPM 12). Normalisation parameters for each subject to the Montreal Neurological Institute (MNI) space was calculated by coregistration of the $T_{1}$ MP-RAGE to the International Consortium of Brain Mapping (ICBM) template from SPM 12 (Grabner et al., 2006). Those parameters were used to reslice the pattern distinctness maps of each subject into MNI space with a voxel size of $2 \times 2 \times 2 \mathrm{~mm}$ and subsequently smoothed with a Gaussian smoothing kernel (full width at half maximum (FWHM) $=8 \times 8 \times 8 \mathrm{~mm}$ ) as it is recommend for the subsequent population statistic (Allefeld et al., 2016). All reported coordinate values in this study are in accordance with the MNI standard.

\section{Population statistics}

Since information measurements do not follow a normal distribution and as such are not suitable for the standard fMRI analysis methods such as t-test (Allefeld et al., 2016), we utilized prevalence inference with a nonparametric permutation test in this study (Nichols and Holmes, 2002, Allefeld et al., 2016). Prevalence inference tests if a certain proportion larger than $\gamma$ of the general population has information present in the brain, based on the collected subsample in the experiment. The described statistics from Allefeld et al. (2016) were implemented for the population analysis. The a priori decision values required were population prevalence, set to $\gamma=0.5$, and the rejection threshold of the null-hypothesis, set to $\alpha=0.05$ corrected for multiple comparison. A non parametric permutation testing (Nichols and Holmes, 2002) was used as second level test statistic.

Our null-distribution for the population statistics (second level permutations) was constructed from all possible permutations of the signs of the contrast vector for the individual runs (first level permutations), resulting in 8 possible first level permutations for each subject. The pattern distinctness maps for each first level permutation were calculated with CvMANOVA. Since the number of all possible second level permutations was too large $\left(8^{14}>4 \times 10^{14}\right)$ to be considered in its entirety, we used a Monte Carlo estimation. We randomly drew $10^{5}$ permutations consisting of on permutation map per subject for our null-distribution (Stelzer et al., 2013). These permutation maps were used to calculate the voxel wise p-value corrected for multiple comparison for our test for information prevalence (Nichols and Holmes, 2002 Allefeld et al., 2016). This process was done for each contrast separately. 


\section{Identification of anatomical structures and rendering}

We used the automated anatomical labelling atlas third version (AAL) to identify the location of our significant voxels (Tzourio-Mazoyer et al., 2002). For locations in the white matter, we indicated the closest gyrus or anatomical structure in brackets. We used MRIcroGL ver. 1.2.20200331 Cocoa for all renderings and slice figures (https://www.mccauslandcenter.sc.edu/mricrogl/home). BAs were identified by utilising the Brodmann labeled map provided in the RESTplus toolbox (V 1.1; Jia et al., 2019) for SPM 12.

\subsubsection{Results}

\section{Subject questionnaire \& behavioral results}

Subjects consistently rated the visual modality as more difficult than the auditory modality (mean 4.00 , std 0.53 on our 5 point scale). The unpredictable go-cue was rated as averagely surprising (mean 3.14, std 0.99 on our 5 point scale).

Subjects showed a low error rate of $1.8 \%$ in average with a std of $1.2 \%$ over all 4 runs.

The mean MT over all conditions was $1.33 \mathrm{~s}$ with a std of $0.48 \mathrm{~s}$. We analyzed the MTF across subjects between all grasp types, orientation, and visual predictable vs auditory predictable in a three-way ANOVA to check whether the perceived difference in difficulty was reflected in the MT (see table 2.1 on the next page). None of the effects of this ANOVA were significant. However, the visual predictable vs auditory predictable main effect showed the lowest $p$-value with $p=0.12(F(1,104)=2.5)$.

We performed another three-way ANOVA (see table 2.2 on the following page) where we collapsed over the predictable conditions as in the CVMANOVA. We tested for the main effect's predictability, grasp, orientation, and all interaction effects over all subjects. The main effect predictability was highly significant $(F(1,104)=27.42$, $\left.p=8.6 \times 10^{-7}\right)$. All other effects did not cross the significance threshold, but the orientation main effect showed the second lowest p-value of $0.10(F(1,104)=2.69)$.

\section{Main effect grasp orientation}

For the grasp orientation contrast, we see one cluster of significant voxels on the left postcentral gyrus (see table 2.3 on page 77 and figure 2.5 on page 78). It is located within $\mathrm{BA} 2$ and the rostral subdivision of $\mathrm{BA} 7$. 
Table 2.1.: ANOVA for visual vs auditory $\mathrm{MT}$ s over all subjects. Only MTs for the visual predictable and auditory predictable go-cue were considered to test for a significant effect between those two conditions.

\begin{tabular}{lrrrrrr}
\hline Effect & Sum Sq. & d.f. & Mean Sq. & & F & p-value \\
\hline Main Go Cue (Visual $\times$ Auditory) & 0.5092 & 1 & 0.50924 & 2.5 & 0.1169 \\
Main Grasp & 0.1078 & 1 & 0.10784 & 0.53 & 0.4685 \\
Main Orientation & 0.2872 & 1 & 0.28722 & 1.41 & 0.2378 \\
Interaction Go Cue $\times$ Grasp & 0.0005 & 1 & 0.00055 & 0 & 0.9588 \\
Interaction Go Cue $\times$ Orientation & 0.0002 & 1 & 0.00023 & 0 & 0.9732 \\
Interaction Grasp $\times$ Orientation & 0.2339 & 1 & 0.23393 & 1.15 & 0.2864 \\
Interaction & 0.0107 & 1 & 0.01074 & 0.05 & 0.8188 \\
Go Cue $\times$ Grasp $\times$ Orientation & & & & & \\
\hline Error & 21.1884 & 104 & & & \\
Total & 22.3382 & 111 & & & \\
\hline
\end{tabular}

Table 2.2: ANOVA for predictable vs unpredictable go-cue type over all subjects. Both predictable conditions were collapsed for this analysis to stay in line with the main $\mathrm{fMRI}$ analysis. We collapsed by averaging for each subject over both predictable conditions.

\begin{tabular}{lrrrrrr}
\hline Effect & Sum Sq. & d.f. & Mean Sq. & F & p-value \\
\hline Main Predictability & 5.0328 & 1 & 5.03282 & 27.42 & $<0.0001$ \\
Main Grasp & 0.1328 & 1 & 0.13277 & 0.72 & 0.397 \\
Main Orientation & 0.493 & 1 & 0.49298 & 2.69 & 0.1043 \\
Interaction Predictability $\times$ Grasp & 0.0013 & 1 & 0.00127 & 0.01 & 0.9338 \\
Interaction & 0.0276 & 1 & 0.02757 & 1.19 & 0.2775 \\
Predictability $\times$ Orientation & & & & & \\
Interaction Grasp $\times$ Orientation & 0.2188 & 1 & 0.21878 & 1.19 & 0.2775 \\
Interaction & 0.0003 & 1 & 0.00026 & 0 & 0.9698 \\
Predictability $\times$ Grasp $\times$ Orientation & & & & & \\
\hline Error & 19.0907 & 104 & & & \\
Total & 24.9972 & 111 & & & \\
\hline
\end{tabular}




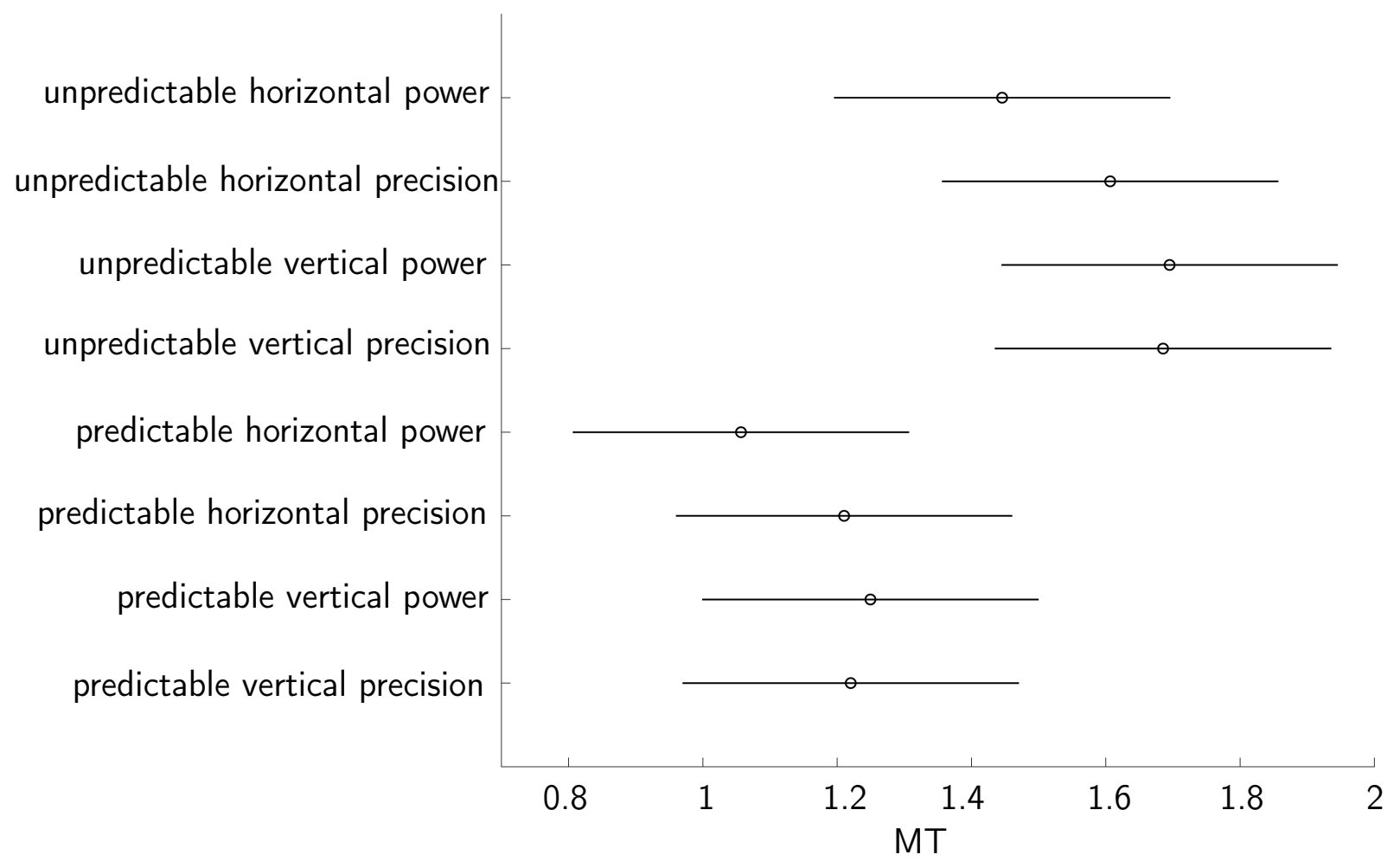

Figure 2.4.: Plot of the $\mathrm{MT}$ distributions of the ANOVA for predictable vs unpredictable go-cue type over all subjects (see table 2.2 on the preceding page). Both predictable conditions were collapsed as described in the text and table 2.2 on the facing page. The circles represent the mean and the bars the $95 \%$ confidence interval of each distribution.

Table 2.3.: Main effect grasp orientation. Location of the lowest p-value (voxel wise corrected for multiple comparison by permutation analysis with 100000 permutations) and size of surrounding cluster in voxels. Anatomical labelling as reported by the AAL atlas. The labels are sorted by proportion within the cluster, with the label of the peak location in italics.

\begin{tabular}{|c|c|c|c|c|c|}
\hline \multirow[t]{2}{*}{ Anatomical Area } & \multirow[t]{2}{*}{ Size } & \multicolumn{3}{|c|}{ Peak Coordinates } & \multirow{2}{*}{$\mathrm{P}_{\text {peak }}$} \\
\hline & & $X$ & $Y$ & Z & \\
\hline $\begin{array}{l}\text { postcentral gyrus, superior parietal lobule, } \mathrm{BA} \\
2,7)\end{array}$ & 16 & -30 & -44 & 66 & 0.002 \\
\hline
\end{tabular}


p-value
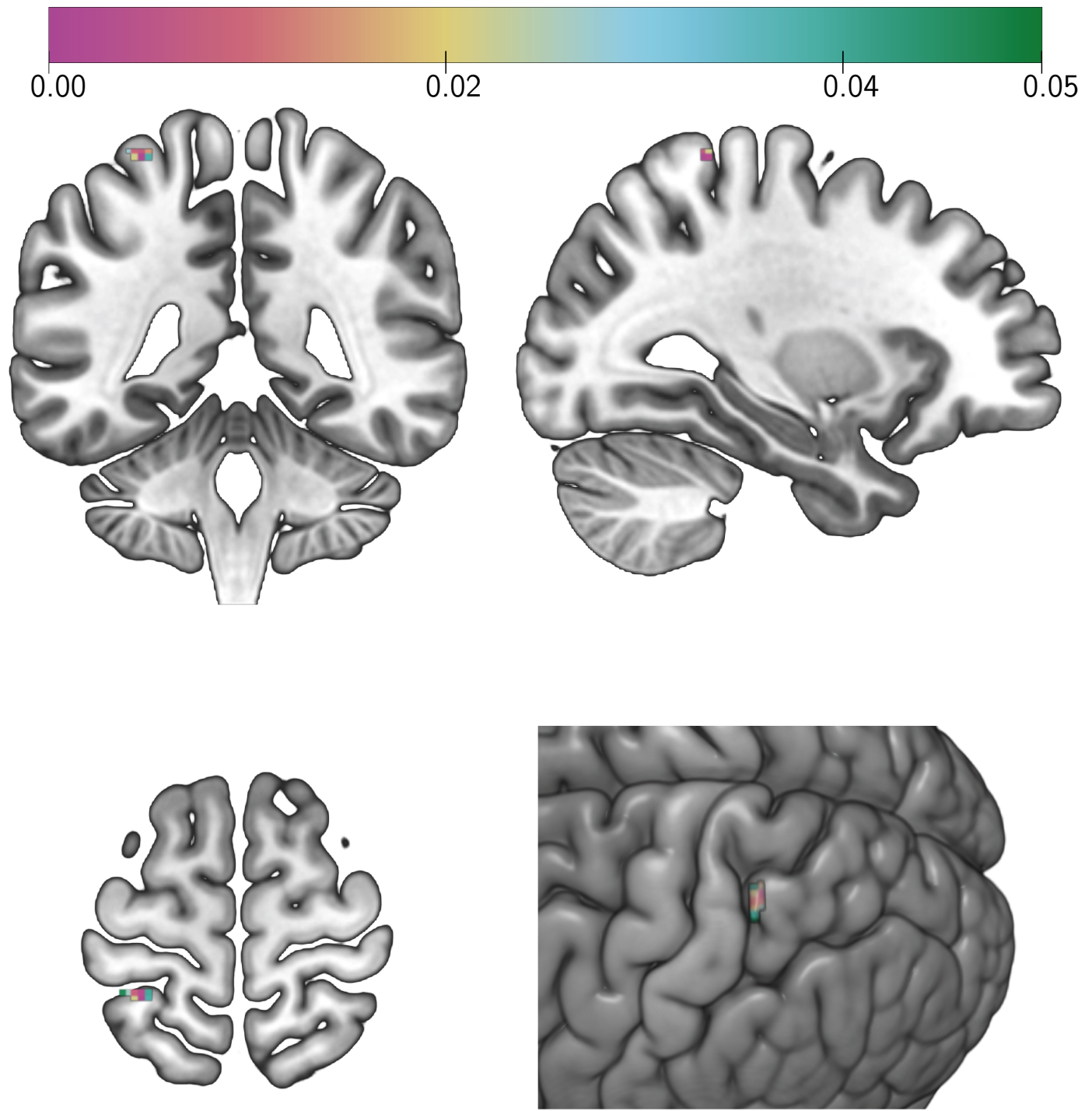

Figure 2.5.: Main effect grasp orientation. Location of the significant voxels (thresholded with $\mathrm{p}=0.05$, voxel wise corrected for multiple comparison by permutation analysis with 100000 permutations) within the MNI 152 standard brain template. Coloration indicates the p-value of the voxel according to the displayed color bar. Axial and coronal slices use neurological convention (left on the left). 


\section{Main effect grasp type}

In the main effect grasp type, three clusters of significant voxel are visible (see table 2.4 and figure 2.6 on the following page). All clusters are on the left hemisphere contralateral to the used hand. They are located along the central sulcus mainly within the postcentral gyrus, covering $\mathrm{BA} s 3,4$, and 6 . The clusters are at a comparable height along the postcentral gyrus, where the intraparietal sulcus meets the postcentral gyrus.

Table 2.4.: Main effect grasp type. Location of the lowest p-value (voxel wise corrected for multiple comparison by permutation analysis with 100000 permutations) and size of surrounding cluster in voxels. The clusters are sorted by ascending order for the peak p-value. The labels are sorted by proportion within the cluster, with the label of the peak location in italics.

\begin{tabular}{|c|c|c|c|c|c|}
\hline \multirow[t]{2}{*}{ Anatomical Area } & \multirow[t]{2}{*}{ Size } & \multicolumn{3}{|c|}{ Peak Coordinates } & \multirow[t]{2}{*}{$P_{\text {peak }}$} \\
\hline & & $x$ & Y & Z & \\
\hline $\begin{array}{l}\text { precentral gyrus, postcentral gyrus, } \mathrm{BA} 4,3 \text {, } \\
6)\end{array}$ & 179 & -36 & -26 & 58 & $<0.001$ \\
\hline postcentral gyrus, $(\mathrm{BA} 4,6)$ & 12 & -52 & -14 & 48 & 0.008 \\
\hline precentral gyrus, postcentral gyrus, $(\mathrm{BA} 4,6)$ & 4 & -44 & -14 & 60 & 0.045 \\
\hline
\end{tabular}

\section{Reduced main effect predictability}

The reduced contrast produced 25 clusters of significant voxels and 7 individual significant voxels (see table 2.5 on page 82 and figure 2.7 on page 84). The clusters above four significant voxels are reported in ascending order of the peak p-value.

The first cluster showing the lowest peak p-value was located within the right superior temporal gyrus and additionally showed significant voxels inside the supramarginal gyrus and Rolandic operculum. It stretched over a range of BAs, namely 48, 42, 22, 2, and 40. The second and largest cluster lies within the left precentral, postcentral and middle frontal gyrus. Both the precentral and the postcentral gyrus showed an individual peak of the p-value within this cluster. The covered BAs were $6,44,4,9$, and 3 in descending order of covered area. The third and second largest cluster was found on the right hemisphere over the insula, Rolandic operculum and Heschl's gyrus. The peak was within the Rolandic operculum and the whole cluster was also exclusively within $\mathrm{BA}$ 48. The fourth by peak significance and third largest cluster was over the left SMA and middle cingulate gyrus, within BA 6 \& 4 and the peak within the SMA. Within the left temporal lobe over the superior temporal, 
p-value
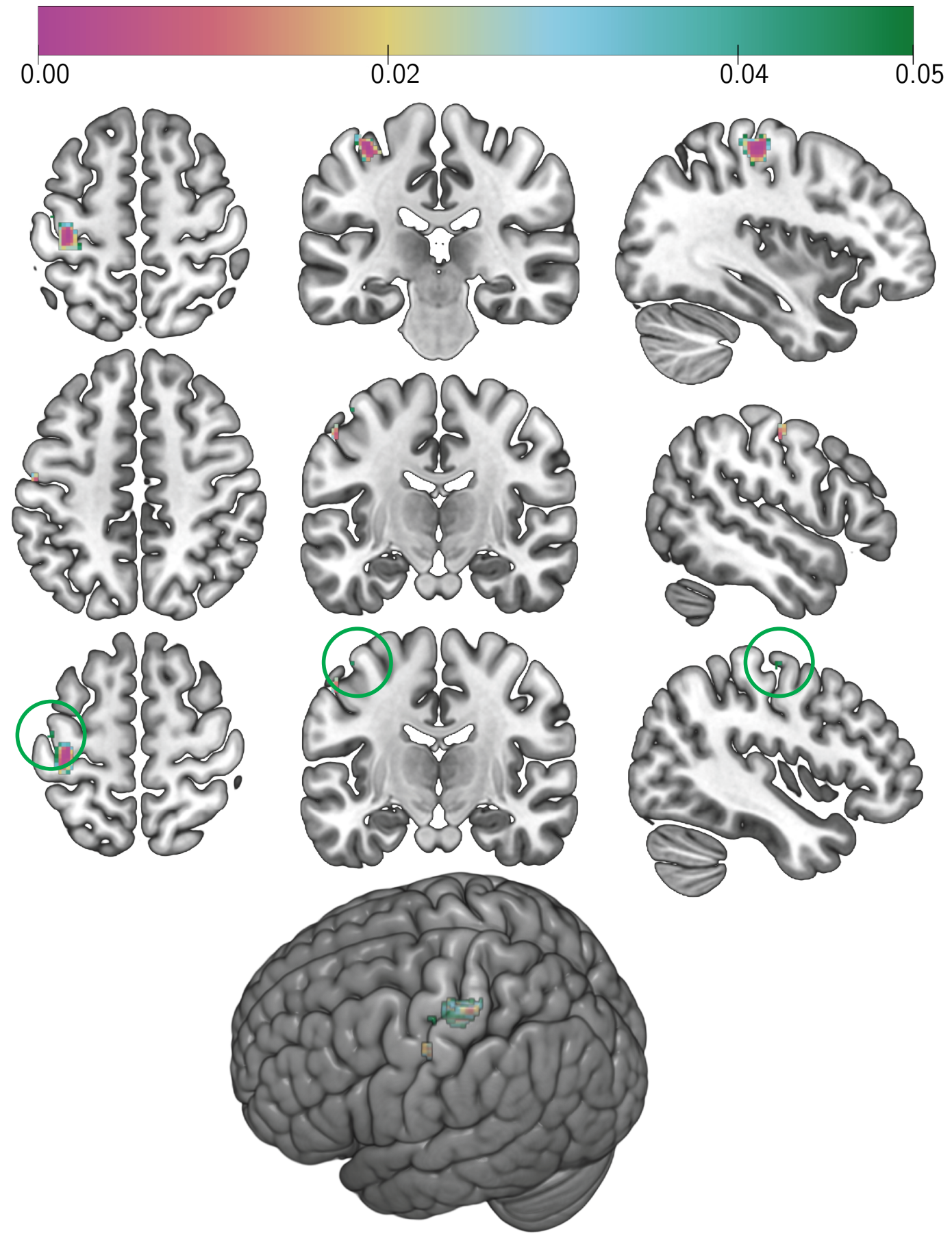

Figure 2.6.: Main effect grasp Type. Location of the significant voxels (thresholded with $p=0.05$, voxel wise corrected for multiple comparison by permutation analysis with 100000 permutations) within the MNI152 standard brain template. Each row of sections is cut through the coordinates of the peak p-value of one cluster (see table 2.4 on the preceding page, clusters in the same order, top to bottom, as in the table). Coloration indicates the p-value of the voxel according to the displayed color bar. Axial and coronal slices use neurological convention (left on the left). Green circles highlight positions of small clusters. 
Heschl's gyrus, and Rolandic operculum was the fifth cluster located, with the peak p-value in Heschl's gyrus and covering BA 48. The sixth cluster was exactly on the center line at the SMA reaching down to the middle cingulate gyrus and up to the superior frontal gyrus. $\mathrm{BA}_{5} 6,32,8$, and 24 were found within this cluster. On the right hemisphere at the middle frontal, superior frontal, and precentral gyrus was the fourth cluster, located wholly within $\mathrm{BA} 6$. The peak p-value of this cluster was within the middle frontal gyrus. The eighth cluster was in the left hemisphere over the superior frontal gyrus and the SMA and was within $\mathrm{BA}$ 6. Stretching over the left paracentral lobule, precentral, postcentral and supperior frontal gyrus is the ninth cluster by p-value and sixth by size. It covers $\mathrm{BA} \$ 55$ and 4 , with the peak located within the postcentral gyrus. In the right hemisphere at the contralateral location of the fifth was the tenth cluster. With the peak in the superior temporal gyrus and touching the Rolandic operculum and insula. Additionally to $\mathrm{BA} 48$, the cluster had significant voxels within $\mathrm{BA} 41$ and 21 . Another cluster was located in the right occipital lobe within the middle and superior occipital gyrus and was mainly within BA 19 with one voxel within BA 18. One cluster was located within the right SMA and $B A$ 6. In the right temporal superior gyrus, touching the Rolandic operculum was a cluster covering $\mathrm{BA}_{3} 22$ and 48 . On the posterior right hemisphere was the last cluster with more than 20 significant voxels. Located at the superior parietal gyrus, extending to the superior occipital gyrus and precuneus. This cluster was completely within $\mathrm{BA}$ 7. The left insula had another cluster within $\mathrm{BA}$ 48. The most frontal cluster was found in the right superior frontal gyrus in $B A \$ 10$ and 11 . An additional cluster was on the right precentral gyrus on $\mathrm{BA}_{5} 6,9$, and 44 . A further small cluster on the right postcentral gyrus in $\overline{B A} \$ 4$ and 3 . The last cluster above 4 significant voxels is within the right cerebellum (vermis and cerebal hemisphere) and the lingual gyrus. This cluster had significant voxels within BA 18,30 , and 27.

The resulting clusters of four or two voxel size as well as the individual voxels are reported here in no particular order. There are three clusters with a voxel size of four voxels. One is within the left postcentral gyrus (BAs $3 \& 4)$, another within the right precentral gyrus $(B A s 4$ \& 3$)$, and within the right superior parietal gyrus $(B A 7)$. A range of two voxel clusters were on the right hemisphere in the right middle frontal gyrus (BA $46 \& 9$ ), superior parietal gyrus (BA 7) and the white matter under the precentral sulcus. In the left hemisphere a single cluster of two voxel size was loacted in the white matter under the inferior frontal operculum.

We found individual significant voxels in a range of brain areas. Those were within the left middle cingulate gyrus (BA 24), the right middle occipital gyrus (BA 19), the right cuneus $(\mathrm{BA} 18)$, the right superior and inferior parietal gyrus (both $B A 2$ ), 
the left postcentral (BA 3$)$ and middle frontal gyrus $(\mathrm{BA} 6)$.

Table 2.5.: Reduced main effect predictability. Location of the lowest p-value (voxel wise corrected for multiple comparison by permutation analysis with 100000 permutations) and size of surrounding cluster in voxels. The clusters are sorted by ascending order for the peak p-value. The labels are sorted by proportion within the cluster, with the label of the peak location in italics.

\begin{tabular}{|c|c|c|c|c|c|}
\hline \multirow[t]{2}{*}{ Anatomical Area } & \multirow[t]{2}{*}{ Size } & \multicolumn{3}{|c|}{ Peak Coordinates } & \multirow[t]{2}{*}{$p_{\text {peak }}$} \\
\hline & & $x$ & Y & Z & \\
\hline $\begin{array}{l}\text { supramarginal gyrus, superior temporal gyrus, } \\
\text { Rolandic operculum, (BA } 48,42,22,2,40)\end{array}$ & 223 & 64 & -28 & 18 & $<0.001$ \\
\hline precentral gyrus, & 798 & -48 & 6 & 40 & $<0.001$ \\
\hline $\begin{array}{l}\text { postcentral gyrus, middle frontal gyrus, } \mathrm{BA} 6 \text {, } \\
44,4,9,3)\end{array}$ & & -44 & -10 & 52 & $<0.001$ \\
\hline $\begin{array}{l}\text { insula, Rolandic operculum, Heschl's gyrus, su- } \\
\text { perior temporal gyrus, putamen, (BA 48) }\end{array}$ & 264 & 48 & -10 & 12 & $<0.001$ \\
\hline SMA middle cingulate gyrus, $(\mathrm{BA} 6,4)$ & 136 & -8 & -12 & 56 & $<0.001$ \\
\hline $\begin{array}{l}\text { superior temporal gyrus, Heschl's gyrus, } \\
\text { Rolandic Operculum, insula, (BA 48) }\end{array}$ & 74 & -46 & -18 & 6 & $<0.001$ \\
\hline $\begin{array}{l}\text { SMA middle cingulate gyrus, superior frontal } \\
\text { gyrus (medial part), (BA } 6,32,8,24)\end{array}$ & 135 & 0 & 16 & 54 & $<0.001$ \\
\hline $\begin{array}{l}\text { middle frontal gyrus, precentral gyrus, superior } \\
\text { frontal gyrus, (BA 6) }\end{array}$ & 93 & 42 & -4 & 60 & $<0.001$ \\
\hline superior frontal gyrus, SMA (BA 6) & 121 & -16 & 8 & 68 & $<0.001$ \\
\hline $\begin{array}{l}\text { precentral gyrus, paracentral lobule, postcen- } \\
\text { tral gyrus, supperior frontal gyrus, }(\mathrm{BA} 55,4)\end{array}$ & 131 & -18 & -26 & 80 & $<0.001$ \\
\hline $\begin{array}{l}\text { superior temporal gyrus, Rolandic operculum, } \\
\text { insula, (BA } 41,48,21)\end{array}$ & 47 & 44 & -34 & 14 & 0.001 \\
\hline middle occipital gyrus, superior occipital gyrus, & 18 & 34 & -80 & 16 & 0.002 \\
\hline SMA $(\mathrm{BA} 6)$ & 16 & 6 & -12 & 76 & 0.005 \\
\hline $\begin{array}{l}\text { superior temporal gyrus, Rolandic operculum, } \\
\text { BA 22, 48) }\end{array}$ & 9 & 62 & -16 & 10 & 0.007 \\
\hline $\begin{array}{l}\text { superior parietal gyrus, superior occipital gyrus, } \\
\text { precuneus, }(\overline{B A} 7)\end{array}$ & 26 & 20 & -64 & 50 & 0.008 \\
\hline
\end{tabular}

Continues on next page 
Table 2.5.: Reduced main effect predictability continued.

\begin{tabular}{|c|c|c|c|c|c|}
\hline \multirow[t]{2}{*}{ Anatomical Area } & \multirow[t]{2}{*}{ Size } & \multicolumn{3}{|c|}{ Peak Coordinates } & \multirow[t]{2}{*}{$\mathrm{p}_{\text {peak }}$} \\
\hline & & $x$ & Y & Z & \\
\hline insula, superior temporal pole, $(\mathrm{BA} 48)$ & 13 & -44 & 10 & -6 & 0.009 \\
\hline superior frontal gyrus, $(\mathrm{BA} 10,11)$ & 6 & 20 & 58 & 2 & 0.013 \\
\hline $\begin{array}{l}\text { precentral gyrus, middle frontal gyrus, } \mathrm{BA} 6 \text {, } \\
9,44)\end{array}$ & 9 & 54 & 12 & 42 & 0.014 \\
\hline postcentral gyrus, (BA 3, 4) & 4 & -44 & -26 & 58 & 0.014 \\
\hline middle frontal gyrus, $(\mathrm{BA} 46,9)$ & 2 & 40 & 38 & 38 & 0.016 \\
\hline middle cingulate gyrus, (BA 24) & 1 & -10 & 2 & 40 & 0.017 \\
\hline $\begin{array}{l}\text { vermis }(I V, V) \text {, cerebelum }(I V, V) \text {, lingual gyrus, } \\
(\operatorname{BA} 18,30,27)\end{array}$ & 12 & 8 & -50 & -2 & 0.019 \\
\hline postcentral gyrus, precentral gyrus, $(\mathrm{BA} 4,3)$ & 4 & 48 & -16 & 50 & 0.020 \\
\hline superior parietal gyrus, (BA 7) & 4 & 30 & -60 & 60 & 0.021 \\
\hline middle occpital gyrus, (BA 19) & 1 & 30 & -82 & 20 & 0.021 \\
\hline white matter (precentral gyrus) & 2 & 32 & -18 & 52 & 0.029 \\
\hline cuneus, BA 18) & 1 & 12 & -76 & 24 & 0.036 \\
\hline white matter (inferior frontal operculum) & 2 & -34 & 6 & 20 & 0.038 \\
\hline superior parietal gyrus, (BA 7) & 2 & 22 & -62 & 64 & 0.043 \\
\hline superior parietal gyrus, (BA 2) & 1 & 50 & -36 & 58 & 0.044 \\
\hline inferior parietal gyrus, (BA 2) & 1 & 52 & -34 & 56 & 0.045 \\
\hline postcentral gyrus, (BA 3) & 1 & -42 & -24 & 54 & 0.046 \\
\hline middle frontal gyrus, (BA 6) & 1 & -36 & 10 & 56 & 0.049 \\
\hline
\end{tabular}

\section{Interaction grasp type $\times$ orientation}

For the interaction effect of grasp type and orientation we found one small cluster of significant voxels (see table 2.6 on page 85 and figure 2.8 on page 86 ). This cluster was fully within the left postcentral gyrus $(\mathrm{BA} 3,2, \& 4)$.

\section{Reduced interaction grasp orientation $\times$ predictability}

In the reduced interaction effect grasp $\times$ predictability, we found four clusters of significant voxels (see table 2.7 on page 85 and figure 2.9 on page 87 ). The second largest cluster was between both hemispheres in the central fissure over the SMA of both hemispheres. It also showed the lowest peak p-value and was over $\operatorname{BA} \beta 6$ \& 4 . 


\section{p-value}
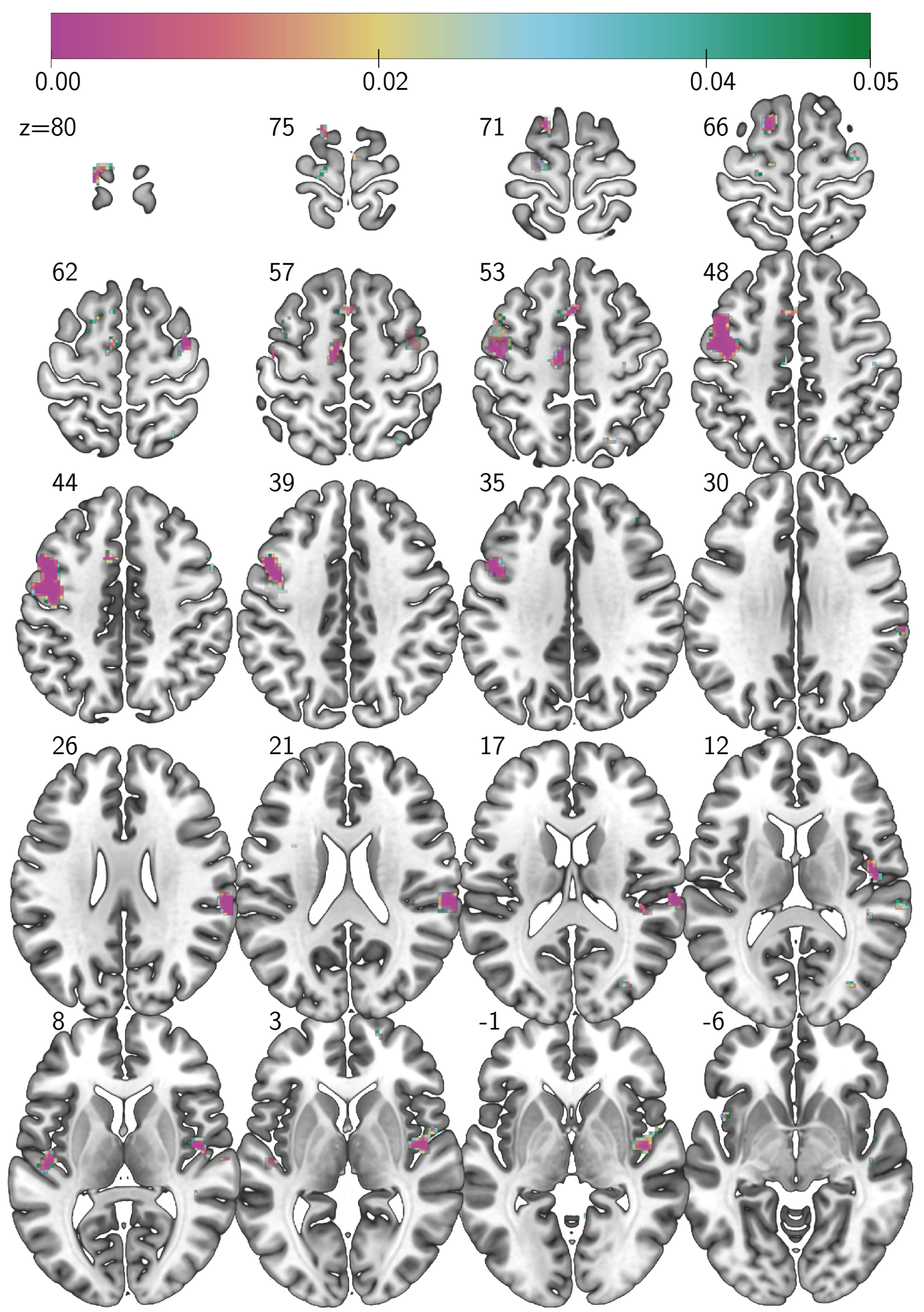

Figure 2.7.: Reduced main effect predictability. See facing page. 
Figure 2.7.: Continued from facing page. Reduced main effect predictability. Location of the significant voxels (thresholded with $p=0.05$, voxel wise corrected for multiple comparison by permutation analysis with 100000 permutations) within the MNI152 standard brain template. Coloration indicates the $\mathrm{p}$-value of the voxel according to the displayed color bar. Slices use neurological convention (left on the left). Z values of the slices are stated above left of the respective slice.

Table 2.6.: Interaction effect grasp type $\times$ orientation Location of the lowest p-value (voxel wise corrected for multiple comparison by permutation analysis with 100000 permutations) and size of surrounding cluster in voxels.

\begin{tabular}{|c|c|c|c|c|c|}
\hline \multirow{2}{*}{ Anatomical Area } & \multirow[t]{2}{*}{ Size } & \multicolumn{3}{|c|}{ Peak Coordinates } & \multirow{2}{*}{$\mathrm{p}_{\text {peak }}$} \\
\hline & & $x$ & Y & Z & \\
\hline postcentral gyrus, $(\mathrm{BA} 3,2,4)$ & 10 & -32 & -38 & 64 & 0.006 \\
\hline
\end{tabular}

The largest cluster and showing the second lowest peak p-value was directly midline on the corpus callosum and superior part of the anterior cingulate cortex (ACC). In the left occipital lobe was the third largest cluster of significant voxels. It was mainly over the calcarine fissure, but had the lowest p-value within the superior occipital gyrus. Additional involved structures were the cuneus and middle occipital gyrus, with $\mathrm{BA} \leqslant 17$ and 18 . The fourth cluster was the smallest and has the highest peak p-value from all four clusters. It lay within the cuneus and precuneus on the right hemisphere $(\mathrm{BA} \$ 18$ and 19).

Table 2.7.: Interaction effect grasp orientation $\times$ predictability Location of the lowest $p$-value (voxel wise corrected for multiple comparison by permutation analysis with 100000 permutations) and size of surrounding cluster in voxels. The labels are sorted by proportion within the cluster, with the label of the peak location in italics.

\begin{tabular}{|c|c|c|c|c|c|}
\hline \multirow[t]{2}{*}{ Anatomical Area } & \multirow[t]{2}{*}{ Size } & \multicolumn{3}{|c|}{ Peak Coordinates } & \multirow[t]{2}{*}{$p_{\text {peak }}$} \\
\hline & & $x$ & Y & Z & \\
\hline SMA, paracentral lobule, $(\mathrm{BA} 6,4)$ & 49 & 0 & -12 & 64 & 0.003 \\
\hline white matter (corpus callosum), superior ACC & 69 & 0 & 8 & 22 & 0.003 \\
\hline $\begin{array}{l}\text { calcarine fissure, superior occipital gyrus, } \\
\text { cuneus, middle occipital gyrus, (BA } 17,18)\end{array}$ & 41 & -12 & -90 & 10 & 0.004 \\
\hline cuneus, precuneus, $\mathrm{BA} 18,19)$ & 4 & 22 & -66 & 26 & 0.023 \\
\hline
\end{tabular}




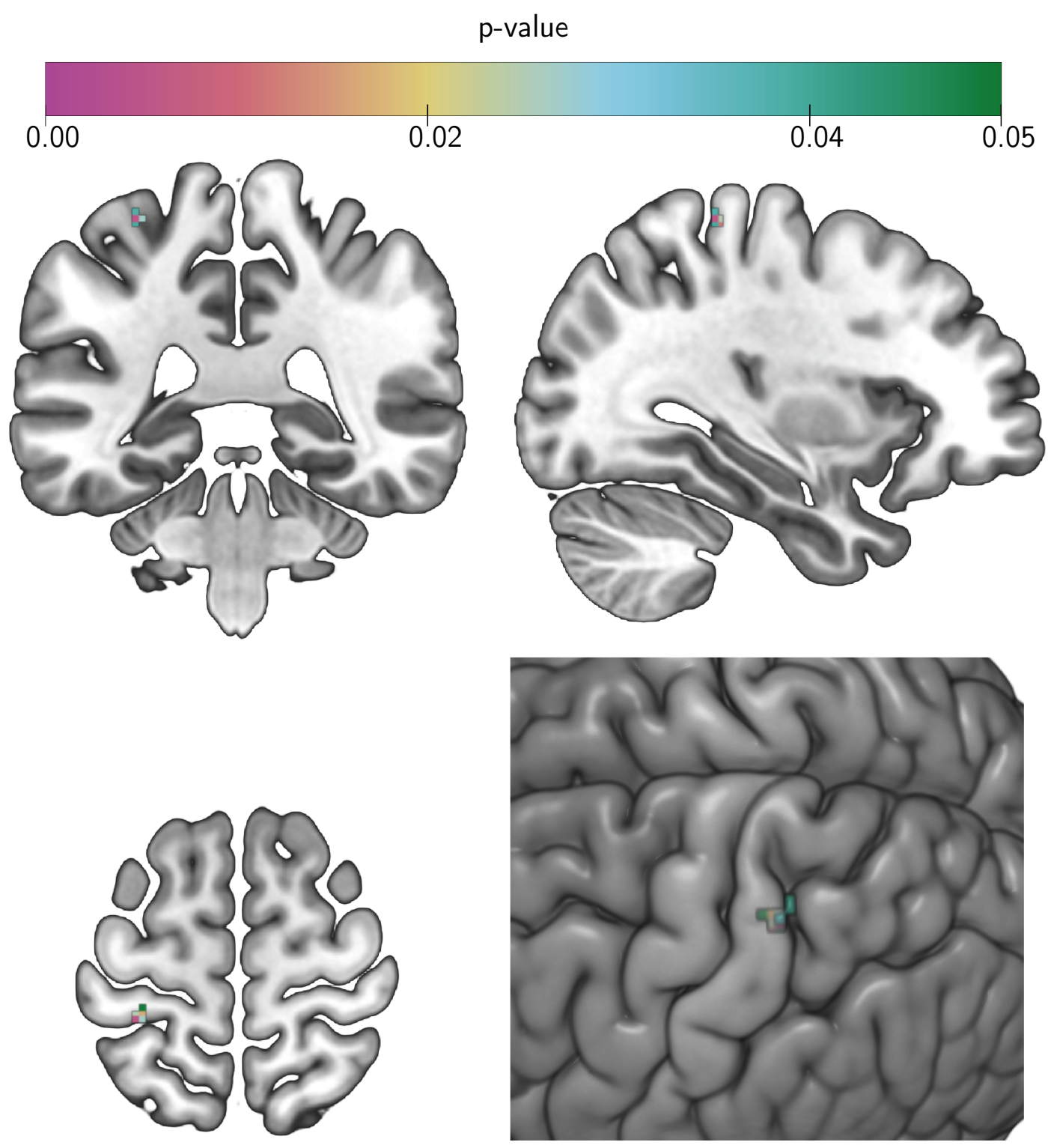

Figure 2.8.: Interaction effect grasp type $\times$ orientation Location of the significant voxels (thresholded with $\mathrm{p}=0.05$, voxel wise corrected for multiple comparison by permutation analysis with 100000 permutations) within the MNI152 standard brain template. Coloration indicates the $\mathrm{p}$-value of the voxel according to the displayed color bar. Axial and coronal slices use neurological convention (left on the left). 
p-value

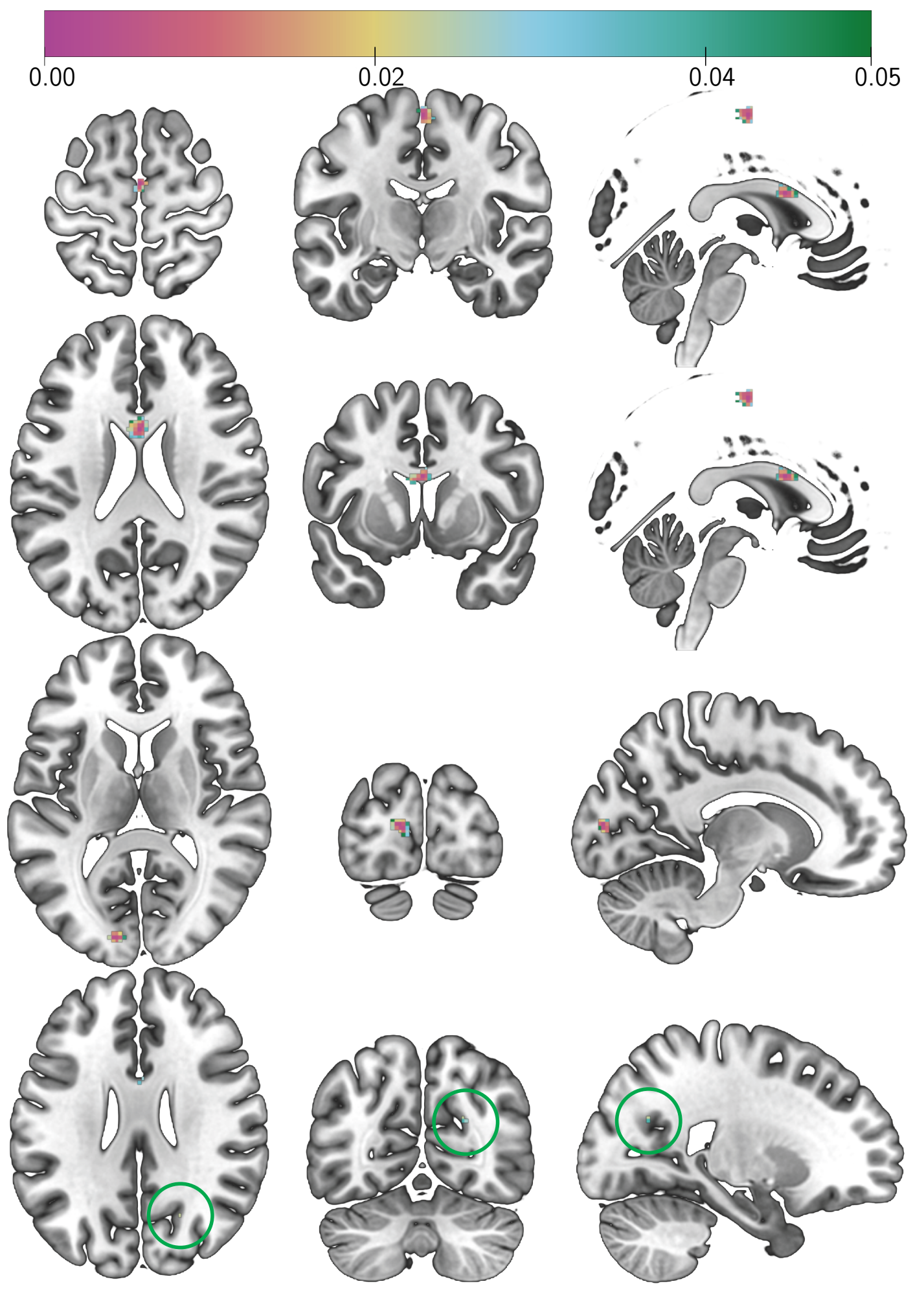

Figure 2.9.: Interaction effect grasp orientation $\times$ predictability See next page. 
Figure 2.9.: Continued from previous page. Interaction effect grasp orientation $\times$ predictability Location of the significant voxels (thresholded with $p=0.05$, voxel wise corrected for multiple comparison by permutation analysis with 100000 permutations) within the MNI152 standard brain template. Each row of sections is cut through the coordinates of the peak p-value of one cluster (see table 2.7 on page 85, clusters in the same order, top to bottom, as in the table). Coloration indicates the p-value of the voxel according to the displayed color bar. Axial and coronal slices use neurological convention (left on the left). Green circles highlight positions of small clusters.

\section{Reduced interaction grasp type $\times$ predictability}

This contrast showed no significant voxels.

\section{Reduced interaction grasp type $\times$ orientation $\times$ predictability}

This contrast showed no significant voxels.

\section{Main effect visual vs. auditory}

This contrast produced 10 clusters of significant voxels and three individual significant voxels (see table 2.8 on page 90 and figure 2.10 on the facing page). The lowest peak $\mathrm{p}$-value was in cluster mainly within the right hemisphere. By extension, this cluster is the second largest, with significant voxels in the superior temporal gyrus, middle temporal gyrus, Rolandic operculum, Heschl's gyrus, and supra marginal gyrus. The covered $\overline{B A} s$ are $22,21,42$, and 48 . The second cluster by peak p-value was the largest in this contrast. It was located on a comparable location but in the left hemisphere when compared to the first cluster. It also showed voxels within the superior temporal gyrus, middle temporal gyrus, Rolandic operculum, postcentral gyrus, and Heschl's gyrus. The involved BAs were 22, 48, 42, 41, and 21 . The third lowest peak p-value was within a cluster along the left superior temporal gyrus and Rolandic operculum $(B A ; 48,22,38$, and 21$)$. The fourth cluster was in the left middle occipital gyrus within $\mathrm{BA} \beta 19,18$, and 37 . The remaining clusters were small (under 20 voxels) and were within the left hemisphere along the middle occipital gyrus, superior parietal gyrus, and middle temporal gyrus (BAs $7,1819, \& 37$ ).

The individual significant voxels were all within the right hemisphere. Two were located in the middle occipital and the middle temporal gyrus, in $\mathrm{BA} \beta 7,18$, and 37. One located within the white matter below the middle occipital gyrus. 


$\longrightarrow$

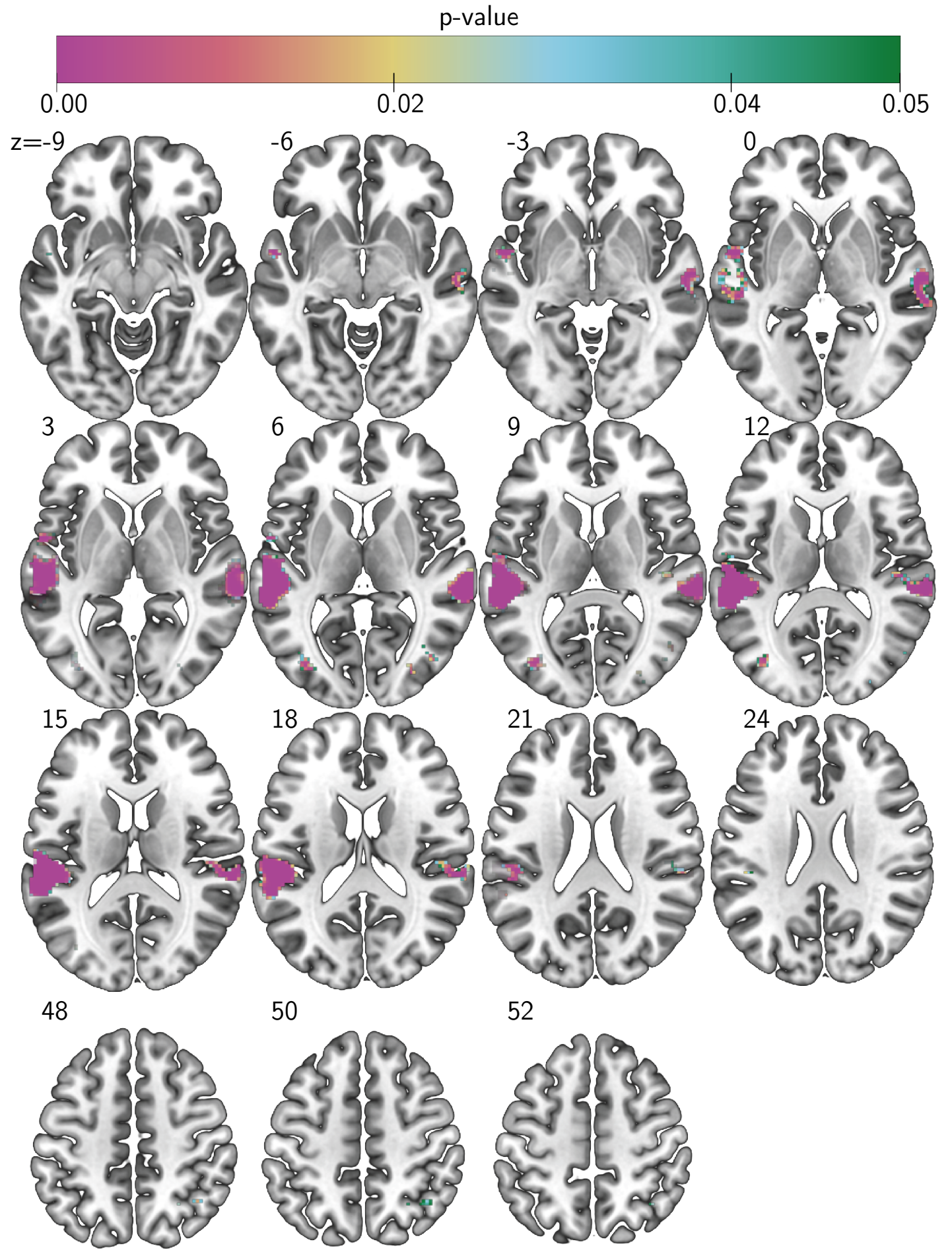

Figure 2.10.: Main effect visual vs. auditory. Location of the significant voxels (thresholded with $\mathrm{p}=0.05$, voxel wise corrected for multiple comparison by permutation analysis with 100000 permutations) within the MNI 152 standard brain template. Coloration indicates the p-value of the voxel according to the displayed color bar. Slices use neurological convention (left on the left). $Z$ values of the slices are stated above left of the respective slice. 
Table 2.8.: Main effect visual vs. auditory Location of the lowest p-value (voxel wise corrected for multiple comparison by permutation analysis with 100000 permutations) and size of surrounding cluster in voxels. The labels are sorted by proportion within the cluster, with the label of the peak location in italics.

\begin{tabular}{|c|c|c|c|c|c|}
\hline \multirow[t]{2}{*}{ Anatomical Area } & \multirow[t]{2}{*}{ Size } & \multicolumn{3}{|c|}{ Peak Coordinates } & \multirow[t]{2}{*}{$P_{\text {peak }}$} \\
\hline & & $X$ & Y & Z & \\
\hline $\begin{array}{l}\text { superior temporal gyrus, middle temporal } \\
\text { gyrus, Rolandic operculum, Heschl's gyrus, } \\
\text { supra marginal gyrus, (BA } 22,21,42,48)\end{array}$ & 679 & 64 & -30 & 2 & $<0.001$ \\
\hline superior temporal gyrus, & 1219 & -52 & -28 & 2 & $<0.001$ \\
\hline $\begin{array}{l}\text { superior temporal gyrus, middle temporal } \\
\text { gyrus, supra marginal gyrus, Rolandic Opercu- } \\
\text { lum, postcentral gyrus, Heschl's gyrus, } \mathrm{BA} 22 \text {, } \\
48,42,41,21)\end{array}$ & & -64 & -40 & 14 & $<0.001$ \\
\hline $\begin{array}{l}\text { superior temporal gyrus, Rolandic operculum, } \\
\text { superior temporal pole, }(\operatorname{BA} 48,22,38,21)\end{array}$ & 122 & -54 & 0 & 0 & $<0.001$ \\
\hline middle occipital gyrus, (BA $19,18,37)$ & 88 & -36 & -78 & 10 & $<0.001$ \\
\hline $\begin{array}{l}\text { white matter (middle occipital gyrus), middle } \\
\text { occipital gyrus, (BA } 18,19)\end{array}$ & 10 & 30 & -84 & 6 & 0.002 \\
\hline $\begin{array}{l}\text { middle occipital gyrus, middle temporal gyrus, } \\
(\operatorname{BA} 19,37)\end{array}$ & 8 & 44 & -76 & 6 & 0.007 \\
\hline middle temporal gyrus, $(\mathrm{BA} 19,37)$ & 4 & 50 & -68 & 10 & 0.009 \\
\hline $\begin{array}{l}\text { angular gyrus, inferior parietal gyrus, superior } \\
\text { parietal gyrus, }(\overline{\mathrm{BA}} 37)\end{array}$ & 12 & 40 & -56 & 48 & 0.016 \\
\hline middle occipital gyrus, (BA 18) & 1 & 28 & -92 & 14 & 0.025 \\
\hline superior parietal gyrus, $(\overrightarrow{\mathrm{BA}} 7)$ & 2 & 28 & -58 & 48 & 0.026 \\
\hline middle occipital gyrus, ( $\mathrm{BA} 18)$ & 2 & 30 & -90 & 12 & 0.034 \\
\hline middle temporal gyrus, $\sqrt{\mathrm{BA}} 37$ ) & 1 & 52 & -72 & 12 & 0.039 \\
\hline white matter (middle occipital gyrus) & 1 & 38 & -68 & 6 & 0.045 \\
\hline
\end{tabular}

\subsubsection{Discussion}

In this study we utilized modern encoding methods to discern the neural differences between predictable and unpredictable go-cues in a motor execution paradigm. Within this motor paradigm, we investigated the differences between grasp execution and wrist rotation as a proxy for reach processes. In the following section, we discuss our findings within the context of previous publications. 


\section{Grasp type and orientation encoding}

The orientation contrast produces only one cluster with the peak in the postcentral gyrus, but mainly located within the supraparietal lobule, in a region better known as intraparietal sulcus. This location has been observed in reach processing in a range of different studies (Macaluso et al., 2007; Cavina-Pratesi et al., 2010b; Bernier et al., 2017), giving support to our hypothesis that wrist rotation is part of the reach process.

The largest cluster in the grasp type analysis is over the hand area of the primary motor and sensory cortex (Penfield and Rasmussen, 1950, Schieber, 1999; O. Simon et al., 2002, Pimentel et al., 2011). Its involvement in grasping (Cavina-Pratesi et al., 2007, 2010b; Fiehler et al., 2011; Fabbri et al., 2014) and more specialized actions of the right hand (S. R. Simon et al., 2002. Milner et al., 2007; Horovitz et al., 2013; Ogawa and Imai, 2016, Rallis et al., 2018) has been well documented. It is noteworthy, that we do not see encoding for the reach process in our experiment, but involvement of this area in reach is also well documented (Prado et al., 2005 Filimon et al., 2007; Blangero et al., 2009). It is likely that the stronger effect of grasp encoding did overlay a reach encoding in this area.

The second cluster is more inferior to the first one and located in an area where other studies found representation for individual finger movements (Stoeckel et al., 2007. Nambu et al., 2015) and motor imagery (MI) of playing the piano (Meister et al., 2004). We hypothesise that this area is responsible for the difference in the finger action for the different grasps.

The smallest cluster in the grasp type analysis is located within the primary motor cortex as well, at a location which has been associated with different grasps execution (Begliomini et al., 2007a; Kuhtz-Buschbeck et al., 2008). Different studies have located this area either as a general hand area (Koeneke et al., 2004 I. G. Meister et al., 2009, Yuan et al., 2011) or associated it with finger movements (Jäncke et al., 2000; Koski et al., 2002, Post et al., 2007; Amiez et al., 2012, Cunningham et al., 2013). Most notably, Horovitz et al. (2013) found activity in this area, when subjects were writing with the right hand, a motor action involving fine finger and grasp control.

In the interaction contrast grasp type $x$ orientation, we observe one cluster of significant voxels in the postcentral gyrus. This location has been observed in other studies in a range of hand motor activity. Ranging from individual finger representation in both active, passive and MI context (Cunnington et al., 2006. Post et al., 2007; Cunningham et al., 2013: Dueñas et al., 2018), to wrist representation 
(Romaiguère et al., 2003; Naito et al., 2005; Suminski et al., 2007) and playing a keyboard instrument (Meister et al., 2005). This area is probably providing sensory feedback during grasp and reach processes. This is supported by the work of Gentile et al. (2011), who found visual and tactile feedback related to the hand of the subject in this location.

\section{Predictability in the brain}

The reduced main effect predictability shows a wide range of clusters, while the reduced interaction effect grasp orientation $\times$ predictability only has four clusters of significant voxels. All other interaction contrasts are empty, which shows that we achieved a good separation between the two processes we aimed to investigate. This is also supported by the movement time analysis.

Considering the predictability contrast, the cluster presenting the lowest peak pvalue in the right superior temporal gyrus is located in the posterior end of the lateral sulcus. This area shows activation during multisensory processing as well as motor execution in a multimodal reaction time experiment (Kansaku et al., 2004), indicating a central role in the initiation of motor actions in response to an unspecific external stimulus. It also shows activation during immediate execution vs. delayed recall of a finger tap patterns (Langner et al., 2013) and when subjects were asked to tap along to a rhythm (J. L. Chen et al., 2008). Milner et al. (2007) reported activation in the same area when subjects had to perform a complex manipulation task (balancing a top-heavy ruler) vs. simple manipulation (squeezing a rubber ball). This strengthens the interpretation that this area is involved in the temporal initiation of motor actions, since complex manipulation requires the temporal initiation of muscle sequences in a consecutive manner.

The largest cluster is covering the post- and precentral gyrus at the height of the middle frontal gyrus and extending into said gyrus. This part of the motor and somatosensory cortex is at the border between the mouth and and hand motor cortex (Meier et al., 2008). Since the motor cortex shows high inter-subject variability (Rao et al., 1995 Meier et al., 2008), a clear interpretation is difficult. The mouth motor cortex could have been involved due to subjects overtly counting the predictor stimuli (Hinton et al., 2004). This interpretation is supported by Wolfensteller et al. (2007), who found activation at a similar location when investigating mouth-related over armrelated activity. Matsuo et al. (2003) observed activity at this location when subjects were not allowed to move their fingers when tasked with counting the strokes in Japanese characters over when finger movements were permitted. 
However, there is an alternative explanation. Ullén et al. (2003) observed activity in this part of the motor cortex, when subjects had to tap different rhythms with each hand as compared to synchronous bimanual tapping. Horovitz et al. (2013) observed activity associated with writing, which requires a strong temporal coordination between the finger and hand muscles. While Eickhoff et al. (2011) showed dynamic adaptation of response bias in a direction detection task with changing left-right bias. It has also been shown to activate if subjects are required to move their hand and foot either in phase or out of phase (Rocca et al., 2007). This can be interpreted as the hand motor cortex recruiting surrounding areas during complex tasks. This is often an effect missed in typical motor cortex mapping studies, when the movement is limited to simple finger or wrist movements (Rao et al., 1995 Meier et al., 2008).

Our experiment unfortunately does not enable us to exclude the overt counting hypothesis. A follow-up experiment with a continuous and abstract timing cue could help to differentiate between both interpretations of primary motor cortex results.

The extension of this cluster to the middle frontal gyrus is easier to interpret. This area has been described as the human premotor cortex (for review see Schubotz and D. von Cramon, 2003). It has been associated with rule selection activity in tasks with either button press (Rowe et al., 2008) or shape selection on a 2D display via joystick (Lau et al., 2004). An involvement in the coordination of movement in time was shown by Lotze et al. (2003) who found stronger activity during rehearsal in amateur than professional musicians. More evidence for a temporal function of this premotor area is its involvement in a time order judgement task (Davis et al., 2009). It also shows activity during a language-related task, which has a temporal component, in judging the temporal order in a sentence ( $Y e$ et al., 2012). Another line of evidence comes from the examination of neurological disorders. When comparing the activation in early stage $\mathrm{PD}$ patients during pantomiming tool use, we see a stronger activation when the subject is off medication than on (Matt et al., 2017), indicating a stronger effort to produce such complex behaviour, possibly to compensate for the subthreshold initiation inhibition in early $\mathrm{PD}$. Another neuropathology associated with timing errors is schizophrenia. Patients show a difference in activation in this area during an auditory time discrimination task in comparison to healthy controls (Davalos et al., 2011).

Of particular interest is the fourth largest cluster in the predictability contrast. The cluster ranges from the SMA to the left middle central gyrus. The part of the SMA where we find significant voxels has mainly been attributed with bimanual control (Foltys et al., 2003; Debaere et al., 2004 Klöppel et al., 2007; Marchand et al., 2007; Grefkes et al., 2008; Müller et al., 2009; Gallivan et al., 2013a). However, 
the presence of activity during coordination between hand and arm movements hints at a more broader control function (Swinnen et al., 2010, Nakagawa et al., 2016). It also shows activation during more complex tasks such as pantomiming tool use (Rumiati et al., 2004; Ogawa and Imai, 2016), playing the piano (Meister et al., 2004), singing (Kleber et al., 2010), writing (Horovitz et al., 2013), or fluent speech production (Alario et al., 2006). On a more general view, SMA is involved in rule selection (M. Rushworth et al., 2002. Chouinard and Goodale, 2009. Schulte et al., 2009), movement planning and recall (Jankowski et al., 2009), and learning of complex movement sequences (Fernández-Seara et al., 2009).

There is strong evidence for SMA s involvement in temporal processing (for a meta-analysis see Schwartze et al., 2012). At the location of our significant voxels, other studies found activity when continuing to tap an auditory or visual given rhythm (Lewis et al., 2004), retrieving previously learned rhythmic patterns (Konoike et al., 2012) and during listening to a rhythm (Bengtsson et al., 2009).

For the interpretation of this cluster within our experiment, the work of FrançoisBrosseau et al. (2009) is of particular interest. The authors found activity within and close to our significant voxels during externally triggered and self-initiated finger movement. Our results in the SMA support this. Because the executed movement only involves one hand and is non-rhythmical executed, we conclude that the role of this area of the SMA is the timely initiation of the movement.

The pre-SMA has been described as part of the task salience (Seeley et al., 2007) or task active network (Buckner et al., 2009). Multiple studies found timing-related activity at the same area where we found predictability encoding in the cluster at the midline of said contrast, fifth by size and sixth by significance. In simple finger tapping or button press experiments, temporal dependent activity has been observed at this location (Dreher and Grafman, 2002, Riecker et al., 2003 Bengtsson et al., 2009). It is also activated during listening to simple rhythms or music (Bengtsson et al., 2009. Seger et al., 2013) or when counting sensory stimuli across modalities (Kansaku et al., 2006), which could explain our observed encoding.

Most importantly, the pre-SMA is activated when examining the subjective passing of time (Wittmann et al., 2010; Ortuño et al., 2011; Tipples et al., 2013), leading to the conclusion that the pre-SMA is involved in suprasecond somatosensory time durations (Schwartze et al., 2012). This is confirmed by our results.

However, Lau et al. (2004) observed activity in the same region as our experiment, when comparing free vs. routine or specified motor actions. This opens up the interesting idea that self-initiated and externally triggered movement initiation is a 
continuum rather then a categorical difference. Our experiment would be on this spectrum between completely free and completely forced motor initiation.

In the same location as our results within the ipsilateral SMA. Coull et al. (2015) found activity during temporal accumulation of auditory information. In our experiment, the subject had to accumulate the number of sensory inputs to prepare for the upcoming go-cue. Therefore, our experiment supports the finding of Coull et al. (2015).

We find significant voxels in the right superior frontal gyrus, in a region identified as the FEF in humans (Müri, 2006). Adam et al. (2003) observed activity in the same area during visual pre-cueing an upcoming finger tapping task. The obvious interpretation of this cluster is therefore the subject directing their visual attention towards the stimulus, probably in anticipation of the upcoming go-cue.

However, this part of the right premotor cortex has also been observed in a couple of other motor-related functions when engaging the right hand. Studies found activity during informative go-cues in a GoNoGo task (Jamadar et al., 2010), task switching (Cutini et al., 2008), finger tapping (Lehéricy et al., 2006) and Ml of finger tapping (Gao et al., 2011), object recognition through textile exploration (Reed et al., 2004), movement observation (Buccino et al., 2001; Jastorff et al., 2015), bimanual control (Koeneke et al., 2004, Rémy et al., 2008 Heitger et al., 2012 Solesio-Jofre et al., 2014), and playing a simplified PC tennis game (Modroño et al., 2013), all pointing to a much broader role of the ipsilateral premotor cortex in right hand movement control. The work of Kroeger et al. (2010) showed that the premotor cortex is inhibiting unwanted movements of the contralateral motor cortex. Mancini et al. (2009) showed an adaptation of the left premotor cortex during a paced hand flexion task. Therefore, we postulate a gating mechanism in the left premotor cortex for movements of the right hand by holding the motor cortex in a ready state like a loaded spring.

We find two clusters in the auditory cortex, one for each hemisphere. The left sided cluster is located in an area known for detection of temporal structure in auditory presented stimuli (Zatorre and Belin, 2001; Hove et al., 2013), predictive statistics (Giorgio et al., 2018), detecton of audiovisual synchrony (Lewis and Noppeney, 2010) and playing scales on a piano (Parsons et al., 2005). This area could be considered the location of rhythm in the human auditory cortex. It has a strong functional connection to the dorsal premotor cortex (J. L. Chen et al., 2008). This connection is probably employed when synchronizing motor execution to an external auditory timing signal.

The cluster on the right auditory cortex is in a comparable location and therefore 
probably has a similar function. It is activated when presenting complex auditory input, such as temporal structure of tonal sequences (Jamison et al., 2006) or processing music (Tillmann et al., 2003, Pfordresher et al., 2014, Toiviainen et al., 2014: Musso et al., 2015, Karpati et al., 2017). Foxe et al. (2002) described this area as responsible for audio somatosensory integration. This view has been substantiated by an increase in anatomical parameters such as cortical thickness and gray matter density in professional dancers (Karpati et al., 2017), as well as increased activity during a dance like joint action of the hands (Chauvigné et al., 2018). Wittmann et al. (2007) observed activity in the insula during a delayed discounting paradigm, indicating an involvement in time estimation, with Rekkas et al. (2005) describing an inhibitory BOLD response during a temporal order judgment task.

This cluster also extends superior into SII, where other studies observed activity during hand movement execution (Gerardin et al., 2000), auditory synchronous finger tapping (Aramaki et al., 2006), and when comparing active over passive finger movement (Mima et al., 1999). It is likely that our experiment, due to the employed alignment and smoothing, has mixed two separate information encoding clusters. It remains unclear why the ipsilateral SII appears in our results.

Another interesting cluster in the predictability contrast is on the left superior frontal gyrus. This area is also known as the PMd in humans (Schubotz and D. von Cramon, 2003). Activity in this part of the human PMd has been attributed to high order motor regulation, mainly inhibition of unwanted motor actions (Thoenissen et al., 2002. Lerner et al., 2009. Cai et al., 2013. Mohammadi et al., 2015), task switching (Braver et al., 2003), and judging the intention of observed motor actions (Buccino et al., 2007). Observed activity during the anticipation of stimuli (Badre et al., 2014 Yang et al., 2016), motor preparation (Thoenissen et al., 2002), temporal prediction (Coull et al., 2013) and synchronising to a auditory given pacing signal (Lewis et al., 2004) are of particular interest for our experiment. Our results further support the function of this part of the $\mathrm{PMd}$ to synchronize the initiation of motor actions with external stimuli. Together with the task switching property, we hypothesize the selectivity of appropriate motor actions based on the perceived state of the environment as the main function of this area.

The encoding in the right-side superior temporal gyrus, reaching into the insula and Heschl's gyrus, is probably an artifact of the auditory predictable go-cue. Activity here has appeared in other motor studies with auditory go-cues (Wenderoth et al., 2005; J. L. Chen et al., 2008 Kung et al., 2012. Pecenka et al., 2013).

Another cluster in our analysis is also probably caused by artifacts of the experimental design. Activity in the right middle occipital gyrus has been observed in a 
range of motor actions, when they are executed under visual guidance or feedback (Koeneke et al., 2004, Seidler et al., 2004; Poldrack et al., 2005. Higuchi et al., 2012. Floegel and Kell, 2017; Gardner et al., 2017), which is readily explained by encoding of visual attention (Giesbrecht et al., 2003; Newman et al., 2006, Ohlendorf et al., 2007), response to symbols (Carreiras et al., 2015), and temporal attention when visually cued (Coull et al., 2000).

The right superior parietal gyrus is part of the fronto-parietal attention network (Toro et al., 2008) and activity close to our significant voxels has been observed in a number of visuospatial attention and $\mathrm{Ml}$ tasks (Corbetta et al., 2005, Sturm et al., 2006. Tsubomi et al., 2009; Striemer et al., 2015: Zhang et al., 2016). Therefore, our first interpretation of the significant encoding is the increased attention of the subject to the task during the predictable trials.

However, reach-to grasp activity has also been observed (Chapman et al., 2002. Maratos et al., 2007), as well as hand orientation (Monaco et al., 2011), leaving the possibility that we have observed grasp related preparatory activity in this cluster.

We observe encoding in the right insula, which has been attributed with attention (Maguire et al., 2003; Clemens et al., 2011; Touroutoglou et al., 2012, L. J. Norman et al., 2017), inhibition of various motor actions (Maguire et al., 2003, Laurens et al., 2004 Leung and Cai, 2007, McNab et al., 2008, Chikazoe et al., 2009, Sebastian et al., 2012; Di Russo et al., 2016) and error monitoring (Ullsperger and von Cramon, 2003: Blasi et al., 2006 Klein et al., 2007; Grützmann et al., 2016). Although activity in this part of the insula has been observed in a number of experiments implementing a motor task, such as finger tapping (Bengtsson et al., 2004; Hanakawa et al., 2008) and right arm movements (Parkinson et al., 2009), the precise function in the motor initiation circuit is unclear. However, it seems to encode the temporal magnitude of a stimulus (Coull et al., 2015) and the temporal structure of complex movement sequences (Bengtsson et al., 2004). Dall'Acqua et al. (2018) observed increased activity in this part of the insula when the participant freely inhibited an action, contrary to when they were cued to inhibit. In light of this evidence, we postulate that the insula is responsible for the inhibition of unwanted movement initiation until the appropriate time point.

Activity in the right precentral gyrus has been observed in a number of studies involving right hand motor execution (Jäncke et al., 2000, Bijsterbosch et al., 2010. Plata Bello et al., 2015). Pouthas et al. (2005) observed activity at the same location as our results when subjects had to judge long time delays (around $1300 \mathrm{~ms}$ ) over short delays (around $450 \mathrm{~ms}$ ), Jäncke et al. (2000) during synchronization of finger tapping to a visual pacing signal and Bischoff et al. (2014) for judging the 
trajectory of the ball of a stylised tennis player serving. Other experiments showing activity involved uncertainty during motor execution (Sakai et al., 2000), memory and prediction during motor adaptation (Scheidt et al., 2012), as well as adjusting tapping to noticeable and unnoticeable disturbed auditory timing signals (Bijsterbosch et al., 2010). These studies suggest that this location is involved in the audio-visual integration of timing signals in motor execution for the right hand.

In the right superior temporal gyrus and Rolandic operculum, other studies found activity during the audio-visual cross modal stimulation (Lehmann et al., 2006), more explicitly during the onset of visual and auditory stimulus (Werner and Noppeney, 2011) and for finger tapping (Kornysheva and Schubotz, 2011). In our experiment, it is probably engaged during both pacing signals and functions as a low-level processing area for the presented pacing stimulus.

A small cluster in the frontal pole of the right superior frontal gyrus is of particular interest. This location has been observed in experiments investigating motor directed attention (Binkofski et al., 2002) and successful inhibition in a stop signal task (Ray Li et al., 2006). This suggests a high order planning function.

We also find significant encoding in the cerebellum at a location that has been observed in other experiments regarding hand actions (Haslinger et al., 2004 Seidler et al., 2004). The importance of the cerebellum in motor control is well known (for review see Manto et al., 2012). Its role in the initiation of movement, however, is strongly debated (Thach, 2014).

Our most superior cluster is located in the left postcentral gyrus, extending to the superior frontal and precentral gyrus. This location has been associated with the retrieval and execution control of complex finger movements (Hummel et al., 2004).

\section{Predictability interaction effects}

We only get significant results in the interaction grasp orientation $\times$ predictability. The largest cluster is covering the SMA close to the main effect predictability results. This area of the SMA has been described as the hand encoding subsection (Amiez and Petrides, 2014). Neural activity at this location has been associated with wrist movement (Szameitat et al., 2012), moving a joystick (Wenderoth et al., 2005), complex finger tapping paradigms (Coynel et al., 2010, Orban et al., 2010), and finger force response (Jackson et al., 2008). Schwartze et al. (2012) described this posterior part of the SMA as encoding sequential movements. This explains the orientation encoding, as this action required the sequence of wrist rotation $\rightarrow$ grasp $\rightarrow$ counter rotation. 
The encoding in the left superior occipital gyrus is harder to interpret. This area has been associated with visual working memory (Nenert et al., 2014; Wing et al., 2015), attention (Yamagishi et al., 2005) auditory spatial orientation (Wu et al., 2007), and hand-eye coordination (Lavrysen et al., 2008). Karabanov et al. (2009) observed activity during execution of both visual and auditory trained rhythms, leaving the precise function of this area during the course of our study unresolved.

The largest cluster in said contrast covers a part of the corpus callosum. A BOLD signal increase in this part of the callosum has been observed during tasks with increased load between hemispheres such as the strobe task (Zoccatelli et al., 2010), conflict processing (lannaccone et al., 2015), progress monitoring (Benn et al., 2014), and tracking of multiple objects (Atmaca et al., 2013). Most interestingly are the results of Bartolo et al. (2014), who observed activity in the corpus callosum when subjects were asked to judge if an visual presented object is within reachable distance or not. In light of those previous works, our results show a strong need for cross hemisphere integration in time critical reach processes, probably caused by cross hemispherical visual and motor integration.

\section{Limitations of the study}

The visual vs. auditory main effect shows two overlaps with the predictability contrast. Both are in the left and right auditory cortex. It is possible that the auditory condition shows up in the predictability contrast. The subjects reported consistently, that the auditory condition was easier than the visual condition. Therefore, the patterns of the auditory condition are probably stronger in the predictability condition than the visual patterns. This leads us to caution against interpreting the clusters in the predictability contrast surrounding the clusters in the auditory vs visual contrast (see figure 2.11 on the next page).

This study has a sizeable gender imbalance. Of the 14 subjects included in the analysis, only one was male. It has been observed that women also recruit the ipsilateral hemisphere in motor execution, while male subjects recruit more subcortical structures (Lissek et al., 2007). This could explain the extensive ipsilateral results in our study. This gender difference in the motor representation is an interesting topic for further studies.

We observed a number of clusters, that have been attributed with rhythm and overt counting. This is caused by the discrete and rhythmic nature of our timing signal. A new study would benefit from a continuous timing signal. An idea for a visual and continuous timing cue would be a circle with a shrinking diameter approaching 

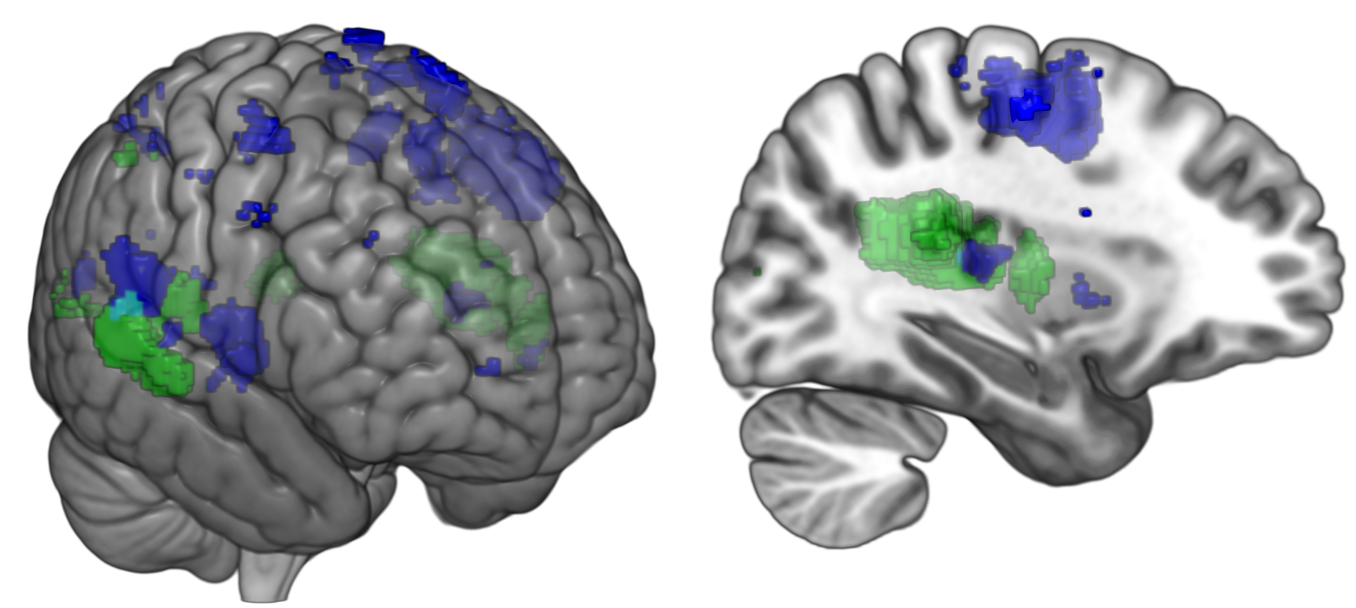

Figure 2.11.: Overlap between predictability contrast (blue) and the visual vs auditory contrast (green). Left panel showing the overlay in the right auditory cortex. To better visualize the surrounding clusters, the brain is viewed from the front right side direction. Right panel shows the overlap in the left auditory cortex. The view is from the inside of the brain slightly above the mid line pointing outwards.

the fixation dot and the subject executing the grasp, when the circle merges with the dot. A similar auditory paradigm is harder to design. A tone could either be varied in pitch, duration or volume. We used duration as the condition that is easy to judge by the subject. Pitch and volume perception are very subjective. Therefore, an experimental paradigm, requiring the subject to respond when a certain pitch or volume threshold is met, would be very unreliable.

\section{Conclusion}

In this study we successfully implemented a grasping paradigm incorporating two different grasps and wrist rotation conditions. We utilized go-cues with different predictability, one appearing without prior warning, and the other after either a visual or auditory pacing signal.

Regarding our first research question, as to which brain areas show significant encoding of the predictability condition, we mainly observed encoding of predictability in two brain areas previously implicated in motor initiation, the premotor cortex, cerebellum and most prominently the SMA. Of interest is the encoding in the frontal pole of the superior frontal gyrus $(\mathrm{BA} 10 \& 11)$ and the insula. Here the literature is sparse with respect to motor functions of those areas. 
The results in the premotor cortex are in line with electrophysiological recordings in macaques, where the PMd and AIP show significant population trajectories for RT differences (Michaels et al., 2015) and during movement preparation (Michaels et al., 2018).

It is noteworthy that we do not observe significant encoding in the hand area of the primary motor cortex (Rao et al., 1995; Lotze et al., 2000). Only surrounding primary motor areas, ranging from the face encoding areas on the inferior end and arm encoding areas at the superior end of the central gyrus show significant results. There are two possible explanations: either the motor cortex recruits surrounding areas when the movement has to be executed fast, or the movement information is stored in the surrounding areas of motor cortex during the predictable condition. Our experiment does not allow us to distinguish between both possibilities.

For our second research question, if the hand orientation is encoded separately form the grasp condition. We observed significant encoding of the wrist orientation in the supraparietal lobule, an area previously associated with reach processing (Macaluso et al., 2007; Cavina-Pratesi et al., 2010b: Bernier et al., 2017), while interaction with grasp is only observed in the primary sensory cortex. We therefore concluded that wrist rotation is part of the reach process and both are encoded in different processing streams, in line with the two stream hypothesis (Jeannerod, 1981).

Our third research question was, where do we observe grasp encoding in the human brain? We observe differences in the results between our experiment and previous research. Mainly we only find grasp encoding in the primary motor cortex. However, we also find significant voxels of the interaction effect grasp $\times$ orientation in the postcentral gyrus, implicating an interplay between grasp and wrist rotation. This could be explained by the difference in sensory feedback between the conditions and therefore does not contradict our interpretation.

\section{Outlook}

In our analysis, we ignored the delay period. A closer look at this time frame might give us insights into the encoding of the reach and grasp motor intention, giving us further insight into the separation between reach and grasp processing.

To increase SNR, we pooled over multiple time bins of the execution phase. An analysis of each time bin individually could give us a more time-resolved picture of the initiation process. Since we utilized a multiband sequence with increased time resolution, an analysis using dynamic causal modeling, Granger causality or transfer entropy could open up additional research questions (Granger, 1969, Schreiber, 2000. 
Friston et al., 2003), such as the entangling of information flow between the areas during the movement initiation process. 


\section{General Discussion}

The previous chapter described three projects investigating reach and grasp control in different contexts and with different methods. This chapter brings those works together, discusses the results in a combining framework and postulates new research questions.

\subsection{The reach and grasp networks revisited}

Both the first (see section 2.1 on page 20) and the third project (see section 2.3 on page 63) examine the separation between reaching and grasping: the first project by looking at the network properties in anesthetized macaques with resting state fMRI and the third one by examining the encoding of grasp and reach execution in behaving humans. In the third project, we used a wrist rotation as a minimal model for a reach. Both studies give us a clear indication of a separation between both networks: the resting state experiment by showing a significant clustering in the correlation matrix between the examined areas, perfectly along lines as the proposed network separation; the fMRI experiment in behaving humans by showing areas uniquely encoding the wrist rotation condition and others uniquely encoding grasp condition, with no overlap between both.

However, the results are not as clear cut on the second look. In the macaque project, significant interactions between areas belonging to different networks are found. The human study finds areas encoding the interaction between grasp and reach conditions. Although those areas are located in the sensory cortex in our analysis, they are also very close to areas associated with grasp processing in humans (compare to section 1.2.4 on page 7). Since the coregistration to the MNI template and the smoothing step introduce a spacial uncertainty of the reported location, those areas might be identical. Additionally, the used statistics are rather conservative and are known to suffer from reduced power with increasing subject number (see the bioRxiv prepublication of Hirose, 2020). Although Hirose (2020) proposes a more powerful analysis of information measurements, his work has not yet been peer- 
reviewed and we therefore used the more conservative statistics of Allefeld et al. (2016). It is possible that the resulting voxels seen in the third project are just the peaks of clusters, which could be revealed by a more powerful statistic.

A main point of contention is the separation of grasp and reach. Jeannerod (1984) defined the reach process as the arm transport until the anticipatory closing of the fingers and the grasp as the following manipulation of the grasped object. However, this separation underestimates the overlap between both processes. The closing of the fingers is depends on a range of parameters, both of the reach (Rand et al., 2006) as well as of the target object (Marteniuk et al., 1990), leaving the point of switch dependent on reach parameters. Before the fingers start to close, they already assume a shape suited to the object (Jeannerod, 1986, Santello and Soechting, 1998). This process is called preshaping and even takes place during memory-guided reaches (Winges et al., 2003). Therefore, the separation between transport and manipulation, as originally proposed by Jeannerod (1984), appears arbitrary.

The hand shapes during grasp can overwhelmingly be explained by a few degrees of freedom (Marco Santello, 1997; Mason et al., 2001), suggesting that the hand is controlled as a unit by the brain (Santello and Soechting, 1998). Separation of reach and grasp should therefore be considered more on an anatomical level, with the arm control as reach and the hand control as grasp. Project III investigated the affiliation of wrist rotation to either reach or grasp. Our results suggest the wrist rotation as a suitable separation boundary. However, the task design did not allow testing for other possible separations, such as wrist flexion. Therefore, further experiments to more precisely locate this boundary are needed.

Both reach and grasp processes are executed with a strong temporal synchronisation in human prehension (Jeannerod, 1984; Wallace and Weeks, 1988; Marteniuk et al., 1990; Paulignan et al., 1990. Gentilucci et al., 1992). This synchronicity is more caused by function than by neural control (Marteniuk et al., 1990), since they are independently corrected if either the reach or the grasp component is perturbed (Paulignan et al., 1990, Gentilucci et al., 1992. Saling et al., 1996). This functional coupling makes experimental design rather difficult and the results prone to become confounded between reach and grasp.

However, this coupling still does not explain the neural recordings in reach areas showing grasp-related activity (Galletti et al., 2003; Fattori et al., 2004, Raos et al., 2004; Stark et al., 2007; Fattori et al., 2009, 2010; Gamberini et al., 2011; Breveglieri et al., 2016), and vice versa (Kakei et al., 2001; Stark et al., 2007; Lehmann and Scherberger, 2013). Project I suggests predictive coding as an explanation for the 
observed neural activity. There is increasing evidence that the brain does not encode on a single neuron level, but utilises a population encoding (for review see Yuste, 2015). This has also been shown for the motor cortex of primates (Churchland et al., 2012; Intveld et al., 2018; Lara et al., 2018: Michaels et al., 2018) and humans (Pandarinath et al., 2015). Therefore, less importance should be placed on the correlation of individual neuron firing rates.

Only lesion studies have the power to prove that observed neural activity is necessary to execute a certain function (for review see Vaidya et al., 2019). This relation between observed activity and behaviour cannot be ascertained with other neurophysiological methods (Logothetis, 2008). There is only one lesion study showing support for reach and grasp not being processed in two different streams. Battaglini et al. (2002) showed defects in reach-to-grasp execution in macaques with a V6A lesion. When describing the defect, the authors state:

"The monkey rotated its hand abnormally, opening the grip laterally rather than downwards (frames $h-p$ ). This made grasping difficult and the duration of the trial was consequently prolonged. ...

In both monkeys, wrist rotation did not always match the orientation of the slit. Monkeys tended to orient the hand vertically, as if they had to pick up the slice of food from a vertical slit. When the slit was horizontal, monkeys were unable to appropriately rotate their wrist and often tried to force the piece of food outside the slit with their fingers." - Battaglini et al. (2002)

The described misgrasp was apparently caused by incorrect wrist rotation. V6A also encodes hand orientation during reach-to-grasp in macaques (Fattori et al., 2009. Gamberini et al., 2011), as does the human analogue, the superior parieto-occipital cortex (Cavina-Pratesi et al., 2010b; Monaco et al., 2011). Project III shows the wrist rotation to be part of the reach process. Therefore, the described lesions and neural activity are in line with the interpretation of V6A as a reach area (Fattori et al., 2001).

\subsection{Initiation of hand actions}

Aside from reach and grasp processing, project III (see section 2.3 on page 63) also examines the initiation of hand actions. The results are consistent with the literature regarding motor timing and motor initiation. Most notable is the observed significant encoding in the SMA premotor cortex and primary motor cortex. 
The SMA has been described as the location of the "when to act"-decision (Deecke, 1996) by realizing inhibition of M1 (Ball et al., 1999). There is also strong support for the SMA showing different neural activity between self-initiated and externally cued movements (Deiber et al., 1999; Jenkins et al., 2000; Cunnington et al., 2002). This supports the hypotheses that the difference between self-initiated actions and externally cued reactions is a continuum and not a categorical separation. The employed experimental paradigm in project III therefore allows the examination of the differences of the action-reaction continuum.

Although the SMA is primarily a motor-related area (for review see Picard and Strick, 1996), it also shows activity during timing judgment tasks regarding sensory input (for two meta-studies of the extensive neuroimaging corpus regarding the SMA see Wiener et al., 2010, Schwartze et al., 2012), leading to the conclusion that one of the roles of the SMA is mode independent time judgement. The presented experimental paradigm and its implementation for fMRI (project II in section 2.2 on page 40) opens up new possibilities in the research of $\mathrm{PD}$, attention deficit hyperactivity disorder (ADHD), and schizophrenia, which all show altered timing processing and motor initiation (for review see Meck and Benson, 2002). Collecting and comparing the decoding results from patients suffering from one of previous mentioned disorders would provide further insights into the disruption of motor initiation and time processing.

The role of the premotor cortex in movement initiation is much less clear. Activity in corresponding premotor areas has been observed in experiments concerning temporal processing (Gruber et al., 2000, Rao et al., 2001; Schubotz and D. Y. von Cramon, 2001; Lewis and Miall, 2002) and RT; in a delayed action task are predicable from neural activity in macaque F5 (Michaels et al., 2018). A well-supported function of the premotor cortex revolves around planning the action and facilitation of the primary motor cortex during execution (Umilta et al., 2007; Davare et al., 2008; Schaffelhofer and Scherberger, 2016. Michaels et al., 2018). It is therefore likely that the premotor cortex itself is not involved in the temporal judgment, but only receiving the temporal information from the SMA.

Differences in encoding in the BG was not observed in project III. This might be caused by a reduced sensitivity of the employed analysis method in subcortical areas. Jimura and Poldrack (2012) described lower sensitivity when using an MVPA decoding approach in subcortical areas. It is unclear, if that restriction also applies to an encoding approach. A univariate analysis of the dataset from project III is provided in appendix $B$ on page 177 .

With those in mind, we propose the "jack-in-the-box" model for motor initiation 
(see figure 3.1 on the following page). There the premotor cortex preloads the upcoming motor pattern and primes the primary motor cortex (the jack). Where the execution is blocked by the SMA and ipsitralateral (from the planned hand) primary motor cortex inhibiting the contralateral primary motor cortex (the loaded spring). The timing network in the SMA then starts to count up to the planned initiation by employing a timing circuit as proposed by Gibbon et al. (1984) (the crank). At the targeted point in time, the comparator switches, the inhibition from the primary motor cortex by the SMA is released (the lid pops) and the motor program is executed.

\subsection{Conclusion}

This thesis brought new insights into the separation of reach and grasp processing. It provided further support for the two-stream hypothesis as introduced by Jeannerod (1981). Functional connectivity in the macaque brain shows a clear separation between both networks. In the human brain, areas showing reach encoding are separate from grasp encoding areas. Possible explanations for the observation of neural activity across streams is provided.

A modular, 3D printed, fMRI compatible experimental set-up is presented. The modular design allows easy redesign and adjustment for new research questions. The design is first tested in a pilot study and subsequently in a full human fMRI experiment.

Additionally, initiation of prehension movements in humans was investigated. The results align strongly with research into the perception and reproduction of time. A new model is proposed for an initiation network of human prehension.

\subsection{Outlook}

Further questions regarding prehension control remain open, mainly the exact separation between the dorso-medial and dorso-ventral stream. This thesis proposes a separation at the wrist, but other possibilities are plausible.

Binkofski and Buxbaum (2013) draw the separation along grasp and use, not reach and grasp. Research into tool use often neglects the prehension component and only compares tools versus non-tools (for review see Reynaud et al., 2016). Umiltà et al. (2008) and Gallivan et al. (2013b) compare tool grasp with normal hand grasp in both humans and macaques and come to conflicting results. While Umiltà et al. 
Before initiation:

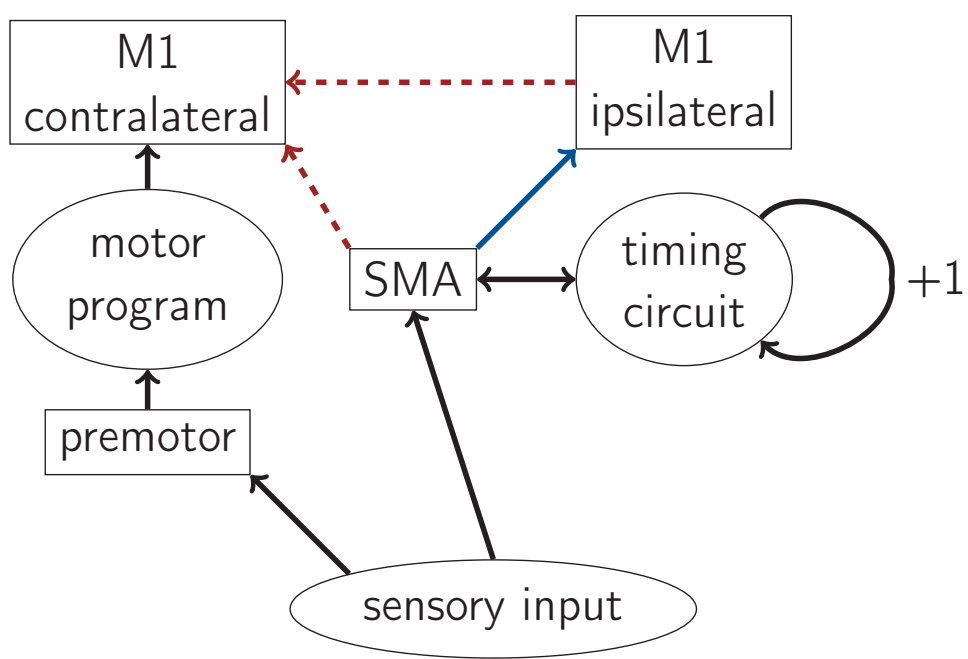

Initiation:

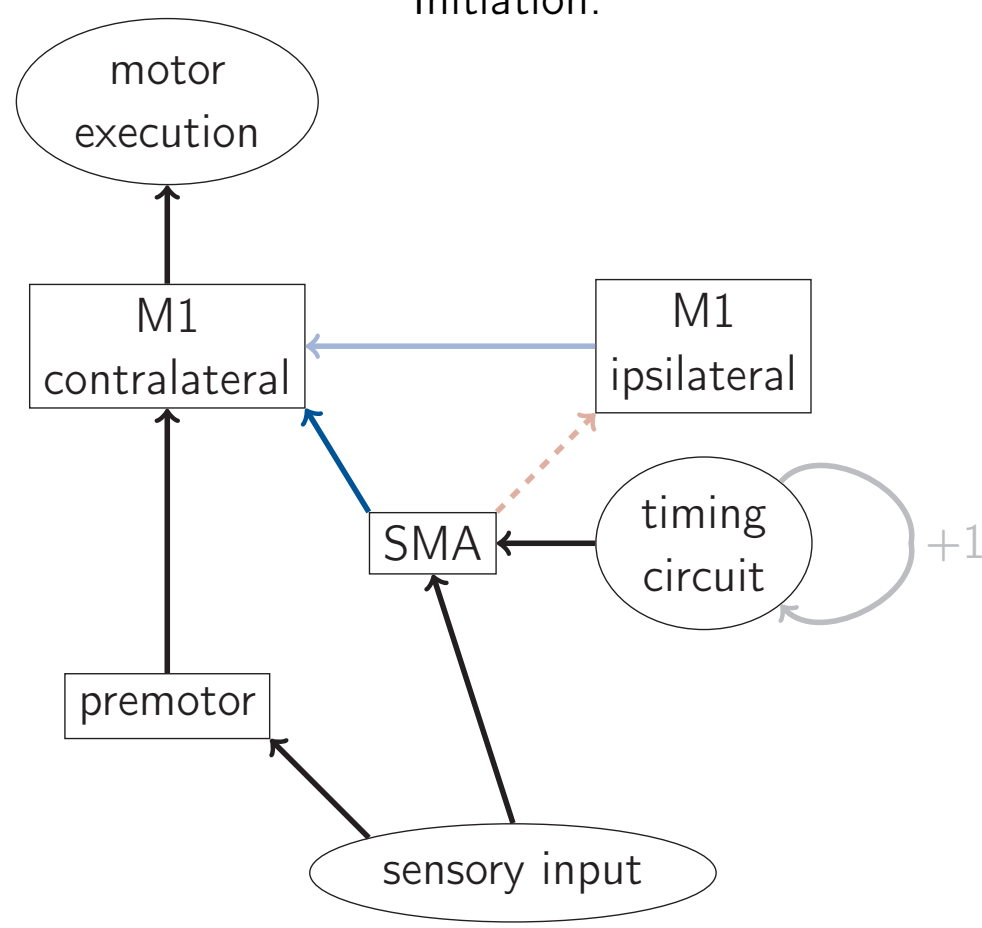

Figure 3.1.: Proposed model for motor initiation for prehension. Information flow and inhibition/facilitation between the cortical areas before the initiation (top) and at the time of initiation (bottom). Cortical areas are displayed in squares, information in ellipses. Black arrows show information transfer, red and dashed neural inhibition and blue facilitation. The shaded arrows in the bottom panel are hypothesized. 
(2008) show overlap between tool grasping and hand grasping in trained macaques, Gallivan et al. (2013b) showed that both use different networks in the human brain. Another line of separation can be drawn from the evolution of prehension. Iwaniuk and Whishaw (2000) questions the validity of combining a whole hand or power grasp with a finger precision grasp together as grasps. The argument the authors present is that reach and power grasps develop from use of the forelimbs for feeding behaviour and are the evolutionary older behaviour. This would put the separation between dorso-medial and dorso-ventral streams at the point of fine finger control and object manipulation.

Further experimentation into prehension in both humans and NHPs is needed to investigate these hypotheses. An experiment investigating fine finger movements with simple grasps could be designed as follows: An object with buttons for each finger is designed using the 3D printed parts proposed in project II section 2.2 on page 40). The subject has to reach for the object and grasp it, then either just hold it (baseline), press all buttons for 5 fingers (power grasp) or individual finger button presses in a instructed sequence (fine finger manipulation) and finally return the hand to the start position. 


\section{Bibliography}

Adam, J. J., W. Backes, J. Rijcken, P. Hofman, H. Kuipers, and J. Jolles (2003). "Rapid Visuomotor Preparation in the Human Brain: A Functional MRI Study". Cognitive Brain Research 16.1, pp. 1-10. DOI: 10.1016/S0926-6410(02)002045.

Alario, F.-X., H. Chainay, S. Lehericy, and L. Cohen (2006). "The Role of the Supplementary Motor Area (SMA) in Word Production". Brain Research 1076.1, pp. 129143. DOI: $10.1016 / \mathrm{j}$.brainres.2005.11.104.

Allefeld, C., K. Görgen, and J.-D. Haynes (2016). "Valid Population Inference for Information-Based Imaging: From the Second-Level t-Test to Prevalence Inference". Neurolmage 141, pp. 378-392. DOI: 10.1016/j . neuroimage.2016.07. 040

Allefeld, C. and J.-D. Haynes (2014). "Searchlight-Based Multi-Voxel Pattern Analysis of fMRI by Cross-Validated MANOVA". Neurolmage 89, pp. 345-357. DOI: 10.1016/j.neuroimage.2013.11.043.

Amiez, C., F. Hadj-Bouziane, and M. Petrides (2012). "Response Selection versus Feedback Analysis in Conditional Visuo-Motor Learning". Neurolmage 59.4, pp. 3723-3735. DOI: $10.1016 / \mathrm{j}$.neuroimage.2011.10.058.

Amiez, C. and M. Petrides (2014). "Neuroimaging Evidence of the Anatomo-Functional Organization of the Human Cingulate Motor Areas". Cerebral Cortex 24.3, pp. 563578. DOI: $10.1093 /$ cercor/bhs329.

Andersen, R. A., K. N. Andersen, E. J. Hwang, and M. Hauschild (2014). "Optic Ataxia: From Balint's Syndrome to the Parietal Reach Region". Neuron 81.5, pp. 967-983. DOI: 10.1016/j. neuron.2014.02.025.

Aramaki, Y., M. Honda, T. Okada, and N. Sadato (2006). "Neural Correlates of the Spontaneous Phase Transition during Bimanual Coordination". Cerebral Cortex 16.9, pp. 1338-1348. DOI: 10.1093/cercor/bhj075.

Astafiev, S. V., G. L. Shulman, C. M. Stanley, A. Z. Snyder, D. C. V. Essen, and M. Corbetta (2003). "Functional Organization of Human Intraparietal and Frontal Cortex for Attending, Looking, and Pointing". Journal of Neuroscience 23.11, pp. 4689-4699. DOI: 10.1523/JNEUROSCI .23-11-04689.2003 
Atmaca, S., W. Stadler, A. Keitel, D. V. M. Ott, J. Lepsien, and W. Prinz (2013). "Prediction Processes during Multiple Object Tracking (MOT): Involvement of Dorsal and Ventral Premotor Cortices". Brain and Behavior 3.6, pp. 683-700. DOI: $10.1002 / \mathrm{brb3} .180$.

Auerbach, S. H. and M. P. Alexander (1981). "Pure Agraphia and Unilateral Optic Ataxia Associated with a Left Superior Parietal Lobule Lesion". Journal of Neurology, Neurosurgery, and Psychiatry 44.5, pp. 430-432.

Badre, D., S. Lebrecht, D. Pagliaccio, N. M. Long, and J. M. Scimeca (2014). "Ventral Striatum and the Evaluation of Memory Retrieval Strategies". Journal of cognitive neuroscience 26.9, pp. 1928-1948. DOI: $10.1162 /$ jocn_a_00596

Bakola, S., M. Gamberini, L. Passarelli, P. Fattori, and C. Galletti (2010). "Cortical Connections of Parietal Field PEc in the Macaque: Linking Vision and Somatic Sensation for the Control of Limb Action". Cerebral Cortex 20.11, pp. 2592-2604. DOI: $10.1093 /$ cercor/bhq007.

Bakola, S., L. Passarelli, T. Huynh, D. Impieri, K. H. Worthy, P. Fattori, C. Galletti, K. J. Burman, and M. G. P. Rosa (2017). "Cortical Afferents and Myeloarchitecture Distinguish the Medial Intraparietal Area (MIP) from Neighboring Subdivisions of the Macaque Cortex". eNeuro 4.6. DOI: 10.1523/ENEURO.0344-17.2017.

Bálint, R. (1909). "Seelenlähmung Des "Schauens", Optische Ataxie, Räumliche Störung Der Aufmerksamkeit. Pp. 51-66". DOI: 10.1159/000210464.

Ball, T., A. Schreiber, B. Feige, M. Wagner, C. H. Lücking, and R. Kristeva-Feige (1999). "The Role of Higher-Order Motor Areas in Voluntary Movement as Revealed by High-Resolution EEG and fMRI". Neurolmage 10.6, pp. 682-694. DOI: 10.1006/nimg.1999.0507.

Bandettini, P. A., A. Jesmanowicz, E. C. Wong, and J. S. Hyde (1993). "Processing Strategies for Time-Course Data Sets in Functional Mri of the Human Brain". Magnetic Resonance in Medicine 30.2, pp. 161-173. DOI: 10.1002/mrm. 1910300204. Barch, D. M. and T. Yarkoni (2013). "Introduction to the Special Issue on Reliability and Replication in Cognitive and Affective Neuroscience Research". Cognitive, Affective, \& Behavioral Neuroscience 13.4, pp. 687-689. DOI: 10.3758/s13415013-0201-7.

Bartolo, A., Y. Coello, M. G. Edwards, S. Delepoulle, S. Endo, and A. M. Wing (2014). "Contribution of the Motor System to the Perception of Reachable Space: An fMRI Study". European Journal of Neuroscience 40.12, pp. 3807-3817. DOI: 10.1111/ejn.12742

Batista, A. P., G. Santhanam, B. M. Yu, S. I. Ryu, A. Afshar, and K. V. Shenoy (2007). "Reference Frames for Reach Planning in Macaque Dorsal Premotor Cor- 
tex". Journal of Neurophysiology 98.2, pp. 966-983. DOI: $10.1152 /$ jn.00421. 2006 .

Battaglia-Mayer, A. and R. Caminiti (2002). "Optic Ataxia as a Result of the Breakdown of the Global Tuning Fields of Parietal Neurones". Brain 125.2, pp. 225-237. DOI: $10.1093 /$ brain/awf034.

Battaglia-Mayer, A., S. Ferraina, A. Genovesio, B. Marconi, S. Squatrito, M. Molinari, F. Lacquaniti, and R. Caminiti (2001). "Eye-Hand Coordination during Reaching. II. An Analysis of the Relationships between Visuomanual Signals in Parietal Cortex and Parieto-Frontal Association Projections". Cerebral Cortex 11.6, pp. 528-544. DOI: $10.1093 /$ cercor/11.6.528.

Battaglia-Mayer, A., S. Ferraina, T. Mitsuda, B. Marconi, A. Genovesio, P. Onorati, F. Lacquaniti, and R. Caminiti (2000). "Early Coding of Reaching in the Parietooccipital Cortex". Journal of Neurophysiology 83.4, pp. 2374-2391. DOI: 10.1152/jn.2000.83.4.2374.

Battaglini, P. P., A. Muzur, C. Galletti, M. Skrap, A. Brovelli, and P. Fattori (2002). "Effects of Lesions to Area V6A in Monkeys". Experimental Brain Research 144.3, pp. 419-422. DOI: 10.1007/s00221-002-1099-4.

Baumann, M. A., M.-C. Fluet, and H. Scherberger (2009). "Context-Specific Grasp Movement Representation in the Macaque Anterior Intraparietal Area". Journal of Neuroscience 29.20, pp. 6436-6448. DOI: 10.1523/JNEUROSCI.5479-08.2009. Begliomini, C., A. Caria, W. Grodd, and U. Castiello (2007a). "Comparing Natural and Constrained Movements: New Insights into the Visuomotor Control of Grasping". PLOS ONE 2.10. DOI: 10.1371/journal.pone.0001108.

Begliomini, C., M. B. Wall, A. T. Smith, and U. Castiello (2007b). "Differential Cortical Activity for Precision and Whole-Hand Visually Guided Grasping in Humans". European Journal of Neuroscience 25.4, pp. 1245-1252. DOI: $10.1111 / \mathrm{j} .1460-$ 9568.2007.05365.x.

Bengtsson, S. L., H. H. Ehrsson, H. Forssberg, and F. Ullén (2004). "Dissociating Brain Regions Controlling the Temporal and Ordinal Structure of Learned Movement Sequences". European Journal of Neuroscience 19.9, pp. 2591-2602. DOI: 10.1111/j.0953-816X.2004.03269.x

Bengtsson, S. L., F. Ullén, H. Henrik Ehrsson, T. Hashimoto, T. Kito, E. Naito, H. Forssberg, and N. Sadato (2009). "Listening to Rhythms Activates Motor and Premotor Cortices". Cortex 45.1, pp. 62-71. DOI: 10.1016/j.cortex.2008.07. 002 . 
Benn, Y., T. L. Webb, B. P. I. Chang, Y.-H. Sun, I. D. Wilkinson, and T. F. D. Farrow (2014). "The Neural Basis of Monitoring Goal Progress". Frontiers in Human Neuroscience 8. DOI: $10.3389 /$ fnhum.2014.00688.

Bernier, P.-M., K. Whittingstall, and S. T. Grafton (2017). “Differential Recruitment of Parietal Cortex during Spatial and Non-Spatial Reach Planning". Frontiers in Human Neuroscience 11. DOI: 10.3389/fnhum.2017.00249.

Beurze, S. M., F. P. de Lange, I. Toni, and W. P. Medendorp (2009). "Spatial and Effector Processing in the Human Parietofrontal Network for Reaches and Saccades". Journal of Neurophysiology 101.6, pp. 3053-3062. DOI: 10.1152/jn. 91194.2008.

Bichsel, O., R. Gassert, L. Stieglitz, M. Uhl, H. Baumann-Vogel, D. Waldvogel, C. R. Baumann, and L. L. Imbach (2018). "Functionally Separated Networks for SelfPaced and Externally-Cued Motor Execution in Parkinson's Disease: Evidence from Deep Brain Recordings in Humans". Neurolmage 177, pp. 20-29. DOI: 10.1016/ j.neuroimage.2018.05.012.

Bijsterbosch, J. D., K.-H. Lee, M. D. Hunter, D. T. Tsoi, S. Lankappa, I. D. Wilkinson, A. T. Barker, and P. W. R. Woodruff (2010). "The Role of the Cerebellum in Sub- and Supraliminal Error Correction during Sensorimotor Synchronization: Evidence from fMRI and TMS". Journal of Cognitive Neuroscience 23.5, pp. 11001112. DOI: $10.1162 /$ jocn.2010.21506.

Binkofski, F., C. Dohle, S. Posse, K. M. Stephan, H. Hefter, R. J. Seitz, and H. J. Freund (1998). "Human Anterior Intraparietal Area Subserves Prehension: A Combined Lesion and Functional MRI Activation Study". Neurology 50.5, pp. 12531259. DOI: 10.1212/WNL.50.5.1253.

Binkofski, F., G. R. Fink, S. Geyer, G. Buccino, O. Gruber, N. J. Shah, J. G. Taylor, R. J. Seitz, K. Zilles, and H.-J. Freund (2002). "Neural Activity in Human Primary Motor Cortex Areas 4a and 4p Is Modulated Differentially by Attention to Action". Journal of Neurophysiology 88.1, pp. 514-519. DOI: 10.1152/jn.2002.88.1. 514.

Binkofski, F. and L. J. Buxbaum (2013). "Two Action Systems in the Human Brain". Brain and language 127.2, pp. 222-229. DOI: 10.1016/j.bandl.2012.07.007. Bischoff, M., K. Zentgraf, S. Pilgramm, R. Stark, B. Krüger, and J. Munzert (2014). "Anticipating Action Effects Recruits Audiovisual Movement Representations in the Ventral Premotor Cortex". Brain and Cognition 92, pp. 39-47. DOI: 10.1016/ j.bandc.2014.09.010. 
Blangero, A., M. M. Menz, A. McNamara, and F. Binkofski (2009). "Parietal Modules for Reaching". Neuropsychologia. Perception and Action 47.6, pp. 1500-1507. DOI: 10.1016/j.neuropsychologia.2008.11.030.

Blasi, G., T. E. Goldberg, T. Weickert, S. Das, P. Kohn, B. Zoltick, A. Bertolino, J. H. Callicott, D. R. Weinberger, and V. S. Mattay (2006). "Brain Regions Underlying Response Inhibition and Interference Monitoring and Suppression: Brain Regions Underlying Cognitive Control". European Journal of Neuroscience 23.6, pp. 16581664. DOI: $10.1111 / \mathrm{j} .1460-9568.2006 .04680 . x$.

Bono, M. G. D., C. Begliomini, U. Castiello, and M. Zorzi (2015). "Probing the Reaching-Grasping Network in Humans through Multivoxel Pattern Decoding". Brain and Behavior 5.11, e00412. DOI: 10.1002/brb3.412.

Borra, E., A. Belmalih, R. Calzavara, M. Gerbella, A. Murata, S. Rozzi, and G. Luppino (2008). "Cortical Connections of the Macaque Anterior Intraparietal (AIP) Area". Cerebral Cortex 18.5, pp. 1094-1111. DOI: 10.1093/cercor/bhm146.

Bosco, A., R. Breveglieri, D. Reser, C. Galletti, and P. Fattori (2015). "Multiple Representation of Reaching Space in the Medial Posterior Parietal Area V6A". Cerebral Cortex 25.6, pp. 1654-1667. DOI: 10.1093/cercor/bht420.

Bosco, A., R. Breveglieri, E. Chinellato, C. Galletti, and P. Fattori (2010). "Reaching Activity in the Medial Posterior Parietal Cortex of Monkeys Is Modulated by Visual Feedback". Journal of Neuroscience 30.44, pp. 14773-14785. DOI: 10 . 1523 / JNEUROSCI . 2313-10.2010.

Boudrias, M.-H., R. L. McPherson, S. B. Frost, and P. D. Cheney (2010). "Output Properties and Organization of the Forelimb Representation of Motor Areas on the Lateral Aspect of the Hemisphere in Rhesus Macaques". Cerebral Cortex 20.1, pp. 169-186. DOI: 10.1093/cercor/bhp084.

Boussaoud, D., J. Tanné-Gariépy, T. Wannier, and E. M. Rouiller (2005). "Callosal Connections of Dorsal versus Ventral Premotor Areas in the Macaque Monkey: A Multiple Retrograde Tracing Study". BMC Neuroscience 6.1, p. 67. DOI: 10 . 1186/1471-2202-6-67

Braver, T. S., J. R. Reynolds, and D. I. Donaldson (2003). "Neural Mechanisms of Transient and Sustained Cognitive Control during Task Switching". Neuron 39.4, pp. 713-726. DOI: 10.1016/S0896-6273(03)00466-5.

Bremner, L. R. and R. A. Andersen (2012). "Coding of the Reach Vector in Parietal Area 5d". Neuron 75.2, pp. 342-351. DOI: 10.1016/j.neuron.2012.03.041.

Brendel, A., R. S. Greulich, F. Niederman, and S. Trang (2020). "Towards a Greater Diversity of Replication Studies". AIS Transactions on Replication Research 6.1. DOI: 10.17705/1atrr.00063. 
Breveglieri, R., A. Bosco, C. Galletti, L. Passarelli, and P. Fattori (2016). "Neural Activity in the Medial Parietal Area V6A While Grasping with or without Visual Feedback". Scientific Reports 6. DOI: 10.1038/srep28893.

Breveglieri, R., C. Galletti, G. Dal Bò, K. Hadjidimitrakis, and P. Fattori (2013). "Multiple Aspects of Neural Activity during Reaching Preparation in the Medial Posterior Parietal Area V6A". Journal of Cognitive Neuroscience 26.4, pp. 878895. DOI: $10.1162 /$ jocn_a_00510.

Breveglieri, R., C. Galletti, S. Monaco, and P. Fattori (2008). "Visual, Somatosensory, and Bimodal Activities in the Macaque Parietal Area PEc". Cerebral Cortex 18.4, pp. 806-816. DOI: 10.1093/cercor/bhm127.

Brooks, R. A., J. H. Battocletti, A. Sances, S. J. Larson, R. L. Bowman, and V. Kudravcev (1975). "Nuclear Magnetic Relaxation in Blood". IEEE Transactions on Biomedical Engineering BME-22.1, pp. 12-18. DOI: 10.1109/TBME. 1975. 324533,

Brown, S. W. (1997). "Attentional Resources in Timing: Interference Effects in Concurrent Temporal and Nontemporal Working Memory Tasks". Perception \& Psychophysics 59.7, pp. 1118-1140. DOI: 10.3758/BF03205526.

Buccino, G., F. Binkofski, G. R. Fink, L. Fadiga, L. Fogassi, V. Gallese, R. J. Seitz, K. Zilles, G. Rizzolatti, and H.-J. Freund (2001). "Action Observation Activates Premotor and Parietal Areas in a Somatotopic Manner: An fMRI Study". European Journal of Neuroscience 13.2, pp. 400-404. DOI: 10.1111/j.1460-9568.2001. $01385 . x$

Buccino, G., A. Baumgaertner, L. Colle, C. Buechel, G. Rizzolatti, and F. Binkofski (2007). "The Neural Basis for Understanding Non-Intended Actions". Neurolmage. Cortical Control of Higher Motor Cognition 36, T119-T127. DOI: $10.1016 / \mathrm{j}$. neuroimage.2007.03.036.

Buckner, R. L., J. Sepulcre, T. Talukdar, F. M. Krienen, H. Liu, T. Hedden, J. R. Andrews-Hanna, R. A. Sperling, and K. A. Johnson (2009). "Cortical Hubs Revealed by Intrinsic Functional Connectivity: Mapping, Assessment of Stability, and Relation to Alzheimer's Disease". Journal of Neuroscience 29.6, pp. 1860-1873. DOI: $10.1523 /$ JNEUROSCI.5062-08.2009.

Buhusi, C. V. and W. H. Meck (2009). "Relative Time Sharing: New Findings and an Extension of the Resource Allocation Model of Temporal Processing". Philosophical Transactions of the Royal Society B: Biological Sciences 364.1525, pp. 1875-1885. DOI: $10.1098 /$ rstb. 2009.0022. 
Buneo, C. A., M. R. Jarvis, A. P. Batista, and R. A. Andersen (2002). "Direct Visuomotor Transformations for Reaching". Nature 416.6881, pp. 632-636. DOI: 10.1038/416632a.

Burle, B. and L. Casini (2001). "Dissociation between Activation and Attention Effects in Time Estimation: Implications for Internal Clock Models". Journal of Experimental Psychology. Human Perception and Performance 27.1, pp. 195-205. DOI: $10.1037 / / 0096-1523.27 .1 .195$.

Buxton, R. B. (2012). "Dynamic Models of BOLD Contrast". Neurolmage. 20 YEARS OF fMRI 62.2, pp. 953-961. DOI: 10.1016/j.neuroimage.2012.01.012.

Buxton, R. B., K. Uludag, D. J. Dubowitz, and T. T. Liu (2004). "Modeling the hemodynamic response to brain activation". Neuroimage 2004. DOI: $10.1016 / \mathrm{j}$. neuroimage.2004.07.013.

Buxton, R. B., E. C. Wong, and L. R. Frank (1998). "Dynamics of Blood Flow and Oxygenation Changes during Brain Activation: The Balloon Model". Magnetic Resonance in Medicine 39.6, pp. 855-864. DOI: 10.1002/mrm.1910390602.

Cai, W., C. J. Cannistraci, J. C. Gore, and H.-C. Leung (2013). "Sensorimotorindependent Prefrontal Activity during Response Inhibition". Human Brain Mapping 35.5, pp. 2119-2136. DOI: 10.1002/hbm. 22315

Caminiti, R., P. B. Johnson, C. Galli, S. Ferraina, and Y. Burnod (1991). "Making Arm Movements within Different Parts of Space: The Premotor and Motor Cortical Representation of a Coordinate System for Reaching to Visual Targets". Journal of Neuroscience 11.5, pp. 1182-1197.

Cappadocia, D. C., S. Monaco, Y. Chen, G. Blohm, and J. D. Crawford (2017). "Temporal Evolution of Target Representation, Movement Direction Planning, and Reach Execution in Occipital-Parietal-Frontal Cortex: An fMRI Study". Cerebral Cortex 27.11, pp. 5242-5260. DOI: 10.1093/cercor/bhw304.

Carlson, T. A., P. Schrater, and S. He (2003). "Patterns of Activity in the Categorical Representations of Objects". Journal of Cognitive Neuroscience 15.5, pp. 704-717. DOI: 10.1162 /jocn.2003.15.5.704.

Carreiras, M., I. Quiñones, J. A. Hernández-Cabrera, and J. A. Duñabeitia (2015). "Orthographic Coding: Brain Activation for Letters, Symbols, and Digits". Cerebral Cortex 25.12, pp. 4748-4760. DOI: 10.1093/cercor/bhu163.

Cavina-Pratesi, C., J. D. Connolly, S. Monaco, T. D. Figley, A. D. Milner, T. Schenk, and J. C. Culham (2018). "Human Neuroimaging Reveals the Subcomponents of Grasping, Reaching and Pointing Actions". Cortex. Where to Go Now with "What \& How" 98, pp. 128-148. DOI: 10.1016/j.cortex.2017.05.018. 
Cavina-Pratesi, C., M. A. Goodale, and J. C. Culham (2007). "FMRI Reveals a Dissociation between Grasping and Perceiving the Size of Real 3D Objects". PLoS ONE 2.5. DOI: $10.1371 /$ journal.pone.0000424

Cavina-Pratesi, C., M. letswaart, G. W. Humphreys, V. Lestou, and A. D. Milner (2010a). "Impaired Grasping in a Patient with Optic Ataxia: Primary Visuomotor Deficit or Secondary Consequence of Misreaching?" Neuropsychologia 48.1, pp. 226-234. DOI: 10.1016/j.neuropsychologia.2009.09.008.

Cavina-Pratesi, C., S. Monaco, P. Fattori, C. Galletti, T. D. McAdam, D. J. Quinlan, M. A. Goodale, and J. C. Culham (2010b). "Functional Magnetic Resonance Imaging Reveals the Neural Substrates of Arm Transport and Grip Formation in Reachto-Grasp Actions in Humans". The Journal of Neuroscience 30.31, pp. 1030610323. DOI: 10.1523/JNEUROSCI .2023-10.2010.

Chang, S. W. C. and L. H. Snyder (2012). "The Representations of Reach Endpoints in Posterior Parietal Cortex Depend on Which Hand Does the Reaching". Journal of Neurophysiology 107.9, pp. 2352-2365. DOI: 10.1152/jn.00852.2011.

Chapman, H., M. Gavrilescu, H. Wang, M. Kean, G. Egan, and U. Castiello (2002). "Posterior Parietal Cortex Control of Reach-to-Grasp Movements in Humans". European Journal of Neuroscience 15.12, pp. 2037-2042. DOI: $10.1046 /$ j.14609568.2002.02021.x.

Chauvigné, L. A. S., M. Belyk, and S. Brown (2018). "Taking Two to Tango: fMRI Analysis of Improvised Joint Action with Physical Contact". PLOS ONE 13.1. DOI: 10.1371/journal.pone.0191098.

Chen, J. E. and G. H. Glover (2015). "Functional Magnetic Resonance Imaging Methods". Neuropsychology Review 25.3, pp. 289-313. DOI: $10.1007 /$ s11065015-9294-9.

Chen, J. L., V. B. Penhune, and R. J. Zatorre (2008). "Listening to Musical Rhythms Recruits Motor Regions of the Brain". Cerebral Cortex 18.12, pp. 2844-2854. DOI: 10.1093/cercor/bhn042.

Chen, Y., S. Monaco, P. Byrne, X. Yan, D. Y. P. Henriques, and J. D. Crawford (2014). "Allocentric versus Egocentric Representation of Remembered Reach Targets in Human Cortex". Journal of Neuroscience 34.37, pp. 12515-12526. DOI: 10.1523/JNEUROSCI . 1445-14.2014.

Chikazoe, J., K. Jimura, S. Hirose, K.-i. Yamashita, Y. Miyashita, and S. Konishi (2009). "Preparation to Inhibit a Response Complements Response Inhibition during Performance of a Stop-Signal Task". The Journal of Neuroscience 29.50, pp. 15870-15877. DOI: $10.1523 /$ JNEUROSCI . 3645-09.2009. 
Chouinard, P. A. and M. A. Goodale (2009). "FMRI Adaptation during Performance of Learned Arbitrary Visuomotor Conditional Associations". Neurolmage 48.4, pp. 696-706. DOI: $10.1016 / j$.neuroimage.2009.07.020.

Christophel, T. B., P. lamshchinina, C. Yan, C. Allefeld, and J.-D. Haynes (2018). "Cortical Specialization for Attended versus Unattended Working Memory". Nature Neuroscience 21.4, p. 494. DOI: 10.1038/s41593-018-0094-4.

Churchland, M. M., J. P. Cunningham, M. T. Kaufman, J. D. Foster, P. Nuyujukian, S. I. Ryu, and K. V. Shenoy (2012). "Neural Population Dynamics during Reaching". Nature 487.7405, pp. 51-56. DOI: 10.1038/nature11129.

Churchland, M. M., G. Santhanam, and K. V. Shenoy (2006a). "Preparatory Activity in Premotor and Motor Cortex Reflects the Speed of the Upcoming Reach". Journal of Neurophysiology 96.6, pp. 3130-3146. DOI: 10.1152/jn.00307.2006.

Churchland, M. M., B. M. Yu, S. I. Ryu, G. Santhanam, and K. V. Shenoy (2006b). "Neural Variability in Premotor Cortex Provides a Signature of Motor Preparation". The Journal of Neuroscience: The Official Journal of the Society for Neuroscience 26.14, pp. 3697-3712. DOI: 10.1523/JNEUROSCI.3762-05.2006.

Cisek, P. (2006). "Integrated Neural Processes for Defining Potential Actions and Deciding between Them: A Computational Model". Journal of Neuroscience 26.38, pp. 9761-9770. DOI: 10.1523/JNEUROSCI.5605-05.2006.

Cisek, P. and J. F. Kalaska (2002). "Simultaneous Encoding of Multiple Potential Reach Directions in Dorsal Premotor Cortex". Journal of Neurophysiology 87.2, pp. 1149-1154. DOI: 10.1152/jn.00443.2001.

Clemens, B., M. Zvyagintsev, A. Sack, A. Heinecke, K. Willmes, and W. Sturm (2011). "Revealing the Functional Neuroanatomy of Intrinsic Alertness Using fMRI: Methodological Peculiarities". PLOS ONE 6.9. DOI: $10.1371 /$ journal .pone. 0025453 .

Cohen, Y. E. and R. A. Andersen (2002). "A Common Reference Frame for Movement Plans in the Posterior Parietal Cortex". Nature Reviews. Neuroscience 3.7, pp. 553562. DOI: $10.1038 / \mathrm{nrn} 873$.

Colby, C. L. and J.-R. Duhamel (1991). "Heterogeneity of Extrastriate Visual Areas and Multiple Parietal Areas in the Macaque Monkey". Neuropsychologia 29.6, pp. 517-537. DOI: 10.1016/0028-3932(91)90008-V.

Connolly, J. D., R. A. Andersen, and M. A. Goodale (2003). "FMRI Evidence for a 'parietal Reach Region' in the Human Brain". Experimental Brain Research 153.2, pp. 140-145. DOI: 10.1007/s00221-003-1587-1. 
Cook, R., G. Bird, C. Catmur, C. Press, and C. Heyes (2014). "Mirror Neurons: From Origin to Function." Behavioral and Brain Sciences 37.2, pp. 177-192. DOI: 10.1017/S0140525X13000903.

Corbetta, M., A. P. Tansy, C. M. Stanley, S. V. Astafiev, A. Z. Snyder, and G. L. Shulman (2005). "A Functional MRI Study of Preparatory Signals for Spatial Location and Objects". Neuropsychologia 43.14, pp. 2041-2056. DOI: $10.1016 / \mathrm{j}$. neuropsychologia.2005.03.020.

Coull, J. T., C. D. Frith, C. Büchel, and A. C. Nobre (2000). "Orienting Attention in Time: Behavioural and Neuroanatomical Distinction between Exogenous and Endogenous Shifts". Neuropsychologia 38.6, pp. 808-819. DOI: 10.1016/S00283932(99) 00132-3.

Coull, J. T., P. Charras, M. Donadieu, S. Droit-Volet, and F. Vidal (2015). "SMA Selectively Codes the Active Accumulation of Temporal, Not Spatial, Magnitude". Journal of Cognitive Neuroscience 27.11, pp. 2281-2298. DOI: $10.1162 /$ jocn_ a_00854.

Coull, J. T., K. Davranche, B. Nazarian, and F. Vidal (2013). "Functional Anatomy of Timing Differs for Production versus Prediction of Time Intervals". Neuropsychologia. Special Issue: How Does the Brain Process Time? 51.2, pp. 309-319. DOI: $10.1016 / \mathrm{j}$.neuropsychologia.2012.08.017.

Council, N. R. (2003). Guidelines for the Care and Use of Mammals in Neuroscience and Behavioral Research. DOI: 10.17226/10732.

Cox, D. D. and R. L. Savoy (2003). "Functional Magnetic Resonance Imaging (fMRI) "Brain Reading": Detecting and Classifying Distributed Patterns of fMRI Activity in Human Visual Cortex". Neurolmage 19.2, pp. 261-270. DOI: 10.1016/S10538119(03) 00049-1.

Coynel, D., G. Marrelec, V. Perlbarg, M. Pélégrini-lssac, P.-F. Van de Moortele, K. Ugurbil, J. Doyon, H. Benali, and S. Lehéricy (2010). "Dynamics of MotorRelated Functional Integration during Motor Sequence Learning". Neurolmage 49.1, pp. 759-766. DOI: $10.1016 / j$.neuroimage.2009.08.048.

Crammond, D. J. and J. F. Kalaska (1996). "Differential Relation of Discharge in Primary Motor Cortex and Premotor Cortex to Movements versus Actively Maintained Postures during a Reaching Task". Experimental brain research 108.1, pp. 45-61.

- (2000). "Prior Information in Motor and Premotor Cortex: Activity During the Delay Period and Effect on Pre-Movement Activity". Journal of Neurophysiology 84.2, pp. 986-1005. DOI: 10.1152/jn.2000.84.2.986.

Culham, J. C., C. Cavina-Pratesi, and A. Singhal (2006). "The Role of Parietal Cortex in Visuomotor Control: What Have We Learned from Neuroimaging?" Neuropsy- 
chologia. Visuomotor Functions of the Posterior Parietal Cortex 44.13, pp. 26682684. DOI: $10.1016 / j$.neuropsychologia.2005.11.003.

Culham, J. C., S. L. Danckert, J. F. X. D. Souza, J. S. Gati, R. S. Menon, and M. A. Goodale (2003). "Visually Guided Grasping Produces fMRI Activation in Dorsal but Not Ventral Stream Brain Areas". Experimental Brain Research 153.2, pp. 180-189. DOI: 10.1007/s00221-003-1591-5.

Culham, J. C. and K. F. Valyear (2006). "Human Parietal Cortex in Action". Current Opinion in Neurobiology. Cognitive Neuroscience 16.2, pp. 205-212. DOI: 10 . 1016/j.conb.2006.03.005.

Cunningham, D. A., A. Machado, G. H. Yue, J. R. Carey, and E. B. Plow (2013). "Functional Somatotopy Revealed across Multiple Cortical Regions Using a Model of Complex Motor Task". Brain research 1531, pp. 25-36. DOI: $10.1016 / \mathrm{j}$. brainres.2013.07.050.

Cunnington, R., C. Windischberger, L. Deecke, and E. Moser (2002). "The Preparation and Execution of Self-Initiated and Externally-Triggered Movement: A Study of Event-Related fMRl". Neurolmage 15.2, pp. 373-385. DOI: $10.1006 / \mathrm{nimg}$. 2001.0976 .

Cunnington, R., C. Windischberger, S. Robinson, and E. Moser (2006). "The Selection of Intended Actions and the Observation of Others' Actions: A Time-Resolved fMRI Study". Neurolmage 29.4, pp. 1294-1302. DOI: 10.1016/j . neuroimage. 2005.09.028.

Cutini, S., P. Scatturin, E. Menon, P. S. Bisiacchi, L. Gamberini, M. Zorzi, and R. Dell'Acqua (2008). "Selective Activation of the Superior Frontal Gyrus in TaskSwitching: An Event-Related fNIRS Study". Neurolmage 42.2, pp. 945-955. DOI: $10.1016 / j$. neuroimage.2008.05.013

Dall'Acqua, T., C. Begliomini, R. Motta, D. Miotto, and U. Castiello (2018). "Effects of Intentionality and Subliminal Information in Free-Choices to Inhibit". Neuropsychologia 109, pp. 28-38. DOI: 10.1016/j.neuropsychologia.2017.11.035.

Davalos, D. B., D. C. Rojas, and J. R. Tregellas (2011). "Temporal Processing in Schizophrenia: Effects of Task-Difficulty on Behavioral Discrimination and Neuronal Responses". Schizophrenia Research 127.1-3, pp. 123-130. DOI: 10.1016/ j.schres.2010.06.020.

Davare, M., R. Lemon, and E. Olivier (2008). "Selective Modulation of Interactions between Ventral Premotor Cortex and Primary Motor Cortex during Precision Grasping in Humans". The Journal of Physiology 586.Pt 11, pp. 2735-2742. DOI: 10.1113/jphysiol.2008.152603. 
Davare, M., J. C. Rothwell, and R. N. Lemon (2010). "Causal Connectivity between the Human Anterior Intraparietal Area and Premotor Cortex during Grasp". Current Biology 20.2, pp. 176-181. DOI: $10.1016 /$ j.cub.2009.11.063

Davare, M., A. Zénon, M. Desmurget, and E. Olivier (2015). “Dissociable Contribution of the Parietal and Frontal Cortex to Coding Movement Direction and Amplitude". Frontiers in Human Neuroscience 9. DOI: 10.3389/fnhum. 2015.00241.

Davis, B., J. Christie, and C. Rorden (2009). "Temporal Order Judgments Activate Temporal Parietal Junction". Journal of Neuroscience 29.10, pp. 3182-3188. DOI: 10.1523/JNEUROSCI.5793-08.2009.

Debaere, F., N. Wenderoth, S. Sunaert, P. Van Hecke, and S. Swinnen (2004). "Cerebellar and Premotor Function in Bimanual Coordination: Parametric Neural Responses to Spatiotemporal Complexity and Cycling Frequency". Neurolmage 21.4, pp. 1416-1427. DOI: 10.1016/j .neuroimage.2003.12.011.

Deecke, L. (1996). "Planning, Preparation, Execution, and Imagery of Volitional Action". Cognitive Brain Research. Mental Representations of Motor Acts 3.2, pp. 59-64. DOI: 10.1016/0926-6410(95)00046-1.

Deiber, M.-P., M. Honda, V. Ibañez, N. Sadato, and M. Hallett (1999). "Mesial Motor Areas in Self-Initiated Versus Externally Triggered Movements Examined With fMRI: Effect of Movement Type and Rate". Journal of Neurophysiology 81.6, pp. 3065-3077. DOI: 10.1152/jn.1999.81.6.3065.

Dempsey, M. F. and B. Condon (2001). "Thermal Injuries Associated with MRI". Clinical Radiology 56.6, pp. 457-465. DOI: 10.1053/crad.2000.0688

Dennis, A. and J. Valacich (2014). "A Replication Manifesto". AIS Transactions on Replication Research 1.1. DOI: 10.17705/1atrr.00001.

Desmurget, M. and S. Grafton (2000). "Forward Modeling Allows Feedback Control for Fast Reaching Movements". Trends in Cognitive Sciences 4.11, pp. 423-431. DOI: $10.1016 / \mathrm{s} 1364-6613(00) 01537-0$.

Di Pellegrino, G., L. Fadiga, L. Fogassi, V. Gallese, and G. Rizzolatti (1992). “Understanding Motor Events: A Neurophysiological Study". Experimental brain research 91.1, pp. 176-180.

Di Russo, F., G. Lucci, V. Sulpizio, M. Berchicci, D. Spinelli, S. Pitzalis, and G. Galati (2016). "Spatiotemporal Brain Mapping during Preparation, Perception, and Action". Neurolmage 126, pp. 1-14. DOI: 10.1016/j . neuroimage. 2015. 11.036 .

Diogo, R., B. G. Richmond, and B. Wood (2012). "Evolution and Homologies of Primate and Modern Human Hand and Forearm Muscles, with Notes on Thumb Movements and Tool Use". Journal of Human Evolution, p. 15. 
Donders, F. C. (1969). "On the Speed of Mental Processes". Acta Psychologica 30, pp. 412-431. DOI: 10.1016/0001-6918(69)90065-1.

Dreher, J.-C. and J. Grafman (2002). "The Roles of the Cerebellum and Basal Ganglia in Timing and Error Prediction: Cerebellar and Striatal Functions in Timing and Error Prediction". European Journal of Neuroscience 16.8, pp. 1609-1619. DOI: $10.1046 / j .1460-9568.2002 .02212 . x$.

Dueñas, J., J. Sulzer, P. Stämpfli, M.-C. Hepp-Reymond, S. Kollias, E. Seifritz, and R. Gassert (2018). "BOLD Signal in Sensorimotor Regions Reveals Differential Encoding of Passive Forefinger Velocity and Displacement Amplitude". Neurolmage 173, pp. 332-340. DOI: $10.1016 /$ j.neuroimage.2018.02.052.

Eickhoff, S. B., W. Pomjanski, O. Jakobs, K. Zilles, and R. Langner (2011). "Neural Correlates of Developing and Adapting Behavioral Biases in Speeded Choice Reactions-an fMRI Study on Predictive Motor Coding". Cerebral Cortex (New York, N.Y.: 1991) 21.5, pp. 1178-1191. DOI: 10.1093/cercor/bhq188.

Erlhagen, W. and G. Schöner (2002). "Dynamic Field Theory of Movement Preparation". Psychological Review 109.3, pp. 545-572. DOI: 10.1037/0033-295X. 109.3.545.

Etzel, J. A., J. M. Zacks, and T. S. Braver (2013). "Searchlight Analysis: Promise, Pitfalls, and Potential". Neurolmage 78, pp. 261-269. DOI: $10.1016 / \mathrm{j}$. neuroimage . 2013.03.041.

Fabbri, S., L. Strnad, A. Caramazza, and A. Lingnau (2014). "Overlapping Representations for Grip Type and Reach Direction". Neurolmage 94, pp. 138-146. DOI: 10.1016/j.neuroimage.2014.03.017

Faraci, F. M. and K. R. Breese (1993). "Nitric Oxide Mediates Vasodilatation in Response to Activation of N-Methyl-D-Aspartate Receptors in Brain." Circulation Research 72.2, pp. 476-480. DOI: 10.1161/01.RES.72.2.476.

Fattori, P., R. Breveglieri, K. Amoroso, and C. Galletti (2004). "Evidence for Both Reaching and Grasping Activity in the Medial Parieto-Occipital Cortex of the Macaque". European Journal of Neuroscience 20.9, pp. 2457-2466. DOI: 10 . 1111/j.1460-9568.2004.03697.x.

Fattori, P., R. Breveglieri, N. Marzocchi, D. Filippini, A. Bosco, and C. Galletti (2009). "Hand Orientation during Reach-to-Grasp Movements Modulates Neuronal Activity in the Medial Posterior Parietal Area V6A". Journal of Neuroscience 29.6, pp. 1928-1936. DOI: 10.1523/JNEUROSCI .4998-08.2009.

Fattori, P., M. Gamberini, D. F. Kutz, and C. Galletti (2001). '“Arm-Reaching' Neurons in the Parietal Area V6A of the Macaque Monkey". European Journal of Neuroscience 13.12, pp. 2309-2313. DOI: 10.1046/j.0953-816x.2001.01618.x. 
Fattori, P., D. F. Kutz, R. Breveglieri, N. Marzocchi, and C. Galletti (2005). "Spatial Tuning of Reaching Activity in the Medial Parieto-Occipital Cortex (Area V6A) of Macaque Monkey". European Journal of Neuroscience 22.4, pp. 956-972. DOI: 10.1111/j.1460-9568.2005.04288.x

Fattori, P., V. Raos, R. Breveglieri, A. Bosco, N. Marzocchi, and C. Galletti (2010). "The Dorsomedial Pathway Is Not Just for Reaching: Grasping Neurons in the Medial Parieto-Occipital Cortex of the Macaque Monkey". Journal of Neuroscience 30.1, pp. 342-349. DOI: 10.1523/JNEUROSCI. 3800-09.2010.

Faugier-Grimaud, S., C. Frenois, and D. G. Stein (1978). "Effects of Posterior Parietal Lesions on Visually Guided Behavior in Monkeys". Neuropsychologia 16.2, pp. 151168. DOI: $10.1016 / 0028-3932$ (78)90103-3

Fernández-Seara, M. A., M. Aznárez-Sanado, E. Mengual, F. R. Loayza, and M. A. Pastor (2009). "Continuous Performance of a Novel Motor Sequence Leads to Highly Correlated Striatal and Hippocampal Perfusion Increases". Neurolmage 47.4, pp. 1797-1808. DOI: 10.1016/j.neuroimage.2009.05.061.

Ferraina, S., P. B. Johnson, M. R. Garasto, A. Battaglia-Mayer, L. Ercolani, L. Bianchi, F. Lacquaniti, and R. Caminiti (1997). "Combination of Hand and Gaze Signals During Reaching: Activity in Parietal Area $7 \mathrm{~m}$ of the Monkey". Journal of Neurophysiology 77.2, pp. 1034-1038. DOI: 10.1152/jn.1997.77.2.1034.

Ferraina, S., E. Brunamonti, M. A. Giusti, S. Costa, A. Genovesio, and R. Caminiti (2009). "Reaching in Depth: Hand Position Dominates over Binocular Eye Position in the Rostral Superior Parietal Lobule". Journal of Neuroscience 29.37, pp. 1146111470. DOI: 10.1523/JNEUROSCI . 1305-09.2009.

Ferro, J. M. (1984). "Transient Inaccuracy in Reaching Caused by a Posterior Parietal Lobe Lesion." Journal of Neurology, Neurosurgery, and Psychiatry 47.9, pp. 10161019.

Fiehler, K., M. M. Bannert, M. Bischoff, C. Blecker, R. Stark, D. Vaitl, V. H. Franz, and F. Rösler (2011). "Working Memory Maintenance of Grasp-Target Information in the Human Posterior Parietal Cortex". Neurolmage 54.3, pp. 2401-2411. DOI: $10.1016 / j$.neuroimage.2010.09.080

Filimon, F., J. Nelson, D. Hagler, and M. Sereno (2007). "Human Cortical Representations for Reaching: Mirror Neurons for Execution, Observation, and Imagery". Neurolmage 37.4, pp. 1315-1328. DOI: $10.1016 / \mathrm{j}$. neuroimage.2007.06.008.

Filimon, F., J. D. Nelson, R.-S. Huang, and M. I. Sereno (2009). "Multiple Parietal Reach Regions in Humans: Cortical Representations for Visual and Proprioceptive Feedback during On-Line Reaching". Journal of Neuroscience 29.9, pp. 2961-2971. DOI: $10.1523 /$ JNEUROSCI. 3211-08.2009. 
Filippini, M., R. Breveglieri, M. A. Akhras, A. Bosco, E. Chinellato, and P. Fattori (2017). "Decoding Information for Grasping from the Macaque Dorsomedial Visual Stream". Journal of Neuroscience 37.16, pp. 4311-4322. DOI: 10.1523/ JNEUROSCI . 3077-16.2017.

Floegel, M. and C. A. Kell (2017). "Functional Hemispheric Asymmetries during the Planning and Manual Control of Virtual Avatar Movements". PLOS ONE 12.9. DOI: 10.1371 /journal . pone.0185152.

Fluet, M.-C., M. A. Baumann, and H. Scherberger (2010). "Context-Specific Grasp Movement Representation in Macaque Ventral Premotor Cortex". The Journal of Neuroscience: The Official Journal of the Society for Neuroscience 30.45, pp. 1517515184. DOI: 10.1523/ JNEUROSCI.3343-10.2010.

Foltys, H., I. G. Meister, J. Weidemann, R. Sparing, A. Thron, K. Willmes, R. Töpper, M. Hallett, and B. Boroojerdi (2003). "Power Grip Disinhibits the Ipsilateral Sensorimotor Cortex: A TMS and fMRI Study". Neurolmage 19.2, pp. 332-340. DOI: $10.1016 /$ S1053-8119(03)00107-1.

Foxe, J. J., G. R. Wylie, A. Martinez, C. E. Schroeder, D. C. Javitt, D. Guilfoyle, W. Ritter, and M. M. Murray (2002). "Auditory-Somatosensory Multisensory Processing in Auditory Association Cortex: An fMRI Study". Journal of Neurophysiology 88.1, pp. 540-543. DOI: 10.1152/jn.2002.88.1.540.

François-Brosseau, F.-E., K. Martinu, A. P. Strafella, M. Petrides, F. Simard, and O. Monchi (2009). "Basal Ganglia and Frontal Involvement in Self-Generated and Externally-Triggered Finger Movements in the Dominant and Non-Dominant Hand". The European Journal of Neuroscience 29.6, pp. 1277-1286. DOI: 10 . 1111/j.1460-9568.2009.06671.x.

Frey, S. H., D. Vinton, R. Norlund, and S. T. Grafton (2005). "Cortical Topography of Human Anterior Intraparietal Cortex Active during Visually Guided Grasping". Cognitive Brain Research 23.2, pp. 397-405. DOI: 10.1016/ j.cogbrainres . 2004.11.010.

Friston, K. J., L. Harrison, and W. Penny (2003). "Dynamic Causal Modelling". Neurolmage 19.4, pp. 1273-1302. DOI: 10.1016/s1053-8119(03)00202-7.

Friston, K. J., A. P. Holmes, K. J. Worsley, J.-P. Poline, C. D. Frith, and R. S. J. Frackowiak (1994a). "Statistical Parametric Maps in Functional Imaging: A General Linear Approach". Human Brain Mapping 2.4, pp. 189-210. DOI: 10.1002/hbm. 460020402 .

Friston, K. J., P. Jezzard, and R. Turner (1994b). "Analysis of Functional MRI TimeSeries". Human Brain Mapping 1.2, pp. 153-171. DOI: 10.1002/hbm.460010207. 
Friston, K. J., K. J. Worsley, R. S. J. Frackowiak, J. C. Mazziotta, and A. C. Evans (1994c). "Assessing the Significance of Focal Activations Using Their Spatial Extent". Human Brain Mapping 1.3, pp. 210-220. DOI: 10.1002/hbm.460010306.

Friston, K. J., J. T. Ashburner, S. Kiebel, T. Nichols, and W. D. Penny (2007). Statistical Parametric Mapping: The Analysis of Functional Brain Images. First. Amsterdam ; Boston: Elsevier Ltd, Oxford.

Gail, A., C. Klaes, and S. Westendorff (2009). "Implementation of Spatial Transformation Rules for Goal-Directed Reaching via Gain Modulation in Monkey Parietal and Premotor Cortex". Journal of Neuroscience 29.30, pp. 9490-9499. DOI: 10.1523/JNEUROSCI.1095-09.2009.

Gallese, V., L. Fadiga, L. Fogassi, and G. Rizzolatti (1996). "Action Recognition in the Premotor Cortex". Brain 119.2, pp. 593-609. DOI: 10.1093/brain/119.2.593.

Gallese, V., A. Murata, M. Kaseda, N. Niki, and H. Sakata (1994). "Deficit of Hand Preshaping after Muscimol Injection in Monkey Parietal Cortex". NeuroReport 5.12, p. 1525.

Galletti, C., P. P. Battaglini, and P. Fattori (1993). "Parietal Neurons Encoding Spatial Locations in Craniotopic Coordinates". Experimental Brain Research 96.2, pp. 221-229. DOI: 10.1007/BF00227102.

Galletti, C., P. Fattori, D. F. Kutz, and P. P. Battaglini (1997). "Arm MovementRelated Neurons in the Visual Area V6A of the Macaque Superior Parietal Lobule". European Journal of Neuroscience 9.2, pp. 410-413. DOI: $10.1111 / \mathrm{j} .1460-$ 9568.1997.tb01410.x.

Galletti, C., P. Fattori, D. F. Kutz, and M. Gamberini (1999). "Brain Location and Visual Topography of Cortical Area V6A in the Macaque Monkey". European Journal of Neuroscience 11.2, pp. 575-582. DOI: 10.1046/j.1460-9568.1999.00467 .x. Galletti, C., M. Gamberini, D. F. Kutz, I. Baldinotti, and P. Fattori (2005). "The Relationship between V6 and PO in Macaque Extrastriate Cortex". European Journal of Neuroscience 21.4, pp. 959-970. DOI: 10.1111/j.1460-9568.2005.03911 . x.

Galletti, C., D. F. Kutz, M. Gamberini, R. Breveglieri, and P. Fattori (2003). "Role of the Medial Parieto-Occipital Cortex in the Control of Reaching and Grasping Movements". Experimental Brain Research 153.2, pp. 158-170. DOI: 10.1007/ s00221-003-1589-z.

Gallivan, J. P., C. Cavina-Pratesi, and J. C. Culham (2009). "Is That within Reach? fMRI Reveals That the Human Superior Parieto-Occipital Cortex Encodes Objects Reachable by the Hand". Journal of Neuroscience 29.14, pp. 4381-4391. DOI: 10.1523/JNEUROSCI.0377-09.2009. 
Gallivan, J. P., C. S. Chapman, D. A. McLean, J. R. Flanagan, and J. C. Culham (2013a). "Activity Patterns in the Category-Selective Occipitotemporal Cortex Predict Upcoming Motor Actions". European Journal of Neuroscience 38.3, pp. 24082424. DOI: $10.1111 /$ ejn.12215.

Gallivan, J. P., D. A. McLean, K. F. Valyear, and J. C. Culham (2013b). “Decoding the Neural Mechanisms of Human Tool Use". eLife 2. Ed. by D. Angelaki, e00425. DOI: $10.7554 /$ eLife.00425.

Gamberini, M., C. Galletti, A. Bosco, R. Breveglieri, and P. Fattori (2011). "Is the Medial Posterior Parietal Area V6A a Single Functional Area?" Journal of Neuroscience 31.13, pp. 5145-5157. DOI: 10.1523/JNEUROSCI .5489-10.2011.

Gamberini, M., L. Passarelli, P. Fattori, and C. Galletti (2020). "Structural Connectivity and Functional Properties of the Macaque Superior Parietal Lobule". Brain Structure and Function 225.4, pp. 1349-1367. DOI: 10 . 1007/s00429-01901976-9.

Gao, Q., X. Duan, and H. Chen (2011). "Evaluation of Effective Connectivity of Motor Areas during Motor Imagery and Execution Using Conditional Granger Causality". Neurolmage 54.2, pp. 1280-1288. DOI: 10.1016/j.neuroimage.2010.08.071. Gardner, T., A. Aglinskas, and E. S. Cross (2017). "Using Guitar Learning to Probe the Action Observation Network's Response to Visuomotor Familiarity". Neurolmage 156, pp. 174-189. DOI: 10.1016/j.neuroimage.2017.04.060.

Gentile, G., V. I. Petkova, and H. H. Ehrsson (2011). "Integration of Visual and Tactile Signals From the Hand in the Human Brain: An fMRI Study". Journal of Neurophysiology 105.2, pp. 910-922. DOI: 10.1152/jn.00840.2010.

Gentilucci, M., S. Chieffi, M. Scarpa, and U. Castiello (1992). "Temporal Coupling between Transport and Grasp Components during Prehension Movements: Effects of Visual Perturbation". Behavioural Brain Research 47.1, pp. 71-82. DOI: 10 . 1016/S0166-4328(05)80253-0.

Gerardin, E., A. Sirigu, S. Lehéricy, J.-B. Poline, B. Gaymard, C. Marsault, Y. Agid, and D. Le Bihan (2000). "Partially Overlapping Neural Networks for Real and Imagined Hand Movements". Cerebral Cortex 10.11, pp. 1093-1104. DOI: 10 . 1093/cercor/10.11.1093.

Ghosh, S. and R. Gattera (1995). "A Comparison of the Ipsilateral Cortical Projections to the Dorsal and Ventral Subdivisions of the Macaque Premotor Cortex". Somatosensory \& Motor Research 12.3-4, pp. 359-378. DOI: 10 . 3109/ 08990229509093668 . 
Gibbon, J., R. M. Church, and W. H. Meck (1984). "Scalar Timing in Memory". Annals of the New York Academy of Sciences 423.1, pp. 52-77. DOI: 10.1111/ j.1749-6632.1984.tb23417.x.

Giesbrecht, B., M. G. Woldorff, A. W. Song, and G. R. Mangun (2003). "Neural Mechanisms of Top-down Control during Spatial and Feature Attention". Neurolmage, p. 17.

Giorgio, J., V. M. Karlaftis, R. Wang, Y. Shen, P. Tino, A. Welchman, and Z. Kourtzi (2018). "Functional Brain Networks for Learning Predictive Statistics". Cortex; a Journal Devoted to the Study of the Nervous System and Behavior 107, pp. 204219. DOI: $10.1016 / \mathrm{j}$. cortex.2017.08.014.

Glover, S. (2003). "Optic Ataxia as a Deficit Specific to the On-Line Control of Actions". Neuroscience \& Biobehavioral Reviews 27.5, pp. 447-456. DOI: 10. 1016/S0149-7634(03)00072-1.

Goodale, M. A. (2011). "Transforming Vision into Action". Vision Research. Vision Research 50th Anniversary Issue: Part 2 51.13, pp. 1567-1587. DOI: 10.1016/j . visres.2010.07.027.

Görgen, K., M. N. Hebart, C. Allefeld, and J.-D. Haynes (2017). "The Same Analysis Approach: Practical Protection against the Pitfalls of Novel Neuroimaging Analysis Methods". Neurolmage. DOI: $10.1016 /$ j.neuroimage.2017.12.083.

Grabner, G., A. L. Janke, M. M. Budge, D. Smith, J. Pruessner, and D. L. Collins (2006). "Symmetric Atlasing and Model Based Segmentation: An Application to the Hippocampus in Older Adults". Medical Image Computing and ComputerAssisted Intervention - MICCAI 2006. Ed. by R. Larsen, M. Nielsen, and J. Sporring. Lecture Notes in Computer Science. Berlin, Heidelberg: Springer, pp. 58-66. DOI: 10.1007/11866763_8.

Grafton, S. T., M. A. Arbib, L. Fadiga, and G. Rizzolatti (1996). "Localization of Grasp Representations in Humans by Positron Emission Tomography". Experimental Brain Research 112.1, pp. 103-111. DOI: 10.1007/BF00227183.

Granger, C. W. J. (1969). "Investigating Causal Relations by Econometric Models and Cross-Spectral Methods". Econometrica 37.3, pp. 424-438. DOI: $10.2307 /$ 1912791 .

Grefkes, C., S. B. Eickhoff, D. A. Nowak, M. Dafotakis, and G. R. Fink (2008). "Dynamic Intra- and Interhemispheric Interactions during Unilateral and Bilateral Hand Movements Assessed with fMRI and DCM". Neurolmage 41.4, pp. 13821394. DOI: $10.1016 / \mathrm{j}$.neuroimage.2008.03.048. 
Grefkes, C. and G. R. Fink (2005). "REVIEW: The Functional Organization of the Intraparietal Sulcus in Humans and Monkeys". Journal of Anatomy 207.1, pp. 317. DOI: $10.1111 / \mathrm{j} .1469-7580.2005 .00426 . \mathrm{x}$.

Grefkes, C., P. H. Weiss, K. Zilles, and G. R. Fink (2002). "Crossmodal Processing of Object Features in Human Anterior Intraparietal Cortex: An fMRI Study Implies Equivalencies between Humans and Monkeys". Neuron 35.1, pp. 173-184. DOI: 10.1016/S0896-6273(02)00741-9.

Greulich, R. S., R. Adam, S. Everling, and H. Scherberger (2020). "Shared Functional Connectivity between the Dorso-Medial and Dorso-Ventral Streams in Macaques". Scientific Reports 10.1, p. 18610. DOI: 10.1038/s41598-020-75219-x.

Grèzes, J., M. Tucker, J. Armony, R. Ellis, and R. E. Passingham (2003). "Objects Automatically Potentiate Action: An fMRI Study of Implicit Processing". European Journal of Neuroscience 17.12, pp. 2735-2740. DOI: 10.1046/j.1460-9568. 2003.02695.x.

Grol, M. J., J. Majdandzić, K. E. Stephan, L. Verhagen, H. C. Dijkerman, H. Bekkering, F. A. J. Verstraten, and I. Toni (2007). "Parieto-Frontal Connectivity during Visually Guided Grasping". The Journal of Neuroscience: The Official Journal of the Society for Neuroscience 27.44, pp. 11877-11887. DOI: 10.1523/JNEUROSCI. 3923-07.2007.

Grondin, S. (2010). "Timing and Time Perception: A Review of Recent Behavioral and Neuroscience Findings and Theoretical Directions". Attention, Perception, \& Psychophysics 72.3, pp. 561-582. DOI: 10.3758/APP.72.3.561.

Gruber, O., A. Kleinschmidt, F. Binkofski, H. Steinmetz, and D. Y. von Cramon (2000). "Cerebral Correlates of Working Memory for Temporal Information". NeuroReport 11.8, pp. 1689-1693.

Grützmann, R., T. Endrass, C. Kaufmann, E. Allen, T. Eichele, and N. Kathmann (2016). "Presupplementary Motor Area Contributes to Altered Error Monitoring in Obsessive-Compulsive Disorder". Biological Psychiatry 80.7, pp. 562-571. DOI: 10.1016/j.biopsych.2014.12.010.

Haaxma, R. and H. G. J. M. Kuypers (1975). "Intrahemispheric Cortical Connexions and Visual Guidance of Hand and Finger Movements in the Rhusus Monkey". Brain 98.2, pp. 239-260. DOI: 10.1093/brain/98.2.239

Hadjidimitrakis, K., F. Bertozzi, R. Breveglieri, P. Fattori, and C. Galletti (2014). "Body-Centered, Mixed, but Not Hand-Centered Coding of Visual Targets in the Medial Posterior Parietal Cortex During Reaches in 3D Space". Cerebral Cortex 24.12, pp. 3209-3220. DOI: $10.1093 /$ cercor/bht181. 
Hadjidimitrakis, K., G. Dal Bo', R. Breveglieri, C. Galletti, and P. Fattori (2015). "Overlapping Representations for Reach Depth and Direction in Caudal Superior Parietal Lobule of Macaques". Journal of Neurophysiology 114.4, pp. 2340-2352. DOI: $10.1152 /$ jn.00486.2015.

Hadjidimitrakis, K., M. Ghodrati, R. Breveglieri, M. G. P. Rosa, and P. Fattori (2020). "Neural Coding of Action in Three Dimensions: Task- and Time-Invariant Reference Frames for Visuospatial and Motor-Related Activity in Parietal Area V6A". Journal of Comparative Neurology 528.17, pp. 3108-3122. DOI: 10.1002/cne.24889.

Haggard, P. (2008). "Human Volition: Towards a Neuroscience of Will". Nature Reviews Neuroscience 9.12, pp. 934-946. DOI: 10.1038/nrn2497.

Haith, A. M., J. Pakpoor, and J. W. Krakauer (2016). "Independence of Movement Preparation and Movement Initiation". The Journal of Neuroscience 36.10, pp. 3007-3015. DOI: 10.1523/JNEUROSCI . 3245-15.2016.

Hanakawa, T., M. A. Dimyan, and M. Hallett (2008). "Motor Planning, Imagery, and Execution in the Distributed Motor Network: A Time-Course Study with Functional MRI". Cerebral Cortex (New York, NY) 18.12, pp. 2775-2788. DOI: 10.1093/ cercor/bhn036.

Hanson, S. J., T. Matsuka, and J. V. Haxby (2004). "Combinatorial Codes in Ventral Temporal Lobe for Object Recognition: Haxby (2001) Revisited: Is There a "Face" Area?" Neurolmage 23.1, pp. 156-166. DOI: 10.1016/j . neuroimage.2004.05. 020

Harrington, D. L. and M. Jahanshahi (2016). "Reconfiguration of Striatal Connectivity for Timing and Action". Current Opinion in Behavioral Sciences. Time in Perception and Action 8, pp. 78-84. DOI: 10.1016/j.cobeha.2016.02.007.

Haslinger, B., P. Erhard, E. Altenmüller, A. Hennenlotter, M. Schwaiger, H. Gräfin von Einsiedel, E. Rummeny, B. Conrad, and A. O. Ceballos-Baumann (2004). "Reduced Recruitment of Motor Association Areas during Bimanual Coordination in Concert Pianists". Human Brain Mapping 22.3, pp. 206-215. DOI: 10.1002/ hbm. 20028 .

Hauber, W. (1998). "Involvement of Basal Ganglia Transmitter Systems in Movement Initiation". Progress in Neurobiology 56.5, pp. 507-540. DOI: 10.1016/S03010082(98)00041-0.

Hausfeld, L., G. Valente, and E. Formisano (2014). "Multiclass fMRI Data Decoding and Visualization Using Supervised Self-Organizing Maps". Neurolmage 96, pp. 5466. DOI: $10.1016 / \mathrm{j}$.neuroimage.2014.02.006. 
Haxby, J. V. (2012). "Multivariate Pattern Analysis of fMRI: The Early Beginnings". Neurolmage. 20 YEARS OF fMRI 62.2, pp. 852-855. DOI: $10.1016 /$ j.neuroimage. 2012.03.016.

Haxby, J. V., M. I. Gobbini, M. L. Furey, A. Ishai, J. L. Schouten, and P. Pietrini (2001). "Distributed and Overlapping Representations of Faces and Objects in Ventral Temporal Cortex". Science 293.5539, pp. 2425-2430. DOI: $10.1126 /$ science.1063736.

Haynes, J.-D. and G. Rees (2006). "Decoding Mental States from Brain Activity in Humans". Nature Reviews Neuroscience 7.7, pp. 523-534. DOI: $10.1038 /$ nrn1931.

He, S., R. Dum, and P. Strick (1993). "Topographic Organization of Corticospinal Projections from the Frontal Lobe: Motor Areas on the Lateral Surface of the Hemisphere". The Journal of neuroscience : the official journal of the Society for Neuroscience 13, pp. 952-80. DOI: 10.1523/JNEUROSCI.13-03-00952.1993.

Heitger, M. H., M. J.-M. Macé, J. Jastorff, S. P. Swinnen, and G. A. Orban (2012).

"Cortical Regions Involved in the Observation of Bimanual Actions". Journal of Neurophysiology 108.9, pp. 2594-2611. DOI: 10.1152/jn.00408.2012.

Heyes, C. (2010). "Where Do Mirror Neurons Come From?" Neuroscience \& Biobehavioral Reviews 34.4, pp. 575-583. DOI: 10.1016/j .neubiorev.2009.11.007. Higuchi, S., H. Holle, N. Roberts, S. B. Eickhoff, and S. Vogt (2012). "Imitation and Observational Learning of Hand Actions: Prefrontal Involvement and Connectivity". Neurolmage 59.2, pp. 1668-1683. DOI: 10.1016/j.neuroimage.2011.09.021. Hinkley, L. B. N., L. A. Krubitzer, J. Padberg, and E. A. Disbrow (2009). "VisualManual Exploration and Posterior Parietal Cortex in Humans". Journal of Neurophysiology 102.6, pp. 3433-3446. DOI: 10.1152/jn.90785.2008.

Hinton, S. C., D. L. Harrington, J. R. Binder, S. Durgerian, and S. M. Rao (2004). "Neural Systems Supporting Timing and Chronometric Counting: An FMRI Study". Cognitive Brain Research 21.2, pp. 183-192. DOI: 10.1016/ j.cogbrainres. 2004.04.009.

Hirose, S. (2020). "Valid and Powerful Group Statistics for Decoding Accuracy: Information Prevalence Inference Using the i-Th Order Statistic (i-Test)". bioRxiv, p. 578930. DOI: 10.1101/578930.

Horovitz, S. G., C. Gallea, M. A. Najee-ullah, and M. Hallett (2013). "Functional Anatomy of Writing with the Dominant Hand". PLOS ONE 8.7. Ed. by N. M. Maurits, e67931. DOI: 10.1371/journal.pone.0067931. 
Hoshi, E. and J. Tanji (2002). "Contrasting Neuronal Activity in the Dorsal and Ventral Premotor Areas During Preparation to Reach". Journal of Neurophysiology 87.2, pp. 1123-1128. DOI: 10.1152/jn.00496.2001.

Hove, M. J., M. T. Fairhurst, S. A. Kotz, and P. E. Keller (2013). "Synchronizing with Auditory and Visual Rhythms: An fMRI Assessment of Modality Differences and Modality Appropriateness". Neurolmage 67, pp. 313-321. DOI: $10.1016 / \mathrm{j}$. neuroimage.2012.11.032.

Huettel, S. A., A. W. Song, and G. McCarthy (2014). Functional Magnetic Resonance Imaging. 3rd ed. 2014 Edition. Sunderland, Massachusetts, U.S.A: Sinauer.

Hultin, L., P. Rossini, G. L. Romani, P. Högstedt, F. Tecchio, and V. Pizzella (1996).

"Neuromagnetic Localization of the Late Component of the Contingent Negative Variation". Electroencephalography and Clinical Neurophysiology 98.6, pp. 435448. DOI: $10.1016 / 0013-4694$ (96)95507-8.

Hummel, F., R. Saur, S. Lasogga, C. Plewnia, M. Erb, D. Wildgruber, W. Grodd, and C. Gerloff (2004). "To Act or Not to Act. Neural Correlates of Executive Control of Learned Motor Behavior", p. 11.

Hwang, E. J., M. Hauschild, M. Wilke, and R. A. Andersen (2012). "Inactivation of the Parietal Reach Region Causes Optic Ataxia, Impairing Reaches but Not Saccades". Neuron 76.5, pp. 1021-1029. DOI: 10.1016/j. neuron.2012.10.030. Hyvärinen, J. and A. Poranen (1974). "Function of the Parietal Associative Area 7 as Revealed from Cellular Discharges in Alert Monkeys". Brain 97.4, pp. 673-692. DOI: $10.1093 /$ brain/97.4.673.

lannaccone, R., T. U. Hauser, P. Staempfli, S. Walitza, D. Brandeis, and S. Brem (2015). "Conflict Monitoring and Error Processing: New Insights from Simultaneous EEG-fMRI". Neurolmage 105, pp. 395-407. DOI: 10.1016/j.neuroimage. 2014.10.028.

Intveld, R. W., B. Dann, J. A. Michaels, and H. Scherberger (2018). "Neural Coding of Intended and Executed Grasp Force in Macaque Areas AIP, F5, and M1". Scientific Reports 8.1, pp. 1-16. DOI: 10.1038/s41598-018-35488-z.

Iwaniuk, A. N. and I. Q. Whishaw (2000). "On the Origin of Skilled Forelimb Movements". Trends in Neurosciences 23.8, pp. 372-376. DOI: 10.1016 / S01662236(00) 01618-0.

Jackson, C. P. T., R. Bowtell, P. G. Morris, and S. R. Jackson (2008). “A Novel MRCompatible Device for Providing Forces to the Human Finger during Functional Neuroimaging Studies". Neurolmage 40.4, pp. 1731-1737. DOI: $10.1016 / \mathrm{j}$. neuroimage.2008.02.001. 
Jamadar, S., M. Hughes, W. Fulham, P. Michie, and F. Karayanidis (2010). "The Spatial and Temporal Dynamics of Anticipatory Preparation and Response Inhibition in Task-Switching". Neurolmage 51.1, pp. 432-449. DOI: $10.1016 / \mathrm{j}$. neuroimage.2010.01.090

Jamison, H. L., K. E. Watkins, D. V. M. Bishop, and P. M. Matthews (2006).

"Hemispheric Specialization for Processing Auditory Nonspeech Stimuli". Cerebral Cortex 16.9, pp. 1266-1275. DOI: 10.1093/cercor/bhj068.

Jäncke, L., R. Loose, K. Lutz, K. Specht, and N. J. Shah (2000). "Cortical Activations during Paced Finger-Tapping Applying Visual and Auditory Pacing Stimuli". Cognitive Brain Research 10.1, pp. 51-66. DOI: 10.1016/S0926-6410(00)00022-7. Jankowski, J., L. Scheef, C. Huppe, and H. Boecker (2009). "Distinct Striatal Regions for Planning and Executing Novel and Automated Movement Sequences". Neurolmage 44.4, pp. 1369-1379. DOI: 10.1016/j .neuroimage.2008.10.059. Jastorff, J., R. O. Abdollahi, F. Fasano, and G. A. Orban (2015). "Seeing Biological Actions in 3D: An fMRI Study". Human Brain Mapping 37.1, pp. 203-219. DOI: 10.1002/hbm. 23020 .

Jeannerod, M. (1981). "Intersegmental Coordination during Reaching at Natural Visual Objects". Attention and performance IX, pp. 153-168.

- (1984). "The Timing of Natural Prehension Movements". Journal of Motor Behavior 16.3, pp. 235-254. DOI: 10.1080/00222895.1984.10735319.

- (1986). "The Formation of Finger Grip during Prehension. A Cortically Mediated Visuomotor Pattern". Behavioural Brain Research 19.2, pp. 99-116. DOI: 10 . 1016/0166-4328(86)90008-2.

- (1999). "Visuomotor Channels: Their Integration in Goal-Directed Prehension". Human Movement Science 18.2, pp. 201-218. DOI: 10.1016/S0167-9457(99) 00008-1.

Jeannerod, M., M. A. Arbib, G. Rizzolatti, and H. Sakata (1995). "Grasping Objects:

The Cortical Mechanisms of Visuomotor Transformation". Trends in Neurosciences 18.7, pp. 314-320. DOI: $10.1016 / 0166-2236$ (95)93921-J.

Jenkins, I. H., M. Jahanshahi, M. Jueptner, R. E. Passingham, and D. J. Brooks (2000). "Self-Initiated versus Externally Triggered Movements II. The Effect of Movement Predictability on Regional Cerebral Blood Flow". Brain 123.6, pp. 12161228. DOI: 10.1093/brain/123.6.1216.

Jerjian, S. J., M. Sahani, and A. Kraskov (2020). "Movement Initiation and Grasp Representation in Premotor and Primary Motor Cortex Mirror Neurons". eLife 9. Jia, X.-Z., J. Wang, H.-Y. Sun, H. Zhang, W. Liao, Z. Wang, C.-G. Yan, X.-W. Song, and Y.-F. Zang (2019). "RESTplus: An Improved Toolkit for Resting-State 
Functional Magnetic Resonance Imaging Data Processing". Science Bulletin 64.14, pp. 953-954. DOI: 10.1016/j.scib.2019.05.008.

Jimura, K. and R. A. Poldrack (2012). "Analyses of Regional-Average Activation and Multivoxel Pattern Information Tell Complementary Stories". Neuropsychologia. Multivoxel Pattern Analysis and Cognitive Theories 50.4, pp. 544-552. DOI: 10 . 1016/j.neuropsychologia.2011.11.007

Johnson, P. B., S. Ferraina, L. Bianchi, and R. Caminiti (1996). "Cortical Networks for Visual Reaching: Physiological and Anatomical Organization of Frontal and Parietal Lobe Arm Regions". Cerebral Cortex (New York, N.Y.: 1991) 6.2, pp. 102119. DOI: $10.1093 /$ cercor/6.2.102.

Johnson, P. B., S. Ferraina, and R. Caminiti (1993). "Cortical Networks for Visual Reaching". Experimental Brain Research 97.2, pp. 361-365.

Kakei, S., D. S. Hoffman, and P. L. Strick (2001). "Direction of Action Is Represented in the Ventral Premotor Cortex". Nature Neuroscience 4.10, p. 1020. DOI: 10 . $1038 / \mathrm{nn} 726$.

Kalaska, J. F., R. Caminiti, and A. P. Georgopoulos (1983). "Cortical Mechanisms Related to the Direction of Two-Dimensional Arm Movements: Relations in Parietal Area 5 and Comparison with Motor Cortex". Experimental Brain Research 51.2, pp. 247-260. DOI: 10.1007/BF00237200

Kalaska, J. F., P. Cisek, and N. Gosselin-Kessiby (2003). "Mechanisms of Selection and Guidance of Reaching Movements in the Parietal Lobe". Advances in Neurology 93, pp. 97-119.

Kamitani, Y. and F. Tong (2005). "Decoding the Visual and Subjective Contents of the Human Brain". Nature Neuroscience 8.5, pp. 679-685. DOI: 10.1038/nn1444. Kansaku, K., T. Hanakawa, T. Wu, and M. Hallett (2004). "A Shared Neural Network for Simple Reaction Time". Neurolmage 22.2, pp. 904-911. DOI: $10.1016 / \mathrm{j}$. neuroimage.2004.02.006.

Kansaku, K., A. Johnson, M.-L. Grillon, G. Garraux, N. Sadato, and M. Hallett (2006). "Neural Correlates of Counting of Sequential Sensory and Motor Events in the Human Brain". Neurolmage 31.2, pp. 649-660. DOI: 10.1016/j . neuroimage. 2005.12.023.

Karabanov, A., Ö. Blom, L. Forsman, and F. Ullén (2009). "The Dorsal Auditory Pathway Is Involved in Performance of Both Visual and Auditory Rhythms". Neurolmage 44.2, pp. 480-488. DOI: $10.1016 / \mathrm{j}$. neuroimage.2008.08.047.

Karl, J. M. and I. Q. Whishaw (2013). "Different Evolutionary Origins for the Reach and the Grasp: An Explanation for Dual Visuomotor Channels in Primate Parietofrontal Cortex". Frontiers in Neurology 4. DOI: 10.3389/fneur.2013.00208. 
Karnath, H.-O. and M.-T. Perenin (2005). "Cortical Control of Visually Guided Reaching: Evidence from Patients with Optic Ataxia". Cerebral Cortex 15.10, pp. 1561-1569. DOI: 10.1093/cercor/bhi034.

Karpati, F. J., C. Giacosa, N. E. Foster, V. B. Penhune, and K. L. Hyde (2017). "Dance and Music Share Gray Matter Structural Correlates". Brain Research 1657, pp. 62-73. DOI: 10.1016/j.brainres.2016.11.029.

Kleber, B., R. Veit, N. Birbaumer, J. Gruzelier, and M. Lotze (2010). "The Brain of Opera Singers: Experience-Dependent Changes in Functional Activation". Cerebral Cortex 20.5, pp. 1144-1152. DOI: 10.1093/cercor/bhp177.

Klein, T. A., T. Endrass, N. Kathmann, J. Neumann, D. Y. von Cramon, and M. Ullsperger (2007). "Neural Correlates of Error Awareness". Neurolmage 34.4, pp. 1774-1781. DOI: $10.1016 / \mathrm{j}$.neuroimage.2006.11.014

Klöppel, S., T. van Eimeren, V. Glauche, A. Vongerichten, A. Münchau, R. S. Frackowiak, C. Büchel, C. Weiller, and H. R. Siebner (2007). "The Effect of Handedness on Cortical Motor Activation during Simple Bilateral Movements". Neurolmage 34.1, pp. 274-280. DOI: 10.1016/j.neuroimage.2006.08.038.

Koch, G., M. F. D. Olmo, B. Cheeran, S. Schippling, C. Caltagirone, J. Driver, and J. C. Rothwell (2008). "Functional Interplay between Posterior Parietal and Ipsilateral Motor Cortex Revealed by Twin-Coil Transcranial Magnetic Stimulation during Reach Planning toward Contralateral Space". Journal of Neuroscience 28.23, pp. 5944-5953. DOI: 10.1523/JNEUROSCI.0957-08.2008.

Koch, M. A., D. G. Norris, and M. Hund-Georgiadis (2002). "An Investigation of Functional and Anatomical Connectivity Using Magnetic Resonance Imaging". Neurolmage 16.1, pp. 241-250. DOI: 10.1006/nimg.2001.1052.

Koeneke, S., K. Lutz, T. Wüstenberg, and L. Jäncke (2004). "Bimanual versus Unimanual Coordination: What Makes the Difference?" Neurolmage 22.3, pp. 13361350. DOI: $10.1016 / \mathrm{j}$.neuroimage.2004.03.012.

Konoike, N., Y. Kotozaki, S. Miyachi, C. M. Miyauchi, Y. Yomogida, Y. Akimoto, K. Kuraoka, M. Sugiura, R. Kawashima, and K. Nakamura (2012). "Rhythm Information Represented in the Fronto-Parieto-Cerebellar Motor System". Neurolmage 63.1, pp. 328-338. DOI: 10.1016/j.neuroimage.2012.07.002.

Kornhuber, H. H. and L. Deecke (1965). "Hirnpotentialänderungen bei Willkürbewegungen und passiven Bewegungen des Menschen: Bereitschaftspotential und reafferente Potentiale". Pflüger's Archiv für die gesamte Physiologie des Menschen und der Tiere 284.1, pp. 1-17. DOI: 10.1007/BF00412364. 
Kornysheva, K. and R. I. Schubotz (2011). "Impairment of Auditory-Motor Timing and Compensatory Reorganization after Ventral Premotor Cortex Stimulation". PLOS ONE 6.6. DOI: $10.1371 /$ journal . pone.0021421.

Koshland, G. F., J. C. Galloway, and C. J. Nevoret-Bell (2000). "Control of the Wrist in Three-Joint Arm Movements to Multiple Directions in the Horizontal Plane". Journal of Neurophysiology 83.5, pp. 3188-3195. DOI: $10.1152 /$ jn.2000.83. 5.3188

Koski, L., A. Wohlschläger, H. Bekkering, R. P. Woods, M.-C. Dubeau, J. C. Mazziotta, and M. lacoboni (2002). "Modulation of Motor and Premotor Activity during Imitation of Target-Directed Actions". Cerebral Cortex 12.8, pp. 847-855. DOI: 10.1093/cercor/12.8.847

Kriegeskorte, N., R. Goebel, and P. Bandettini (2006). "Information-Based Functional Brain Mapping". Proceedings of the National Academy of Sciences of the United States of America 103.10, pp. 3863-3868. DOI: 10.1073/pnas.0600244103.

Kroeger, J., T. Bäumer, M. Jonas, J. C. Rothwell, H. R. Siebner, and A. Münchau (2010). "Charting the Excitability of Premotor to Motor Connections While Withholding or Initiating a Selected Movement". European Journal of Neuroscience 32.10, pp. 1771-1779. DOI: 10.1111/j.1460-9568.2010.07442.x.

Króliczak, G., C. Cavina-Pratesi, D. A. Goodman, and J. C. Culham (2007). "What Does the Brain Do When You Fake It? An fMRI Study of Pantomimed and Real Grasping". Journal of Neurophysiology 97.3, pp. 2410-2422. DOI: $10.1152 /$ jn. 00778.2006 .

Kubota, K. and I. Hamada (1978). "Visual Tracking and Neuron Activity in the Post-Arcuate Area in Monkeys". Journal De Physiologie 74.3, pp. 297-312.

Kuhtz-Buschbeck, J. P., R. Gilster, S. Wolff, S. Ulmer, H. Siebner, and O. Jansen (2008). "Brain Activity Is Similar during Precision and Power Gripping with Light Force: An fMRI Study". Neurolmage 40.4, pp. 1469-1481. DOI: $10.1016 / \mathrm{j}$. neuroimage.2008.01.037.

Kung, S.-J., J. L. Chen, R. J. Zatorre, and V. B. Penhune (2012). "Interacting Cortical and Basal Ganglia Networks Underlying Finding and Tapping to the Musical Beat". Journal of Cognitive Neuroscience 25.3, pp. 401-420. DOI: 10.1162 / jocn_a_ 00325.

Kurata, K. and J. Tanji (1986). "Premotor Cortex Neurons in Macaques: Activity before Distal and Proximal Forelimb Movements". Journal of Neuroscience 6.2, pp. 403-411. DOI: 10.1523/JNEUROSCI.06-02-00403.1986.

Kurata, K. and S. P. Wise (1988). "Premotor and Supplementary Motor Cortex in Rhesus Monkeys: Neuronal Activity during Externally- and Internally-Instructed 
Motor Tasks". Experimental Brain Research 72.2, pp. 237-248. DOI: $10.1007 /$ BF00250247.

Kurata, K. (1991). "Corticocortical Inputs to the Dorsal and Ventral Aspects of the

Premotor Cortex of Macaque Monkeys". Neuroscience Research 12.1, pp. 263-280. DOI: 10.1016/0168-0102(91)90116-G.

Lacquaniti, F., E. Guigon, L. Bianchi, S. Ferraina, and R. Caminiti (1995). "Representing Spatial Information for Limb Movement: Role of Area 5 in the Monkey". Cerebral Cortex 5.5, pp. 391-409. DOI: 10.1093/cercor/5.5.391.

Langner, R., M. A. Sternkopf, T. S. Kellermann, C. Grefkes, F. Kurth, F. Schneider, K. Zilles, and S. B. Eickhoff (2013). "Translating Working Memory into Action: Behavioral and Neural Evidence for Using Motor Representations in Encoding Visuo-spatial Sequences". Human Brain Mapping 35.7, pp. 3465-3484. DOI: $10.1002 / \mathrm{hbm} .22415$

Lara, A. H., J. P. Cunningham, and M. M. Churchland (2018). "Different Population Dynamics in the Supplementary Motor Area and Motor Cortex during Reaching". Nature Communications 9.1, p. 2754. DOI: 10.1038/s41467-018-05146-z. Lau, H., R. Rogers, N. Ramnani, and R. Passingham (2004). "Willed Action and Attention to the Selection of Action". Neurolmage 21.4, pp. 1407-1415. DOI: 10.1016/j.neuroimage.2003.10.034

Laurens, K. R., K. A. Kiehl, and P. F. Liddle (2004). "A Supramodal Limbic-paralimbicneocortical Network Supports Goal-directed Stimulus Processing". Human Brain Mapping 24.1, pp. 35-49. DOI: 10.1002/hbm. 20062.

Lavrysen, A., E. Heremans, R. Peeters, N. Wenderoth, W. F. Helsen, P. Feys, and S. P. Swinnen (2008). "Hemispheric Asymmetries in Eye-Hand Coordination". Neurolmage 39.4, pp. 1938-1949. DOI: 10.1016/j . neuroimage. 2007.10.007.

Lehéricy, S., E. Bardinet, L. Tremblay, P.-F. Van de Moortele, J.-B. Pochon, D. Dormont, D.-S. Kim, J. Yelnik, and K. Ugurbil (2006). "Motor Control in Basal Ganglia Circuits Using fMRI and Brain Atlas Approaches". Cerebral Cortex 16.2, pp. 149-161. DOI: 10.1093/cercor/bhi089.

Lehmann, C., M. Herdener, F. Esposito, D. Hubl, F. di Salle, K. Scheffler, D. R. Bach, A. Federspiel, R. Kretz, T. Dierks, and E. Seifritz (2006). "Differential Patterns of Multisensory Interactions in Core and Belt Areas of Human Auditory Cortex". Neurolmage 31.1, pp. 294-300. DOI: 10.1016/j.neuroimage.2005.12.038.

Lehmann, S. J. and H. Scherberger (2013). "Reach and Gaze Representations in Macaque Parietal and Premotor Grasp Areas". Journal of Neuroscience 33.16, pp. 7038-7049. DOI: 10.1523/JNEUROSCI .5568-12.2013. 
Leonard, C. M., D. S. Glendinning, T. Wilfong, B. Y. Cooper, and C. J. Vierck (1992). "Alterations of Natural Hand Movements after Interruption of Fasciculus Cuneatus in the Macaque". Somatosensory \& Motor Research 9.1, pp. 75-89. DOI: 10.3109/08990229209144764.

Lerner, A., A. Bagic, T. Hanakawa, E. A. Boudreau, F. Pagan, Z. Mari, W. BaraJimenez, M. Aksu, S. Sato, D. L. Murphy, and M. Hallett (2009). "Involvement of Insula and Cingulate Cortices in Control and Suppression of Natural Urges". Cerebral Cortex (New York, NY) 19.1, pp. 218-223. DOI: 10.1093/cercor/ bhn074

Leung, H.-C. and W. Cai (2007). "Common and Differential Ventrolateral Prefrontal Activity during Inhibition of Hand and Eye Movements". The Journal of Neuroscience 27.37, pp. 9893-9900. DOI: 10.1523/JNEUROSCI . 2837-07.2007.

Levy, I., D. Schluppeck, D. J. Heeger, and P. W. Glimcher (2007). "Specificity of Human Cortical Areas for Reaches and Saccades". Journal of Neuroscience 27.17, pp. 4687-4696. DOI: 10.1523/JNEUROSCI . 0459-07.2007.

Lewis, J. W. and D. C. Van Essen (1994). "Connections of Area VIP with Area MIP and Other Architectonically Identified Areas of the Intraparietal Sulcus in Macaque Monkeys". Soc Neurosci Abstr. Vol. 20, p. 724.

Lewis, J. W. and D. C. V. Essen (2000). "Mapping of Architectonic Subdivisions in the Macaque Monkey, with Emphasis on Parieto-Occipital Cortex". Journal of Comparative Neurology 428.1, pp. 79-111. DOI: 10.1002/1096-9861(20001204)428: 1<79: :AID-CNE7>3.0.CO;2-Q.

Lewis, P. A., A. M. Wing, P. A. Pope, P. Praamstra, and R. C. Miall (2004). "Brain Activity Correlates Differentially with Increasing Temporal Complexity of Rhythms during Initialisation, Synchronisation, and Continuation Phases of Paced Finger Tapping". Neuropsychologia 42.10, pp. 1301-1312. DOI: $10.1016 / \mathrm{j}$. neuropsychologia.2004.03.001.

Lewis, P. A. and R. C. Miall (2002). "Brain Activity during Non-Automatic Motor Production of Discrete Multi-Second Intervals". NeuroReport 13.14, pp. 17311735.

Lewis, R. and U. Noppeney (2010). "Audiovisual Synchrony Improves Motion Discrimination via Enhanced Connectivity between Early Visual and Auditory Areas". The Journal of Neuroscience 30.37, pp. 12329-12339. DOI: 10.1523/JNEUROSCI. 5745-09.2010.

Li, J. and C. ladecola (1994). "Nitric Oxide and Adenosine Mediate Vasodilation during Functional Activation in Cerebellar Cortex". Neuropharmacology 33.11, pp. 1453-1461. DOI: 10.1016/0028-3908(94)90049-3. 
Libet, B., C. A. Gleason, E. W. Wright, and D. K. Pearl (1983). "Time of Conscious Intention to Act in Relation to Onset of Cerebral Activity (Readiness-Potential). The Unconscious Initiation of a Freely Voluntary Act". Brain 106.3, pp. 623-642. DOI: $10.1093 /$ brain/106.3.623.

Lissek, S., M. Hausmann, F. Knossalla, S. Peters, V. Nicolas, O. Güntürkün, and M. Tegenthoff (2007). "Sex Differences in Cortical and Subcortical Recruitment during Simple and Complex Motor Control: An fMRI Study". Neurolmage 37.3, pp. 912-926. DOI: 10.1016/j.neuroimage.2007.05.037.

Liu, M.-J., C.-H. Xiong, and D. Hu (2016). "Assessing the Manipulative Potentials of Monkeys, Apes and Humans from Hand Proportions: Implications for Hand Evolution". Proceedings of the Royal Society B: Biological Sciences 283.1843, p. 20161923. DOI: 10.1098/rspb.2016.1923.

Logothetis, N. K. (2008). "What We Can Do and What We Cannot Do with fMRI". Nature 453.7197, pp. 869-878. DOI: 10.1038/nature06976.

Logothetis, N. K. and B. A. Wandell (2004). "Interpreting the BOLD Signal". Annual Review of Physiology 66.1, pp. 735-769. DOI: 10.1146/annurev.physiol.66. 082602.092845 .

Lotze, M., M. Erb, H. Flor, E. Huelsmann, B. Godde, and W. Grodd (2000). "fMRI Evaluation of Somatotopic Representation in Human Primary Motor Cortex". Neurolmage 11.5, pp. 473-481. DOI: 10.1006/nimg.2000.0556.

Lotze, M., G. Scheler, H.-R. Tan, C. Braun, and N. Birbaumer (2003). "The Musician's Brain: Functional Imaging of Amateurs and Professionals during Performance and Imagery". Neurolmage 20.3, pp. 1817-1829. DOI: $10.1016 / \mathrm{j}$. neuroimage.2003.07.018.

Lowe, M. X., J. P. Gallivan, S. Ferber, and J. S. Cant (2016). "Feature Diagnosticity and Task Context Shape Activity in Human Scene-Selective Cortex". Neurolmage 125, pp. 681-692. DOI: 10.1016/j.neuroimage.2015.10.089.

Lu, M.-K., N. Arai, C.-H. Tsai, and U. Ziemann (2012). "Movement Related Cortical Potentials of Cued versus Self-Initiated Movements: Double Dissociated Modulation by Dorsal Premotor Cortex versus Supplementary Motor Area rTMS". Human Brain Mapping 33.4, pp. 824-839. DOI: 10.1002/hbm. 21248.

Luppino, G., A. Murata, P. Govoni, and M. Matelli (1999). "Largely Segregated Parietofrontal Connections Linking Rostral Intraparietal Cortex (Areas AIP and VIP) and the Ventral Premotor Cortex (Areas F5 and F4)". Experimental Brain Research 128.1, pp. 181-187. DOI: 10.1007/s002210050833. 
Macaluso, E., C. D. Frith, and J. Driver (2007). "Delay Activity and Sensory-Motor Translation During Planned Eye or Hand Movements to Visual or Tactile Targets". Journal of Neurophysiology 98.5, pp. 3081-3094. DOI: 10.1152/jn.00192.2007. MacNeilage, P. F. (1990). "Grasping in Modern Primates: The Evolutionary Context". Vision and action: the control of grasping. Norwood: Ablex, pp. 1-13.

Maguire, R., A. Broerse, B. de Jong, F. Cornelissen, L. Meiners, K. Leenders, and J. den Boer (2003). "Evidence of Enhancement of Spatial Attention during Inhibition of a Visuo-Motor Response". Neurolmage 20.2, pp. 1339-1345. DOI: $10.1016 /$ S1053-8119(03) 00402-6.

Mancini, L., O. Ciccarelli, F. Manfredonia, J. S. Thornton, F. Agosta, F. Barkhof, C. Beckmann, N. De Stefano, C. Enzinger, F. Fazekas, M. Filippi, A. Gass, J. G. Hirsch, H. Johansen-Berg, L. Kappos, T. Korteweg, S. C. Manson, S. Marino, P. M. Matthews, X. Montalban, J. Palace, C. Polman, M. Rocca, S. Ropele, A. Rovira, C. Wegner, K. Friston, A. Thompson, and T. Yousry (2009). "Short-Term Adaptation to a Simple Motor Task: A Physiological Process Preserved in Multiple Sclerosis". Neurolmage 45.2, pp. 500-511. DOI: $10.1016 / \mathrm{j}$. neuroimage.2008.12.006.

Manto, M., J. M. Bower, A. B. Conforto, J. M. Delgado-García, S. N. F. da Guarda, M. Gerwig, C. Habas, N. Hagura, R. B. Ivry, P. Mariën, M. Molinari, E. Naito, D. A. Nowak, N. O. Ben Taib, D. Pelisson, C. D. Tesche, C. Tilikete, and D. Timmann (2012). "Consensus Paper: Roles of the Cerebellum in Motor Control-The Diversity of Ideas on Cerebellar Involvement in Movement". Cerebellum (London, England) 11.2, pp. 457-487. DOI: 10.1007/s12311-011-0331-9.

Maratos, F. A., S. J. Anderson, A. Hillebrand, K. D. Singh, and G. R. Barnes (2007). "The Spatial Distribution and Temporal Dynamics of Brain Regions Activated during the Perception of Object and Non-Object Patterns". Neurolmage 34.1, pp. 371-383. DOI: 10.1016/j.neuroimage.2006.09.017.

Marchand, W. R., J. N. Lee, J. W. Thatcher, G. W. Thatcher, C. Jensen, and J. Starr (2007). "Motor Deactivation in the Human Cortex and Basal Ganglia". Neurolmage 38.3, pp. 538-548. DOI: $10.1016 / \mathrm{j}$.neuroimage.2007.07.036.

Marco Santello, J. F. S. (1997). "Matching Object Size by Controlling Finger Span and Hand Shape". Somatosensory \& Motor Research 14.3, pp. 203-212. DOI: 10.1080/08990229771060.

Marconi, B., A. Genovesio, A. Battaglia-Mayer, S. Ferraina, S. Squatrito, M. Molinari, F. Lacquaniti, and R. Caminiti (2001). "Eye-Hand Coordination during Reaching. I. Anatomical Relationships between Parietal and Frontal Cortex". Cerebral Cortex 11.6, pp. 513-527. DOI: 10.1093/cercor/11.6.513. 
Marsden, C. D. (1989). "Slowness of Movement in Parkinson's Disease". Movement Disorders 4.S1, S26-S37. DOI: $10.1002 / \mathrm{mds} .870040505$.

Marteniuk, R. G., J. L. Leavitt, C. L. MacKenzie, and S. Athenes (1990). "Functional Relationships between Grasp and Transport Components in a Prehension Task". Human Movement Science 9.2, pp. 149-176. DOI: 10.1016/0167-9457(90) 90025-9.

Marzocchi, N., R. Breveglieri, C. Galletti, and P. Fattori (2008). "Reaching Activity in Parietal Area V6A of Macaque: Eye Influence on Arm Activity or Retinocentric Coding of Reaching Movements?" European Journal of Neuroscience 27.3, pp. 775-789. DOI: 10.1111/j.1460-9568.2008.06021.x.

Mason, C. R., J. E. Gomez, and T. J. Ebner (2001). "Hand Synergies During Reachto-Grasp". Journal of Neurophysiology 86.6, pp. 2896-2910. DOI: $10.1152 /$ jn. 2001.86 .6 .2896 .

Matsumura, M. and K. Kubota (1979). "Cortical Projection to Hand-Arm Motor Area from Post-Arcuate Area in Macaque Monkeys: A Histological Study of Retrograde Transport of Horseradish Peroxidase". Neuroscience Letters 11.3, pp. 241-246. DOI: 10.1016/0304-3940(79)90001-6.

Matsuo, K., C. Kato, T. Okada, T. Moriya, G. H. Glover, and T. Nakai (2003). "Finger Movements Lighten Neural Loads in the Recognition of Ideographic Characters". Cognitive Brain Research 17.2, pp. 263-272. DOI: 10 . 1016 / S09266410(03) 00114-9.

Matt, E., T. Foki, F. Fischmeister, W. Pirker, D. Haubenberger, J. Rath, J. Lehrner, E. Auff, and R. Beisteiner (2017). "Early Dysfunctions of Fronto-Parietal Praxis Networks in Parkinson's Disease". Brain Imaging and Behavior 11.2, pp. 512-525. DOI: $10.1007 / \mathrm{s} 11682-016-9532-7$.

McGuire, L. M. M. and P. N. Sabes (2011). "Heterogeneous Representations in the Superior Parietal Lobule Are Common across Reaches to Visual and Proprioceptive Targets". Journal of Neuroscience 31.18, pp. 6661-6673. DOI: 10.1523/ JNEUROSCI. 2921-10.2011.

McNab, F., G. Leroux, F. Strand, L. Thorell, S. Bergman, and T. Klingberg (2008). "Common and Unique Components of Inhibition and Working Memory: An fMRI, within-Subjects Investigation". Neuropsychologia 46.11, pp. 2668-2682. DOI: 10. 1016/j.neuropsychologia.2008.04.023.

Meck, W. H. and A. M. Benson (2002). "Dissecting the Brain's Internal Clock: How Frontal-Striatal Circuitry Keeps Time and Shifts Attention". Brain and Cognition 48.1, pp. 195-211. DOI: $10.1006 /$ brcg. 2001.1313. 
Meier, J. D., T. N. Aflalo, S. Kastner, and M. S. A. Graziano (2008). "Complex Organization of Human Primary Motor Cortex: A High-Resolution fMRI Study". Journal of Neurophysiology 100.4, pp. 1800-1812. DOI: 10.1152/ jn. 90531. 2008 .

Meister, I., T. Krings, H. Foltys, B. Boroojerdi, M. Müller, R. Töpper, and A. Thron (2004). "Playing Piano in the Mind-an fMRI Study on Music Imagery and Performance in Pianists". Cognitive Brain Research 19.3, pp. 219-228. DOI: 10.1016/j.cogbrainres.2003.12.005.

Meister, I. G., D. Buelte, M. Staedtgen, B. Boroojerdi, and R. Sparing (2009). "The Dorsal Premotor Cortex Orchestrates Concurrent Speech and Fingertapping Movements". European Journal of Neuroscience 29.10, pp. 2074-2082. DOI: 10.1111/ j.1460-9568.2009.06729.x.

Meister, I., T. Krings, H. Foltys, B. Boroojerdi, M. Müller, R. Töpper, and A. Thron (2005). "Effects of Long-term Practice and Task Complexity in Musicians and Nonmusicians Performing Simple and Complex Motor Tasks: Implications for Cortical Motor Organization". Human Brain Mapping 25.3, pp. 345-352. DOI: 10.1002/ hbm.20112.

Menz, V. K., S. Schaffelhofer, and H. Scherberger (2015). "Representation of Continuous Hand and Arm Movements in Macaque Areas M1, F5, and AIP: A Comparative Decoding Study". Journal of Neural Engineering 12.5, p. 056016. DOI: 10.1088/1741-2560/12/5/056016.

Messier, J. and J. F. Kalaska (2000). "Covariation of Primate Dorsal Premotor Cell Activity With Direction and Amplitude During a Memorized-Delay Reaching Task". Journal of Neurophysiology 84.1, pp. 152-165. DOI: 10.1152/jn.2000.84.1. 152 .

Michaels, J. A., B. Dann, R. W. Intveld, and H. Scherberger (2015). "Predicting Reaction Time from the Neural State Space of the Premotor and Parietal Grasping Network". The Journal of Neuroscience 35.32, pp. 11415-11432. DOI: 10.1523/ JNEUROSCI. 1714-15.2015.

- (2018). "Neural Dynamics of Variable Grasp-Movement Preparation in the Macaque Frontoparietal Network". The Journal of Neuroscience 38.25, pp. 5759-5773. DOI: 10.1523/JNEUROSCI . 2557-17.2018.

Milner, T. E., D. W. Franklin, H. Imamizu, and M. Kawato (2007). "Central Control of Grasp: Manipulation of Objects with Complex and Simple Dynamics". Neurolmage 36.2, pp. 388-395. DOI: 10.1016/j.neuroimage.2007.01.057.

Mima, T., N. Sadato, S. Yazawa, T. Hanakawa, H. Fukuyama, Y. Yonekura, and H. Shibasaki (1999). "Brain Structures Related to Active and Passive Finger Move- 
ments in Man". Brain 122.10, pp. 1989-1997. DOI: 10.1093/brain/122.10. 1989 .

Mirabella, G., P. Pani, and S. Ferraina (2011). "Neural Correlates of Cognitive Control of Reaching Movements in the Dorsal Premotor Cortex of Rhesus Monkeys". Journal of Neurophysiology 106.3, pp. 1454-1466. DOI: 10.1152/jn.00995.2010.

Modroño, C., G. Navarrete, A. F. Rodríguez-Hernández, and J. L. González-Mora (2013). "Activation of the Human Mirror Neuron System during the Observation of the Manipulation of Virtual Tools in the Absence of a Visible Effector Limb". Neuroscience Letters 555, pp. 220-224. DOI: 10.1016/j.neulet.2013.09.044. Mohammadi, B., K. Kollewe, D. M. Cole, A. Fellbrich, M. Heldmann, A. Samii, R. Dengler, S. Petri, T. F. Münte, and U. M. Krämer (2015). "Amyotrophic Lateral Sclerosis Affects Cortical and Subcortical Activity Underlying Motor Inhibition and Action Monitoring". Human Brain Mapping 36.8, pp. 2878-2889. DOI: 10.1002/ hbm.22814,

Monaco, S., C. Cavina-Pratesi, A. Sedda, P. Fattori, C. Galletti, and J. C. Culham (2011). "Functional Magnetic Resonance Adaptation Reveals the Involvement of the Dorsomedial Stream in Hand Orientation for Grasping". Journal of Neurophysiology 106.5, pp. 2248-2263. DOI: 10.1152/jn.01069.2010.

Mountcastle, V. B., J. C. Lynch, A. Georgopoulos, H. Sakata, and C. Acuna (1975). "Posterior Parietal Association Cortex of the Monkey: Command Functions for Operations within Extrapersonal Space". Journal of Neurophysiology 38.4, pp. 871908. DOI: $10.1152 /$ jn.1975.38.4.871.

Müller, K., R. Kleiser, F. Mechsner, and R. J. Seitz (2009). "Perceptual Influence on Bimanual Coordination: An fMRI Study". The European Journal of Neuroscience 30.1, pp. 116-124. DOI: $10.1111 / \mathrm{j} .1460-9568.2009 .06802 . \mathrm{x}$.

Murata, A., V. Gallese, M. Kaseda, and H. Sakata (1996). "Parietal Neurons Related to Memory-Guided Hand Manipulation". Journal of Neurophysiology 75.5, pp. 2180-2186. DOI: 10.1152/jn.1996.75.5.2180.

Murata, A., V. Gallese, G. Luppino, M. Kaseda, and H. Sakata (2000). "Selectivity for the Shape, Size, and Orientation of Objects for Grasping in Neurons of Monkey Parietal Area AIP". Journal of Neurophysiology 83.5, pp. 2580-2601. DOI: 10 . $1152 / j n .2000 .83 .5 .2580$

Müri, R. M. (2006). "MRI and fMRI Analysis of Oculomotor Function". Progress in Brain Research. Vol. 151. Elsevier, pp. 503-526. DOI: 10.1016/S0079-6123(05) 51016-1.

Mushiake, H., M. Inase, and J. Tanji (1991). "Neuronal Activity in the Primate Premotor, Supplementary, and Precentral Motor Cortex during Visually Guided 
and Internally Determined Sequential Movements". Journal of Neurophysiology 66.3, pp. 705-718. DOI: $10.1152 /$ jn.1991.66.3.705

Musso, M., C. Weiller, A. Horn, V. Glauche, R. Umarova, J. Hennig, A. Schneider, and M. Rijntjes (2015). "A Single Dual-Stream Framework for Syntactic Computations in Music and Language". Neurolmage 117, pp. 267-283. DOI: 10.1016/ j.neuroimage.2015.05.020.

Naito, E., P. E. Roland, C. Grefkes, H. J. Choi, S. Eickhoff, S. Geyer, K. Zilles, and H. H. Ehrsson (2005). "Dominance of the Right Hemisphere and Role of Area 2 in Human Kinesthesia". Journal of Neurophysiology 93.2, pp. 1020-1034. DOI: $10.1152 / j n .00637 .2004$

Nakagawa, K., S. Kawashima, N. Mizuguchi, and K. Kanosue (2016). "Difference in Activity in the Supplementary Motor Area Depending on Limb Combination of Hand-Foot Coordinated Movements". Frontiers in Human Neuroscience 10. DOI: 10.3389/fnhum. 2016.00499.

Nambu, I., N. Hagura, S. Hirose, Y. Wada, M. Kawato, and E. Naito (2015). “Decoding Sequential Finger Movements from Preparatory Activity in Higher-Order Motor Regions: A Functional Magnetic Resonance Imaging Multi-Voxel Pattern Analysis". European Journal of Neuroscience 42.10, pp. 2851-2859. DOI: 10.1111/ ejn.13063.

Napier, J. R. (1956). "The Prehensile Movements of the Human Hand". The Journal of Bone and Joint Surgery. British volume 38-B.4, pp. 902-913. DOI: 10.1302/ 0301-620X.38B4.902.

Napier, J. R., P. H. Napier, and P. H. Napier (1967). A Handbook of Living Primates: Morphology, Ecology and Behaviour of Nonhuman Primates. Academic P.

Narayanan, N. S. (2016). "Ramping Activity Is a Cortical Mechanism of Temporal Control of Action". Current Opinion in Behavioral Sciences. Time in Perception and Action 8, pp. 226-230. DOI: 10.1016/j.cobeha.2016.02.017

NC3Rs (2017). Non-Human Primate Accommodation, Care and Use.

Nelissen, K., P. A. Fiave, and W. Vanduffel (2018). “Decoding Grasping Movements from the Parieto-Frontal Reaching Circuit in the Nonhuman Primate". Cerebral Cortex 28.4, pp. 1245-1259. DOI: 10.1093/cercor/bhx037.

Nelissen, K. and W. Vanduffel (2011). "Grasping-Related Functional Magnetic Resonance Imaging Brain Responses in the Macaque Monkey". The Journal of Neuroscience 31.22, pp. 8220-8229. DOI: 10.1523/JNEUROSCI.0623-11.2011.

Nenert, R., J. B. Allendorfer, and J. P. Szaflarski (2014). "A Model for Visual Memory Encoding". PLoS ONE 9.10. DOI: 10.1371/journal.pone.0107761. 
Newman, M. E. J. (2006). "Modularity and Community Structure in Networks". Proceedings of the National Academy of Sciences of the United States of America 103.23, pp. 8577-8582. DOI: 10.1073/pnas.0601602103.

Newman, S. D., T. A. Keller, and M. A. Just (2006). "Volitional Control of Attention and Brain Activation in Dual Task Performance". Human Brain Mapping 28.2, pp. 109-117. DOI: $10.1002 / \mathrm{hbm} .20257$.

Nichols, T. E. and A. P. Holmes (2002). "Nonparametric permutation tests for functional neuroimaging: A primer with examples". Human Brain Mapping 15.1, pp. 125. DOI: $10.1002 / \mathrm{hbm} .1058$.

Norman, K. A., S. M. Polyn, G. J. Detre, and J. V. Haxby (2006). "Beyond MindReading: Multi-Voxel Pattern Analysis of fMRI Data". Trends in Cognitive Sciences 10.9, pp. 424-430. DOI: $10.1016 /$ j.tics.2006.07.005.

Norman, L. J., C. O. Carlisi, A. Christakou, A. Cubillo, C. M. Murphy, K. Chantiluke, A. Simmons, V. Giampietro, M. Brammer, D. Mataix-Cols, and K. Rubia (2017). "Shared and Disorder-Specific Task-Positive and Default Mode Network Dysfunctions during Sustained Attention in Paediatric Attention-Deficit/Hyperactivity Disorder and Obsessive/Compulsive Disorder". Neurolmage : Clinical 15, pp. 181-193. DOI: $10.1016 / \mathrm{j} . \mathrm{nicl} .2017 .04 .013$.

Nowik, A. M., P. P. Styrkowiec, and G. Kroliczak (2019). "Manual Grasparatus: A Nifty Tool for Presenting Real Objects in fMRI Research". MethodsX 6, pp. 13531359. DOI: $10.1016 / \mathrm{j} \cdot \operatorname{mex} .2019 .06 .003$.

O'Toole, A. J., F. Jiang, H. Abdi, and J. V. Haxby (2005). "Partially Distributed Representations of Objects and Faces in Ventral Temporal Cortex". Journal of Cognitive Neuroscience 17.4, pp. 580-590. DOI: 10.1162/0898929053467550.

Obeso, J. A., M. C. Rodriguez-Oroz, M. Rodriguez, J. L. Lanciego, J. Artieda, N. Gonzalo, and C. W. Olanow (2000). "Pathophysiology of the Basal Ganglia in Parkinson's Disease". Trends in Neurosciences 23, S8-S19. DOI: 10.1016/S14711931(00)00028-8.

Odeblad, E., B. N. Bhar, and G. Lindström (1956). "Proton Magnetic Resonance of Human Red Blood Cells in Heavy-Water Exchange Experiments". Archives of Biochemistry and Biophysics 63.1, pp. 221-225. DOI: 10.1016/0003-9861(56) 90025-X.

Odeblad, E. and G. Lindstrom (1955). "Some Preliminary Observations on the Proton Magnetic Resonance in Biologic Samples". Acta Radiologica 43.6, pp. 469-476. DOI: $10.3109 / 00016925509172514$. 
Ogawa, K. and F. Imai (2016). "Hand-Independent Representation of Tool-Use Pantomimes in the Left Anterior Intraparietal Cortex". Experimental Brain Research 234.12, pp. 3677-3687. DOI: 10.1007/s00221-016-4765-7.

Ogawa, S., T. M. Lee, A. R. Kay, and D. W. Tank (1990). "Brain Magnetic Resonance Imaging with Contrast Dependent on Blood Oxygenation." Proceedings of the National Academy of Sciences of the United States of America 87.24, pp. 98689872.

Ohlendorf, S., H. Kimmig, V. Glauche, and S. Haller (2007). "Gaze Pursuit, 'Attention Pursuit' and Their Effects on Cortical Activations: Gaze and Attention Pursuit in fMRI". European Journal of Neuroscience 26.7, pp. 2096-2108. DOI: 10.1111/ j.1460-9568.2007.05824.x.

Okano, K. and J. Tanji (1987). "Neuronal Activities in the Primate Motor Fields of the Agranular Frontal Cortex Preceding Visually Triggered and Self-Paced Movement". Experimental Brain Research 66.1, pp. 155-166. DOI: 10.1007/BF00236211.

Oldfield, R. (1971). "The Assessment and Analysis of Handedness: The Edinburgh Inventory". Neuropsychologia 9.1, pp. 97-113. DOI: $10.1016 / 0028-3932$ (71) 90067-4.

Orban, P., P. Peigneux, O. Lungu, G. Albouy, E. Breton, F. Laberenne, H. Benali, P. Maquet, and J. Doyon (2010). "The Multifaceted Nature of the Relationship between Performance and Brain Activity in Motor Sequence Learning". Neurolmage 49.1, pp. 694-702. DOI: 10.1016/j.neuroimage.2009.08.055.

Ortuño, F., F. Guillén-Grima, P. López-García, J. Gómez, and J. Pla (2011). "Functional Neural Networks of Time Perception: Challenge and Opportunity for Schizophrenia Research". Schizophrenia Research 125.2-3, pp. 129-135. DOI: $10.1016 /$ j . schres.2010.10.003.

Pandarinath, C., V. Gilja, C. H. Blabe, P. Nuyujukian, A. A. Sarma, B. L. Sorice, E. N. Eskandar, L. R. Hochberg, J. M. Henderson, and K. V. Shenoy (2015). "Neural Population Dynamics in Human Motor Cortex during Movements in People with ALS". elife 4. Ed. by O. Kiehn, e07436. DOI: 10.7554/eLife.07436.

Parkinson, A., M. McDonagh, and R. Vidyasagar (2009). "Brain Activation in an Involuntary Human Action". Brain Research 1304, pp. 57-65. DOI: $10.1016 / \mathrm{j}$. brainres.2009.09.092.

Parsons, L. M., J. Sergent, D. A. Hodges, and P. T. Fox (2005). "The Brain Basis of Piano Performance". Neuropsychologia. Movement, Action and Consciousness: Toward a Physiology of Intentionality 43.2, pp. 199-215. DOI: $10.1016 / \mathrm{j}$. neuropsychologia.2004.11.007. 
Passarelli, L., M. G. P. Rosa, S. Bakola, M. Gamberini, K. H. Worthy, P. Fattori, and C. Galletti (2018). "Uniformity and Diversity of Cortical Projections to Precuneate Areas in the Macaque Monkey: What Defines Area PGm?" Cerebral Cortex 28.5, pp. 1700-1717. DOI: 10.1093/cercor/bhx067

Paulignan, Y., C. MacKenzie, R. Marteniuk, and M. Jeannerod (1990). "The Coupling of Arm and Finger Movements during Prehension". Experimental Brain Research 79.2, pp. 431-435. DOI: $10.1007 /$ BF00608255.

Pauling, L. and C. D. Coryell (1936). "The Magnetic Properties and Structure of Hemoglobin, Oxyhemoglobin and Carbonmonoxyhemoglobin". Proceedings of the National Academy of Sciences 22.4, pp. 210-216. DOI: 10.1073/pnas .22.4.210. Pecenka, N., A. Engel, and P. E. Keller (2013). "Neural Correlates of Auditory Temporal Predictions during Sensorimotor Synchronization". Frontiers in Human Neuroscience 7. DOI: $10.3389 /$ fnhum.2013.00380.

Pellijeff, A., L. Bonilha, P. S. Morgan, K. McKenzie, and S. R. Jackson (2006). "Parietal Updating of Limb Posture: An Event-Related fMRI Study". Neuropsychologia. Visuomotor Functions of the Posterior Parietal Cortex 44.13, pp. 2685-2690. DOI: 10.1016/j.neuropsychologia.2006.01.009.

Penfield, W. and T. Rasmussen (1950). The Cerebral Cortex of Man; a Clinical Study of Localization of Function. The Cerebral Cortex of Man; a Clinical Study of Localization of Function. Oxford, England: Macmillan, pp. xv, 248.

Pereira, F., T. Mitchell, and M. Botvinick (2009). "Machine Learning Classifiers and fMRI: A Tutorial Overview". Neurolmage. Mathematics in Brain Imaging 45.1, Supplement 1, S199-S209. DOI: 10.1016/j.neuroimage.2008.11.007.

Perenin, M. T. and A. Vighetto (1988). "Optic Ataxia: A Specific Disruption in Visuomotor Mechanisms. I. Different Aspects of the Deficit in Reaching for Objects". Brain: A Journal of Neurology 111 ( Pt 3), pp. 643-674. DOI: 10.1093/brain/ 111.3 .643 .

Pfordresher, P. Q., J. T. Mantell, S. Brown, R. Zivadinov, and J. L. Cox (2014). "Brain Responses to Altered Auditory Feedback during Musical Keyboard Production: An fMRI Study". Brain Research 1556, pp. 28-37. DOI: 10.1016/j . brainres.2014.02.004.

Picard, N. and P. L. Strick (1996). "Motor Areas of the Medial Wall: A Review of Their Location and Functional Activation". Cerebral Cortex (New York, N.Y.: 1991) 6.3, pp. 342-353. DOI: 10.1093/cercor/6.3.342.

Pierrot-Deseilligny, C., F. Gray, and P. Brunet (1986). "Infarcts of Both Inferior Parietal Lobules with Impairment of Visually Guided Eye Movements, Peripheral 
Visual Inattention and Optic Ataxia". Brain: A Journal of Neurology 109 ( Pt 1), pp. 81-97. DOI: $10.1093 /$ brain/109.1.81.

Pimentel, M. A. F., P. Vilela, I. Sousa, and P. Figueiredo (2011). "Localization of the

Hand Motor Area by Arterial Spin Labeling and Blood Oxygen Level-dependent Functional Magnetic Resonance Imaging". Human Brain Mapping 34.1, pp. 96108. DOI: $10.1002 / \mathrm{hbm} .21418$.

Piserchia, V., R. Breveglieri, K. Hadjidimitrakis, F. Bertozzi, C. Galletti, and P. Fattori (2017). "Mixed Body/Hand Reference Frame for Reaching in 3D Space in Macaque Parietal Area PEc". Cerebral Cortex 27.3, pp. 1976-1990. DOI: 10.1093/cercor/ bhw039.

Pitzalis, S., M. I. Sereno, G. Committeri, P. Fattori, G. Galati, A. Tosoni, and C. Galletti (2013). "The Human Homologue of Macaque Area V6A". Neurolmage 82, pp. 517-530. DOI: 10.1016/j.neuroimage.2013.06.026.

Plata Bello, J., C. Modroño, F. Marcano, and J. L. González-Mora (2015). "The Effect of Motor Familiarity during Simple Finger Opposition Tasks". Brain Imaging and Behavior 9.4, pp. 828-838. DOI: 10.1007/s11682-014-9340-x.

Poldrack, R. A., F. W. Sabb, K. Foerde, S. M. Tom, R. F. Asarnow, S. Y. Bookheimer, and B. J. Knowlton (2005). "The Neural Correlates of Motor Skill Automaticity". The Journal of Neuroscience 25.22, pp. 5356-5364. DOI: 10.1523/JNEUROSCI. 3880-04.2005.

Post, M., H. van Duinen, A. Steens, R. Renken, B. Kuipers, N. Maurits, and I. Zijdewind (2007). "Reduced Cortical Activity during Maximal Bilateral Contractions of the Index Finger". Neurolmage 35.1, pp. 16-27. DOI: $10.1016 / j$. neuroimage. 2006.11 .050 .

Pouthas, V., N. George, J.-B. Poline, M. Pfeuty, P.-F. VandeMoorteele, L. Hugueville, A.-M. Ferrandez, S. Lehéricy, D. LeBihan, and B. Renault (2005). "Neural Network Involved in Time Perception: An fMRI Study Comparing Long and Short Interval Estimation". Human Brain Mapping 25.4, pp. 433-441. DOI: 10 . $1002 / \mathrm{hbm}$. 20126.

Pouydebat, E., M. Laurin, P. Gorce, and V. Bels (2008). "Evolution of Grasping among Anthropoids". Journal of Evolutionary Biology 21.6, pp. 1732-1743. DOI: 10.1111/j.1420-9101.2008.01582.x

Prado, J., S. Clavagnier, H. Otzenberger, C. Scheiber, H. Kennedy, and M.-T. Perenin (2005). "Two Cortical Systems for Reaching in Central and Peripheral Vision". Neuron 48.5, pp. 849-858. DOI: 10.1016/j.neuron.2005.10.010.

Prevosto, V., W. Graf, and G. Ugolini (2010). "Cerebellar Inputs to Intraparietal Cortex Areas LIP and MIP: Functional Frameworks for Adaptive Control of Eye Move- 
ments, Reaching, and Arm/Eye/Head Movement Coordination". Cerebral Cortex 20.1, pp. 214-228. DOI: $10.1093 /$ cercor/bhp091.

Rallis, A., K. A. Fercho, T. J. Bosch, and L. A. Baugh (2018). "Getting a Handle on Virtual Tools: An Examination of the Neuronal Activity Associated with Virtual Tool Use". Neuropsychologia 109, pp. 208-221. DOI: 10.1016/j . neuropsychologia. 2017.12 .023 .

Rand, M. K., M. Lemay, L. M. Squire, Y. P. Shimansky, and G. E. Stelmach (2007). "Role of Vision in Aperture Closure Control during Reach-to-Grasp Movements". Experimental Brain Research 181.3, pp. 447-460. DOI: 10.1007/s00221-0070945-9.

Rand, M. K., L. M. Squire, and G. E. Stelmach (2006). "Effect of Speed Manipulation on the Control of Aperture Closure during Reach-to-Grasp Movements". Experimental Brain Research 174.1, pp. 74-85. DOI: 10.1007/s00221-006-0423-9.

Rao, S. M., J. R. Binder, T. A. Hammeke, P. A. Bandettini, J. A. Bobholz, J. A. Frost, B. M. Myklebust, R. D. Jacobson, and J. S. Hyde (1995). "Somatotopic Mapping of the Human Primary Motor Cortex with Functional Magnetic Resonance Imaging". Neurology 45.5, pp. 919-924. DOI: 10.1212/WNL.45.5.919.

Rao, S. M., A. R. Mayer, and D. L. Harrington (2001). "The Evolution of Brain Activation during Temporal Processing". Nature Neuroscience 4.3, pp. 317-323. DOI: $10.1038 / 85191$.

Raos, V., M.-A. Umiltá, V. Gallese, and L. Fogassi (2004). "Functional Properties of Grasping-Related Neurons in the Dorsal Premotor Area F2 of the Macaque Monkey". Journal of Neurophysiology 92.4, pp. 1990-2002. DOI: $10.1152 / \mathrm{jn}$. 00154.2004 .

Raos, V., M.-A. Umiltá, A. Murata, L. Fogassi, and V. Gallese (2006). "Functional Properties of Grasping-Related Neurons in the Ventral Premotor Area F5 of the Macaque Monkey". Journal of Neurophysiology 95.2, pp. 709-729. DOI: 10.1152/ jn.00463.2005.

Ratcliff, G. and G. A. Davies-Jones (1972). "Defective Visual Localization in Focal Brain Wounds". Brain: A Journal of Neurology 95.1, pp. 49-60. DOI: 10.1093/ brain/95.1.49.

Ray Li, C.-s., C. Huang, R. T. Constable, and R. Sinha (2006). "Imaging Response Inhibition in a Stop-Signal Task: Neural Correlates Independent of Signal Monitoring and Post-Response Processing". The Journal of Neuroscience 26.1, pp. 186192. DOI: $10.1523 /$ JNEUROSCI . 3741-05.2006. 
Reed, C. L., S. Shoham, and E. Halgren (2004). "Neural Substrates of Tactile Object Recognition: An fMRI Study". Human Brain Mapping 21.4, pp. 236-246. DOI: 10.1002/hbm.10162.

Rekkas, P., M. Westerveld, P. Skudlarski, J. Zumer, K. Pugh, D. Spencer, and R. Constable (2005). "Neural Correlates of Temporal-Order Judgments versus Those of Spatial-Location: Deactivation of Hippocampus May Facilitate Spatial Performance". Brain and Cognition 59.2, pp. 103-113. DOI: 10.1016/j.bandc.2005. 05.013 .

Rémy, F., N. Wenderoth, K. Lipkens, and S. P. Swinnen (2008). "Acquisition of a New Bimanual Coordination Pattern Modulates the Cerebral Activations Elicited by an Intrinsic Pattern: An fMRI Study". Cortex 44.5, pp. 482-493. DOI: 10 . 1016/j.cortex.2007.07.004.

Reverberi, C., K. Görgen, and J.-D. Haynes (2012). "Compositionality of Rule Representations in Human Prefrontal Cortex". Cerebral Cortex 22.6, pp. 1237-1246. DOI: $10.1093 /$ cercor/bhr200.

Reynaud, E., M. Lesourd, J. Navarro, and F. Osiurak (2016). "On the Neurocognitive Origins of Human Tool Use : A Critical Review of Neuroimaging Data". Neuroscience \& Biobehavioral Reviews 64, pp. 421-437. DOI: 10.1016/j . neubiorev. 2016.03.009.

Rice, N. J., E. Tunik, and S. T. Grafton (2006). "The Anterior Intraparietal Sulcus Mediates Grasp Execution, Independent of Requirement to Update: New Insights from Transcranial Magnetic Stimulation". Journal of Neuroscience 26.31, pp. 8176-8182. DOI: 10.1523/JNEUROSCI.1641-06.2006.

Riecker, A., D. Wildgruber, K. Mathiak, W. Grodd, and H. Ackermann (2003). "Parametric Analysis of Rate-Dependent Hemodynamic Response Functions of Cortical and Subcortical Brain Structures during Auditorily Cued Finger Tapping: A fMRI Study". Neurolmage 18.3, pp. 731-739. DOI: 10.1016/S1053-8119(03)00003$\mathrm{X}$

Ritchie, J. B., D. M. Kaplan, and C. Klein (2019). "Decoding the Brain: Neural Representation and the Limits of Multivariate Pattern Analysis in Cognitive Neuroscience". The British Journal for the Philosophy of Science 70.2, pp. 581-607. DOI: $10.1093 / \mathrm{bjps} / \mathrm{axx} 023$.

Rizzolatti, G., R. Camarda, L. Fogassi, M. Gentilucci, G. Luppino, and M. Matelli (1988). "Functional Organization of Inferior Area 6 in the Macaque Monkey". Experimental Brain Research 71.3, pp. 491-507. DOI: 10.1007/BF00248742.

Rizzolatti, G. and M. Gentilucci (1988). "Motor and Visual-Motor Functions of the Premotor Cortex". Neurobiology of neocortex 42, pp. 269-284. 
Rizzolatti, G. and M. Matelli (2003). "Two Different Streams Form the Dorsal Visual System: Anatomy and Functions". Experimental Brain Research 153.2, pp. 146157. DOI: $10.1007 / \mathrm{s} 00221-003-1588-0$.

Rizzolatti, G., C. Scandolara, M. Matelli, and M. Gentilucci (1981). "Afferent Properties of Periarcuate Neurons in Macaque Monkeys. I. Somatosensory Responses". Behavioural Brain Research 2.2, pp. 125-146. DOI: $10.1016 / 0166-4328(81)$ 90052-8.

Roby-Brami, A., S. Jacobs, N. Bennis, and M. F. Levin (2003). "Hand Orientation for Grasping and Arm Joint Rotation Patterns in Healthy Subjects and Hemiparetic Stroke Patients". Brain Research 969.1-2, pp. 217-229. DOI: $10.1016 /$ S00068993(03) 02334-5.

Rocca, M. A., R. Gatti, F. Agosta, P. Tortorella, E. Riboldi, P. Broglia, and M. Filippi (2007). "Influence of Body Segment Position during In-Phase and Antiphase Hand and Foot Movements: A Kinematic and Functional MRI Study". Human Brain Mapping 28.3, pp. 218-227. DOI: 10.1002/hbm. 20271.

Romaiguère, P., J.-L. Anton, M. Roth, L. Casini, and J.-P. Roll (2003). "Motor and Parietal Cortical Areas Both Underlie Kinaesthesia". Cognitive Brain Research 16.1, pp. 74-82. DOI: 10.1016/S0926-6410(02)00221-5.

Rosenbaum, D. A. (1980). "Human Movement Initiation: Specification of Arm, Direction, and Extent". Journal of Experimental Psychology: General 109.4, pp. 444474. DOI: $10.1037 / 0096-3445.109 .4 .444$.

Rouiller, E. M., J. Tanne, V. Moret, and D. Boussaoud (1999). "Origin of Thalamic Inputs to the Primary, Premotor, and Supplementary Motor Cortical Areas and to Area 46 in Macaque Monkeys: A Multiple Retrograde Tracing Study". Journal of Comparative Neurology 409.1, pp. 131-152. DOI: 10 .1002/(SICI ) 1096 9861(19990621) 409:1<131::AID-CNE10>3.0.C0;2-A.

Rowe, J., L. Hughes, D. Eckstein, and A. M. Owen (2008). "Rule-Selection and Action-Selection Have a Shared Neuroanatomical Basis in the Human Prefrontal and Parietal Cortex". Cerebral Cortex 18.10, pp. 2275-2285. DOI: 10.1093/ cercor/bhm249.

Rumiati, R. I., P. H. Weiss, T. Shallice, G. Ottoboni, J. Noth, K. Zilles, and G. R. Fink (2004). "Neural Basis of Pantomiming the Use of Visually Presented Objects". Neurolmage 21.4, pp. 1224-1231. DOI: 10.1016/j.neuroimage.2003.11.017. Rushworth, M. F. S., P. D. Nixon, and R. E. Passingham (1997). "Parietal Cortex and Movement I. Movement Selection and Reaching". Experimental brain research 117.2, pp. 292-310. 
Rushworth, M., K. A. Hadland, T. Paus, and P. K. Sipila (2002). "Role of the Human Medial Frontal Cortex in Task Switching: A Combined fMRI and TMS Study". Journal of Neurophysiology 87.5, pp. 2577-2592. DOI: 10.1152/ jn.2002.87. 5.2577

Sakai, K., O. Hikosaka, R. Takino, S. Miyauchi, M. Nielsen, and T. Tamada (2000). "What and When: Parallel and Convergent Processing in Motor Control". The Journal of Neuroscience 20.7, pp. 2691-2700. DOI: 10.1523/JNEUROSCI . 20 07-02691.2000.

Sakata, H., M. Taira, A. Murata, and S. Mine (1995). "Neural Mechanisms of Visual Guidance of Hand Action in the Parietal Cortex of the Monkey". Cerebral Cortex 5.5, pp. 429-438. DOI: $10.1093 /$ cercor/5.5.429

Saling, M., S. Mescheriakov, E. Molokanova, G. E. Stelmach, and M. Berger (1996). "Grip Reorganization during Wrist Transport: The Influence of an Altered Aperture". Experimental Brain Research 108.3, pp. 493-500. DOI: 10.1007/BF00227272. Sandberg, K., L. M. Andersen, and M. Overgaard (2014). "Using Multivariate Decoding to Go beyond Contrastive Analyses in Consciousness Research". Frontiers in Psychology 5. DOI: 10.3389/fpsyg.2014.01250.

Sangole, A. P. and M. F. Levin (2008). "Palmar Arch Dynamics during Reach-toGrasp Tasks". Experimental Brain Research 190.4, pp. 443-452. DOI: 10.1007/ s00221-008-1486-6.

Santandrea, E., R. Breveglieri, A. Bosco, C. Galletti, and P. Fattori (2018). "Preparatory Activity for Purposeful Arm Movements in the Dorsomedial Parietal Area V6A: Beyond the Online Guidance of Movement". Scientific Reports 8.1, p. 6926. DOI: 10.1038/s41598-018-25117-0.

Santello, M. and J. F. Soechting (1998). "Gradual Molding of the Hand to Object Contours". Journal of Neurophysiology 79.3, pp. 1307-1320. DOI: 10.1152/jn. 1998.79.3.1307.

Sayegh, P. F., K. M. Hawkins, B. Neagu, J. D. Crawford, K. L. Hoffman, and L. E. Sergio (2014). "Decoupling the Actions of the Eyes from the Hand Alters Beta and Gamma Synchrony within SPL". Journal of Neurophysiology 111.11, pp. 22102221. DOI: $10.1152 / \mathrm{jn}$.00793.2013.

Schaffelhofer, S., A. Agudelo-Toro, and H. Scherberger (2015). "Decoding a Wide Range of Hand Configurations from Macaque Motor, Premotor, and Parietal Cortices". The Journal of Neuroscience 35.3, pp. 1068-1081. DOI: 10.1523/JNEUROSCI . 3594-14.2015. 
Schaffelhofer, S. and H. Scherberger (2016). "Object Vision to Hand Action in Macaque Parietal, Premotor, and Motor Cortices". elife 5, e15278. DOI: 10. 7554/eLife.15278.

Scheidt, R. A., J. L. Zimbelman, N. M. G. Salowitz, A. J. Suminski, K. M. Mosier, J. Houk, and L. Simo (2012). "Remembering Forward: Neural Correlates of Memory and Prediction in Human Motor Adaptation". Neurolmage 59.1, pp. 582-600. DOI: 10.1016/j.neuroimage.2011.07.072.

Schieber, M. H. (1999). "Somatotopic Gradients in the Distributed Organization of the Human Primary Motor Cortex Hand Area: Evidence from Small Infarcts". Experimental Brain Research 128.1, pp. 139-148. DOI: 10.1007/s002210050829. Schreiber, T. (2000). "Measuring Information Transfer". Physical Review Letters 85.2, pp. 461-464. DOI: 10.1103/PhysRevLett.85.461.

Schubotz, R. I. and D. Y. von Cramon (2001). "Interval and Ordinal Properties of Sequences Are Associated with Distinct Premotor Areas". Cerebral Cortex 11.3, pp. 210-222. DOI: $10.1093 /$ cercor/11.3.210.

- (2003). "Functional-Anatomical Concepts of Human Premotor Cortex: Evidence from fMRI and PET Studies". Neurolmage 20, S120-S131. DOI: $10.1016 / \mathrm{j}$. neuroimage.2003.09.014.

Schulte, T., E. M. Müller-Oehring, S. Vinco, F. Hoeft, A. Pfefferbaum, and E. V. Sullivan (2009). "Double Dissociation Between Action-Driven and Perception-Driven Conflict Resolution Invoking Anterior versus Posterior Brain Systems". Neurolmage 48.2, pp. 381-390. DOI: $10.1016 /$ j. neuroimage. 2009.06.058.

Schwartze, M., K. Rothermich, and S. A. Kotz (2012). "Functional Dissociation of Pre-SMA and SMA-Proper in Temporal Processing". Neurolmage 60.1, pp. 290298. DOI: $10.1016 / \mathrm{j}$. neuroimage.2011.11.089.

Scott, S. H., L. E. Sergio, and J. F. Kalaska (1997). "Reaching Movements With Similar Hand Paths but Different Arm Orientations. II. Activity of Individual Cells in Dorsal Premotor Cortex and Parietal Area 5". Journal of Neurophysiology 78.5, pp. 2413-2426. DOI: 10.1152/jn.1997.78.5.2413.

Sebastian, A., B. Gerdes, B. Feige, S. Klöppel, T. Lange, A. Philipsen, L. Tebartz van Elst, K. Lieb, and O. Tüscher (2012). "Neural Correlates of Interference Inhibition, Action Withholding and Action Cancelation in Adult ADHD". Psychiatry Research: Neuroimaging 202.2, pp. 132-141. DOI: $10.1016 /$ j.pscychresns.2012.02. 010.

Seeley, W. W., V. Menon, A. F. Schatzberg, J. Keller, G. H. Glover, H. Kenna, A. L. Reiss, and M. D. Greicius (2007). "Dissociable Intrinsic Connectivity Networks 
for Salience Processing and Executive Control". Journal of Neuroscience 27.9, pp. 2349-2356. DOI: 10.1523/JNEUROSCI.5587-06.2007.

Seger, C. A., B. J. Spiering, A. G. Sares, S. I. Quraini, C. Alpeter, J. David, and M. H. Thaut (2013). "Corticostriatal Contributions to Musical Expectancy Perception". Journal of Cognitive Neuroscience 25.7, pp. 1062-1077. DOI: 10.1162/jocn_a_ 00371 .

Seidler, R., D. Noll, and G. Thiers (2004). "Feedforward and Feedback Processes in Motor Control". Neurolmage 22.4, pp. 1775-1783. DOI: 10.1016/j .neuroimage. 2004.05 .003 .

Shibasaki, H. and M. Hallett (2006). "What Is the Bereitschaftspotential?" Clinical Neurophysiology 117.11, pp. 2341-2356. DOI: $10.1016 /$ j.clinph.2006.04. 025 .

Shikata, E., F. Hamzei, V. Glauche, M. Koch, C. Weiller, F. Binkofski, and C. Büchel (2003). "Functional Properties and Interaction of the Anterior and Posterior Intraparietal Areas in Humans". European Journal of Neuroscience 17.5, pp. 11051110. DOI: $10.1046 / \mathrm{j} .1460-9568.2003 .02540 . \mathrm{x}$.

Shmuelof, L. and E. Zohary (2006). "A Mirror Representation of Others' Actions in the Human Anterior Parietal Cortex". Journal of Neuroscience 26.38, pp. 97369742. DOI: 10.1523/JNEUROSCI. 1836-06.2006.

Simon, O., J.-F. Mangin, L. Cohen, D. Le Bihan, and S. Dehaene (2002). "Topographical Layout of Hand, Eye, Calculation, and Language-Related Areas in the Human Parietal Lobe". Neuron 33.3, pp. 475-487. DOI: 10.1016/S08966273(02) 00575-5.

Simon, S. R., M. Meunier, L. Piettre, A. M. Berardi, C. M. Segebarth, and D. Boussaoud (2002). "Spatial Attention and Memory Versus Motor Preparation: Premotor Cortex Involvement as Revealed by fMRI". Journal of Neurophysiology 88.4, pp. 2047-2057. DOI: 10.1152/jn.2002.88.4.2047.

Smeets, J. B., L. O. Wijdenes, and E. Brenner (2016). "Movement Adjustments Have Short Latencies Because There Is No Need to Detect Anything". Motor Control 20.2, pp. 137-148. DOI: 10.1123/mc.2014-0064.

Snyder, L. H., A. P. Batista, and R. A. Andersen (1997). "Coding of Intention in the Posterior Parietal Cortex". Nature 386.6621, pp. 167-170. DOI: 10.1038/ $386167 \mathrm{a} 0$.

- (1998). "Change in Motor Plan, without a Change in the Spatial Locus of Attention, Modulates Activity in Posterior Parietal Cortex". Journal of Neurophysiology 79.5, pp. 2814-2819. DOI: 10.1152/jn.1998.79.5.2814 
Solesio-Jofre, E., L. Serbruyns, D. G. Woolley, D. Mantini, I. A. M. Beets, and S. P. Swinnen (2014). "Aging Effects on the Resting State Motor Network and Interlimb Coordination". Human Brain Mapping 35.8, pp. 3945-3961. DOI: 10.1002/hbm. 22450.

Song, J.-H. and R. M. McPeek (2010). "Roles of Narrow- and Broad-Spiking Dorsal Premotor Area Neurons in Reach Target Selection and Movement Production". Journal of Neurophysiology 103.4, pp. 2124-2138. DOI: 10.1152 / jn . 00238 . 2009 .

Stark, E., I. Asher, and M. Abeles (2007). "Encoding of Reach and Grasp by Single Neurons in Premotor Cortex Is Independent of Recording Site". Journal of Neurophysiology 97.5, pp. 3351-3364. DOI: 10.1152/jn.01328.2006.

Stelzer, J., Y. Chen, and R. Turner (2013). "Statistical Inference and Multiple Testing Correction in Classification-Based Multi-Voxel Pattern Analysis (MVPA): Random Permutations and Cluster Size Control". Neurolmage 65, pp. 69-82. DOI: 10 . 1016/j.neuroimage.2012.09.063.

Stoeckel, M. C., B. Pollok, A. Schnitzler, and R. J. Seitz (2007). "Studying the Human Somatosensory Hand Area: A New Way to Compare fMRI and MEG". Journal of Neuroscience Methods 164.2, pp. 280-291. DOI: 10.1016/j . jneumeth. 2007. 05.016 .

Striemer, C. L., P. A. Chouinard, and M. A. Goodale (2011). "Programs for Action in Superior Parietal Cortex: A Triple-Pulse TMS Investigation". Neuropsychologia 49.9, pp. 2391-2399. DOI: 10.1016/j.neuropsychologia.2011.04.015.

Striemer, C. L., P. A. Chouinard, M. A. Goodale, and S. de Ribaupierre (2015). "Overlapping Neural Circuits for Visual Attention and Eye Movements in the Human Cerebellum". Neuropsychologia 69, pp. 9-21. DOI:10.1016/j . neuropsychologia. 2015.01.024.

Sturm, W., B. Schmenk, B. Fimm, K. Specht, S. Weis, A. Thron, and K. Willmes (2006). "Spatial Attention: More than Intrinsic Alerting?" Experimental Brain Research 171.1, pp. 16-25. DOI: 10.1007/s00221-005-0253-1.

Suminski, A. J., S. M. Rao, K. M. Mosier, and R. A. Scheidt (2007). "Neural and Electromyographic Correlates of Wrist Posture Control". Journal of Neurophysiology 97.2, pp. 1527-1545. DOI: 10.1152/jn.01160.2006.

Sustaita, D., E. Pouydebat, A. Manzano, V. Abdala, F. Hertel, and A. Herrel (2013). "Getting a Grip on Tetrapod Grasping: Form, Function, and Evolution". Biological Reviews 88.2, pp. 380-405. DOI: 10.1111 /brv.12010.

Suzuki, D. T. (1959). Zen and Japanese Culture. Princeton University Press. DOI: $10.2307 / \mathrm{j} \cdot \operatorname{ctvc} 77449$. 
Swinnen, S. P., S. Vangheluwe, J. Wagemans, J. P. Coxon, D. J. Goble, A. Van Impe, S. Sunaert, R. Peeters, and N. Wenderoth (2010). "Shared Neural Resources between Left and Right Interlimb Coordination Skills: The Neural Substrate of Abstract Motor Representations". Neurolmage 49.3, pp. 2570-2580. DOI: 10 . 1016/j.neuroimage.2009.10.052.

Szameitat, A. J., S. Shen, A. Conforto, and A. Sterr (2012). "Cortical Activation during Executed, Imagined, Observed, and Passive Wrist Movements in Healthy Volunteers and Stroke Patients". Neurolmage 62.1, pp. 266-280. DOI: $10.1016 /$ j. neuroimage.2012.05.009.

Taira, M., S. Mine, A. P. Georgopoulos, A. Murata, and H. Sakata (1990). "Parietal Cortex Neurons of the Monkey Related to the Visual Guidance of Hand Movement". Experimental Brain Research 83.1, pp. 29-36. DOI: 10.1007/BF00232190.

Tanné-Gariépy, J., D. Boussaoud, and E. M. Rouiller (2002a). "Projections of the Claustrum to the Primary Motor, Premotor, and Prefrontal Cortices in the Macaque Monkey". The Journal of Comparative Neurology 454.2, pp. 140-157. DOI: 10 . $1002 /$ cne. 10425 .

Tanné-Gariépy, J., E. M. Rouiller, and D. Boussaoud (2002b). "Parietal Inputs to Dorsal versus Ventral Premotor Areas in the Macaque Monkey: Evidence for Largely Segregated Visuomotor Pathways". Experimental Brain Research 145.1, pp. 91103. DOI: $10.1007 / \mathrm{s} 00221-002-1078-9$.

Tanne, J., D. Boussaoud, N. Boyer-Zeller, and E. Rouiller (1995). "Parietal Inputs to Physiologically Defined Regions of Dorsal Premotor Cortex in the Macaque Monkey". Eur J Neurosci 8, pp. 195-201.

Tanné, J., D. Boussaoud, N. Boyer-Zeller, and E. M. Rouiller (1995). "Direct Visual Pathways for Reaching Movements in the Macaque Monkey". NeuroReport 7.1, p. 267.

Thach, W. T. (2014). "Does the Cerebellum Initiate Movement?" The Cerebellum 13.1, pp. 139-150. DOI: $10.1007 / \mathrm{s} 12311-013-0506-7$

Thoenissen, D., K. Zilles, and I. Toni (2002). “Differential Involvement of Parietal and Precentral Regions in Movement Preparation and Motor Intention". The Journal of Neuroscience 22.20, pp. 9024-9034. DOI: 10 . 1523/ JNEUROSCI . 22 - 20 09024.2002 .

Thulborn, K. R., J. C. Waterton, P. M. Matthews, and G. K. Radda (1982). “Oxygenation Dependence of the Transverse Relaxation Time of Water Protons in Whole Blood at High Field". Biochimica et Biophysica Acta (BBA) - General Subjects 714.2, pp. 265-270. DOI: $10.1016 / 0304-4165$ (82)90333-6. 
Tillmann, B., P. Janata, and J. J. Bharucha (2003). "Activation of the Inferior Frontal Cortex in Musical Priming". Cognitive Brain Research 16.2, pp. 145-161. DOI: 10.1016/S0926-6410(02) 00245-8.

Timm, N. H. (2002). Applied Multivariate Analysis. Springer Texts in Statistics. New York: Springer-Verlag. DOI: $10.1007 / \mathrm{b} 98963$

Tipples, J., V. Brattan, and P. Johnston (2013). "Neural Bases for Individual Differences in the Subjective Experience of Short Durations (Less than 2 Seconds)". PLOS ONE 8.1. DOI: 10.1371/journal.pone.0054669.

Todd, M. T., L. E. Nystrom, and J. D. Cohen (2013). "Confounds in Multivariate Pattern Analysis: Theory and Rule Representation Case Study". Neurolmage 77, pp. 157-165. DOI: 10.1016/j.neuroimage.2013.03.039

Toiviainen, P., V. Alluri, E. Brattico, M. Wallentin, and P. Vuust (2014). "Capturing the Musical Brain with Lasso: Dynamic Decoding of Musical Features from fMRI Data". Neurolmage 88, pp. 170-180. DOI: $10.1016 / \mathrm{j}$. neuroimage.2013.11. 017.

Tong, F. and M. S. Pratte (2011). "Decoding Patterns of Human Brain Activity". Annual Review of Psychology 63.1, pp. 483-509. DOI: 10.1146/annurev-psych120710-100412.

Toro, R., P. T. Fox, and T. Paus (2008). "Functional Coactivation Map of the Human Brain". Cerebral Cortex (New York, NY) 18.11, pp. 2553-2559. DOI: 10.1093/ cercor/bhn014.

Tosoni, A., G. Galati, G. L. Romani, and M. Corbetta (2008). "Sensory-Motor Mechanisms in Human Parietal Cortex Underlie Arbitrary Visual Decisions". Nature Neuroscience 11.12, pp. 1446-1453. DOI: $10.1038 / \mathrm{nn} .2221$.

Touroutoglou, A., M. Hollenbeck, B. C. Dickerson, and L. F. Barrett (2012). "Dissociable Large-Scale Networks Anchored in the Right Anterior Insula Subserve Affective Experience and Attention". Neuroimage 60.4, pp. 1947-1958. DOI: 10. 1016/j.neuroimage.2012.02.012.

Touvet, F., A. Roby-Brami, M. A. Maier, and S. Eskiizmirliler (2014). "Grasp: Combined Contribution of Object Properties and Task Constraints on Hand and Finger Posture". Experimental Brain Research 232.10, pp. 3055-3067. DOI: $10.1007 /$ s00221-014-3990-1.

Tsubomi, H., T. Ikeda, T. Hanakawa, N. Hirose, H. Fukuyama, and N. Osaka (2009). "Connectivity and Signal Intensity in the Parieto-Occipital Cortex Predicts Topdown Attentional Effect in Visual Masking: An fMRI Study Based on Individual Differences". Neurolmage 45.2, pp. 587-597. DOI: $10.1016 / \mathrm{j}$. neuroimage . 2008.11.028. 
Tunik, E., S. H. Frey, and S. T. Grafton (2005). "Virtual Lesions of the Anterior Intraparietal Area Disrupt Goal-Dependent on-Line Adjustments of Grasp". Nature Neuroscience 8.4, p. 505. DOI: $10.1038 / \mathrm{nn} 1430$.

Tzourio-Mazoyer, N., B. Landeau, D. Papathanassiou, F. Crivello, O. Etard, N. Delcroix, B. Mazoyer, and M. Joliot (2002). "Automated Anatomical Labeling of Activations in SPM Using a Macroscopic Anatomical Parcellation of the MNI MRI Single-Subject Brain". Neurolmage 15.1, pp. 273-289. DOI: $10.1006 / \mathrm{nimg}$. 2001.0978.

Ugolini, G., V. Prevosto, and W. Graf (2019). "Ascending Vestibular Pathways to Parietal Areas MIP and LIPv and Efference Copy Inputs from the Medial Reticular Formation: Functional Frameworks for Body Representations Updating and Online Movement Guidance". European Journal of Neuroscience 50.6, pp. 2988-3013. DOI: $10.1111 /$ ejn.14426.

Ullén, F., H. Forssberg, and H. H. Ehrsson (2003). "Neural Networks for the Coordination of the Hands in Time". Journal of Neurophysiology 89.2, pp. 1126-1135. DOI: $10.1152 /$ jn.00775.2002.

Ullsperger, M. and D. Y. von Cramon (2003). "Error Monitoring Using External Feedback: Specific Roles of the Habenular Complex, the Reward System, and the Cingulate Motor Area Revealed by Functional Magnetic Resonance Imaging". The Journal of Neuroscience 23.10, pp. 4308-4314. DOI: 10.1523/ JNEUROSCI . 23 $10-04308.2003$.

Umilta, M. A., T. Brochier, R. L. Spinks, and R. N. Lemon (2007). "Simultaneous Recording of Macaque Premotor and Primary Motor Cortex Neuronal Populations Reveals Different Functional Contributions to Visuomotor Grasp". Journal of Neurophysiology 98.1, pp. 488-501. DOI: 10.1152/jn.01094.2006

Umiltà, M. A., L. Escola, I. Intskirveli, F. Grammont, M. Rochat, F. Caruana, A. Jezzini, V. Gallese, and G. Rizzolatti (2008). "When Pliers Become Fingers in the Monkey Motor System". Proceedings of the National Academy of Sciences 105.6, pp. 2209-2213. DOI: 10.1073/pnas.0705985105.

Vaidya, A. R., M. S. Pujara, M. Petrides, E. A. Murray, and L. K. Fellows (2019). "Lesion Studies in Contemporary Neuroscience". Trends in Cognitive Sciences 23.8, pp. 653-671. DOI: 10.1016/j.tics.2019.05.009.

Verhagen, L., H. C. Dijkerman, W. P. Medendorp, and I. Toni (2012). "Cortical Dynamics of Sensorimotor Integration during Grasp Planning". Journal of Neuroscience 32.13, pp. 4508-4519. DOI: 10.1523/JNEUROSCI.5451-11.2012.

Vesia, M., D. A. Bolton, G. Mochizuki, and W. R. Staines (2013). "Human Parietal and Primary Motor Cortical Interactions Are Selectively Modulated during the 
Transport and Grip Formation of Goal-Directed Hand Actions". Neuropsychologia 51.3, pp. 410-417. DOI: $10.1016 /$ j.neuropsychologia.2012.11.022.

Vesia, M. and J. D. Crawford (2012). "Specialization of Reach Function in Human Posterior Parietal Cortex". Experimental Brain Research 221.1, pp. 1-18. DOI: $10.1007 / \mathrm{s} 00221-012-3158-9$.

Vesia, M., J. A. Monteon, L. E. Sergio, and J. D. Crawford (2006). "Hemispheric Asymmetry in Memory-Guided Pointing During Single-Pulse Transcranial Magnetic Stimulation of Human Parietal Cortex". Journal of Neurophysiology 96.6, pp. 3016-3027. DOI: 10.1152/jn.00411.2006.

Vesia, M., S. L. Prime, X. Yan, L. E. Sergio, and J. D. Crawford (2010). "Specificity of Human Parietal Saccade and Reach Regions during Transcranial Magnetic Stimulation". Journal of Neuroscience 30.39, pp. 13053-13065. DOI: 10.1523/ JNEUROSCI . 1644-10.2010.

Vesia, M., X. Yan, D. Y. Henriques, L. E. Sergio, and J. D. Crawford (2008). “Transcranial Magnetic Stimulation Over Human Dorsal-Lateral Posterior Parietal Cortex Disrupts Integration of Hand Position Signals Into the Reach Plan". Journal of Neurophysiology 100.4, pp. 2005-2014. DOI: 10.1152/jn.90519.2008.

Wallace, S. A. and D. L. Weeks (1988). "Temporal Constraints in the Control of Prehensile Movement". Journal of Motor Behavior 20.2, pp. 81-105. DOI: 10 . 1080/00222895.1988.10735435.

Walter, W. G., R. Cooper, V. J. Aldridge, W. C. McCALLUM, and A. L. Winter (1964). "Contingent Negative Variation : An Electric Sign of Sensori-Motor Association and Expectancy in the Human Brain". Nature 203.4943, pp. 380-384. DOI: 10.1038/203380a0.

Weinrich, M. and S. P. Wise (1982). "The Premotor Cortex of the Monkey". Journal of Neuroscience 2.9, pp. 1329-1345. DOI: 10.1523/JNEUROSCI.02-09-01329. 1982 .

Wenderoth, N., F. Debaere, S. Sunaert, and S. P. Swinnen (2005). "The Role of Anterior Cingulate Cortex and Precuneus in the Coordination of Motor Behaviour". European Journal of Neuroscience 22.1, pp. 235-246. DOI: $10.1111 / \mathrm{j} .1460-$ 9568.2005.04176.x.

Werner, S. and U. Noppeney (2011). "The Contributions of Transient and Sustained Response Codes to Audiovisual Integration". Cerebral Cortex 21.4, pp. 920-931. DOI: $10.1093 /$ cercor/bhq161.

Whishaw, I. Q. and J. M. Karl (2014). "The Contribution of the Reach and the Grasp to Shaping Brain and Behaviour". Canadian Journal of Experimental Psy- 
chology/Revue canadienne de psychologie expérimentale 68.4, pp. 223-235. DOI: $10.1037 /$ cep0000042.

Wiener, M., P. Turkeltaub, and H. B. Coslett (2010). "The Image of Time: A

Voxel-Wise Meta-Analysis". Neurolmage 49.2, pp. 1728-1740. DOI: $10.1016 /$ j.neuroimage.2009.09.064.

Wing, E. A., M. Ritchey, and R. Cabeza (2015). "Reinstatement of Individual Past Events Revealed by the Similarity of Distributed Activation Patterns during Encoding and Retrieval". Journal of cognitive neuroscience 27.4, pp. 679-691. DOI: 10.1162/jocn_a_00740.

Winges, S. A., D. J. Weber, and M. Santello (2003). "The Role of Vision on Hand Preshaping during Reach to Grasp". Experimental Brain Research 152.4, pp. 489498. DOI: $10.1007 / \mathrm{s} 00221-003-1571-9$.

Wittmann, M., D. S. Leland, and M. P. Paulus (2007). "Time and Decision Making:

Differential Contribution of the Posterior Insular Cortex and the Striatum during a Delay Discounting Task". Experimental Brain Research 179.4, pp. 643-653. DOI: $10.1007 / \mathrm{s} 00221-006-0822-\mathrm{y}$.

Wittmann, M., A. N. Simmons, J. L. Aron, and M. P. Paulus (2010). "Accumulation of Neural Activity in the Posterior Insula Encodes the Passage of Time". Neuropsychologia 48.10, p. 3110. DOI: 10.1016/j.neuropsychologia.2010.06.023

Wolfensteller, U., R. I. Schubotz, and D. Y. von Cramon (2007). "Understanding Non-Biological Dynamics with Your Own Premotor System". Neurolmage. Cortical Control of Higher Motor Cognition 36, T33-T43. DOI: $10.1016 / \mathrm{j}$. neuroimage. 2007.03 .040 .

Wolpert, D. M., S. J. Goodbody, and M. Husain (1998). "Maintaining Internal Representations: The Role of the Human Superior Parietal Lobe". Nature Neuroscience 1.6, pp. 529-533. DOI: $10.1038 / 2245$.

Woolgar, A., P. Golland, and S. Bode (2014). "Coping with Confounds in Multivoxel Pattern Analysis: What Should We Do about Reaction Time Differences? A Comment on Todd, Nystrom \& Cohen 2013". Neurolmage 98, pp. 506-512. DOI: $10.1016 / \mathrm{j}$.neuroimage.2014.04.059.

Wu, C.-T., D. Weissman, K. Roberts, and M. Woldorff (2007). "The Neural Circuitry Underlying the Executive Control of Auditory Spatial Attention". Brain research 1134.1, pp. 187-198. DOI: 10.1016/j.brainres.2006.11.088.

Yamagishi, N., N. Goda, D. E. Callan, S. J. Anderson, and M. Kawato (2005). "Attentional Shifts towards an Expected Visual Target Alter the Level of AlphaBand Oscillatory Activity in the Human Calcarine Cortex". Cognitive Brain Research 25.3, pp. 799-809. DOI: 10.1016/j.cogbrainres.2005.09.006. 
Yang, G. and C. ladecola (1996). "Glutamate Microinjections in Cerebellar Cortex Reproduce Cerebrovascular Effects of Parallel Fiber Stimulation". American Journal of Physiology-Regulatory, Integrative and Comparative Physiology 271.6, R1568R1575. DOI: 10.1152/ajpregu.1996.271.6.R1568.

Yang, Z., T. Jackson, and C. Huang (2016). "Neural Activation during Anticipation of Near Pain-Threshold Stimulation among the Pain-Fearful". Frontiers in Neuroscience 10. DOI: $10.3389 /$ fnins.2016.00342.

Ye, Z., M. Kutas, M. St. George, M. I. Sereno, F. Ling, and T. F. Münte (2012). "Rearranging the World: Neural Network Supporting the Processing of Temporal Connectives". Neurolmage 59.4, pp. 3662-3667. DOI: 10.1016/j . neuroimage. 2011.11.039.

Yuan, H., C. Perdoni, L. Yang, and B. He (2011). "Differential Electrophysiological Coupling for Positive and Negative BOLD Responses during Unilateral Hand Movements". The Journal of Neuroscience 31.26, pp. 9585-9593. DOI: 10.1523/ JNEUROSCI . 5312-10.2011.

Yuste, R. (2015). "From the Neuron Doctrine to Neural Networks". Nature Reviews Neuroscience 16.8, pp. 487-497. DOI: 10.1038/nrn3962.

Zatorre, R. J. and P. Belin (2001). "Spectral and Temporal Processing in Human Auditory Cortex". Cerebral Cortex 11.10, pp. 946-953. DOI: 10.1093/cercor/ 11.10 .946

Zhang, T., T. Liu, F. Li, M. Li, D. Liu, R. Zhang, H. He, P. Li, J. Gong, C. Luo, D. Yao, and P. Xu (2016). "Structural and Functional Correlates of Motor Imagery BCl Performance: Insights from the Patterns of Fronto-Parietal Attention Network". Neurolmage 134, pp. 475-485. DOI: 10.1016/j.neuroimage.2016.04.030.

Zoccatelli, G., A. Beltramello, F. Alessandrini, F. B. Pizzini, and G. Tassinari (2010). "Word and Position Interference in Stroop Tasks: A Behavioral and fMRI Study". Experimental Brain Research 207.1, pp. 139-147. DOI: 10.1007/s00221-0102433-x 


\title{
A. Additional Publications
}

\author{
A.1. Brendel, A., Greulich, R.S., Niederman, F., \\ Trang, S., 2020. Towards a Greater \\ Diversity of Replication Studies. AIS \\ Transactions on Replication Research 6.
}

This work was a collaboration with Prof. Brendel and first authorship is shared. It includes an extensive literature review of publication studies in neuroscience and categorizes the observed types of replication studies. Two new types of replication study were found within the observed literature corpus, with further discussion yielding a third possible type. 


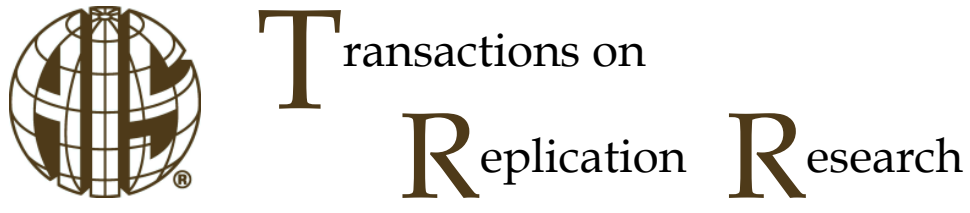

\section{Towards a Greater Diversity of Replication Studies}

\author{
Alfred Benedikt Brendel \\ University of Goettingen and \\ Technische Universität Dresden \\ Alfred_benedikt.brendel@tu-dresden.de \\ Fred Niederman \\ Saint Louis University \\ Fred.niederman@slu.edu
}

\author{
R. Stefan Greulich \\ Deutsches Primatenzentrum GmbH \\ SGreulich@dpz.eu \\ Simon Trang \\ University of Goettingen \\ Simon.trang@wiwi.uni-goettingen.de
}

Abstract:

The replication of existing knowledge (e.g., previous study results) stands as an essential research practice across all science disciplines. Despite the importance of replication, the scarcity of replication studies is commonly criticized in business, management, and information system (IS) research. Therefore, efforts have already been made to facilitate replication research in the IS community, such as establishing conference tracks and journals focusing on publishing replication studies and providing guidelines on how and why to conduct replication research. Nonetheless, the perception of replication research remains unchanged, describing it as mundane.

Therefore, in this issues and opinions article, we will explore how replication research could be made more appealing by diversifying the categories of replication studies. In this regard, we looked at replication in neuroscience, eliciting two new replication study categories: 'transfer' and 'method.' Additionally, through extensive discussion with other IS scholars, we added one more replication category, 'comparison.' We hope that this diversification will attract more researchers and also show the potential replication research holds.

Keywords: Replication Research, Replication Study Categories, NeurolS, Neuroscience, Literature Review

The manuscript was received 02/09/2020 and was with the authors 2 months for 2 revisions. 


\section{Introduction}

Replication research, being the replication of research results, stands as an important research practice across all science disciplines (Dennis \& Valacich, 2014; Gómez et al., 2010; King, 1995). The replication of results converts tentative belief into accepted, tested, and dependable knowledge (Berthon et al., 2002). Hence, replication is a powerful concept for scientific progress by reinforcing the foundations for new advancements and discoveries (Schmidt, 2009).

Despite its importance, the rarity of replication research remains a major weak point and is commonly criticized in business, management, and IS research (Berthon et al., 2002; Dennis \& Valacich, 2014; Hart \& Gregor, 2012). Other disciplines are also struggling with a lack of replication research. In this context, Baker (2016) investigated reproducibility in various disciplines (chemistry, biology, physics, engineering, medicine, and environmental studies) by questioning over 1,500 researchers. Around $90 \%$ of researchers perceive science to have at least a slight reproducibility crisis. The term "reproducibility crisis" describes the problem that many empirical studies' results are impossible or at least challenging to reproduce by other researchers or the original study's authors. A replication crisis calls into question how studies are conducted, how they are presented, and the validity of results (Fanelli, 2009). Thus, an increase in replication research is desirable in many disciplines, including IS research, to strengthen existing knowledge and the theoretical base from which new studies start.

Efforts to facilitate replication research in the IS community have already been made, ranging from providing better guidelines of how and why to carry out replication research (Dennis \& Valacich, 2014) to establishing conference tracks and journals focusing on publishing replication studies (AMCIS, 2018; TRR, 2018). Nonetheless, replication research remains a rarity in IS research (46 total published replication studies in the basket of eight journals, AIS Conferences, and AIS Transactions on Replication Research up to October 2018). We would argue that this is partly caused by the common perception of replication research to be mundane and boring (Lindsay \& Ehrenberg, 1993). Replication research is perceived to be unable to provide interesting theoretical contributions, hindering replication studies from being published in well-regarded outlets (e.g., reviewers and editors are biased against publishing replication studies) (Dennis \& Valacich, 2014).

This challenge has been addressed in various ways in several disciplines. Recommendations include teaching the importance of replication and reproducibility (Frank \& Saxe, 2012), pre-registration of studies (Simons et al., 2014) and sharing studies data online (loannidis, 2016). Similarly, researchers are called to conduct replication studies in the IS community by highlighting the value of replication research (e.g., Dennis and Valacich 2014; Niederman and March 2014). However, the quest to find ways to make replication research more appealing still seems unsolved, needing further investigation. Against this background, this article aims to address the question of:

\section{How can we make replication research more appealing to conduct and publish?}

We want to address the misconception that replication studies have to be exact replications of the original and are thereby perceived as boring (Lindsay \& Ehrenberg, 1993). In order to contribute towards making replication research more interesting and appealing, we approach it by trying to learn from other disciplines, which have a more pronounced replication culture. To be specific, we analyzed the discipline of neuroscience in this paper.

The replication culture of neuroscience distinguishes itself from others because neuroscience is the interface between psychology, biology, and medicine (for a review on the emergence of neuroscience as a field of its own right, see Cowan et al. (2000)). From these disciplines, psychology has been the most affected by the so-called replication crisis (Open Science Collaboration, 2015) and has the problem of hardto-control experiment parameters and context of an experiment (Stroebe \& Strack, 2014). Neuroscience, classified as natural science, also utilizes well-defined experimental settings, which are much easier to be replicated. Neuroscience explores the biological basis of psychological hypotheses and theories. This exploration necessitates a replication of previously observed original behavioral findings to correlate them with neural recordings. Additionally, since neuroscience utilizes a plethora of different methods (e.g., imaging or electrical recordings), each with their limitations, a phenomenon cannot be fully understood with just one experiment and has to be replicated by a different group with different methodological strengths to be thoroughly investigated. Lastly, the neuroscience community acknowledges the need for replication (Kellmeyer, 2017; Steckler, 2015) and actively promotes the publishing of replication studies in all their 
journals (Bernard, 2016). In sum, we believe that IS can learn from neuroscience since it is also applying methods and theories from other disciplines (e.g., psychology, computer science, economics).

The relatability of neuroscience to IS research can also be seen in the emerging research field of NeurolS (Mamun et al., 2018; Riedl et al., 2017). To give an example of such interdisciplinary research, Dimoka et al. (2011) investigated a brain area known as the dorsolateral prefrontal cortex (DLPFC) and its association with the perceived ease of use of an online shopping website in a functional magnetitic resonance imaging (fMRI) experiment. Subsequent publications aimed to replicate those findings but utilizing different methods, such as transcranial direct current stimulation (Dumont et al., 2018) or functional near-infrared spectroscopy (Nissen et al., 2019). Both subsequent experiments failed to replicate the finding of Dimoka et al., calling the initial results into question. Nonetheless, it is possible that the original study is the one that was correct or that the two studies point to a contingency such that both are correct but under somewhat different conditions. The point of the replication is not to settle with only one being "correct." Against this background, conducting replication studies with various methods to investigate original findings is highly valuable for scientific progress.

In the remainder of this article, we will first provide a brief introduction to replication research. Second, we will present the observations and conclusions we made when analyzing replication studies from the field of neuroscience (see Appendix for details). Third, we discuss our findings to elicit implications for the IS community. Last, a brief conclusion is drawn, including a call for action.

\section{Replication Research}

Replication research aims to enable a scientific consensus on the proposed knowledge by testing the validity of previous studies (Berthon et al., 2002; Dennis \& Valacich, 2014; Schmidt, 2009). Hence, the aim is to investigate the ability to generalize and strengthen existing knowledge. Thus, replication studies contribute to theory by supporting or questioning the understanding of certain phenomena and research areas (Dennis \& Valacich, 2014; Niederman \& March, 2014).

Following Popper $(1959,1963)$, theories must be possible to falsify, and theories can only be refuted and never confirmed. Thus, the scientific progress consists of (1) proposing a theory, (2) trying to refute the theory, (3) improving or replacing theory to explain the investigated phenomena better (Salovaara \& Merikivi, 2015). In this context, replication research primarily addresses the second step of the scientific progress and tries to refute existing theories, which leads to strengthening theories that withstand refutation attempts (Figure 1 illustrates the process and the position of replication research).

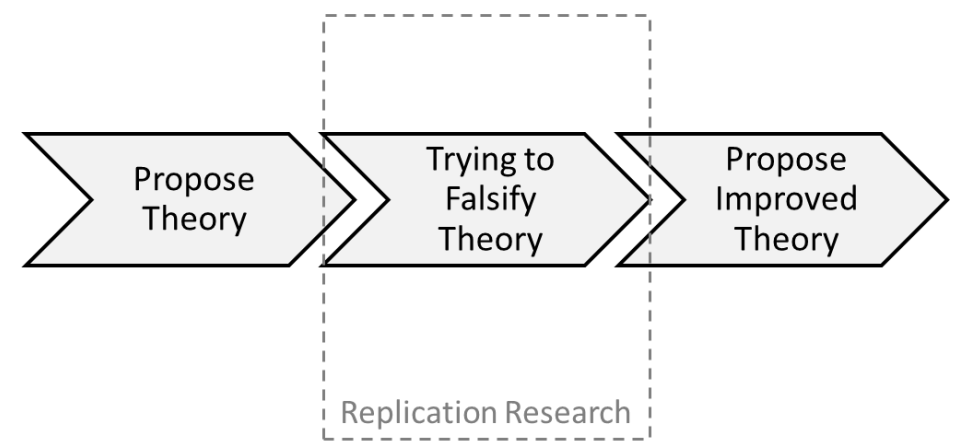

Figure 1: Process of Falsification

In this context, replication studies can address different parts of a study. To be specific, replication studies can lead to at least one of the following five outcomes (Schmidt, 2009):

(1) Finding sample errors means to search for results that were obtained based on incorrect data. The original study is replicated as closely as possible, with a new sample with the same characteristics as the original study. The original method (commonly statistical test) is repeated, i.e., the original study's $p$-value(s) by which hypotheses were supported or rejected initially. For example, in case the chance of a false-positive result is $p=0.05$ in the original study, a positive replication study (e.g., also $p=0.05$ ) leads to a lower $p$-value overall ( $p=0.05 \times 0.05=0.0025$ ), making chance results (e.g., sample error) doubtful (Schmidt, 2009). 
(2) Controlling for lack of internal validity is important to rule out external variables that interact with the study design and results (Schmidt, 2009). Overall, it is concerned with "whether the researcher provides a plausible causal argument, logical reasoning that is powerful and compelling enough to defend the research conclusion" (Gibbert et al. 2008, p.1466).

(3) Uncovering fraud addresses the problem of human interference in the reported results. Although journals and conferences require authors to comply with their codes of research conduct, fraud cases have been observed in the past (Wilmshurst, 2002). The original study is replicated as precisely as possible to identify any deviations in results, which are caused by potential human interference (e.g., fraud) (Schmidt, 2009).

(4) Expanding or generalizing results to cover a larger or different context addresses the generalization of initial results across population and time. Against this background, a replication study is conducted to investigate whether the original results are specific to a certain population or context (Schmidt, 2009). To give an example, a common discussion in the literature addresses whether university students are a valid subject group for certain research (Compeau et al., 2012). Hence, replication studies can be conducted to verify a study conducted amongst a student population by replicating it with non-student data. This increases the scope of the original study.

(5) Verifying original hypotheses means to provide hypothesis-conformation or hypothesisdisconfirmation for an existing theory by repeating previous investigations (Snyder \& White, 1981). To verify a behavioral research hypothesis means to reach beyond the function to support results by repeating the original experimental procedure. This means that a replication study follows different experimental arrangements, designed to independently reconsider the original study's hypothesis (Schmidt, 2009). Hence, the aim is to investigate the prior thinking and respond appropriately based on findings. Thus, the theoretical contribution lies in the iterative improvement and elaboration of an existing theory, supporting them with empirical results or refuting them because of contrary replication results (Compeau et al., 2012).

A variety of replication approaches and types have been advanced over the years (Berthon et al., 2002; Dennis \& Valacich, 2014; Gómez et al., 2014). The most prominent categorization in the IS research community is from Dennis and Valacich (2014):

(1) Exact replications share the same context and method with the original study. All treatments, methods, and measures are identical to the original research. Furthermore, the context remains the same, so if the original study used employees of a Chinese automotive company, the replication study would do so as well (Dennis \& Valacich, 2014).

(2) Methodological replications apply the same method as the original study but in a different context. For instance, this means that instead of employees of a Chinese automotive company, the replication study might use German undergraduate students (Dennis \& Valacich, 2014).

(3) Conceptual replications investigate the same research questions via different means or context. Thus, they seek to answer the same research question testing the same hypotheses but with different measures, treatments, contexts, or analytical methods. For example, in the replication study, the wording of items used to measure key constructs might be altered (Dennis \& Valacich, 2014).

\section{Three New Replication Study Categories}

To find ways to make replication research more appealing to conduct and publish, we looked at the discipline of neuroscience (see Appendix A for the research approach and Appendix B for a detailed summary of analysis results). When looking at studies from neuroscience, we discovered that not all studies fall into the categories provided by Dennis and Valacich (2014). Besides exact, method, and conceptual replication, we found more study categories (see Table 1 for a summary of current and additional study categories).

Transfer replications are studies that apply a similar method as the original study to test the original (or very similar) hypotheses in a different context. Such a study primarily tests the boundary of a theory - does it extend into this additional domain, or does it apply only within its previous bounds? Specifically, the goal is not to change, extend, or add to the existing theory, but rather to test or increase its external validity and transferability to other contexts. An example is the study of Blizzard et al. (2016). Their study explored lower motor neuron degeneration after spinal cord injury, which was originally explored by using the same method on the subject of the model organism "rat." In the replication study, the subject was changed to be the model organism "mice," and the method was adapted accordingly. When looking into IS literature, we found the 
study of Xu et al. (2012) to be a somewhat fitting example (Note, it does not fit perfectly and would suggest that a perfect fit has yet to be found). In this study, the authors transferred the model of Malhotra et al. (2004) regarding users' information privacy concerns to the context of smartphones. For this, they had to adjust their applied instruments.

Method Replication studies aim at validating existing methods by using a dataset that is either simulated or has a known ground truth. Often, two methods are compared to see which of them is more suited to a given task under given boundaries and requirements (e.g., efficiency or precision). By using a dataset that has a known result (e.g., correlations are present or distinctly not present - either naturally or artificially), the methods can be compared. For instance, if a method finds a correlation where none should be found, the method's validity can be called into question. Those studies are imperative for natural science research, since knowing the strengths and limitations of a method is vital in judging the original research results. A good example is Tudorascu et al. (2016), in which the authors compare two popular programs used for functional magnetic resonance imaging analysis. In the context of IS research, this potential approach to replication research seems to exist (e.g., Kim and Malhotra 2005) but lacks recognition as a replication study category so far.

We presented the previously described study categories at the Americas Conference on Information Systems 2019 conference (Greulich \& Brendel, 2019), which were well received. Nonetheless, when discussing the completeness of the new set of replication categories, an additional category was proposed by the plenum:

Comparison replication studies try to compare theories to validate their claims to explain a given context adequately. Specifically, there can be competing theories, explaining a similar phenomenon based on a different set of assumptions. For instance, the technology acceptance model (TAM) (Davis, 1989) and the unified theory of acceptance and use of technology (UTAUT) (Venkatesh et al., 2003) both address technology acceptance via a different conceptualization and level of complexity (van Raaij \& Schepers, 2008). Through a comparison replication study, researchers could be provided with evidence for selecting one or the other theory to explore a new context. Similar to method replication, studies falling into this category can also be found in IS research (e.g., Trang et al. 2014) but are not considered part of the replication research spectrum.

Lastly, we would like to acknowledge that transfer replications can be categorized as a sub-category of conceptual replications (Dennis \& Valacich, 2014). The category of conceptual replications includes all studies that alter the applied method. Furthermore, the context can be changed, but such a change is not necessarily considered a conceptual replication. Based on this consideration, a second sub-category can be distinguished besides transfer replications:

Context Replication studies apply a different method to test the original (or a very similar) hypotheses in the same context. Such a replication aims to investigate whether the original theory or results are valid or a product of the applied method. Hence, a context replication study addresses the method validity and potential biases of the original research team during the selection and application of the method. An example would be the study of Mitchell and Zheng (2019), in which the original method of an experiment in a lab environment was replaced with an experiment in a classroom environment, which necessitated some changes to the original procedures. 


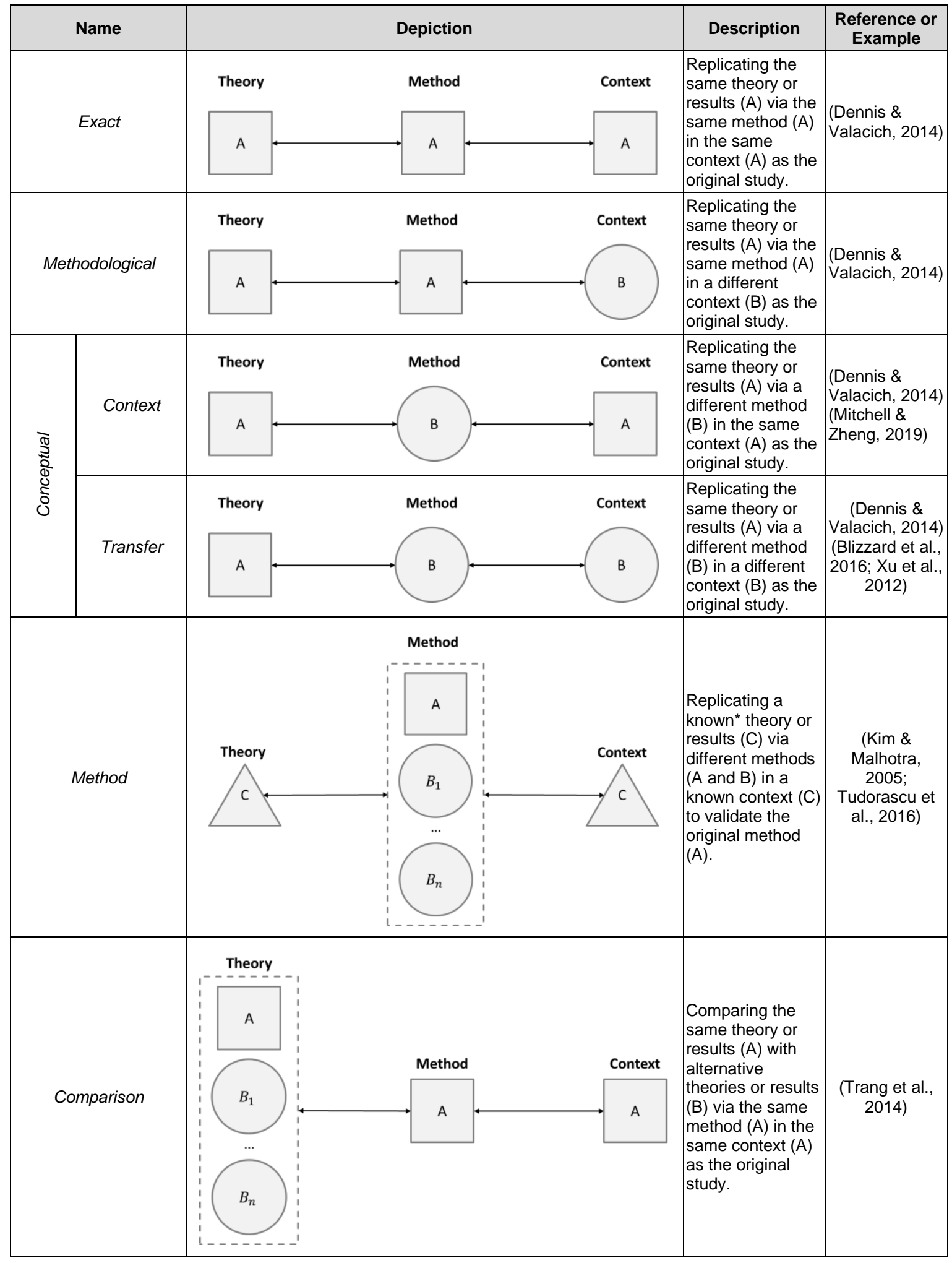

Legend: $\mathrm{A}$ (rectangle) = same as original study; $\mathrm{B}(\mathrm{circle})=$ different from original study; $\mathrm{C}$ (triangle) $=$ known and different to original study

*Note "known" means that the relation of theory and context has been well supported via various means.

Table 1: Replication Study Categories Comparison 


\section{$4 \quad$ Discussion}

This paper aimed to contribute to making replication research more appealing. In this context, we elicited replication study categories from the field of neuroscience and compared them with the common replication study categories from the IS field. Through the comparison, it appeared that the study categories transfer, context, method, and comparison replication are currently not recognized (see Table 1). In an effort to broaden the range of approaches available to scholars for replication research, we offer additional categories within which such research can be designed and executed. They provide transparency regarding the specific contributions that replication research can offer to the IS community. By defining them, we believe that researchers can better position and justify their replication research.

Firstly, a transfer replication study transfers existing theories in a new context, investigating existing theories' capabilities and limitations. Thereby, transfer replication studies can provide a foundation of subsequent studies, addressing how existing theories should be adapted to overcome limitations and eventually helping to understand new phenomena. Hence, conducting a transfer replication study can take some pressure off these studies by already transferring existing theories and highlighting areas for adaption. In the context of IS research, which is characterized by rapid changes caused by technology (Easley et al., 2000; Niederman \& March, 2014), it is crucial to reevaluate and adapt theories to stay relevant and accurate iteratively. This also includes evaluating whether the scope of theories is decreasing or expanding. Hence, before developing entirely new theories, conducting transfer replication studies can reveal where existing theories remain relevant in IS research. Furthermore, this approach would greatly fit within the scientific process proposed by Popper (1963). Before proposing a new theory, replicating, and possibly refuting existing theories can be an excellent means to sharpen the outlines of research gaps and the eventual contribution of a study. In sum, we see great potential in conducting transfer replication studies in the IS community.

Secondly, a method replication study seeks to verify a method by either comparing it to other established methods, applying it to a dataset with known ground truth (e.g., from a simulation), or both. This is different from the methodological replication proposed by Dennis and Valacich (2014), where the original study is replicated with the same method but in a different context. However, this does not question the ability of the method to get the correct results. Since the different contexts could cause differences in the outcome, and if the method is itself flawed, it might lead to erroneous replications. Hence, we see the method replication study category as an integral part of proper scientific work, which can help rule out flawed methodology as a cause for failed replications.

Thirdly, comparison replication studies promise to be a valuable means to direct researchers in identifying theories for extension or even developing new theories. These replication studies do not only replicate the addressed theories individually but try to compare them in order to elicit differences and sharpen limitations. This comparison process is especially important because often various theories can be applied to explain similar behavior. For instance, motivation can be explained following various theories, ranging from the selfdetermination theory over goal orientation theory to attribution theory (Cook \& Artino, 2016). Based on the results of a comparison replication study, future research can better articulate and justify selecting a specific theory for a particular context.

\section{Conclusion}

We are not the first (e.g., Dennis and Valacich 2014; Niederman and March 2014; Salovaara and Merikivi 2015) and hopefully not the last to call for more replication research in the IS research community, making an effort to change our perception regarding the value of replication research. In order to provide a more interesting replication study landscape, we propose three additional replication study categories: (1) transfer, (2) method, and (3) comparison.

In this context, comparing each replication study category's scope should spark the question: what constitutes a replication study and differentiates it from "regular" research? Specifically, the replication study category of transfer replication triggers this question. "Borrowing" theories by transferring them from other disciplines or topics to explain other phenomena is a standard procedure and necessary to appropriately approach the phenomena to be investigated (Moeini et al., 2019, 2020). One example in this context is the reapplication of the technology acceptance model, which has been proven to provide a foundation to approach technology acceptance in various contexts (Salovaara \& Merikivi, 2015).

Thus, there needs to be a differentiation between reusing and replication of a theory. Based on our sample of neuroscience replication studies and our understanding of replication research, we argue that the 
differentiating factor is the research question and goal of the researchers conducting the study. A purist view on replication dictates that replication research should be conducted as closely as possible to the original study because deviations can lead to multiple results derived from ambiguous sources. In contrast, we advocate the more liberal approach to understanding replication research. We see the study's orientation as decisive. If a study is oriented towards existing knowledge (i.e., trying to verify and validate results and theories that already exist), it can be considered a replication study. Contrarily, a study that is oriented towards new knowledge (i.e., actively trying to extend an existing theory, develop new theory, and explain new phenomena) has to be classified as "regular" research. In that regard, a transfer replication study has a clear focus on existing theory. Nonetheless, a transfer study showing the limits of existing theories is a great reason to ask why the theory could not explain the phenomena. Due to this, we see a transfer study as a link between pure replication research and "regular" research. Overall, it extends the replication goal of expanding or generalizing results to cover a broader or different context (Schmidt 2009) by actively seeking contexts that are not very similar to the original.

Overall, we would like to call on the IS community to conduct and value replication studies so that in addition to creating or borrowing theory, we are engaged in constant improvement. Thus, we would increase our body of theory and have higher levels of confidence where they are supported and opportunities to investigate nuanced variations in the environment, which translates to higher knowledge in applying theory. We call on the IS research community to embrace this element of creating a more reliable, more robust, and more useful body of knowledge.

\section{Acknowledgments}

We would like to acknowledge that the first and second author contributed equally during this research project. Hence, they share the main authorship and the order was decided by coin toss. 


\section{References}

AMCIS. (2018). Replication Track at AMCIS 2018. Retrieved from: https://amcis2018.aisconferences.org/submissions/track-descriptions.

Baker, M. (2016). 1,500 scientists lift the lid on reproducibility. Nature, 533(7604), 452-454.

Bernard, C. (2016). Editorial : A message from the editor-in-chief. ENeuro, 3(1).

Berthon, P., Pitt, L., Ewing, M., \& Carr, C. L. (2002). Potential research space in MIS: A framework for envisioning and evaluating research replication, extension, and generation. Information Systems Research, 13(4), 416-427.

Blizzard, C. A., Lee, K. M., \& Dickson, T. C. (2016). Inducing chronic excitotoxicity in the mouse spinal cord to investigate lower motor neuron degeneration. Frontiers in Neuroscience, 10(March), 1-8.

Compeau, D., Marcolin, B., Kelley, H., \& Higgins, C. (2012). Research commentary-Generalizability of information systems research using student subjects-A reflection on our practices and recommendations for future research. Information Systems Research, 23(4), 1093-1109.

Cook, D. A., \& Artino, A. R. (2016). Motivation to learn: An overview of contemporary theories. Medical Education, 50, 997-1014.

Cowan, W. M., Harter, D. H., \& Kandel, E. R. (2000). The emergence of modern neuroscience: Some implications for neurology and psychiatry. Annual Review of Neuroscience, 23, 343-391.

Davis, F. D. (1989). Perceived usefulness, perceived ease of use, and user acceptance of information technology. Management Information Systems Quarterly, 13(3), 319.

Dennis, A. R., \& Valacich, J. S. (2014). A replication manifesto. AIS Transactions on Replication Research, 1, Paper 1, 1-5.

Dimoka, A., Pavlou, P. A., \& Davis, F. D. (2011). Research commentary-NeurolS: The potential of cognitive neuroscience for information systems research. Information Systems Research. Information Systems Research, 22(4), 687-702.

Dumont, L., Larochelle-Brunet, F., Theoret, H., Riedl, R., Senecal, S., \& Leger, P. M. (2018). Non-invasive brain stimulation in information systems research: A proof-of-concept study. PloS One, 13(7), e0201128.

Easley, R. W., Madden, C. S., \& Dunn, M. G. (2000). Conducting marketing science: The role of replication in the research process. Journal of Business Research, 48(1), 83-92.

Fanelli, D. (2009). How many scientists fabricate and falsify research? A systematic review and metaanalysis of survey data. PLOS One, 4(5), e5738.

Frank, M. C., \& Saxe, R. (2012). Teaching replication. Perspectives on Psychological Science, 7(6), 600604.

Gibbert, M., Ruigrok, W., \& B., W. (2008). What passes as a rigorous case study? Strategic Management Journal, 29, 1465-1474.

Gómez, O. S., Juristo, N., \& Vegas, S. (2010). Replications types in experimental disciplines. Proceedings of the 2010 ACM-IEEE International Symposium on Empirical Software Engineering and Measurement - ESEM '10.

Gómez, O. S., Juristo, N., \& Vegas, S. (2014). Understanding replication of experiments in software engineering: A classification. Information and Software Technology, 56(8), 1033-1048.

Greulich, R. S., \& Brendel, A. B. (2019). Towards a greater appeal of replication research by learning from the field of neuroscience. Paper presented at the Americas Conference on Inforamtion Systems (AMCIS), 1-10.

Hart, D. N., \& Gregor, S. (2012). Information Systems Foundations: Theory Building in Information Systems. In D. N. Hart \& S. Gregor (Eds.), Information Systems Foundations: Theory Building in Information Systems. ANU E Press.

Ioannidis, J. P. A. (2016). Anticipating consequences of sharing raw data and code and of awarding badges 
for sharing. Journal of Clinical Epidemiology, 70, 258-260.

Kellmeyer, P. (2017). Ethical and legal implications of the methodological crisis in neuroimaging. Cambridge Quarterly of Healthcare Ethics, 26, 530-554.

Kim, S. S., \& Malhotra, N. K. (2005). Predicting system usage from intention and past use: Scale issues in the predictors. Decision Sciences, 36(1), 187-196.

King, G. (1995). Replication, replication. Political Science and Politics, 28(3), 541-559.

Lindsay, R. M., \& Ehrenberg, A. S. C. (1993). Commentaries the design of replicated studies. American Statistician, 47(3), 217-228.

Malhotra, N. K., Kim, S. S., \& Agarwal, J. (2004). Internet users ' information privacy concerns ( IUIPC ): The construct, the scale, and a causal model. Information Systems Research, 15(4), 336-355.

Mamun, R. Al, David, A., Mai, B., Kim, D. J., \& Parsons, T. D. (2018). The first 10 years of NeurolS : A systematic literature review of NeurolS. Paper presented at the Hawaii International Conference on System Sciences, 9, 226-234.

Mitchell, A., \& Zheng, L. (2019). Examining longhand vs. laptop debate: A replication study. AIS Transactions on Replication Research, 5, Paper 9, 1-15.

Moeini, M., Boyka, S., Galliers, R., \& Wilson, A. (2020). Theory borrowing in IT-rich contexts: Lessons from IS strategy research. Journal of Information Technology, 35(3), 270-82.

Moeini, M., Rahrovani, Y., \& Chan, Y. E. (2019). A review of the practical relevance of IS strategy scholarly research. Journal of Strategic Information Systems, 28(2), 196-217.

Niederman, F., \& March, S. (2014). Reflections on Replications. AIS Transactions on Replication Research, 1, Paper 7, 1-16.

Nissen, A., Krampe, C., Kenning, P., \& Schütte, R. (2019). Utilizing mobile fNIRS to investigate neural correlates of the TAM in eCommerce. Paper presented at the International Conference on Information Systems (ICIS).

Open Science Collaboration. (2015). Estimating the reproducibility of psychological science. Science, 349(6251).

Popper, Karl Raimund. (1963). Conjectures and refutations: The growth of scientific knowledge. Routledge and Kegan Paul.

Popper, Karl Raimund. (1959). The Logic of Scientific Discovery. Hutchinson \& Co.

Riedl, R., Fischer, T., \& Léger, P. M. (2017). A decade of NeurolS Research: Status quo, challenges, and future directions. Paper presented at the International Conference on Information Systems (ICIS).

Salovaara, A., \& Merikivi, J. (2015). IS research progress would benefit from increased falsification of existing theories. Paper presented at the European Conference on Information Systems (ECIS), Paper 157.

Schmidt, S. (2009). Shall we really do it again? The powerful concept of replication is neglected in the social sciences. Review of General Psychology, 13(2), 90-100.

Simons, D.J., Holcombe, A.O., \& Spellman, B.A. (2014). An introduction to registered replication reports at perspectives on psychological science. Perspectives on Psychological Science, 9(5), 552-555.

Snyder, M., \& White, P. (1981). Testing hypotheses about other people strategies of verification and falsification. Personality and Social Psychology Bulletin, 7(1), 39-43.

Steckler, T. (2015). Editorial: Preclinical data reproducibility for R\&D - The challenge for neuroscience. Springer Plus, 4(1).

Stroebe, W., \& Strack, F. (2014). The alleged crisis and the illusion of exact replication. Perspectives on Psychological Science, 91(1), 59-71.

Trang, S., Zander, S., \& Kolbe, L. M. (2014). E-business adoption at the firm level : Comparing the predictive power of competing IS adoption models. Paper presented at the International Conference on Information Systems (ICIS), 1-20. 
TRR. (2018). AIS Transactions on Replication Research. https://aisel.aisnet.org/trr/.

Tudorascu, D. L., Karim, H. T., Maronge, J. M., Alhilali, L., Fakhran, S., Aizenstein, H. J., Muschelli, J., Crainiceanu, C. M., \& Brett, M. (2016). Reproducibility and bias in healthy brain segmentation: Comparison of two popular neuroimaging platforms. Frontiers in Neuroscience, 10, Paper 503, 1-8.

van Raaij, E. M., \& Schepers, J. J. L. (2008). The acceptance and use of a virtual learning environment in China. Computers \& Education, 50(3), 838-852.

Venkatesh, Morris, Davis, \& Davis. (2003). User acceptance of information technology: Toward a unified view. MIS Quarterly, 27(3), 425-478.

Wilmshurst, P. (2002). Fraud in research. Clinical Medicine, 2(2), 159-160.

Xu, H., Gupta, S., Rosson, M. B., \& Carroll, J. M. (2012). Measuring mobile users' concerns for information privacy. Paper presented at the International Conference on Information Systems, 1-16. 


\section{Appendix A: Literature Review Approach}

We base our research approach on the literature review approach, specifically on the seminal works of Webster and Watson (2002) and Vom Brocke et al. (2009). Following the taxonomy of literature reviews of Cooper (1988), we designed our research approach to conduct a literature review of the following characteristics:

Focus - Practices and Applications: Our literature review focuses on research practices and applications in replication research.

Goals - Integration: Our literature review aims at integrating replication research practices from the field of neuroscience within the IS research domain. Hence, the aim is not to critique. We want to look at how to make replication research more approachable and appealing - and there is nobody at fault for the lack of attractiveness.

Perspective - Neutral Representation: The literature review is supposed to provide a neutral analysis of categories of conducted replication studies and what we can learn from them.

Coverage - Representative: The selected literature is supposed to be a presentative sample of replication research in neuroscience.

Organization - Conceptual: A conceptual perspective is required to identify categories of replication studies and therefore applied in our literature review.

Audience - General Scholars: The literature review is presumed to provide insights into replication research practices that are interesting for the entire IS community.

The research approach has three phases to enable the conduction of the previously described literature review:

Phase 1-Review: How is replication research carried out in neuroscience? In this phase, replication studies from the field of neuroscience were reviewed to identify replication study categories and practices in neuroscience.

Phase 2 - Comparison: How does replication research in neuroscience differentiate from replication research in the IS community? In the second phase, seminal works on replication research are reviewed and matched with the first phase's results to achieve a coherent and complete picture of the status quo.

Phase 3 - Interpretation: What are the implications for the IS community? In the last phase, the similarities and differences of replication studies are interpreted to elicit implications on making replication research more appealing for the IS community. This is also the phase where we asked other scholars to review our conclusions and allowing us to reflect on and refine our interpretations.

The individual phases' steps and relations are illustrated in Figure A1 and are described in the following sub-sections.

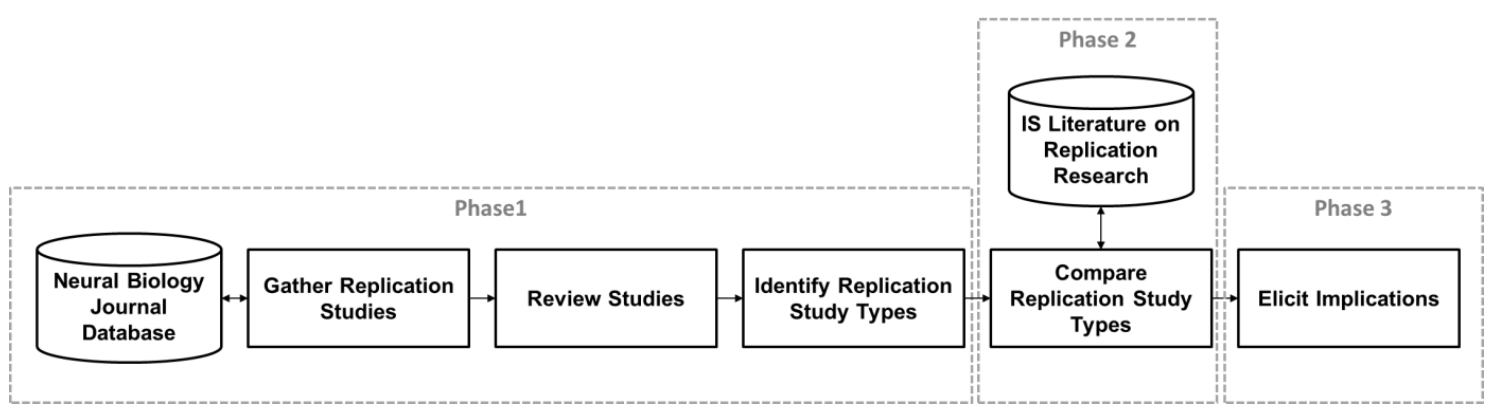

Figure A1: Research Process 


\section{Phase 1: Review of Replication Studies in Neuroscience}

In the first phase, two points guided us to select the field of neuroscience as a reference point for finding aspects to make replication research more appealing. Firstly, as discussed in the introduction, neuroscience has an influential replication culture. Secondly, one of the authors is a researcher in the field of neuroscience. This is especially beneficial, as understanding the field and methods used is essential to judge a paper's content.

Based on the author's experience in neuroscience, we decided to select an open-access neuroscience journal for our literature search. This decision was made because replication studies are often considered not to be innovative enough for the traditional journals (showcasing that even the neurosciences are still suffering some shortcomings in replication research). Thus, the neuroscience community is actively promoting open access to combat this publication bias (Koch and Jones 2016). According to the 2016 Journal Citation Reports (Journal Citation Reports 2016), the most cited neuroscience open access journals are first "Frontiers in Human Neuroscience," second "Frontiers in Cellular Neuroscience," and third "Frontiers in Neuroscience." To ensure the review of a representative cross-section of the field, we picked the "Frontiers in Neuroscience" journal. However, this had the unfortunate side effect that it was impossible to use a keyword search to get replication studies.

The literature search was conducted in 2017, and we decided to include all publications of 2016 in our search (each year has a volume of around 722 publications), providing the manageable number of 631 publications for our filtering process. During the search, a two-step approach was applied. Firstly, the author, with a background in neuroscience, identified studies via title and abstract. The articles were filtered by two of the authors, one with a Ph.D. in IS and one doctoral candidate in neuroscience. We reviewed the titles and abstracts of this selection for replication studies. Studies that presented novel results were excluded, as were review articles. We exclusively included articles that specifically replicated previous research. Eventually, the sample was reduced to 59 articles. Those articles were then reviewed in depth over a full year, leading to the exclusion of another five articles.

\section{Phase 2: Comparison of Replication Study Categories from Neuroscience and Information Systems Research}

Based on the results of the first phase, we compared the elicited replication study categories with the replication study categories of IS research. In this context, we decided to follow the replication study categories used in the AIS Transactions on Replication Research journal (Dennis and Valacich 2014; TRR 2018). In that regard, both authors revised the previously made categorizations and compared them to the categories found in the IS community.

\section{Phase 3: Interpretation of Differences and Elicitation of Implications}

In the last phase, we interpreted the differences in replication categories by reflecting on what the differences can mean for future replication research. To be specific, we compared the falsification process by Popper (1963) and which roles the newly developed replication categories can play within it. 


\section{Appendix B: Literature Analysis}

While going through our literature set, we found five different categories of replication studies (see Table B1), which we will describe in the following.

Studies in the first category retest the same hypothesis as the original study, using the same method, which can be called exact replication (Dennis and Valacich 2014), as the original research is repeated as closely as possible. For example, the study of Morrison et al. (2016) investigates the reliability of fMRI brain mapping before implantation of direct brain stimulation devices (DCS). Here, several studies already exist but are often marred by small sample size as the number of available patients per brain surgery center is limited. Hence, the study adds to an existing body of work with the same methods to better evaluate the previously reported effects. In total, we found three studies of this kind.

The second category retests the same hypothesis as the original work, sometimes with an extension, but follows a different method. Hence, these studies can be called context replications. Such studies aim to ensure that the previous results are not caused by misuse or unsuitability of the original method. For example, Coutlee et al. (2016) are retesting the involvement of the intraparietal sulcus (ISP) in risk evaluation, which was shown by fMRI. The authors expose subjects to the same risky-choice task as the original study, but after the IPS was disabled with transcranial magnetic stimulation. In total, we found ten studies for this category.

\begin{tabular}{|l|l|l|l|c|l|}
\hline \multicolumn{1}{|c|}{ Name } & \multicolumn{1}{|c|}{ Hypothesis } & \multicolumn{1}{c|}{ Method } & \multicolumn{1}{c|}{ Model } & Example \\
\hline Exact & Identical & Identical & Identical & 10 & Coutlee et al. (2016) \\
\hline Conceptual & $\begin{array}{l}\text { Identical } \\
\text { (sometimes } \\
\text { extended })\end{array}$ & Different & Identical & 11 & Blizzard et al. (2016) \\
\hline Methodological & Identical & Identical & Different & 5 & Raffa et al. (2016) \\
\hline Transfer & Identical & Different & Different & Tudorascu et al. (2016) \\
\hline Method & Identical & $\begin{array}{l}\text { Comparing different } \\
\text { methods for the same } \\
\text { outcome or against } \\
\text { ground truth }\end{array}$ & $\begin{array}{l}\text { Identical or simulated } \\
\text { with known ground } \\
\text { truth }\end{array}$ & 25 & Thon et \\
\hline
\end{tabular}

Table B1: Elicited Replication Research Categories

In our third category, studies investigate a similar context compared to the original study and apply the same methodological approach. In this context, similar means that the replication study authors try to find the same results as the original study, but in a different context. Hence, this study category can be called methodological replication. An example is the study of Blizzard et al. (2016). In their study, they explored lower motor neuron degeneration after spinal cord injury, which was originally explored by using the same method on the subject of the model organism "rat." In the replication study, the subject was changed to be the model organism "mice." Overall, we found 11 studies for this category.

We called the fourth category transfer replication. Transfer studies use the same method but to test a different yet similar hypothesis, emphasizing that the hypothesis is similar to the original one. For example, Raffa et al. (2016) uses a well-established method for preoperative trace motor circuitry in brain cancer patients and attempts to trace the language circuitry. This firstly retests the used method and secondly, the underlying assumption that both hypotheses are similar enough. In total, we found five studies for this category.

Lastly, some studies have the aim of method replication. This is often done using a dataset that is either simulated or has a known ground truth and is then explored with the method. Often, two methods are compared to see which of them is more suited to a given task. Those studies are imperative for natural science research, since knowing the strengths and limitations of a method is vital in judging the results of original research. A good example is Tudorascu et al. (2016), in which the authors compare two popular programs used for $\mathrm{fMRI}$ analysis. We found 25 examples. 


\section{About the Authors}

Alfred Benedikt Brendel was an assistant professor ("akademischer Rat") at the University of Goettingen, Germany and has been appointed professor at the Technische Universität Dresden in October of 2020 and holds the chair for business informatics, esp. intelligent systems and services. Alfred received his Doctor's degree in management science, specializing in Business Information Systems, from the University of Goettingen. His research focuses on the application of Design Science Research to develop novel and innovative design principles and theories. His main areas of research are digital health, smart mobility, and persuasive system design. His research has been published in the proceedings of leading conferences, such as the International Conference on Information Systems and European Conference on Information Systems, and journals, such as Business \& Information Systems Engineering and Information Systems Frontiers.

R. Stefan Greulich is a PhD student at the German Primate Center (Deutsches Primatenzentrum GmbH) in Göttingen. He holds a master's degree in biophysics and is currently researching motor neuroscience in humans and macaques. He specializes in applying machine learning algorithms to function magnetic resonance imaging for high order neuro processes such as language switching or motor initiation. His previous work has stretched from optogenetics to automated analysis of confocal microscopy recordings. He has co-authored papers in Neuroimage, Brain and Language and PLOS Biology.

Dr. Fred Niederman serves as Shaughnessy Endowed Professor at Saint Louis University. His PhD is from the University of Minnesota in 1990. He has recently begun serving as editor in chief for Communications of Association for Information Systems. He served as a Department Editor for ICT for Project Management Journal (2014-2020). He has published peer reviewed studies in Basket of eight journals, including Management Information Systems Quarterly, Journal of Association for Information Systems, Journal of Strategic Information Systems, and Journal of Management Information Systems. He serves on the editorial boards for the DATABASE for Advances in Management Information Systems, and Journal of International Management. He has edited or co-edited numerous special issues on a wide variety of topics including agile development and project management for Project Management Journal and "Breakthrough Ideas" for Communications of Association for Information Systems. His areas of research interest include: IS personnel, IS project management, philosophy of science applied to IS, qualitative IS research methods, effects on IS of mergers and acquisitions, global IS, and group collaboration and teams. He is proud to be counted as a member of the "circle of compadres" for the KMPG PhD Project.

Simon Trang is an Assistant Professor and holds the Chair of Information Security and Compliance at the Department of Business Administration, University of Goettingen. He received his Ph.D. in management science, specializing in management information systems, from the University of Goettingen. His work focuses on information security management, strategic IT management, and sustainable IS. His research has been published in outlets, including the Journal of the Association for Information Systems, European Journal of Information Systems, Information Systems Frontiers, and a number of refereed conference proceedings, such as International Conference on Information Systems and European Conference on Information Systems.

Copyright $(02020$ by the Association for Information Systems. Permission to make digital or hard copies of all or part of this work for personal or classroom use is granted without fee provided that copies are not made or distributed for profit or commercial advantage and that copies bear this notice and full citation on the first page. Copyright for components of this work owned by others than the Association for Information Systems must be honored. Abstracting with credit is permitted. To copy otherwise, to republish, to post on servers, or to redistribute to lists requires prior specific permission and/or fee. Request permission to publish from: AIS Administrative Office, P.O. Box 2712 Atlanta, GA, 30301-2712 Attn: Reprints or via e-mail from ais@aisnet.org. 


\section{B. Mass univariate analysis of project III}

\section{B.1. Materials \& Methods}

For the mass univariate analysis, the EPI volumes underwent spatial realignment and correction for slice time acquisition. For the spatial realignment, we again used the realignment produced by the Siemens scanner, the correction for slice time acquisition was performed with SPM 12, as was all following analysis steps. The corrected volumes were normalized to the $\mathrm{MNI}$ template, resliced to $2 \times 2 \times 2$ resolution and smoothed with a Gaussian smoothing kernel (FWHM $8 \mathrm{~mm}$ isotrop).

In the GLM model, the onset of the go-cue was convolved with the HRF for each combination of conditions as a single regresser. The MT was removed as a regresser of non interest in an identical way as in the MVPA analysis, as were the movement parameters of the subject. Population statistic was implemented by a mixed effects model. In addition to the effects described in the MVPA analysis, we also considered the mean effect over all conditions. Correction for multiple testing was by family wise error correction (FWE). The significance threshold was set to $\alpha=0.05[\mathrm{FWE}$ at the voxel level. 


\section{B.2. Results}

\section{B.2.1. Mean effect}

See figure B.1 and table B.1 on this page and on page 181

Table B.1.: Mean effect. Location of the lowest p-value (voxel wise FWE corrected) and size of surrounding cluster in voxels. The clusters are sorted by ascending order for the peak p-value. The labels are sorted by proportion within the cluster, with the label of the peak location in italics.

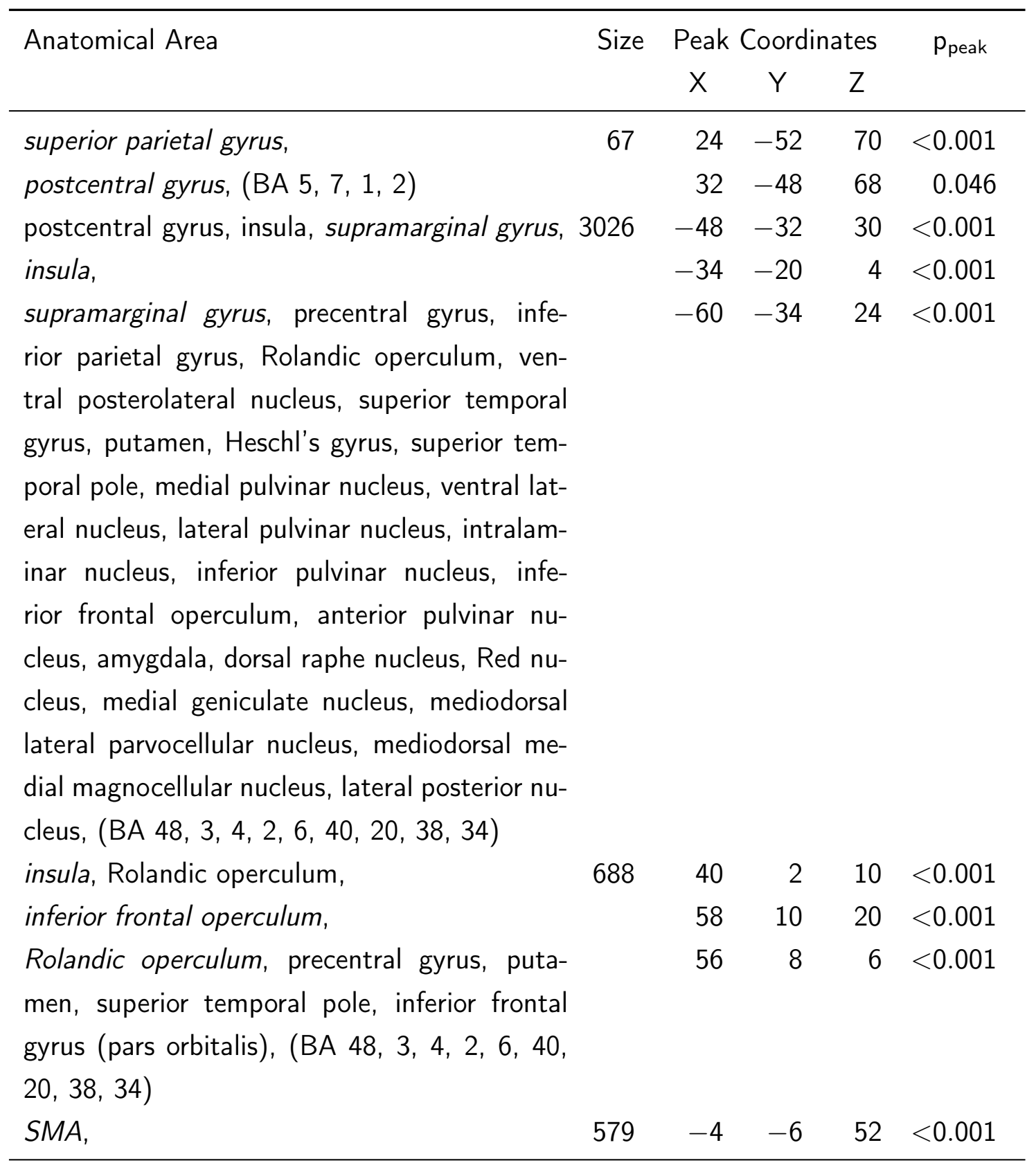

Continues on next page 
Table B.1.: Mean effect continued.

\begin{tabular}{|c|c|c|c|c|c|}
\hline \multirow[t]{2}{*}{ Anatomical Area } & \multirow[t]{2}{*}{ Size } & \multicolumn{3}{|c|}{ Peak Coordinates } & \multirow[t]{2}{*}{$\mathrm{p}_{\text {peak }}$} \\
\hline & & $x$ & Y & Z & \\
\hline$S M A$ & & 8 & -4 & 60 & $<0.001$ \\
\hline $\begin{array}{l}\text { superior frontal gyrus, middle cingulate gyrus, } \\
\text { precentral gyrus, paracentral lobue, middle cin- } \\
\text { gulate gyrus, (BA } 6,4,24)\end{array}$ & & 26 & -12 & 56 & $<0.001$ \\
\hline supramarginal gyrus, postcentral gyrus, & 582 & 56 & -26 & 32 & $<0.001$ \\
\hline Rolandic operculum, inferior parietal gyrus & & 54 & -20 & 22 & $<0.001$ \\
\hline postcentral gyrus, (BA 48, 2, 40, 3, 43, 1) & & 60 & -18 & 34 & 0.001 \\
\hline superior parietal gyrus, $(\mathrm{BA} 7,5)$ & 34 & -30 & -48 & 72 & $<0.001$ \\
\hline $\begin{array}{l}\text { precentral gyrus, inferior frontal operculum, } \\
\text { (BA } 6,44,4)\end{array}$ & 94 & -56 & 6 & 28 & $<0.001$ \\
\hline postcentral gyrus, & 137 & 44 & -30 & 58 & 0.001 \\
\hline postcentral gyrus, & & 52 & -24 & 54 & 0.004 \\
\hline $\begin{array}{l}\text { superior parietal gyrus, inferior parietal gyrus, } \\
(\mathrm{BA} 40,2,1,3)\end{array}$ & & 40 & -44 & 58 & 0.016 \\
\hline $\begin{array}{l}\text { lingual gyrus, cerebellum (IV, V), vermis (IV, } \\
\mathrm{V}),(\mathrm{BA} 3,240,1)\end{array}$ & 85 & 10 & -58 & -8 & 0.002 \\
\hline middle cingulate gyrus, $\mathrm{SMA}(\mathrm{BA} 24,32)$ & 64 & 2 & 8 & 42 & 0.005 \\
\hline SMA & 143 & -12 & -8 & 74 & 0.005 \\
\hline $\begin{array}{l}\text { SMA, superior frontal gyrus, precentral gyrus, } \\
\text { paracentral lobule, }(\mathrm{BA} 6)\end{array}$ & & -12 & -10 & 62 & 0.023 \\
\hline ventral posterolateral nucleus, & 143 & 18 & -20 & 16 & 0.006 \\
\hline $\begin{array}{l}\text { ventral posterolateral nucleus, ventral lateral } \\
\text { nucleus, pulvinar inferior nucleus, pulvinar lat- } \\
\text { eral nucleus, }\end{array}$ & & 18 & -24 & 6 & 0.008 \\
\hline $\begin{array}{l}\text { caudate nucleus, pulvinar medial nucleus, lat- } \\
\text { eral posterior nucleus, medial geniculate nu- } \\
\text { cleus, intralaminar nucleus, ventral anterior nu- } \\
\text { cleus }\end{array}$ & & 18 & -8 & 20 & 0.022 \\
\hline superior frontal gyrus, precentral gyrus, (BA 6) & 24 & -24 & -8 & 62 & 0.012 \\
\hline $\begin{array}{l}\text { Pallidum, ventral anterior nucleus, ventral lat- } \\
\text { eral nucleus }\end{array}$ & 16 & -14 & -4 & 2 & 0.013 \\
\hline
\end{tabular}

Continues on next page 
Table B.1.: Mean effect continued.

\begin{tabular}{|c|c|c|c|c|c|}
\hline \multirow[t]{2}{*}{ Anatomical Area } & \multirow[t]{2}{*}{ Size } & \multicolumn{3}{|c|}{ Peak Coordinates } & \multirow[t]{2}{*}{$p_{\text {peak }}$} \\
\hline & & $X$ & Y & Z & \\
\hline lingual gyrus, calcarine gyrus, $(\mathrm{BA} 17,18)$ & 35 & 0 & -80 & -4 & 0.017 \\
\hline lingual gyrs, (BA 18) & 5 & -12 & -68 & -6 & 0.02 \\
\hline SMA $(\mathrm{BA} 6)$ & 13 & 2 & -6 & 74 & 0.022 \\
\hline middle frontal gyrus, (BA 45) & 4 & 46 & 36 & 32 & 0.024 \\
\hline precentral gyrus, (BA 1) & 1 & -20 & -18 & 82 & 0.025 \\
\hline middle frontal gyrus, $(\mathrm{BA} 46,45)$ & 3 & 40 & 36 & 36 & 0.027 \\
\hline superior ACC (BA 24) & 9 & 6 & 18 & 26 & 0.027 \\
\hline $\begin{array}{l}\text { postcentral gyrus, supramarginal gyrus, } \mathrm{BA} \\
43,48,1,3)\end{array}$ & 10 & -60 & -20 & 38 & 0.028 \\
\hline lingual gyrus, (BA 18, 17) & 12 & -6 & -66 & 4 & 0.028 \\
\hline insula, (BA 48) & 6 & 36 & -16 & 4 & 0.03 \\
\hline SMA (BA 6) & 2 & 10 & 8 & 66 & 0.032 \\
\hline middle cingulate gyrus, (BA 23) & 3 & 12 & -24 & 38 & 0.035 \\
\hline middle frontal gyrus, $(\mathrm{BA} 46,45)$ & 3 & 42 & 40 & 26 & 0.035 \\
\hline $\begin{array}{l}\text { superior parietal gyrus, postcentral gyrus, BA } \\
2,5)\end{array}$ & 3 & -22 & -44 & 64 & 0.036 \\
\hline insula, (BA 48) & 5 & 36 & 18 & 8 & 0.037 \\
\hline caudate nucleus & 1 & 10 & 12 & 14 & 0.039 \\
\hline ventral anterior nucleus & 2 & 14 & -2 & 2 & 0.04 \\
\hline precentral gyrus, (BA 6) & 1 & -22 & -16 & 80 & 0.045 \\
\hline
\end{tabular}

\section{Main effect grasp orientation}

This contrast showed no significant voxels.

\section{Main effect grasp type}

This contrast showed no significant voxels.

\section{Reduced main effect predictability}

See figure B.2 and table B.2 on page 182 and on page 183 
$-\log \left(\mathbf{p}_{\text {uncor. }}\right)$

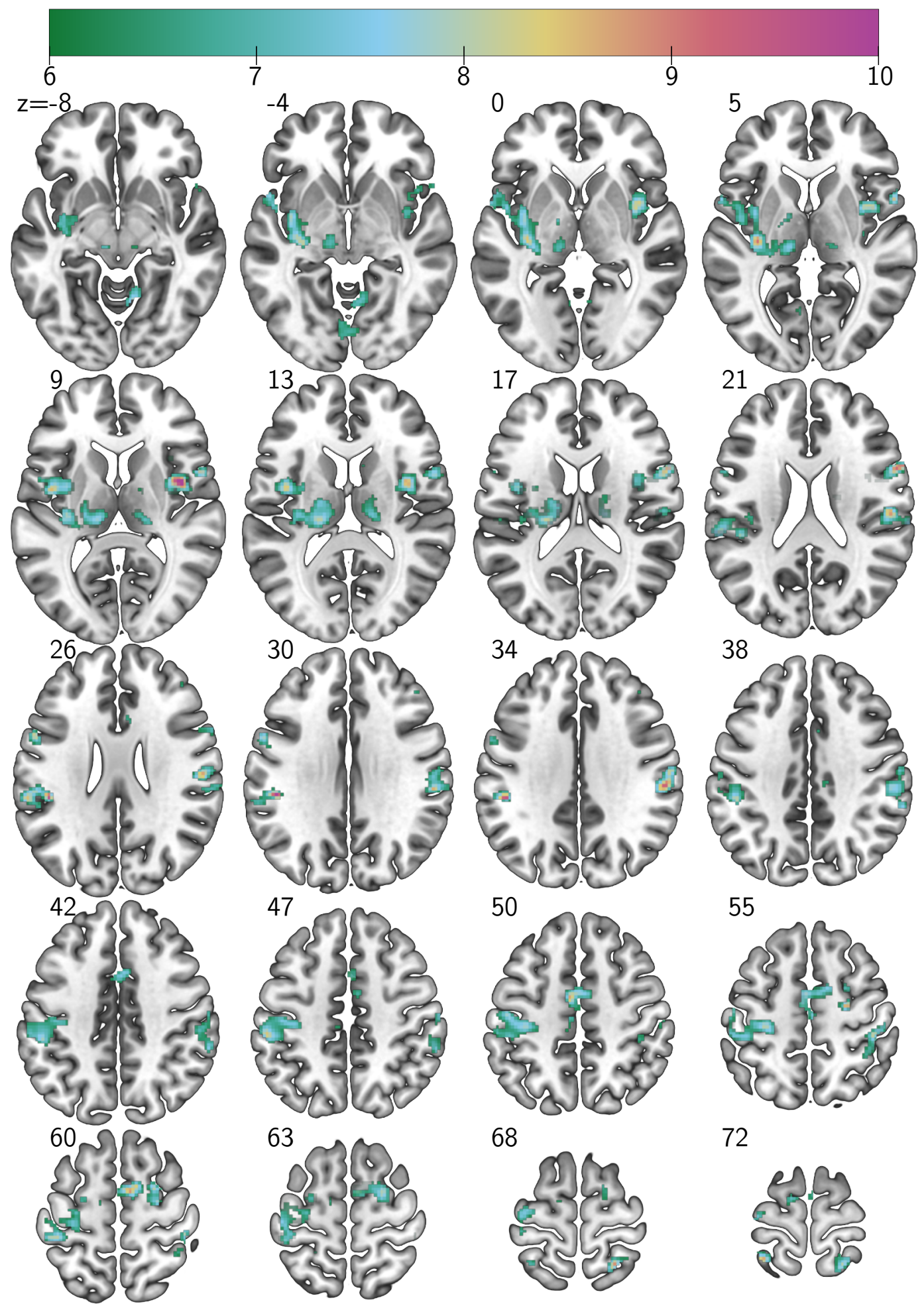

Figure B.1.: Univariate results of mean effect. Location of the significant voxels (thresholded with $\mathrm{p}=0.05$, FWE corrected) within the MNI152 standard brain template. Coloration indicates the negative logarithm of the uncorrected $p$-value of the voxel according to the displayed color bar. Slices use neurological convention (left on the left). $Z$ values of the slices are stated above left of the respective slice. 

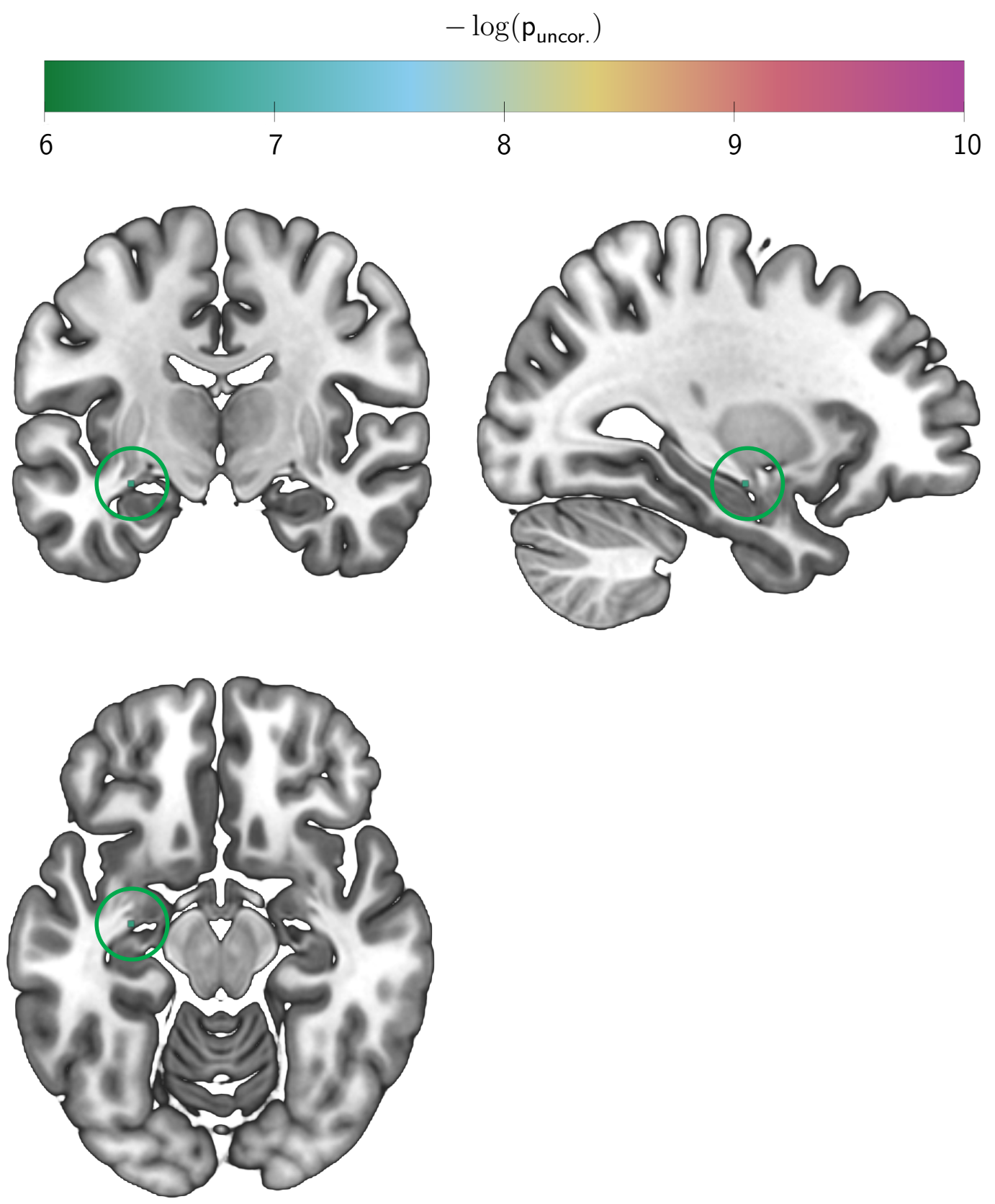

Figure B.2.: Univariate results of reduced main effect predictability. Location of the significant voxel (thresholded with $p=0.05$, FWE corrected) within the MNI152 standard brain template. Coloration indicates the negative logarithm of the uncorrected $\mathrm{p}$-value of the voxel according to the displayed color bar. Axial and coronal slices use neurological convention (left on the left). Circles added for emphasis. 
Table B.2.: Univariate results of reduced main effect predictability. Location of the significant voxel above $\mathrm{p}=0.05$ (FWE corrected). Anatomical labeling as reported by the $\mathrm{AAL}$ atlas.

\begin{tabular}{|c|c|c|c|c|c|}
\hline \multirow[t]{2}{*}{ Anatomical Area } & \multirow[t]{2}{*}{ Size } & \multicolumn{3}{|c|}{ Peak Coordinates } & \multirow{2}{*}{$P_{\text {peak }}$} \\
\hline & & $x$ & Y & Z & \\
\hline hippocampus, (BA 20) & 1 & -30 & -12 & -14 & 0.038 \\
\hline
\end{tabular}

Interaction grasp type $\times$ orientation

This contrast showed no significant voxels.

Reduced interaction grasp orientation $\times$ predictability

This contrast showed no significant voxels.

\section{Reduced interaction grasp type $\times$ predictability}

This contrast showed no significant voxels.

\section{Reduced interaction grasp type $\times$ orientation $\times$ predictability}

This contrast showed no significant voxels.

Main effect visual vs. auditory

See figure B.3 and table B.3 on the following page and on the current page.

Table B.3.: Univariate results of main effect visual vs. auditory. Location of the lowest p-value (voxel wise FWE corrected) and size of surrounding cluster in voxels. Anatomical labelling as reported by the AAL atlas. The labels are sorted by proportion within the cluster, with the label of the peak location in italics.

\begin{tabular}{|c|c|c|c|c|c|}
\hline \multirow{2}{*}{ Anatomical Area } & \multirow[t]{2}{*}{ Size } & \multicolumn{3}{|c|}{ Peak Coordinates } & \multirow[t]{2}{*}{$\mathrm{p}_{\text {peak }}$} \\
\hline & & X & Y & Z & \\
\hline middle occipital gurys, $(\mathrm{BA} 19,18)$ & 8 & 42 & -88 & 0 & 0.011 \\
\hline
\end{tabular}



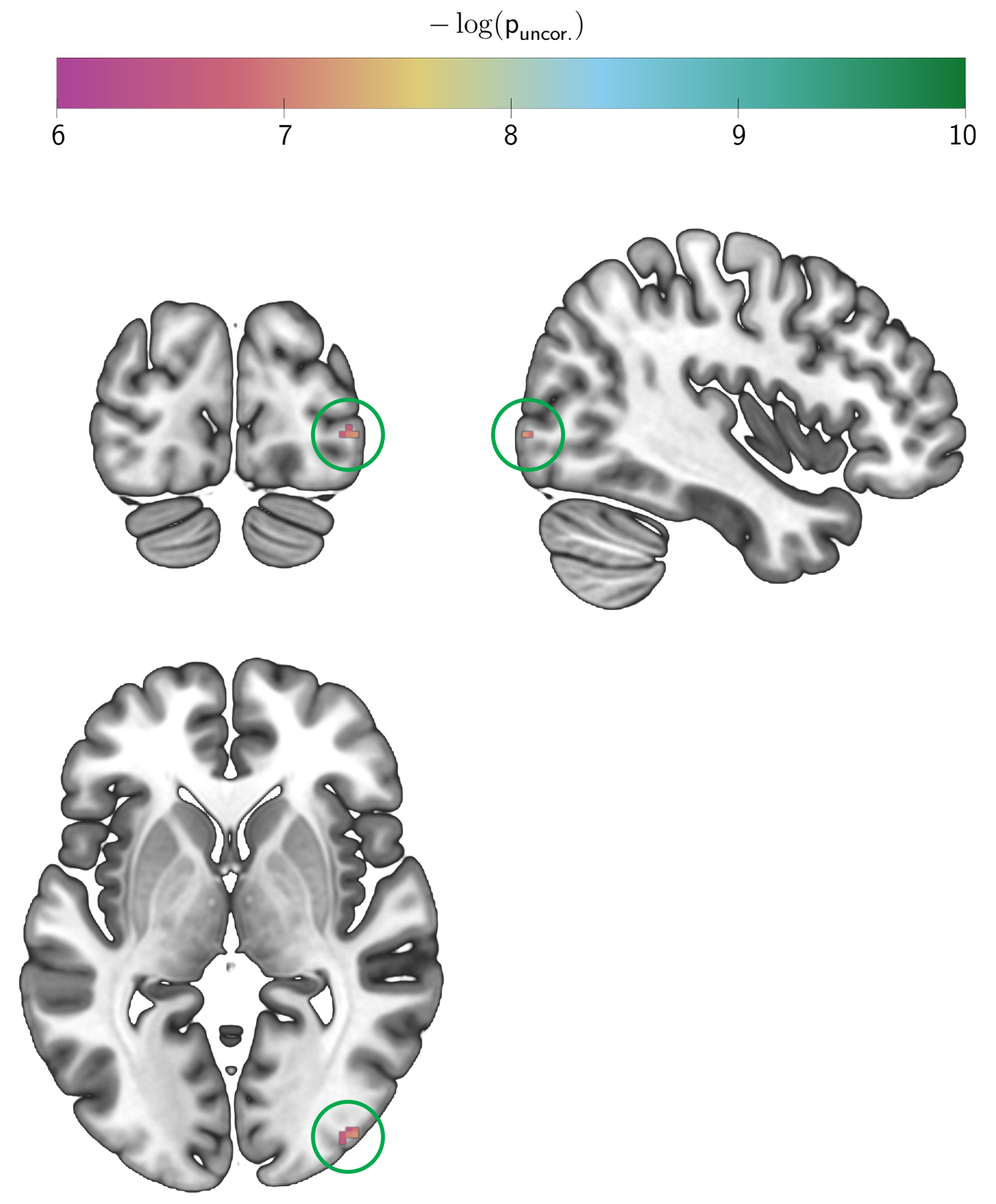

Figure B.3.: Univariate results of main effect visual vs. auditory. Location of the significant voxel (thresholded with $p=0.05$, FWE corrected) within the MNI152 standard brain template. Coloration indicates the negative logarithm of the uncorrected p-value of the voxel according to the displayed color bar. Axial and coronal slices use neurological convention (left on the left). Circles added for emphasis. 


\section{Supplemental Material for project II}




\title{
PriMa: A low-cost, modular, open hardware, and 3D-printed fMRI manipulandum Supplementary Material
}

\author{
R. Stefan Greulich ${ }^{1,2,}$, Timo Hüser ${ }^{1,3,}{ }^{*}$, Matthias Dörge ${ }^{1}$, \\ Hansjörg Scherberger ${ }^{1,2}$ \\ $30^{\text {th }}$ April 2021
}

\author{
Corresponding Author: Hansjörg Scherberger \\ Affiliations: \\ ${ }^{1}$ Deutsches Primatenzentrum GmbH, Kellnerweg 4, 37077 Göttingen, Germany \\ ${ }^{2}$ Faculty of Biology and Psychology, University of Goettingen, Germany \\ ${ }^{3}$ Faculty of Physics, University of Göttingen, Germany \\ * Authors contributed equally to this work
}

\section{S1 Button stress test}

To asses the resilience and service interval of the buttons, we stress tested one button. A servo motor performed a set number of actuations of the button and the required actuation force was measured in regular intervals by putting small weights onto the button until it was fully actuated. As can be seen in Figure 1, the weight necessary for actuation initially decreased rapidly from $0.47 \mathrm{~N}$ to $0.41 \mathrm{~N}$, i.e., by $13 \%$, after the first 200 actuations, but it remained relatively constant afterwards. 


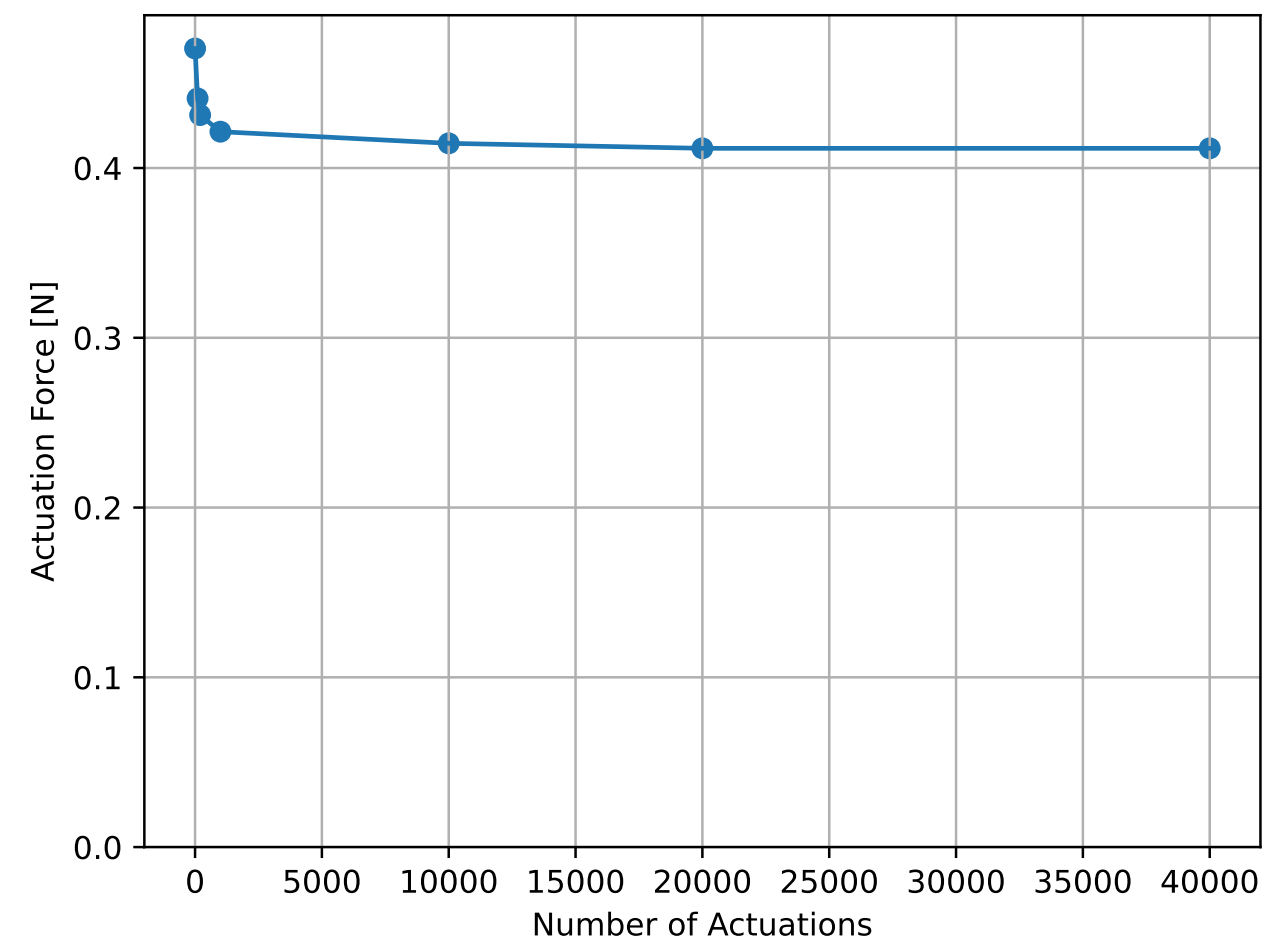

Figure S1: Development of the required actuation force for the precision grip button after given number of actuations.

We therefore recommend to make sure the button was acutated at least 200 times before it is used in any applications that require precise and constant actuation forces. 


\begin{tabular}{ll}
\hline Number of actuations & Actuation force \\
\hline 1 & $0.47 \mathrm{~N}(48 \mathrm{~g})$ \\
100 & $0.44 \mathrm{~N}(45 \mathrm{~g})$ \\
200 & $0.43 \mathrm{~N}(44 \mathrm{~g})$ \\
1000 & $0.42 \mathrm{~N}(43 \mathrm{~g})$ \\
10000 & $0.41 \mathrm{~N}(42.5 \mathrm{~g})$ \\
20000 & $0.41 \mathrm{~N}(42 \mathrm{~g})$ \\
40000 & $0.41 \mathrm{~N}(42 \mathrm{~g})$ \\
\hline
\end{tabular}

Table S1: Development of the required actuation force (in Newton and gram weight) for the precision grip button after given number of actuations.

\section{S2 Additional Pictures human manipulandum}

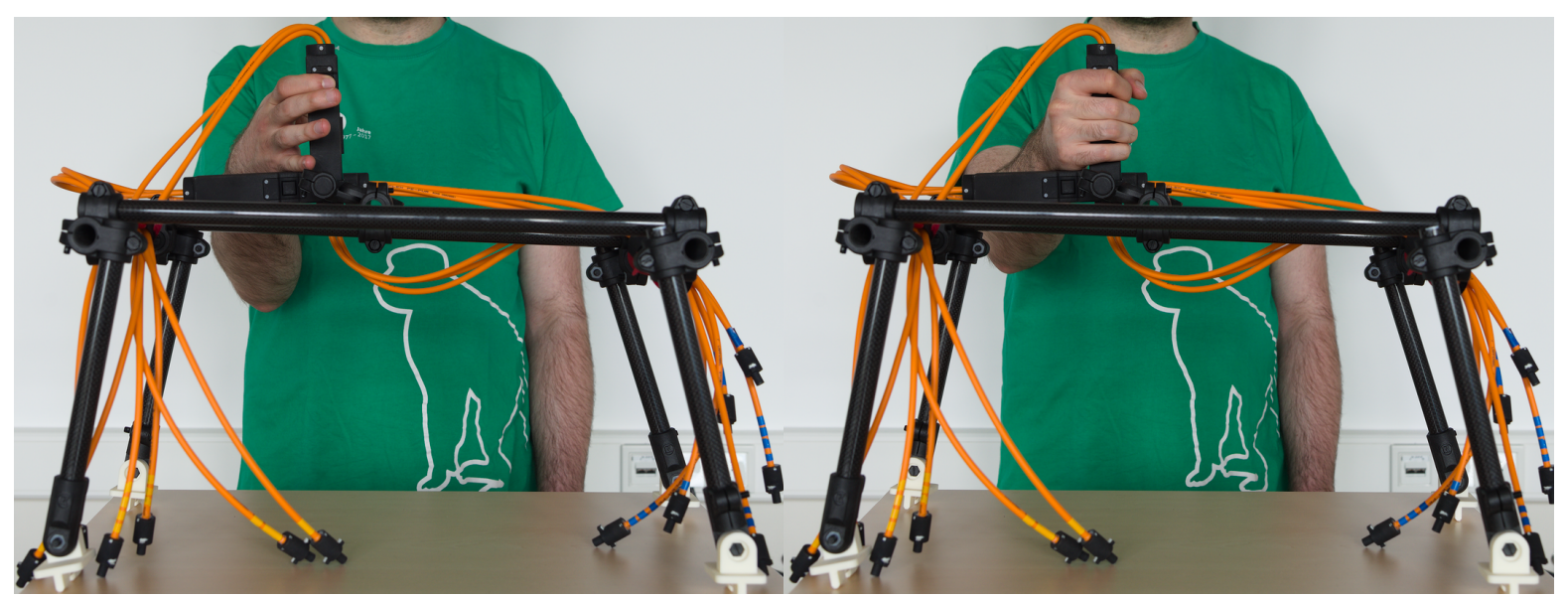

Figure S2: Demonstration of the human manipulandum for the precision (left frame) and power grasp (right frame). Illustrating the entire manipulandum and the mounting frame. 


\section{Additional Resources}

In addition to the cited literature, the following resources were used in the making of this thesis:

Literature research:

Google Scholar

https://scholar.google.com/

Neurosynth

https://www.neurosynth.org/

Image editing:

Gimp Ver. 2.10.12

Inkscape Ver. 1.0beta2 (2b71d25, 2019-12-03)

Adobe Illustrator CS6 Ver. 16.0.0

https://www.gimp.org/

https://inkscape.org/

https://www. adobe.com/de/ products/illustrator.html

3D CAD:

Autodesk Fusion 360

https://www. autodesk.com/ products/fusion-360/overview

English proofreading provided by:

Mentorium $\mathrm{GmbH}$

https://www .mentorium.de/

Typesetting:

LTEX TexLive Ver. 2019.1

https://www . tug.org/texlive/ 


\section{E. Selbstständigkeitserklärung}

Hiermit erkläre ich, dass ich diese Dissertation eigenständig und nur unter Zuhilfenahme der angegebenen Literatur und Hilfsmittel verfasst habe.

Göttingen, den 28.01.2021

R. Stefan Greulich 